\title{
Supporting Information \\ Ambient Decarboxylative Arylation of Malonate Half-Esters via Oxidative Catalysis
}

Patrick J. Moon, Shengkang Yin, Rylan J. Lundgren*

Department of Chemistry, University of Alberta, Edmonton, Alberta, T6G 2G2, Canada rylan.lundgren@ualberta.ca

\section{Contents}

I. General Considerations $\quad$ S-1

II. Synthesis of Substrates S-2

III. Decarboxylative Arylation of Malonate Half Ester Derivatives S-5

IV. Additional Information, Guidelines and Troubleshooting Tips S-19

V. Arene Borylation/Decarboxylative Coupling Sequences S-21

VI. Applications in Complex Molecule Synthesis and Diversification S-24

VII. References S-28

VIII. NMR Spectra S-29

\section{General Considerations}

All glassware and vials were oven-dried prior to use. Flash chromatography was performed as described by Still and co-worker1 (SiliaFlash P60, 40-63 $\mu \mathrm{m}$, 60A silica gel, Silicycle) or by automated flash chromatography (Isolera, HP-SIL or Ultra SNAP silica cartridges, Biotage). Analytical thin-layer chromatography was performed using glass plates pre-coated with silica (SiliaPlate G TLC - Glass-Backed, $250 \mu \mathrm{m}$, Silicycle). TLC plates were visualized by UV light and/or staining with aqueous basic potassium permanganate. Unless otherwise noted, all reagents were obtained from commercial vendors and used as supplied. Boronic esters leading to $\mathbf{2 a - v}, \mathbf{2 z}, \mathbf{4 e}, \mathbf{6 a}, \mathbf{6} \mathbf{b}^{2}$ and $\mathbf{2 w}-\mathbf{y}, 3 \mathbf{a}-\mathbf{i}^{3}$ were synthesized according to the literature procedure from the corresponding boronic acid. Indometacin ethyl ester was synthesized according to the literature procedure from indometacin. ${ }^{4}$ Benzyl malonate halfester was synthesized according to the literature procedure from malonic acid. ${ }^{5}$ Geranyl malonate half-ester was synthesized according to the literature procedure from Meldrum's acid. ${ }^{6}$ Malonyl mono-amide leading to $4 \mathrm{f}$ was synthesized according to the literature procedure from malonic acid. ${ }^{7}$ Select ${ }^{13} \mathrm{C}$ NMR spectra display an artifact signal at 189 or 206 ppm. 


\section{Synthesis of Substrates}

$\mathrm{C}_{\mathrm{O}}^{\mathrm{C}} \mathrm{ll}_{\mathrm{OH}}$

Synthesized according to the literature procedure from Meldrum's acid and the corresponding alcohol in $54 \%$ yield $(1.20 \mathrm{~g}, 5.40 \mathrm{mmol})$ as a light yellow oil. ${ }^{8}$

${ }^{1} \mathrm{H}$ NMR $\left(\mathrm{CDCl}_{3}, 700 \mathrm{MHz}\right) \delta 4.18(\mathrm{~m}, 2 \mathrm{H}), 3.53(\mathrm{~m}, 2 \mathrm{H}), 3.44(\mathrm{~s}, 2 \mathrm{H}), 1.78(\mathrm{~m}, 2 \mathrm{H}), 1.68(\mathrm{~m}$, $2 \mathrm{H}), 1.47(\mathrm{~m}, 2 \mathrm{H}), 1.39(\mathrm{~m}, 2 \mathrm{H})$;

${ }^{13} \mathrm{C}$ NMR $\left(\mathrm{CDCl}_{3}, 176 \mathrm{MHz}\right) \delta 171.6,167.0,66.0,45.0,40.9,32.5,28.4,26.5,25.2$;

HRMS (LCMS ESI): calcd for $\mathrm{C}_{9} \mathrm{H}_{14} \mathrm{ClO}_{4}[\mathrm{M}-\mathrm{H}]=: 221.0586$. Found 221.0590.

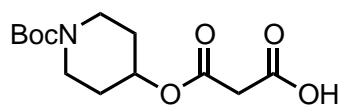

Synthesized according to the literature procedure from Meldrum's acid (1.08 g, $7.5 \mathrm{mmol})$ and corresponding alcohol $(1.39 \mathrm{~g}, 7.5 \mathrm{mmol}) .{ }^{8}$ Isolated in $40 \%$ yield as an off-white solid.

${ }^{1} \mathrm{H}$ NMR $\left(\mathrm{CDCl}_{3}, 700 \mathrm{MHz}\right) \delta 5.27(\mathrm{~m}, 1 \mathrm{H}), 3.67$ (br, 2H), $3.30(\mathrm{~s}, 2 \mathrm{H}) 3.27(\mathrm{~m}, 2 \mathrm{H}), 1.86(\mathrm{br}$, 2H), 1.67 (br, 2H) 1.46 (s, 9H);

${ }^{13} \mathrm{C}$ NMR ( $\left.\mathrm{CDCl}_{3}, 176 \mathrm{MHz}\right)$ 8169.8, 166.6, 80.2, 71.6, 71.6, 41.0, 40.7 (br), 30.4, 28.6;

HRMS (LCMS ESI): calcd for $\mathrm{C}_{13} \mathrm{H}_{20} \mathrm{NO}_{6}$ [M-H]: 286.1296. Found 286.1295.

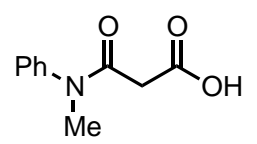

Synthesized according to the literature procedure ${ }^{9}$ using ethyl 3-(methyl(phenyl)amino)-3oxopropanoate (3.32 g, $15.0 \mathrm{mmol}, 1.0$ equiv.) and $\mathrm{LiOH} \bullet \mathrm{H}_{2} \mathrm{O}(629 \mathrm{mg}, 15.0 \mathrm{mmol}, 1.0$ equiv.). Isolated in $95 \%$ yield as a white solid.

${ }^{1} \mathrm{H}$ NMR $\left(\mathrm{CDCl}_{3}, 500 \mathrm{MHz}\right)$ d 7.51-7.47 (m, 2H), $7.43(\mathrm{~m}, 1 \mathrm{H}), 7.22-7.19(\mathrm{~m}, 2 \mathrm{H}), 3.35(\mathrm{~s}$, $3 \mathrm{H}), 3.15(\mathrm{~s}, 2 \mathrm{H})$;

${ }^{13} \mathrm{C}$ NMR $\left(\mathrm{CDCl}_{3}, 125 \mathrm{MHz}\right) \delta$ 169.7, 167.9, 141.5, 130.5, 129.1, 126.8, 37.6, 37.1;

HRMS (LCMS ESI): calcd for $\mathrm{C}_{10} \mathrm{H}_{10} \mathrm{NO}_{3}[\mathrm{M}-\mathrm{H}]=:$ 192.0666. Found 192.0667.

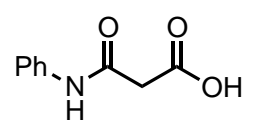

Synthesized according to the literature procedure ${ }^{9}$ using ethyl 3-oxo-3(phenylamino)propanoate ( $3.11 \mathrm{~g}, 15.0 \mathrm{mmol}, 1.0$ equiv.) and $\mathrm{LiOH} \cdot \mathrm{H}_{2} \mathrm{O}(629 \mathrm{mg}, 15.0 \mathrm{mmol}$, 1.0 equiv.). Isolated in $64 \%$ yield as a white solid.

${ }^{1} \mathrm{H}$ NMR (DMSO-d6, $\left.500 \mathrm{MHz}\right) \delta 10.09$ (s, 1H), 7.57 - $7.55(\mathrm{~m}, 2 \mathrm{H}), 7.31$ - $7.28(\mathrm{~m}, 2 \mathrm{H}), 7.04$ $(\mathrm{m}, 1 \mathrm{H}), 3.34(\mathrm{~s}, 2 \mathrm{H})$;

${ }^{13}$ C NMR (DMSO-d6, $125 \mathrm{MHz}$ ) $\delta$ 169.2, 164.5, 138.9, 128.7, 123.4, 119.0, 43.9;

HRMS (LCMS ESI): calcd for $\mathrm{C}_{8} \mathrm{H}_{18} \mathrm{NO}_{3}[\mathrm{M}-\mathrm{H}]=:$ 178.0510. Found 178.0507. 


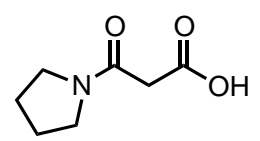

Synthesized according to the literature procedure ${ }^{10}$ using benzyl 3-oxo-3-(pyrrolidin-1yl)propanoate ( $3.21 \mathrm{~g}, 13.0 \mathrm{mmol}, 1.0$ equiv.) and $1 \mathrm{M}$ aq. $\mathrm{NaOH}(22 \mathrm{~mL}, 22 \mathrm{mmol}, 1.7$ equiv.). Isolated in $89 \%$ yield as a white solid.

${ }^{1} \mathrm{H}$ NMR $\left(\mathrm{CDCl}_{3}, 500 \mathrm{MHz}\right) \delta 3.55(\mathrm{t}, J=7.1 \mathrm{~Hz}, 2 \mathrm{H}), 3.45(\mathrm{t}, J=6.9 \mathrm{~Hz}, 2 \mathrm{H}), 3.32(\mathrm{~s}, 2 \mathrm{H})$, $2.07-2.01(\mathrm{~m}, 2 \mathrm{H}), 1.97-1.91(\mathrm{~m}, 2 \mathrm{H})$;

${ }^{13} \mathrm{C}$ NMR $\left(\mathrm{CDCl}_{3}, 125 \mathrm{MHz}\right) \delta$ 168.3, 167.6, 46.7, 46.4, 36.0, 25.7, 24.2;

HRMS (LCMS ESI): calcd for $\mathrm{C}_{7} \mathrm{H}_{10} \mathrm{NO}_{3}[\mathrm{M}-\mathrm{H}]::$ 156.0666. Found 156.0666.

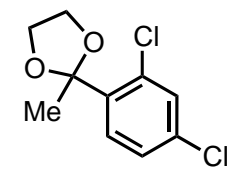

Synthesized according to the literature procedure ${ }^{11}$ using 1-(2,4-dichlorophenyl)ethanone ( $2.00 \mathrm{~g}, 10.6 \mathrm{mmol}, 1.00$ equiv.) and ethylene glycol ( $2.63 \mathrm{~g}, 42.3 \mathrm{mmol}, 4.0$ equiv.). Isolated in $90 \%$ yield as a colorless oil.

${ }^{1} \mathrm{H}$ NMR $\left(\mathrm{CDCl}_{3}, 500 \mathrm{MHz}\right) \delta 7.57(\mathrm{~d}, J=8.3 \mathrm{~Hz}, 1 \mathrm{H}), 7.39(\mathrm{~d}, J=2.1 \mathrm{~Hz}, 1 \mathrm{H}), 7.21(\mathrm{dd}, J=$ 8.5, 2.2 Hz, 1H), $4.10-4.03(\mathrm{~m}, 2 \mathrm{H}), 3.80-3.73(\mathrm{~m}, 2 \mathrm{H}), 1.77$ (s, 3H);

${ }^{13} \mathrm{C}$ NMR $\left(\mathrm{CDCl}_{3}, 125 \mathrm{MHz}\right) \delta$ 138.4, 134.4, 132.8, 131.1, 128.7, 126.7, 108.2, 64.5, 25.2;

HRMS (LCMS EI): calcd for $\mathrm{C}_{9} \mathrm{H}_{7} \mathrm{O}_{2} \mathrm{Cl}_{2}\left[\mathrm{M}-\mathrm{CH}_{3}\right]^{+}: 216.9823$. Found 216.9824. 


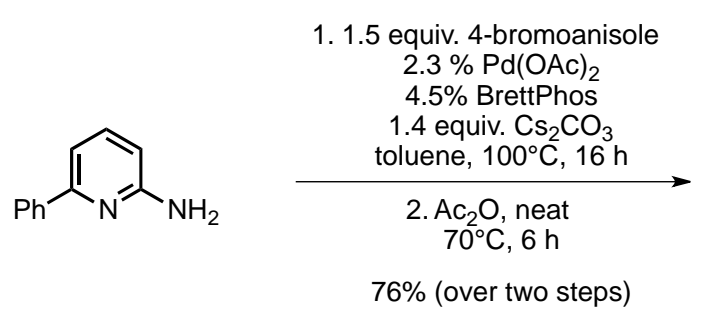

I

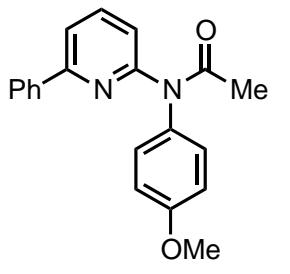

II

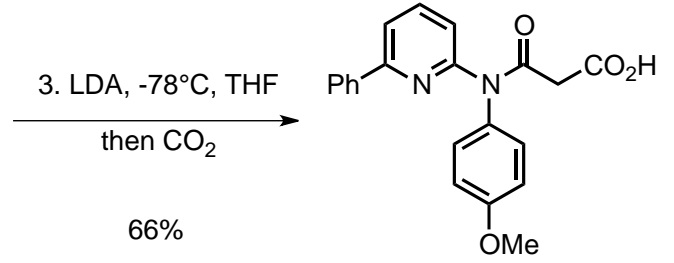

III

Step 1. To a 1-dram vial was added $\mathrm{Pd}(\mathrm{OAc})_{2}(20 \mathrm{mg}, 0.090 \mathrm{mmol}, 0.023$ equiv.) and BrettPhos (97 mg, $0.18 \mathrm{mmol}, 0.045$ equiv.) and toluene $(2.0 \mathrm{~mL})$. This solution was stirred for approximately 5 minutes at room temperature. To an 8 dram vial was added aminopyridine I (681 mg, $4.00 \mathrm{mmol}, 1.00$ equiv.), 4-bromoanisole (0.75 mL, $6.0 \mathrm{mmol}, 1.5$ equiv.), and $\mathrm{Cs}_{2} \mathrm{CO}_{3}$ (1.83 g, $5.60 \mathrm{mmol}, 1.40$ equiv.). The headspace was purged with $\mathrm{N}_{2}$ for 5 minutes, then sealed with a PTFE lined cap. The Pd/Brettphos solution was quantitatively transferred using additional toluene $(18 \mathrm{~mL})$, then the mixture was heated at $100^{\circ} \mathrm{C}$. After $24 \mathrm{~h}$, the reaction was cooled to room temperature, passed through a celite plug, then concentrated in vacuo. The corresponding diarylamine was isolated in $83 \%$ yield after purification by column chromatography (20:1 to 7:3 Hexane/EtOAc).

Step 2. To a vial containing the diarylamine $(848 \mathrm{mg}, 3.10 \mathrm{mmol}, 1.00$ equiv.) was added acetic anhydride $\left(1.5 \mathrm{~mL}, 15 \mathrm{mmol}, 5.0\right.$ equiv.), then heated at $70^{\circ} \mathrm{C}$. After $6 \mathrm{~h}$, the reaction was cooled to room temperature and concentrated in vacuo. The crude material was dissolved in $30 \mathrm{ml}$ EtOAc, then washed sequentially with sat. aq. $\mathrm{NaHCO}_{3}(3 \times 10 \mathrm{~mL})$, then brine $(10$ $\mathrm{mL}$ ). The organic portion was dried with $\mathrm{Na}_{2} \mathrm{SO}_{4}$, then concentrated in vacuo to afford the acetate II in 91\% yield as a colorless oil.

${ }^{1}$ H NMR $\left(\mathrm{CDCl}_{3}, 500 \mathrm{MHz}\right) \delta 7.91-7.89(\mathrm{~m}, 2 \mathrm{H}), 7.74(\mathrm{~m}, 1 \mathrm{H}), 7.57(\mathrm{~m}, 1 \mathrm{H}), 7.44-7.34(\mathrm{~m}$, $4 \mathrm{H}), 7.31-7.27(\mathrm{~m}, 2 \mathrm{H}), 6.96-6.93(\mathrm{~m}, 2 \mathrm{H}), 3.83(\mathrm{~s}, 3 \mathrm{H}), 2.19(\mathrm{~s}, 3 \mathrm{H})$;

${ }^{13} \mathrm{C} \mathrm{NMR}\left(\mathrm{CDCl}_{3}, 125 \mathrm{MHz}\right) \delta$ 171.5, 158.8, 156.1, 155.1, 138.6, 138.5, 135.0, 129.8, 129.2, 128.7, 126.8, 118.8, 117.3, 114.6, 55.5, 24.6;

HRMS (LCMS ESI): calcd for $\mathrm{C}_{20} \mathrm{H}_{18} \mathrm{~N}_{2} \mathrm{NaO}_{2}[\mathrm{M}+\mathrm{Na}]^{+}: 341.1260$. Found 341.1268.

Step 3. To a solution of LDA (3.1 mmol, 1.1 equiv.) in THF $(0.3 \mathrm{M})$ at $-78^{\circ} \mathrm{C}$ was added II (897 mg, $2.82 \mathrm{mmol}, 1.0$ equiv.) as a solution in THF (7 mL), to give a yellow solution. After 30 minutes, $\mathrm{CO}_{2}$ was bubbled into the solution. After 20 minutes, the reaction was slowly warmed to room temperature and stirred for 30 minutes. Excess $\mathrm{CO}_{2}$ was added and stirred for an additional 30 minutes. The reaction was quenched with water $(30 \mathrm{~mL})$ washed with $\mathrm{Et}_{2} \mathrm{O}(3 \times 20 \mathrm{~mL})$. The aqueous layer was acidified to $\mathrm{pH} 3$ and extracted with EtOAc $(3 \times 15$ $\mathrm{mL}$ ). The combined organic layers were dried with $\mathrm{Na}_{2} \mathrm{SO}_{4}$ and concentrated in vacuo to afford III in $66 \%$ yield as an off-white solid (85\% purity). This material slowly decarboxylates at room temperature and should be used immediately or stored in a freezer. 


\section{Decarboxylative Arylation of Malonate Half Ester Derivatives}

General Procedure A: $\mathrm{Cu}(\mathrm{OTf})_{2}(54.3 \mathrm{mg}, 0.150 \mathrm{mmol}, 0.30$ equiv.) and arylboronic ester $(1.00$ mmol, 1.20 to 2.00 equiv.) were added sequentially to a 1 dram vial charged with a stirbar. The carboxylic acid $(0.500 \mathrm{mmol}, 1.00$ equiv.) was added as a solution in anhydrous DMA (0.6 $\mathrm{mL})$. Additional DMA ( $2 \times 0.3 \mathrm{~mL})$ was used to quantitatively transfer the solution to the reaction mixture. The solution was stirred until a homogeneous pale blue solution was formed (approximately 2 minutes, partially heterogeneous mixtures obtained when using increased $\mathrm{Cu}$ loadings), followed by the addition of triethylamine $(0.42 \mathrm{~mL}, 3.0 \mathrm{mmol}, 6.0$ equiv.). The vial was sealed with a PTFE-lined cap, exposed to air via a needle, and gently stirred at room temperature. Upon completion of the reaction ( 24 to $72 \mathrm{~h}$ ), the reaction mixture was diluted with EtOAc $(40 \mathrm{~mL})$, and washed sequentially with $\mathrm{NH}_{4} \mathrm{Cl}(15 \mathrm{~mL}), 0.5$ $\mathrm{M} \mathrm{NaOH}(2 \times 20 \mathrm{~mL})$, and brine $(15 \mathrm{~mL})$. The organic layer was dried with $\mathrm{Na}_{2} \mathrm{SO}_{4}$, concentrated in vacuo, and purified by silica gel chromatography. No difference was observed if reactions were prepared in an atmosphere-controlled glovebox, then exposed to ambient air.

Procedure for Gram Scale Reaction: To a $50 \mathrm{~mL}$ pear-shaped round bottomed flask in air was added $\mathrm{Cu}(\mathrm{OTf})_{2}$ (942 mg, $2.16 \mathrm{mmol}, 0.30$ equiv.), 3-iodophenyl neopentyl boronic ester (4.55 g, $14.4 \mathrm{mmol}, 2.0$ equiv.), DMA (18 mL) and mono-ethyl malonate $(951 \mathrm{mg}, 7.2 \mathrm{mmol}, 1.0$ equiv.). The mixture was stirred for 10 minutes to generate a suspension to which NEt3 $(6.0$ $\mathrm{mL}, 43 \mathrm{mmol}, 6$ equiv.) was added. After 24 hours the reaction was diluted with saturated aqueous $\mathrm{NH}_{4} \mathrm{Cl}$ and $\mathrm{EtOAc}$, the organic layer was extracted, washed with aqueous $\mathrm{KOH}$ and brine, dried with $\mathrm{Na}_{2} \mathrm{SO}_{4}$ and concentrated in vacuo and purified by silica gel chromatography (Hexane/EtOAc gradient). The product 2a was obtained in 76\% (run 1) and 78\% (run 2) yield.

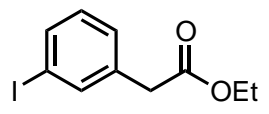

2a Prepared according to the General Procedure A from the corresponding neopentyl boronic ester (316 mg, $1.00 \mathrm{mmol}, 2.00$ equiv.) and mono-ethyl malonate (66.1 mg, 0.500 mmol, 1.00 equiv.) using $\mathrm{Cu}(\mathrm{OTf})_{2}(54.3 \mathrm{mg}, 0.150 \mathrm{mmol}, 0.300$ equiv.), $71 \mathrm{~h}$. Isolated in $83 \%$ yield after purification by column chromatography (15:1 Hexane/EtOAc) as a colorless oil.

${ }^{1} \mathrm{H} \mathrm{NMR}\left(\mathrm{CDCl}_{3}, 500 \mathrm{MHz}\right) \delta 7.65(\mathrm{~m}, 1 \mathrm{H}), 7.61(\mathrm{~m}, 1 \mathrm{H}), 7.26(\mathrm{~m}, 1 \mathrm{H}), 7.06(\mathrm{~m}, 1 \mathrm{H}), 4.16(\mathrm{q}$, $J=7.0 \mathrm{~Hz}, 2 \mathrm{H}), 3.55(\mathrm{~s}, 2 \mathrm{H}), 1.26(\mathrm{t}, J=7.0 \mathrm{~Hz}, 3 \mathrm{H})$;

${ }^{13} \mathrm{C}$ NMR $\left(\mathrm{CDCl}_{3}, 125 \mathrm{MHz}\right) \delta 171.1,138.4,136.5,136.3,130.3,128.7,94.5,61.2,40.9,14.3$;

HRMS (LCMS ESI): calcd for $\mathrm{C}_{10} \mathrm{H}_{11} \mathrm{INaO}_{2}[\mathrm{M}+\mathrm{Na}]^{+}: 312.9696$. Found 312.9696.

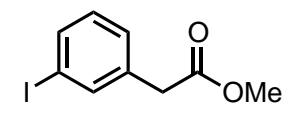

2b Prepared according to the General Procedure from the corresponding neopentyl boronic ester ( $316 \mathrm{mg}, 1.00 \mathrm{mmol}, 2.00$ equiv.) and mono-methyl malonate (59.0 mg, 0.500 mmol, 1.00 equiv.) using $\mathrm{Cu}(\mathrm{OTf}) 2(54.3 \mathrm{mg}, 0.150 \mathrm{mmol}, 0.300$ equiv.), $66 \mathrm{~h}$. Isolated in $78 \%$ 
yield after purification by column chromatography (Hexane/EtOAc gradient) as a light yellow oil.

${ }^{1} \mathrm{H}$ NMR $\left(\mathrm{CDCl}_{3}, 700 \mathrm{MHz}\right) \delta 7.65(\mathrm{~m} \mathrm{1H}), 7.61(\mathrm{~m}, 1 \mathrm{H}), 7.25(\mathrm{~m}, 1 \mathrm{H}) 7.06(\mathrm{t}, J=6.3 \mathrm{~Hz}, 1 \mathrm{H})$, $3.70(\mathrm{~s} 3 \mathrm{H}), 3.57$ (s, 2H);

${ }^{13} \mathrm{C}$ NMR $\left(\mathrm{CDCl}_{3}, 176 \mathrm{MHz}\right) \delta 171.5,138.4,136.4,136.3,130.4,128.7,94.5,52.3,40.7$;

HRMS (EI): calcd for $\mathrm{C}_{9} \mathrm{H}_{9} \mathrm{IO}_{2} \mathrm{M}^{+}: 275.9647$. Found 275.9649 .

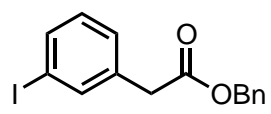

2c Prepared according to the General Procedure A from the corresponding neopentyl boronic ester (316 mg, $1.00 \mathrm{mmol}, 2.00$ equiv.) and mono-benzyl malonate $(97.1 \mathrm{mg}, 0.500$ mmol, 1.00 equiv.) using $\mathrm{Cu}(\mathrm{OTf})_{2}(54.3 \mathrm{mg}, 0.150 \mathrm{mmol}, 0.300$ equiv.), $48 \mathrm{~h}$. Isolated in $66 \%$ yield after purification by column chromatography (20:1 to 10:1 Hexane/EtOAc) as a colorless oil.

${ }^{1} \mathrm{H}$ NMR $\left(\mathrm{CDCl}_{3}, 500 \mathrm{MHz}\right) \delta 7.65(\mathrm{~m}, 1 \mathrm{H}), 7.61(\mathrm{~m}, 1 \mathrm{H}), 7.30-7.38(\mathrm{~m}, 5 \mathrm{H}), 7.25(\mathrm{~m}, 1 \mathrm{H})$, $7.06(\mathrm{~m}, 1 \mathrm{H}), 5.14(\mathrm{~s}, 2 \mathrm{H}), 3.61(\mathrm{~s}, 2 \mathrm{H})$;

${ }^{13} \mathrm{C}$ NMR $\left(\mathrm{CDCl}_{3}, 125 \mathrm{MHz}\right) \delta 170.7,138.3,136.3,136.1,135.7,130.3,128.7,128.6,128.4$, 128.2, 94.4, 66.9, 40.7;

HRMS (LCMS ESI): calcd for $\mathrm{C}_{15} \mathrm{H}_{13} \mathrm{INaO}_{2}[\mathrm{M}+\mathrm{Na}]^{+}: 374.9852$ Found 374.9854 .

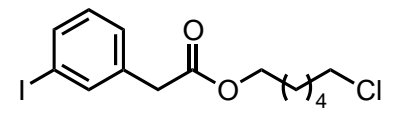

2d Prepared according to the General Procedure A from the corresponding neopentyl boronic ester (316 mg, $1.00 \mathrm{mmol}, 2.00$ equiv.) and mono-1-chlorohexyl malonate (120 mg, $0.500 \mathrm{mmol}, 1.00$ equiv.) using $\mathrm{Cu}(\mathrm{OTf})_{2}(54.3 \mathrm{mg}, 0.150 \mathrm{mmol}, 0.300$ equiv.), $60 \mathrm{~h}$. Isolated in $93 \%$ yield after purification by column chromatography (40:1 to 5:1 Hexane/EtOAc) as a colorless oil.

${ }^{1} \mathrm{H}$ NMR $\left(\mathrm{CDCl}_{3}, 500 \mathrm{MHz}\right) \delta 7.65(\mathrm{~m}, 1 \mathrm{H}), 7.61(\mathrm{~m}, 1 \mathrm{H}), 7.25(\mathrm{~m}, 1 \mathrm{H}), 7.06(\mathrm{~m}, 1 \mathrm{H}), 4.10(\mathrm{t}$, $\mathrm{J}=6.5 \mathrm{~Hz}, 2 \mathrm{H}), 3.55(\mathrm{~s}, 2 \mathrm{H}), 3.52(\mathrm{t}, \mathrm{J}=6.7 \mathrm{~Hz}, 2 \mathrm{H}), 1.78-1.74(\mathrm{~m}, 2 \mathrm{H}), 1.66-1.61(\mathrm{~m}, 2 \mathrm{H}), 1.46$ $-1.42(\mathrm{~m}, 2 \mathrm{H}), 1.36-1.31(\mathrm{~m}, 2 \mathrm{H})$;

${ }^{13} \mathrm{C}$ NMR $\left(\mathrm{CDCl}_{3}, 175 \mathrm{MHz}\right) \delta$ 171.0, 138.3, 136.4, 136.2, 130.3, 128.6, 94.4, 65.0, 45.0, 40.9, 32.5, 28.4, 26.5, 25.2;

HRMS (LCMS ESI): calcd for $\mathrm{C}_{14} \mathrm{H}_{18} \mathrm{ClINaO}_{2}[\mathrm{M}+\mathrm{Na}]^{+}: 402.9932$. Found 402.9932.

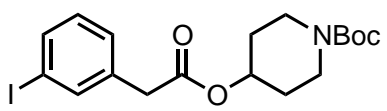

2e Prepared according to the General Procedure from the corresponding neopentyl boronic ester ( $316 \mathrm{mg}, 1.00 \mathrm{mmol}, 2.00$ equiv.) and mono-4-NBoc piperidyl malonate (143.6 $\mathrm{mg}, 0.500 \mathrm{mmol}, 1.00$ equiv.) using $\mathrm{Cu}(\mathrm{OTf})_{2}(54.3 \mathrm{mg}, 0.150 \mathrm{mmol}, 0.300$ equiv.), $66 \mathrm{~h}$. Isolated in $47 \%$ yield after purification by column chromatography (4:1 Hexane/EtOAc) as a colorless oil. 
${ }^{1} \mathrm{H}$ NMR $\left(\mathrm{CDCl}_{3}, 700 \mathrm{MHz}\right) \delta 7.65(\mathrm{~m} \mathrm{1H}), 7.61(\mathrm{~m}, 1 \mathrm{H}), 7.25(\mathrm{~m}, 1 \mathrm{H}) 7.06(\mathrm{t}, J=7.7 \mathrm{~Hz}, 1 \mathrm{H})$, $4.94(\mathrm{~m}, 1 \mathrm{H}), 3.62(\mathrm{br}, 2 \mathrm{H}), 3.55(\mathrm{~s}, 2 \mathrm{H}), 3.23(\mathrm{~m}, 2 \mathrm{H}), 1.81(\mathrm{br}, 2 \mathrm{H}), 1.58(\mathrm{br}, 2 \mathrm{H}), 1.46(\mathrm{~s}, 9 \mathrm{H})$;

${ }^{13} \mathrm{C}$ NMR $\left(\mathrm{CDCl}_{3}, 176 \mathrm{MHz}\right) \delta 170.3,154.8,138.3,136.4,136.3,130.4,128.6,94.5,79.9,70.6$, 41.2, 41.1(br), 30.6, 28.6;

HRMS (LCMS ESI): calcd for $\mathrm{C}_{18} \mathrm{H}_{24} \mathrm{INNaO}_{4}[\mathrm{M}+\mathrm{Na}]^{+}: 468.0642$. Found 468.0641 .

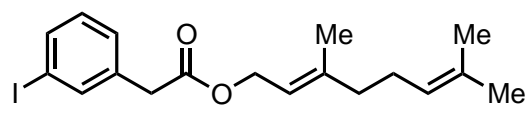

2f Prepared according to the General Procedure from the corresponding neopentyl boronic ester ( $316 \mathrm{mg}, 1.00 \mathrm{mmol}, 2.00$ equiv.) and mono-geranyl malonate (8:1 mixture of $E / Z$ isomers, $120 \mathrm{mg}$, $0.500 \mathrm{mmol}, 1.00$ equiv.) using $\mathrm{Cu}(\mathrm{OTf})_{2}(54.3 \mathrm{mg}, 0.150 \mathrm{mmol}, 0.300$ equiv.), $73 \mathrm{~h}$. Isolated in $45 \%$ yield after purification by column chromatography (Hexane/EtOAc gradient) as a colorless oil (mixture of $E / Z$ isomers [8:1] as in the geraniol starting material):

${ }^{1} \mathrm{H}$ NMR $\left(\mathrm{CDCl}_{3}, 700 \mathrm{MHz}\right) \delta 7.65(\mathrm{~m}, 1 \mathrm{H}), 7.60(\mathrm{~m}, 1 \mathrm{H}), 7.25(\mathrm{~d}, J=8.5 \mathrm{~Hz}, 1 \mathrm{H}) 7.05(\mathrm{t}, J=$ $7.8 \mathrm{~Hz}, 1 \mathrm{H}), 5.33(\mathrm{~m}, 1 \mathrm{H}), 5.08(\mathrm{~m}, 1 \mathrm{H}), 4.62(\mathrm{~d}, J=7.0 \mathrm{~Hz}, 2 \mathrm{H}, E$ isomer $), 4.60(\mathrm{~d}, J=7.3 \mathrm{~Hz}$, $2 \mathrm{H}, \mathrm{Z}$ isomer), $3.56(\mathrm{~s}, 2 \mathrm{H}), 2.13-2.07(\mathrm{~m}, 2 \mathrm{H}), 2.07-2.02(\mathrm{~m}, 2 \mathrm{H}), 1.70(\mathrm{~m}, 6 \mathrm{H}), 1.60(\mathrm{~s}, 3 \mathrm{H})$;

${ }^{13} \mathrm{C}$ NMR $\left(\mathrm{CDCl}_{3}, 176 \mathrm{MHz}\right) \delta 170.1,142.9,138.4,136.5,136.3,132.0,130.3,128.7,123.9$, 118.1, 94.5, 62.1, 40.9, 40.0, 26.4, 25.8, 17.9, 16.7;

HRMS (LCMS ESI): calcd for $\mathrm{C}_{18} \mathrm{H}_{23} \mathrm{INaO}_{2}[\mathrm{M}+\mathrm{Na}]^{+}: 421.0635$. Found 421.0635.

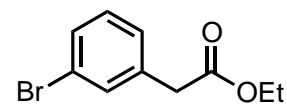

$2 \mathrm{~g}$ Prepared according to the General Procedure A from the corresponding neopentyl boronic ester (268 mg, $1.00 \mathrm{mmol}, 2.00$ equiv.) and mono-ethyl malonate (66.1 mg, 0.500 mmol, 1.00 equiv.) using $\mathrm{Cu}(\mathrm{OTf})_{2}(54.3 \mathrm{mg}, 0.150 \mathrm{mmol}, 0.300$ equiv.), $72 \mathrm{~h}$. Isolated in $82 \%$ yield after purification by column chromatography (15:1 Hexane/EtOAc) as a colorless oil. Spectroscopic data agreed with that reported..$^{12}$<smiles>CCOC(=O)Cc1cccc(Cl)c1</smiles>

2h Prepared according to the General Procedure A from the corresponding neopentyl boronic ester (197 mg, $0.880 \mathrm{mmol}, 2.00$ equiv.) and mono-ethyl malonate (58.1 mg, 0.440 mmol, 1.00 equiv.) using $\mathrm{Cu}(\mathrm{OTf})_{2}(47.7 \mathrm{mg}, 0.132 \mathrm{mmol}, 0.300$ equiv.), $62 \mathrm{~h}$. Isolated in $77 \%$ yield after purification by column chromatography (20:1 to 10:1 Hexane/EtOAc) as a colorless oil. Spectroscopic data agreed with that reported. ${ }^{13}$ 
<smiles>CCOC(=O)Cc1cccc([N+](=O)[O-])c1</smiles>

2i Prepared according to the General Procedure A from the corresponding neopentyl boronic ester ( $235 \mathrm{mg}, 1.00 \mathrm{mmol}, 2.00$ equiv.) and mono-ethyl malonate $(66.1 \mathrm{mg}, 0.500$ mmol, 1.00 equiv.) using $\mathrm{Cu}(\mathrm{OTf})_{2}(54.3 \mathrm{mg}, 0.150 \mathrm{mmol}, 0.300$ equiv.), $67 \mathrm{~h}$. Isolated in $68 \%$ yield after purification by column chromatography (10:1 to 1:1 Hexane/EtOAc) as a colorless oil. Spectroscopic data agreed with that reported. ${ }^{14}$<smiles>CCOC(=O)Cc1cccc(C#N)c1</smiles>

2j Prepared according to the General Procedure A from the corresponding neopentyl boronic ester $(215 \mathrm{mg}, 1.00 \mathrm{mmol}, 2.00$ equiv.) and mono-ethyl malonate $(66.1 \mathrm{mg}, 0.500$ mmol, 1.00 equiv.) using $\mathrm{Cu}(\mathrm{OTf})_{2}(54.3 \mathrm{mg}, 0.150 \mathrm{mmol}, 0.300$ equiv.), $60 \mathrm{~h}$. Isolated in $65 \%$ yield after purification by column chromatography (4:1 Hexane/EtOAc) as a light beige solid. Spectroscopic data agreed with that reported. ${ }^{14}$<smiles>CCOC(=O)Cc1cccc(OC)c1</smiles>

2k Prepared according to the General Procedure A from the corresponding neopentyl boronic ester $(220 \mathrm{mg}, 1.00 \mathrm{mmol}, 2.00$ equiv.) and mono-ethyl malonate (66.1 mg, 0.500 mmol, 1.00 equiv.) using $\mathrm{Cu}(\mathrm{OTf})_{2}(181 \mathrm{mg}, 0.500 \mathrm{mmol}, 1.00$ equiv.), $50 \mathrm{~h}$. Isolated in $67 \%$ yield after purification by column chromatography (50:1 to 4:1 Hexane/EtOAc) as a colorless oil. Spectroscopic data agreed with that reported. ${ }^{14}$

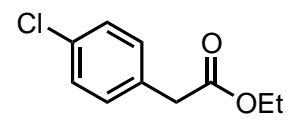

21 Prepared according to the General Procedure A from the corresponding neopentyl boronic ester (225 mg, $1.00 \mathrm{mmol}, 2.00$ equiv.) and mono-ethyl malonate $(66.1 \mathrm{mg}, 0.500$ mmol, 1.00 equiv.) using $\mathrm{Cu}(\mathrm{OTf})_{2}(54.3 \mathrm{mg}, 0.150 \mathrm{mmol}, 0.300$ equiv.), $72 \mathrm{~h}$. Isolated in $68 \%$ yield after purification by column chromatography (15:1 Hexane/EtOAc) as a colorless oil. Spectroscopic data agreed with that reported. ${ }^{13}$<smiles>CCOC(=O)Cc1ccc(C(=O)OCC)cc1</smiles>

$2 \mathrm{~m}$ Prepared according to the General Procedure A from the corresponding neopentyl boronic ester (262 mg, $1.00 \mathrm{mmol}, 2.00$ equiv.) and mono-ethyl malonate (66.1 mg, 0.500 mmol, 1.00 equiv.) using $\mathrm{Cu}(\mathrm{OTf})_{2}(54.3 \mathrm{mg}, 0.150 \mathrm{mmol}, 0.300$ equiv.), $70 \mathrm{~h}$. Isolated in $75 \%$ yield after purification by column chromatography (15:1 Hexane/EtOAc) as a colorless oil. Spectroscopic data agreed with that reported. ${ }^{15}$ 


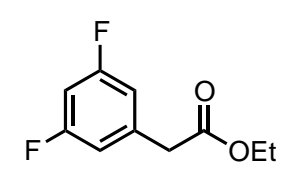

2n Prepared according to the General Procedure A from the corresponding neopentyl boronic ester (226 mg, $1.00 \mathrm{mmol}, 2.00$ equiv.) and mono-ethyl malonate (66.1 mg, 0.500 mmol, 1.00 equiv.) using $\mathrm{Cu}(\mathrm{OTf})_{2}(54.3 \mathrm{mg}, 0.150 \mathrm{mmol}, 0.300$ equiv.), $42 \mathrm{~h}$. Isolated in $77 \%$ yield after purification by column chromatography $(10: 1$ Hexane/EtOAc) as a colorless oil.

${ }^{1} \mathrm{H} \mathrm{NMR}\left(\mathrm{CDCl}_{3}, 500 \mathrm{MHz}\right) \delta 6.84-6.80(\mathrm{~m}, 2 \mathrm{H}), 6.72(\mathrm{~m}, 1 \mathrm{H}), 4.17(\mathrm{q}, J=7.2 \mathrm{~Hz}, 2 \mathrm{H}), 3.59$ $(\mathrm{s}, 2 \mathrm{H}), 1.27(\mathrm{t}, J=7.1 \mathrm{~Hz}, 3 \mathrm{H})$;

${ }^{13} \mathrm{C}$ NMR $\left(\mathrm{CDCl}_{3}, 125 \mathrm{MHz}\right) \delta 170.4,163.0(\mathrm{dd}, J=249,12.9 \mathrm{~Hz}), 137.6,112.4,102.7,61.3$, 41.0, 14.2;

${ }^{19} \mathrm{~F} \mathrm{NMR}\left(\mathrm{CDCl}_{3}, 469 \mathrm{MHz}\right) \delta-110.0(\mathrm{t}, J=8.2 \mathrm{~Hz})$;

HRMS (EI): calcd for $\mathrm{C}_{10} \mathrm{H}_{10} \mathrm{O}_{2} \mathrm{~F}_{2}[\mathrm{M}]^{+}: 200.0649$. Found 200.0647 .

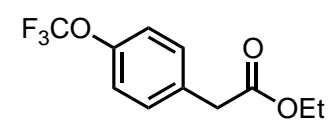

2o Prepared according to the General Procedure A from the corresponding neopentyl boronic ester (274 mg, $1.00 \mathrm{mmol}, 2.00$ equiv.) and mono-ethyl malonate (66.1 mg, 0.500 mmol, 1.00 equiv.) using $\mathrm{Cu}(\mathrm{OTf}) 2(54.3 \mathrm{mg}, 0.150 \mathrm{mmol}, 0.300$ equiv.), $60 \mathrm{~h}$. Isolated in $60 \%$ yield after purification by column chromatography (50:1 to 4:1 Hexane/EtOAc) as a colorless oil. Spectroscopic data agreed with that reported. ${ }^{15}$

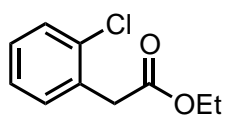

$2 p$ Prepared according to the General Procedure A from the corresponding neopentyl boronic ester (225 mg, $1.00 \mathrm{mmol}, 2.00$ equiv.) and mono-ethyl malonate (66.1 mg, 0.500 mmol, 1.00 equiv.) using $\mathrm{Cu}(\mathrm{OTf})_{2}(54.3 \mathrm{mg}, 0.150 \mathrm{mmol}, 0.300$ equiv.), $42 \mathrm{~h}$. Isolated in $47 \%$ yield after purification by column chromatography (10:1 Hexane/EtOAc) as a colorless oil. Spectroscopic data agreed with that reported. ${ }^{13}$

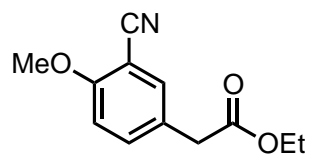

2q Prepared according to the General Procedure A from the corresponding neopentyl boronic ester ( $245 \mathrm{mg}, 1.00 \mathrm{mmol}, 2.00$ equiv.) and mono-ethyl malonate (66.1 mg, 0.500 mmol, 1.00 equiv.) using $\mathrm{Cu}(\mathrm{OTf}) 2(54.3 \mathrm{mg}, 0.150 \mathrm{mmol}, 0.300$ equiv.), $62 \mathrm{~h}$. Isolated in $69 \%$ yield after purification by column chromatography (2:1 Hexane/EtOAc) as a white solid.

${ }^{1} \mathrm{H}$ NMR $\left(\mathrm{CDCl}_{3}, 500 \mathrm{MHz}\right) \delta 7.46(\mathrm{~m}, 2 \mathrm{H}), 6.92(\mathrm{~m}, 1 \mathrm{H}), 4.15(\mathrm{q}, J=7.1 \mathrm{~Hz}, 2 \mathrm{H}), 3.92(\mathrm{~s}$, $3 \mathrm{H}), 3.55(\mathrm{~s}, 2 \mathrm{H}), 1.26(\mathrm{t}, J=7.2 \mathrm{~Hz}, 3 \mathrm{H})$;

${ }^{13} \mathrm{C}$ NMR $\left(\mathrm{CDCl}_{3}, 125 \mathrm{MHz}\right) \delta$ 170.9, 160.4, 135.4, 134.4, 126.8, 116.3, 111.5, 102.0, 61.2, 56.2, $39.9,14.2$; 
HRMS (LCMS ESI): calcd for $\mathrm{C}_{12} \mathrm{H}_{13} \mathrm{NNaO}_{3}[\mathrm{M}+\mathrm{Na}]^{+}:$242.0788. Found 242.0789.

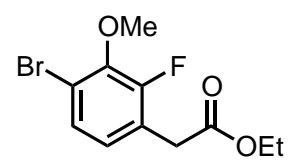

2r Prepared according to the General Procedure A from the corresponding neopentyl boronic ester ( $317 \mathrm{mg}, 1.00 \mathrm{mmol}, 2.00$ equiv.) and mono-ethyl malonate (66.1 mg, 0.500 mmol, 1.00 equiv.) using $\mathrm{Cu}(\mathrm{OTf})_{2}(54.3 \mathrm{mg}, 0.150 \mathrm{mmol}, 0.300$ equiv.), $62 \mathrm{~h}$. Isolated in $52 \%$ yield after purification by column chromatography (10:1 Hexane/EtOAc) as a light yellow oil.

${ }^{1} \mathrm{H}$ NMR $\left(\mathrm{CDCl}_{3}, 500 \mathrm{MHz}\right) \delta 7.27(\mathrm{~m}, 1 \mathrm{H}), 6.87(\mathrm{~m}, 1 \mathrm{H}), 4.18(\mathrm{q}, J=7.1 \mathrm{~Hz}, 2 \mathrm{H}), 3.95(\mathrm{~s}$, $3 \mathrm{H}), 3.62(\mathrm{~s}, 2 \mathrm{H}), 1.26(\mathrm{t}, J=7.2 \mathrm{~Hz}, 3 \mathrm{H})$;

${ }^{13} \mathrm{C}$ NMR $\left(\mathrm{CDCl}_{3}, 125 \mathrm{MHz}\right) \delta$ 170.1, $154.7(\mathrm{~d}, J=251 \mathrm{~Hz}), 145.5(\mathrm{~d}, J=13 \mathrm{~Hz}), 127.8$ (d, J = $41 \mathrm{~Hz}), 126.2(\mathrm{~d}, J=41 \mathrm{~Hz}), 122.7(\mathrm{~d}, J=14.7 \mathrm{~Hz}), 116.4(\mathrm{~d}, J=3.0 \mathrm{~Hz}), 61.5(\mathrm{~d}, J=4.3 \mathrm{~Hz}), 61.3$, $34.4(\mathrm{~d}, J=3.6 \mathrm{~Hz}), 14.2$;

${ }^{19}$ F NMR $\left(\mathrm{CDCl}_{3}, 377 \mathrm{MHz}\right) \delta$-130.6;

HRMS (LCMS ESI): calcd for $\mathrm{C}_{11} \mathrm{H}_{12} \mathrm{BrFNaO}_{3}[\mathrm{M}+\mathrm{Na}]^{+}$: 312.9846 . Found 312.9842.

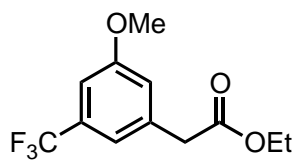

2s Prepared according to the General Procedure A from the corresponding neopentyl boronic ester (288 mg, $1.00 \mathrm{mmol}, 2.00$ equiv.) and mono-ethyl malonate $(66.1 \mathrm{mg}, 0.500$ mmol, 1.00 equiv.) using $\mathrm{Cu}(\mathrm{OTf})_{2}(54.3 \mathrm{mg}, 0.150 \mathrm{mmol}, 0.300$ equiv.), $48 \mathrm{~h}$. Isolated in $79 \%$ yield after purification by column chromatography (10:1 Hexane/EtOAc) as a colorless oil.

${ }^{1} \mathrm{H}$ NMR $\left(\mathrm{CDCl}_{3}, 500 \mathrm{MHz}\right) \delta 7.13(\mathrm{~m}, 1 \mathrm{H}), 7.02(\mathrm{~m}, 2 \mathrm{H}), 4.17(\mathrm{q}, J=7.0 \mathrm{~Hz}, 2 \mathrm{H}), 3.84(\mathrm{~s}$, $3 \mathrm{H}), 3.63(\mathrm{~s}, 2 \mathrm{H}), 1.26(\mathrm{t}, J=7.1 \mathrm{~Hz}, 3 \mathrm{H})$;

${ }^{13} \mathrm{C}$ NMR $\left(\mathrm{CDCl}_{3}, 125 \mathrm{MHz}\right) \delta$ 170.8, 159.9, 136.5, $132.0(\mathrm{q}, J=34 \mathrm{~Hz}), 123.9(\mathrm{q}, J=271 \mathrm{~Hz})$, 118.6, 118.4, 109.5, 61.2, 55.5, 41.2, 14.2;

${ }^{19} \mathrm{~F}$ NMR $\left(\mathrm{CDCl}_{3}, 377 \mathrm{MHz}\right) \delta-62.7$;

HRMS (EI): calcd for $\mathrm{C}_{12} \mathrm{H}_{13} \mathrm{~F}_{3} \mathrm{O}_{3}[\mathrm{M}]^{+}: 262.0817$. Found 262.0814.

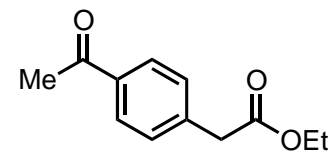

2t Prepared according to the General Procedure A from the corresponding neopentyl boronic ester (232 mg, $1.00 \mathrm{mmol}, 2.00$ equiv.) and mono-ethyl malonate (66.1 mg, 0.500 mmol, 1.00 equiv.) using $\mathrm{Cu}(\mathrm{OTf})_{2}(54.3 \mathrm{mg}, 0.150 \mathrm{mmol}, 0.300$ equiv.), $67 \mathrm{~h}$. Isolated in $75 \%$ yield after purification by column chromatography (6:1 to 1:1 Hexane/EtOAc) as a white solid. Spectroscopic data agreed with that reported. ${ }^{15}$ 


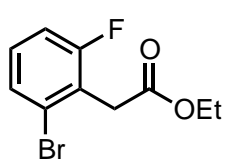

$2 \mathbf{u}$ Prepared according to the General Procedure A from the corresponding neopentyl boronic ester (430 mg, $1.50 \mathrm{mmol}, 3.00$ equiv.) and mono-ethyl malonate $(66.1 \mathrm{mg}, 0.500$ mmol, 1.00 equiv.) using $\mathrm{Cu}(\mathrm{OTf})_{2}(54.3 \mathrm{mg}, 0.150 \mathrm{mmol}, 0.300$ equiv.), $61 \mathrm{~h}$. Isolated in $54 \%$ yield after purification by column chromatography (30:1 to 4:1 Hexane/EtOAc) as a colorless oil.

${ }^{1} \mathrm{H}$ NMR $\left(\mathrm{CDCl}_{3}, 500 \mathrm{MHz}\right) \delta 7.38(\mathrm{~m}, 1 \mathrm{H}), 7.15(\mathrm{~m}, 1 \mathrm{H}), 7.04(\mathrm{~m}, 1 \mathrm{H}), 4.19(\mathrm{q}, J=7.4 \mathrm{~Hz}$, $2 \mathrm{H}), 3.86(\mathrm{~d}, J=1.9 \mathrm{~Hz}, 2 \mathrm{H}), 1.27(\mathrm{t}, J=7.2 \mathrm{~Hz}, 3 \mathrm{H})$;

${ }^{13} \mathrm{C}$ NMR $\left(\mathrm{CDCl}_{3}, 125 \mathrm{MHz}\right) \delta$ 169.6, $161.4(\mathrm{~d}, J=244 \mathrm{~Hz}), 129.6(\mathrm{~d}, J=9.4 \mathrm{~Hz}), 128.4(\mathrm{~d}, J=$ $4.4 \mathrm{~Hz}), 126.0(\mathrm{~d}, J=4.4 \mathrm{~Hz}), 123.0(\mathrm{~d}, J=18.9 \mathrm{~Hz}), 114.6(\mathrm{~d}, J=23.4 \mathrm{~Hz}), 61.3,34.7,14.2$;

${ }^{19}$ F NMR $\left(\mathrm{CDCl}_{3}, 469 \mathrm{MHz}\right) \delta$-111.3;

HRMS (LCMS ESI): calcd for $\mathrm{C}_{10} \mathrm{H}_{10} \mathrm{BrFNaO}_{2}[\mathrm{M}+\mathrm{Na}]^{+}: 282.9740$. Found 282.9743.

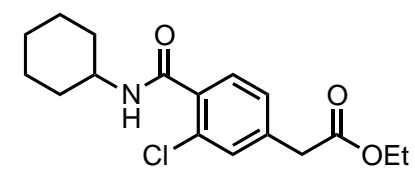

2w Prepared according to the General Procedure A from the corresponding neopentyl boronic ester (262 mg, $0.750 \mathrm{mmol}, 1.50$ equiv.) and mono-ethyl malonate (66.1 mg, 0.500 mmol, 1.00 equiv.) using $\mathrm{Cu}(\mathrm{OTf})_{2}(90.4 \mathrm{mg}, 0.250 \mathrm{mmol}, 0.500$ equiv.), $54 \mathrm{~h}$. Isolated in $55 \%$ yield after purification by column chromatography (4:1 to 1:1 Hexane/EtOAc) as a white solid.

${ }^{1} \mathrm{H} \mathrm{NMR}\left(\mathrm{CDCl}_{3}, 500 \mathrm{MHz}\right) \delta 7.59(\mathrm{~m}, 1 \mathrm{H}), 7.31(\mathrm{~m}, 1 \mathrm{H}), 7.21(\mathrm{~m}, 1 \mathrm{H}), 6.08(\mathrm{br}, 1 \mathrm{H}), 4.14(\mathrm{q}$, $J=7.1 \mathrm{~Hz}, 2 \mathrm{H}), 3.99(\mathrm{~m}, 1 \mathrm{H}), 3.58(\mathrm{~s}, 2 \mathrm{H}), 2.05-1.97(\mathrm{~m}, 2 \mathrm{H}), 1.77-1.68(\mathrm{~m}, 2 \mathrm{H}), 1.63(\mathrm{~m}, 1 \mathrm{H})$, $1.48-1.36(\mathrm{~m}, 2 \mathrm{H}), 1.31-1.15(\mathrm{~m}, 6 \mathrm{H})$;

${ }^{13} \mathrm{C}$ NMR $\left(\mathrm{CDCl}_{3}, 125 \mathrm{MHz}\right) \delta$ 170.6, 165.4, 137.7, 134.4, 131.0, 130.7, 130.4, 128.2, 61.3, 49.0, 40.8, 33.0, 25.6, 24.8, 14.3;

HRMS (LCMS ESI): calcd for $\mathrm{C}_{17} \mathrm{H}_{22} \mathrm{ClNNaO}_{3}[\mathrm{M}+\mathrm{Na}]^{+}$: 346.1180. Found 346.1185.

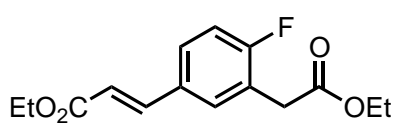

2v Prepared according to the General Procedure A from the corresponding neopentyl boronic ester $(230 \mathrm{mg}, 0.750 \mathrm{mmol}, 1.50$ equiv.) and mono-ethyl malonate $(66.1 \mathrm{mg}, 0.500$ mmol, 1.00 equiv.) using $\mathrm{Cu}(\mathrm{OTf})_{2}(54.3 \mathrm{mg}, 0.150 \mathrm{mmol}, 0.300$ equiv.), $60 \mathrm{~h}$. Isolated in $55 \%$ yield after purification by column chromatography (30:1 to 4:1 Hexane/EtOAc) as a colorless oil.

${ }^{1} \mathrm{H}$ NMR $\left(\mathrm{CDCl}_{3}, 500 \mathrm{MHz}\right) \delta 7.62(\mathrm{~d}, \mathrm{~J}=16 \mathrm{~Hz}, 1 \mathrm{H}), 7.45-7.42(\mathrm{~m}, 2 \mathrm{H}), 7.07(\mathrm{~m}, 1 \mathrm{H}), 6.35$ $(\mathrm{d}, \mathrm{J}=16 \mathrm{~Hz}, 1 \mathrm{H}), 4.26(\mathrm{q}, \mathrm{J}=7.2 \mathrm{~Hz}, 2 \mathrm{H}), 4.18(\mathrm{q}, \mathrm{J}=7.2 \mathrm{~Hz}, 2 \mathrm{H}), 3.67(\mathrm{~s}, 2 \mathrm{H}), 1.33(\mathrm{t}, \mathrm{J}=7.1 \mathrm{~Hz}$, $3 \mathrm{H}), 1.27(\mathrm{t}, \mathrm{J}=7.1 \mathrm{~Hz}, 3 \mathrm{H})$; 
${ }^{13} \mathrm{C}$ NMR $\left(\mathrm{CDCl}_{3}, 125 \mathrm{MHz}\right) \delta$ 170.3, 166.9, $162.2(\mathrm{~d}, \mathrm{~J}=260 \mathrm{~Hz}), 143.2,131.4,130.9,128.9$, 122.3, 118.3, 116.2, 61.3, 60.6, 34.5, 14.4, 14.2;

${ }^{19}$ F NMR $\left(\mathrm{CDCl}_{3}, 469 \mathrm{MHz}\right) \delta$-113.9;

HRMS (LCMS ESI): calcd for $\mathrm{C}_{15} \mathrm{H}_{17} \mathrm{FNaO}_{4}[\mathrm{M}+\mathrm{Na}]^{+}$: 303.1003. Found 303.1005.

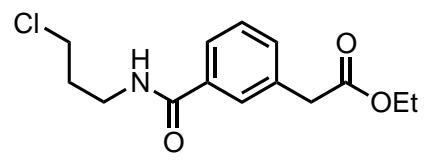

2x Prepared according to the General Procedure A from the corresponding neopentyl boronic ester (232 mg, $0.750 \mathrm{mmol}, 1.50$ equiv.) and mono-ethyl malonate (66.1 mg, 0.500 mmol, 1.00 equiv.) using $\mathrm{Cu}(\mathrm{OTf})_{2}(90.4 \mathrm{mg}, 0.250 \mathrm{mmol}, 0.500$ equiv.), $61 \mathrm{~h}$. Isolated in $70 \%$ yield after purification by column chromatography (3:2 to 2:3 Hexane/EtOAc) as a colorless oil.

${ }^{1} \mathrm{H} \mathrm{NMR}\left(\mathrm{CDCl}_{3}, 500 \mathrm{MHz}\right) \delta 7.69(\mathrm{~m}, 1 \mathrm{H}), 7.65(\mathrm{~m}, 1 \mathrm{H}), 7.45-7.38(\mathrm{~m}, 2 \mathrm{H}), 6.33(\mathrm{br}, 1 \mathrm{H})$, $4.16(\mathrm{q}, \mathrm{J}=7.2 \mathrm{~Hz}, 2 \mathrm{H}), 3.68-3.60(\mathrm{~m}, 6 \mathrm{H}), 2.13$ (quint, J = 6.4 Hz, 2H), $1.26(\mathrm{t}, \mathrm{J}=7.1 \mathrm{~Hz}, 3 \mathrm{H})$;

${ }^{13} \mathrm{C}$ NMR $\left(\mathrm{CDCl}_{3}, 125 \mathrm{MHz}\right) \delta 171.2,167.5,134.8$ (2C), 132.6, 128.9, 127.9, 125.6, 61.1, 42.7, 41.2, 37.7, 32.1, 14.2;

HRMS (LCMS ESI): calcd for $\mathrm{C}_{14} \mathrm{H}_{18} \mathrm{ClNNaO}_{3}[\mathrm{M}+\mathrm{Na}]^{+}$: 306.0867. Found 306.0866.

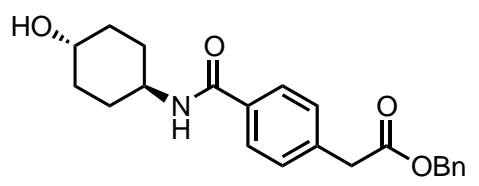

2y Prepared according to the General Procedure A from the corresponding neopentyl boronic ester (218 mg, $0.750 \mathrm{mmol}, 1.50$ equiv.) and mono-benzyl malonate (66.1 mg, 0.500 mmol, 1.00 equiv.) using $\mathrm{Cu}(\mathrm{OTf})_{2}(90.4 \mathrm{mg}, 0.250 \mathrm{mmol}, 0.500$ equiv.), $48 \mathrm{~h}$. Crude NMR shows 54\% NMR yield using trimethoxybenzene as internal standard. Isolated in 39\% yield after purification by column chromatography $\left(6 \%\right.$ to $\left.7 \% \mathrm{MeOH} / \mathrm{CH}_{2} \mathrm{Cl}_{2}\right)$ as a white solid.

${ }^{1} \mathrm{H}$ NMR $\left(\mathrm{CDCl}_{3}, 500 \mathrm{MHz}\right) \delta 7.71-7.69(\mathrm{~m}, 2 \mathrm{H}), 7.37-7.30(\mathrm{~m}, 7 \mathrm{H}), 5.85(\mathrm{br}, 1 \mathrm{H}), 5.13(\mathrm{~s}$, $2 \mathrm{H}), 3.97(\mathrm{~m}, 1 \mathrm{H}), 3.71(\mathrm{~s}, 2 \mathrm{H}), 3.66(\mathrm{~m}, 1 \mathrm{H}), 2.16-2.09(\mathrm{~m}, 2 \mathrm{H}), 2.06-2.0(\mathrm{~m}, 2 \mathrm{H}), 1.6-1.4(\mathrm{~m}$, $3 \mathrm{H}), 1.35-1.25(\mathrm{~m}, 2 \mathrm{H})$;

${ }^{13} \mathrm{C}$ NMR $\left(\mathrm{CDCl}_{3}, 125 \mathrm{MHz}\right) \delta 170.8,166.6,137.4,135.7,133.8,129.6,128.6,128.4,128.3$, 127.2, 69.9, 66.9, 48.1, 41.2, 34.1, 31.0;

HRMS (LCMS ESI): calcd for $\mathrm{C}_{22} \mathrm{H}_{25} \mathrm{NNaO}_{4}[\mathrm{M}+\mathrm{Na}]^{+}:$390.1676. Found 390.1674. 
<smiles>CCOC(=O)Cc1cccc(C(=O)N2CCCC(C(=O)OCC)C2)c1</smiles>

$2 z$ Prepared according to the General Procedure A from the corresponding neopentyl boronic ester (280 mg, $0.750 \mathrm{mmol}, 1.50$ equiv.) and mono-ethyl malonate (66.1 mg, 0.500 mmol, 1.00 equiv.) using $\mathrm{Cu}(\mathrm{OTf})_{2}(54.3 \mathrm{mg}, 0.150 \mathrm{mmol}, 0.300$ equiv.), $67 \mathrm{~h}$. Isolated in $63 \%$ yield after purification by column chromatography (4:1 Hexane/EtOAc to EtOAc) as a colorless oil.

${ }^{1} \mathrm{H}$ NMR (DMSO-d6, $\left.400 \mathrm{MHz}, 120{ }^{\circ} \mathrm{C}\right) \delta 7.34(\mathrm{~m}, 1 \mathrm{H}), 7.30(\mathrm{~m}, 1 \mathrm{H}), 7.20-7.25(\mathrm{~m}, 2 \mathrm{H})$, $4.01-4.11(\mathrm{~m}, 4 \mathrm{H}), 3.98(\mathrm{~m}, 1 \mathrm{H}), 3.67(\mathrm{~m}, 1 \mathrm{H}), 3.64(\mathrm{~s}, 2 \mathrm{H}), 3.22(\mathrm{dd}, \mathrm{J}=10 \mathrm{~Hz}, 13.2 \mathrm{~Hz}, 1 \mathrm{H})$, $3.09(\mathrm{~m}, 1 \mathrm{H}), 2.51(\mathrm{~m}, 1 \mathrm{H}), 1.95(\mathrm{~m}, 1 \mathrm{H}), 1.61-1.72(\mathrm{~m}, 2 \mathrm{H}), 1.45(\mathrm{~m}, 1 \mathrm{H}), 1.12-1.17(\mathrm{~m}, 6 \mathrm{H})$;

${ }^{13} \mathrm{C}$ NMR (DMSO- $\mathrm{d}_{6}, 100 \mathrm{MHz}, 120{ }^{\circ} \mathrm{C}$ ) $\delta$ 171.7, 169.9, 168.6, 135.9, 134.1, 129.4, 127.6, 126.8, 124.5, 59.6, 59.3, 45.3, 44.2, 40.2, 26.0, 23.1, 13.3, 13.2 (one peak missing, obscured by solvent signal);

HRMS (LCMS ESI): calcd for $\mathrm{C}_{19} \mathrm{H}_{25} \mathrm{NNaO}_{5}[\mathrm{M}+\mathrm{Na}]^{+}: 370.1625$. Found 370.1631.

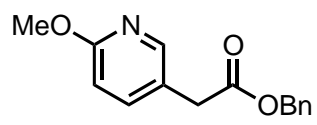

3a Prepared according to the General Procedure from the corresponding neopentyl boronic ester (165.8 mg, $0.75 \mathrm{mmol}, 1.50$ equiv.) and mono-benzyl malonate $(97.1 \mathrm{mg}, 0.500$ mmol, 1.00 equiv.) using $\mathrm{Cu}(\mathrm{OTf})_{2}(90.4 \mathrm{mg}, 0.250 \mathrm{mmol}, 0.500$ equiv.), 46h. Isolated in $68 \%$ yield after purification by column chromatography (4:1 Hexane/EtOAc) as a colorless oil.

${ }^{1} \mathrm{H}$ NMR $\left(\mathrm{CDCl}_{3}, 700 \mathrm{MHz}\right) \delta 8.05(\mathrm{~d}, J=2.1 \mathrm{~Hz}, 1 \mathrm{H}), 7.52(\mathrm{dd}, J=9.1,2.1 \mathrm{~Hz}, 1 \mathrm{H}), 7.29-$ $7.38(\mathrm{~m}, 5 \mathrm{H}), 6.72(\mathrm{~d}, J=9.1 \mathrm{~Hz}, 1 \mathrm{H}), 5.14(\mathrm{~s}, 2 \mathrm{H}), 3.92(\mathrm{~s}, 3 \mathrm{H}), 3.59(\mathrm{~s}, 2 \mathrm{H})$;

${ }^{13} \mathrm{C}$ NMR $\left(\mathrm{CDCl}_{3}, 176 \mathrm{MHz}\right) \delta 171.2,163.6,147.1,139.8,135.8,128.7,128.5,128.4,122.4$, $110.9,67.0,53.6,37.7$;

HRMS (LCMS ESI): calcd for $\mathrm{C}_{15} \mathrm{H}_{16} \mathrm{NO}_{3}[\mathrm{M}+\mathrm{H}]^{+}:$258.1125. Found 258.1127.

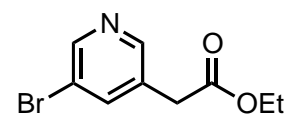

$3 \mathrm{~b}$ Prepared according to the General Procedure from the corresponding neopentyl boronic ester (202.4 mg, $0.75 \mathrm{mmol}, 1.50$ equiv.) and mono-ethyl malonate (66.1 mg, 0.500 mmol, 1.00 equiv.) using $\mathrm{Cu}(\mathrm{OTf})_{2}(90.4 \mathrm{mg}, 0.250 \mathrm{mmol}, 0.500$ equiv.), $70 \mathrm{~h}$. Isolated in $47 \%$ yield after purification by column chromatography (2:1 Hexane/EtOAc) as a colorless oil.

${ }^{1} \mathrm{H} \mathrm{NMR}\left(\mathrm{CDCl}_{3}, 700 \mathrm{MHz}\right) \delta 8.60(\mathrm{br} \mathrm{s}, 1 \mathrm{H}), 8.44(\mathrm{br} \mathrm{s}, 1 \mathrm{H}), 7.81(\mathrm{t}, J=2.1 \mathrm{~Hz}, 1 \mathrm{H}), 4.18(\mathrm{q}$, $J=7.0 \mathrm{~Hz}, 2 \mathrm{H}), 3.60(\mathrm{~s}, 2 \mathrm{H}), 1.27(\mathrm{t}, J=7.0 \mathrm{~Hz}, 3 \mathrm{H})$;

${ }^{13} \mathrm{C}$ NMR $\left(\mathrm{CDCl}_{3}, 176 \mathrm{MHz}\right) \delta 170.2,149.8,148.6,139.6,131.6,120.8,61.6,38.1,14.3$;

HRMS (LCMS ESI): calcd for $\mathrm{C}_{9} \mathrm{H}_{11} \mathrm{BrNO}_{2}[\mathrm{M}+\mathrm{H}]^{+}$: 243.9968. Found 243.9965. 


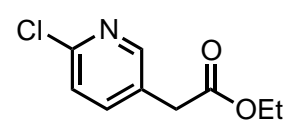

3c Prepared according to the General Procedure from the corresponding neopentyl boronic ester (169.1 mg, $0.75 \mathrm{mmol}, 1.50$ equiv.) and mono-ethyl malonate ( $66.1 \mathrm{mg}, 0.500$ mmol, 1.00 equiv.) using $\mathrm{Cu}(\mathrm{OTf})_{2}(90.4 \mathrm{mg}, 0.250 \mathrm{mmol}, 0.500$ equiv.), $70 \mathrm{~h}$. Isolated in $67 \%$ yield after purification by column chromatography (4:1 Hexane/EtOAc) as a colorless oil.

${ }^{1} \mathrm{H}$ NMR $\left(\mathrm{CDCl}_{3}, 700 \mathrm{MHz}\right) \delta 8.29(\mathrm{~s}, 1 \mathrm{H}), 7.62(\mathrm{dd}, J=8.4,3.2 \mathrm{~Hz}, 1 \mathrm{H}), 7.30(\mathrm{~d}, J=8.4 \mathrm{~Hz}$, $1 \mathrm{H}) 4.17(\mathrm{q}, J=7.0 \mathrm{~Hz}, 2 \mathrm{H}), 3.60(\mathrm{~s}, 2 \mathrm{H}), 1.26(\mathrm{t}, J=7.2 \mathrm{~Hz}, 3 \mathrm{H})$;

${ }^{13} \mathrm{C}$ NMR $\left(\mathrm{CDCl}_{3}, 176 \mathrm{MHz}\right) \delta 170.4,150.5,150.2,139.8,128.9,124.2,61.5,37.8,14.3$;

HRMS (LCMS ESI): calcd for $\mathrm{C}_{9} \mathrm{H}_{11} \mathrm{ClNO}_{2}[\mathrm{M}+\mathrm{H}]^{+}: 200.0473$. Found 200.0469.

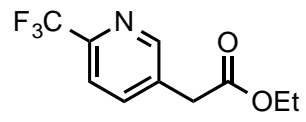

3d Prepared according to the General Procedure from the corresponding neopentyl boronic ester (194.3 mg, $0.75 \mathrm{mmol}, 1.50$ equiv.) and mono-ethyl malonate $(66.1 \mathrm{mg}, 0.500$ mmol, 1.00 equiv.) using $\mathrm{Cu}(\mathrm{OTf})_{2}(90.4 \mathrm{mg}, 0.25 \mathrm{mmol}, 0.500$ equiv.), $44 \mathrm{~h}$. Isolated in $77 \%$ yield after purification by column chromatography (4:1 Hexane/EtOAc) as a colorless oil.

${ }^{1} \mathrm{H}$ NMR $\left(\mathrm{CDCl}_{3}, 700 \mathrm{MHz}\right) \delta 8.64(\mathrm{~s}, 1 \mathrm{H}), 7.83(\mathrm{~m}, 1 \mathrm{H}), 7.66(\mathrm{~d}, J=8.4 \mathrm{~Hz}, 1 \mathrm{H}), 4.19(\mathrm{q}, J=$ $7.0 \mathrm{~Hz}, 2 \mathrm{H}), 3.71(\mathrm{~s}, 2 \mathrm{H}), 1.27(\mathrm{t}, J=7.0 \mathrm{~Hz}, 3 \mathrm{H})$;

${ }^{13} \mathrm{C}$ NMR $\left(\mathrm{CDCl}_{3}, 176 \mathrm{MHz}\right) \delta$ 170.0, 150.7, $147.2(\mathrm{q}, J=34.8 \mathrm{~Hz}), 138.3,133.3,121.7(\mathrm{q}, J=$ $274.5 \mathrm{~Hz}) 120.4,61.7,38.4,14.3$;

${ }^{19} \mathrm{~F}$ NMR $\left(\mathrm{CDCl}_{3}, 377 \mathrm{MHz}\right) \delta-68.00$;

HRMS (LCMS ESI): calcd for $\mathrm{C}_{10} \mathrm{H}_{11} \mathrm{~F}_{3} \mathrm{NO}_{2}[\mathrm{M}+\mathrm{H}]^{+}:$234.0736. Found 234.0737.

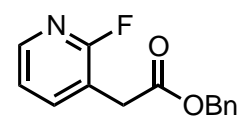

3e Prepared according to the General Procedure from the corresponding neopentyl boronic ester (156.8 mg, $0.75 \mathrm{mmol}, 1.50$ equiv.) and mono-benzyl malonate $(97.1 \mathrm{mg}, 0.500$ mmol, 1.00 equiv.) using $\mathrm{Cu}(\mathrm{OTf})_{2}(90.4 \mathrm{mg}, 0.250 \mathrm{mmol}, 0.500$ equiv.), $53 \mathrm{~h}$. Isolated in $53 \%$ yield after purification by column chromatography (4:1 Hexane/EtOAc) as a colorless oil.

${ }^{1} \mathrm{H}$ NMR $\left(\mathrm{CDCl}_{3}, 700 \mathrm{MHz}\right) \delta 8.15(\mathrm{~d}, J=4.9 \mathrm{~Hz}, 1 \mathrm{H}), 7.71(\mathrm{~m}, 1 \mathrm{H}), 7.31-7.38(\mathrm{~m}, 5 \mathrm{H}), 7.16$ (m, 1H), $5.17(\mathrm{~s}, 2 \mathrm{H}), 3.72(\mathrm{~s}, 2 \mathrm{H})$;

${ }^{13} \mathrm{C}$ NMR $\left(\mathrm{CDCl}_{3}, 176 \mathrm{MHz}\right) \delta 169.8,162.0(\mathrm{~d}, J=238.8 \mathrm{~Hz}), 146.8(\mathrm{~d}, J=14.8 \mathrm{~Hz}), 142.0(\mathrm{~d}, J$ $=4.8 \mathrm{~Hz}), 135.5,128.7,128.5,128.4,121.6(\mathrm{~d}, J=4.4 \mathrm{~Hz}), 116.6(\mathrm{~d}, J=31.1 \mathrm{~Hz}), 67.2,34.3(\mathrm{~d}, J=$ $1.9 \mathrm{~Hz})$;

${ }^{19}$ F NMR $\left(\mathrm{CDCl}_{3}, 376 \mathrm{MHz}\right) \delta-71.56$;

HRMS (LCMS ESI): calcd for $\mathrm{C}_{14} \mathrm{H}_{13} \mathrm{FNO}_{2}[\mathrm{M}+\mathrm{H}]^{+}:$246.0925. Found 246.0921. 


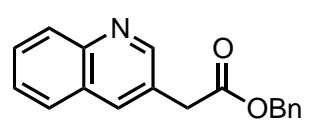

3f Prepared according to the General Procedure from the corresponding neopentyl boronic ester (180.8 mg, $0.75 \mathrm{mmol}, 1.50$ equiv.) and mono-benzyl malonate $(97.1 \mathrm{mg}, 0.500$ mmol, 1.00 equiv.) using $\mathrm{Cu}(\mathrm{OTf})_{2}(90.4 \mathrm{mg}, 0.250 \mathrm{mmol}, 0.500$ equiv.), $51 \mathrm{~h}$. Isolated in $73 \%$ yield after purification by column chromatography (Hexane/EtOAc gradient with $6 \% \mathrm{NEt}_{3}$ ) as a white solid.

${ }^{1} \mathrm{H}$ NMR $\left(\mathrm{CDCl}_{3}, 700 \mathrm{MHz}\right) \delta 8.85(\mathrm{~d}, J=2.1 \mathrm{~Hz}, 1 \mathrm{H}), 8.10(\mathrm{~d}, J=8.4 \mathrm{~Hz}, 1 \mathrm{H}), 8.07(\mathrm{br} \mathrm{s}$, $1 \mathrm{H}), 7.78(\mathrm{~d}, J=8.4 \mathrm{~Hz}, 1 \mathrm{H}), 7.70(\mathrm{~m}, 1 \mathrm{H}) 7.55(\mathrm{~m}, 1 \mathrm{H}), 7.30-7.37(\mathrm{~m}, 5 \mathrm{H}), 5.17(\mathrm{~s}, 2 \mathrm{H}), 3.86(\mathrm{~s}$, $2 \mathrm{H})$;

${ }^{13} \mathrm{C}$ NMR $\left(\mathrm{CDCl}_{3}, 176 \mathrm{MHz}\right) \delta 170.7,151.8,147.5,136.0,135.6,129.5,129.4,128.8,128.6$, 128.5, 128.0, 127.7, 127.0, 126.9, 67.2, 38.8;

HRMS (LCMS ESI): calcd for $\mathrm{C}_{18} \mathrm{H}_{16} \mathrm{NO}_{2}[\mathrm{M}+\mathrm{H}]^{+}: 278.1176$. Found 278.1171.

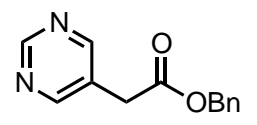

3g Prepared according to the General Procedure, with the modification of using 1,2-DCE as the solvent, from the corresponding neopentyl boronic ester $(144.0 \mathrm{mg}, 0.75 \mathrm{mmol}, 1.50$ equiv.) and mono-benzyl malonate $\left(97.1 \mathrm{mg}, 0.500 \mathrm{mmol}, 1.00\right.$ equiv.) using $\mathrm{Cu}(\mathrm{OTf})_{2}(90.4$ $\mathrm{mg}, 0.250 \mathrm{mmol}, 0.500$ equiv.), 31h. The solvent was removed in vacuo. The crude residue was purified by silica gel chromatography $(25: 1 \mathrm{DCM} / \mathrm{MeOH})$. Isolated in $61 \%$ yield as a colorless oil.

${ }^{1} \mathrm{H}$ NMR $\left(\mathrm{CDCl}_{3}, 700 \mathrm{MHz}\right) \delta 9.14(\mathrm{~s}, 1 \mathrm{H}), 8.680(\mathrm{~s}, 2 \mathrm{H}), 7.38-7.32(\mathrm{~m}, 5 \mathrm{H}), 5.16(\mathrm{~s}, 2 \mathrm{H})$, $3.67(\mathrm{~s}, 2 \mathrm{H})$;

${ }^{13} \mathrm{C}$ NMR $\left(\mathrm{CDCl}_{3}, 176 \mathrm{MHz}\right) \delta$ 170.0, 157.8, 157.5, 135.3, 128.8, 128.7, 128.5, 127.9, 67.5, 36.0;

HRMS (LCMS ESI): calcd for $\mathrm{C}_{13} \mathrm{H}_{13} \mathrm{~N}_{2} \mathrm{O}_{2}[\mathrm{M}+\mathrm{H}]^{+}: 229.0972$. Found 229.0972 .

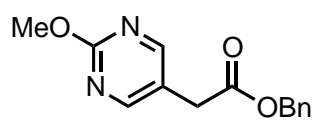

3h Prepared according to the General Procedure from the corresponding neopentyl boronic ester ( $166.5 \mathrm{mg}, 0.75 \mathrm{mmol}, 1.50$ equiv.) and mono-benzyl malonate $(97.1 \mathrm{mg}, 0.500$ mmol, 1.00 equiv.) using $\mathrm{Cu}(\mathrm{OTf})_{2}(90.4 \mathrm{mg}, 0.250 \mathrm{mmol}, 0.500$ equiv.), $48 \mathrm{~h}$. Isolated in $65 \%$ yield after purification by column chromatography (1:1 Hexane/EtOAc) as a colorless oil.

${ }^{1} \mathrm{H}$ NMR $\left(\mathrm{CDCl}_{3}, 700 \mathrm{MHz}\right) \delta 8.44(\mathrm{~s}, 2 \mathrm{H}), 7.32$ - $7.37(\mathrm{~m}, 5 \mathrm{H}), 5.15(\mathrm{~s}, 2 \mathrm{H}), 4.00(\mathrm{~s}, 3 \mathrm{H}), 3.59$ (s, 2H);

${ }^{13} \mathrm{C}$ NMR $\left(\mathrm{CDCl}_{3}, 176 \mathrm{MHz}\right) \delta 170.3,165.1,159.8,135.4,128.8,128.7,128.5,120.8,67.4,55.1$, 35.1;

HRMS (LCMS ESI): calcd for $\mathrm{C}_{14} \mathrm{H}_{15} \mathrm{~N}_{2} \mathrm{O}_{3}[\mathrm{M}+\mathrm{H}]^{+}:$259.1077. Found 259.1080. 


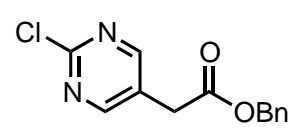

3i Prepared according to the General Procedure from the corresponding neopentyl boronic ester (169.9 mg, $0.75 \mathrm{mmol}, 1.50$ equiv.) and mono-benzyl malonate $(97.1 \mathrm{mg}, 0.500$ mmol, 1.00 equiv.) using $\mathrm{Cu}(\mathrm{OTf})_{2}(90.4 \mathrm{mg}, 0.250 \mathrm{mmol}, 0.500$ equiv.), $48 \mathrm{~h}$. Isolated in $53 \%$ yield after purification by column chromatography $\left(10: 1 \mathrm{CH}_{2} \mathrm{Cl}_{2} / \mathrm{Et}_{2} \mathrm{O}\right)$ as a pale yellow solid.

${ }^{1} \mathrm{H}$ NMR $\left(\mathrm{CDCl}_{3}, 700 \mathrm{MHz}\right) \delta 8.57(\mathrm{~s}, 2 \mathrm{H}), 7.32-7.39(\mathrm{~m}, 5 \mathrm{H}), 5.17(\mathrm{~s}, 2 \mathrm{H}), 3.67(\mathrm{~s}, 2 \mathrm{H})$;

${ }^{13} \mathrm{C}$ NMR $\left(\mathrm{CDCl}_{3}, 176 \mathrm{MHz}\right) \delta 169.3,160.6,160.2,135.1,128.9,128.9,128.6,126.4,67.7,35.2$;

HRMS (LCMS ESI): calcd for $\mathrm{C}_{13} \mathrm{H}_{12} \mathrm{ClN}_{2} \mathrm{O}_{2}[\mathrm{M}+\mathrm{H}]^{+}$: 263.0582. Found 263.0578.

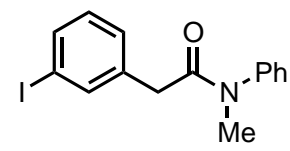

4a Prepared according to the General Procedure A from the corresponding neopentyl boronic ester (316 mg, $1.00 \mathrm{mmol}, 2.00$ equiv.) and 3-(methyl(phenyl)amino)-3-oxopropanoic acid (96.6 mg, $0.500 \mathrm{mmol}, 1.00$ equiv.) using $\mathrm{Cu}(\mathrm{OTf})_{2}(54.3 \mathrm{mg}, 0.150 \mathrm{mmol}, 0.300$ equiv.), 61 h. Isolated in $84 \%$ yield after purification by column chromatography (4:1 to 2:1 Hexane/EtOAc) as a colorless oil.

${ }^{1} \mathrm{H}$ NMR $\left(\mathrm{CDCl}_{3}, 500 \mathrm{MHz}\right) \delta 7.52(\mathrm{~m}, 1 \mathrm{H}), 7.44-7.35(\mathrm{~m}, 3 \mathrm{H}), 7.32(\mathrm{~m}, 1 \mathrm{H}), 7.12$ - $7.10(\mathrm{~m}$, $2 \mathrm{H}), 7.06(\mathrm{~m}, 1 \mathrm{H}), 6.97(\mathrm{~m}, 1 \mathrm{H}), 3.39(\mathrm{~s}, 2 \mathrm{H}), 3.27(\mathrm{~s}, 3 \mathrm{H})$;

${ }^{13} \mathrm{C}$ NMR $\left(\mathrm{CDCl}_{3}, 125 \mathrm{MHz}\right) \delta 170.3,143.8,138.1,137.7,135.7,130.0,129.8,128.4,128.2$, 127.7, 94.2, 40.6, 37.7;

HRMS (LCMS ESI): calcd for $\mathrm{C}_{15} \mathrm{H}_{14} \mathrm{INNaO}[\mathrm{M}+\mathrm{Na}]^{+}: 374.0012$. Found 374.0015.

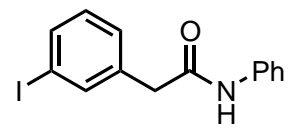

4b Prepared according to the General Procedure A from the corresponding neopentyl boronic ester ( $237 \mathrm{mg}, 0.750 \mathrm{mmol}, 1.50$ equiv.) and 3-oxo-3-(phenylamino)propanoic acid (89.6 mg, $0.500 \mathrm{mmol}, 1.00$ equiv.) using $\mathrm{Cu}(\mathrm{OTf})_{2}(54.3 \mathrm{mg}, 0.150 \mathrm{mmol}, 0.300$ equiv.), $63 \mathrm{~h}$. Isolated in $43 \%$ yield after purification by column chromatography (6:1 to 4:1 Hexane/EtOAc) as a white solid.

${ }^{1} \mathrm{H} \mathrm{NMR}\left(\mathrm{CDCl}_{3}, 500 \mathrm{MHz}\right) \delta 7.72(\mathrm{~m}, 1 \mathrm{H}), 7.69(\mathrm{~m}, 1 \mathrm{H}), 7.48-7.44(\mathrm{~m}, 2 \mathrm{H}), 7.36-7.30(\mathrm{~m}$, $3 \mathrm{H}), 7.17-7.10(\mathrm{~m}, 3 \mathrm{H}), 3.68(\mathrm{~s}, 2 \mathrm{H})$;

${ }^{13} \mathrm{C}$ NMR $\left(\mathrm{CDCl}_{3}, 125 \mathrm{MHz}\right) \delta 168.2,138.4,137.5,136.8,136.7,130.8,129.1,128.7,124.7$, 120.0, 95.0, 44.2;

HRMS (LCMS ESI): calcd for $\mathrm{C}_{14} \mathrm{H}_{12} \mathrm{INNaO}[\mathrm{M}+\mathrm{Na}]^{+}:$359.9856. Found 359.9858.

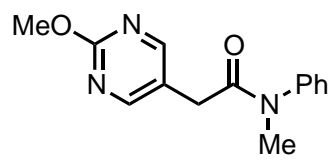

4c Prepared according to the General Procedure from the corresponding neopentyl boronic ester (166.5 mg, $0.75 \mathrm{mmol}, 1.50$ equiv.) and 3-(methyl(phenyl)amino)-3- 
oxopropanoic acid (96.6 mg, $0.500 \mathrm{mmol}, 1.00$ equiv.) using $\mathrm{Cu}(\mathrm{OTf})_{2}(90.4 \mathrm{mg}, 0.150 \mathrm{mmol}$, 0.500 equiv.), $43 \mathrm{~h}$. Isolated in $49 \%$ yield after purification by column chromatography (1:4 Hexane/EtOAc) as a white solid.

${ }^{1} \mathrm{H}$ NMR $\left(\mathrm{CDCl}_{3}, 700 \mathrm{MHz}\right) \delta 8.23(\mathrm{~s}, 2 \mathrm{H}), 7.46-7.48(\mathrm{~m}, 2 \mathrm{H}), 7.40-7.42(\mathrm{~m}, 1 \mathrm{H}), 7.18(\mathrm{~d}, J$ $=7.7 \mathrm{~Hz}, 2 \mathrm{H}), 3.97(\mathrm{~s}, 3 \mathrm{H}), 3.33(\mathrm{~s}, 2 \mathrm{H}), 3.28(\mathrm{~s}, 3 \mathrm{H})$;

${ }^{13} \mathrm{C}$ NMR $\left(\mathrm{CDCl}_{3}, 176 \mathrm{MHz}\right) \delta$ 169.7, 164.8, 159.7, 143.6, 130.3, 128.6, 127.6, 122.2, 55.0, 37.8, 34.8;

HRMS (LCMS ESI): calcd for $\mathrm{C}_{14} \mathrm{H}_{16} \mathrm{~N}_{3} \mathrm{O}_{2}[\mathrm{M}+\mathrm{H}]^{+}:$258.1237. Found 258.1237.

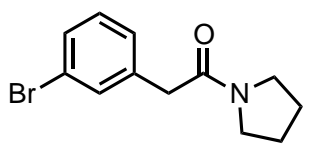

4d Prepared according to the General Procedure A from the corresponding neopentyl boronic ester (269 mg, $1.00 \mathrm{mmol}, 2.00$ equiv.) and 3-oxo-3-(pyrrolidin-1-yl)propanoic acid (78.6 mg, $0.500 \mathrm{mmol}, 1.00$ equiv.) using $\mathrm{Cu}(\mathrm{OTf})_{2}(54.3 \mathrm{mg}, 0.150 \mathrm{mmol}, 0.300$ equiv.), $70 \mathrm{~h}$. Isolated in $58 \%$ yield after purification by column chromatography $(0 \%$ to $10 \%$ $\mathrm{MeOH} / \mathrm{EtOAc}$ ) as a light yellow oil.

${ }^{1} \mathrm{H}$ NMR $\left(\mathrm{CDCl}_{3}, 400 \mathrm{MHz}\right) \delta 7.42(\mathrm{~m}, 1 \mathrm{H}), 7.35(\mathrm{~m}, 1 \mathrm{H}), 7.22-7.12(\mathrm{~m}, 2 \mathrm{H}), 3.59(\mathrm{~s}, 2 \mathrm{H})$, $3.48-3.39(\mathrm{~m}, 4 \mathrm{H}), 1.95-1.79(\mathrm{~m}, 4 \mathrm{H})$;

${ }^{13} \mathrm{C}$ NMR $\left(\mathrm{CDCl}_{3}, 125 \mathrm{MHz}\right) \delta$ 168.6, 137.2, 132.0, 130.0, 129.8, 127.7, 122.5, 46.9, 46.1, 14.6, 26.2, 24.4;

HRMS (LCMS ESI): calcd for $\mathrm{C}_{12} \mathrm{H}_{14} \mathrm{BrNNaO}[\mathrm{M}+\mathrm{Na}]^{+}: 290.0151$. Found 290.0155.

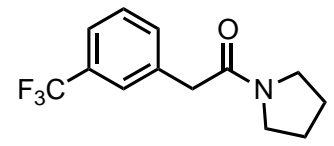

4e Prepared according to the General Procedure A from the corresponding neopentyl boronic ester (258 mg, $1.00 \mathrm{mmol}, 2.00$ equiv.) and 3-oxo-3-(pyrrolidin-1-yl)propanoic acid (78.6 mg, $0.500 \mathrm{mmol}, 1.00$ equiv.) using $\mathrm{Cu}(\mathrm{OTf})_{2}(54.3 \mathrm{mg}, 0.150 \mathrm{mmol}, 0.300$ equiv.), $72 \mathrm{~h}$. Isolated in $66 \%$ yield after purification by column chromatography (EtOAc) as a light yellow solid.

${ }^{1} \mathrm{H}$ NMR $\left(\mathrm{CDCl}_{3}, 500 \mathrm{MHz}\right) \delta 7.52-7.49(\mathrm{~m}, 3 \mathrm{H}), 7.43(\mathrm{~m}, 1 \mathrm{H}), 3.70(\mathrm{~s}, 2 \mathrm{H}), 3.50(\mathrm{t}, J=7.0$ $\mathrm{Hz}, 2 \mathrm{H}$ ), $3.45(\mathrm{t}, J=6.8 \mathrm{~Hz}, 2 \mathrm{H}$ ), 1.96 (quint, $J=6.8 \mathrm{~Hz}, 2 \mathrm{H}$ ), 1.86 (quint, $J=7.0 \mathrm{~Hz}, 2 \mathrm{H}$ );

${ }^{13} \mathrm{C}$ NMR $\left(\mathrm{CDCl}_{3}, 125 \mathrm{MHz}\right) \delta 168.6,136.0,132.7,130.8(\mathrm{q}, J=32 \mathrm{~Hz}), 129.0,125.9,124.1(\mathrm{q}, J$ $=272 \mathrm{~Hz}), 123.7,47.0,46.1,41.7,26.2,24.4$;

${ }^{19} \mathrm{~F}$ NMR $\left(\mathrm{CDCl}_{3}, 377 \mathrm{MHz}\right) \delta-62.6$;

HRMS (LCMS ESI): calcd for $\mathrm{C}_{13} \mathrm{H}_{14} \mathrm{~F}_{3} \mathrm{NNaO}[\mathrm{M}+\mathrm{Na}]^{+}: 280.0920$. Found 280.0919 .

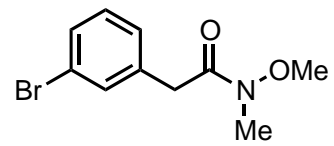

4f Prepared according to the General Procedure A from the corresponding neopentyl boronic ester (269 mg, $1.00 \mathrm{mmol}, 2.00$ equiv.) and 3-(methoxy(methyl)amino)-3- 
oxopropanoic acid (73.6 mg, $0.500 \mathrm{mmol}, 1.00$ equiv.) using $\mathrm{Cu}(\mathrm{OTf}) 2$ ( $54.3 \mathrm{mg}, 0.150 \mathrm{mmol}$, 0.300 equiv.), $68 \mathrm{~h}$. Isolated in $70 \%$ yield after purification by column chromatography (4:1 to 1:1 Hexane/EtOAc) as a light yellow oil.

${ }^{1} \mathrm{H}$ NMR $\left(\mathrm{CDCl}_{3}, 400 \mathrm{MHz}\right) \delta 7.44(\mathrm{~m}, 1 \mathrm{H}), 7.36(\mathrm{~m}, 1 \mathrm{H}), 7.23-7.13(\mathrm{~m}, 2 \mathrm{H}), 3.72(\mathrm{~s}, 2 \mathrm{H})$, $3.62(\mathrm{~s}, 3 \mathrm{H}), 3.18(\mathrm{~s}, 3 \mathrm{H})$;

${ }^{13} \mathrm{C}$ NMR $\left(\mathrm{CDCl}_{3}, 125 \mathrm{MHz}\right) \delta 171.5,137.1,132.3,129.92,129.89,128.0,122.4,61.3,38.7$, 32.2;

HRMS (LCMS ESI): calcd for $\mathrm{C}_{10} \mathrm{H}_{12} \mathrm{BrNNaO}_{2}[\mathrm{M}+\mathrm{Na}]^{+}$: 279.9944. Found 279.9949.

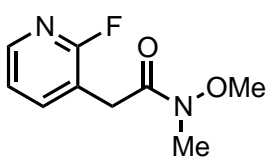

$4 \mathrm{~g}$ Prepared according to the General Procedure from the corresponding neopentyl boronic ester (159.8 mg, $0.75 \mathrm{mmol}, 1.50$ equiv.) and 3-(methoxy(methyl)amino)-3oxopropanoic acid (73.6 mg, $0.500 \mathrm{mmol}, 1.00$ equiv.) using $\mathrm{Cu}(\mathrm{OTf}) 2$ (90.4 mg, $0.250 \mathrm{mmol}$, 0.500 equiv.), $48 \mathrm{~h}$. Isolated in $51 \%$ yield after purification by column chromatography (Hexane/EtOAc gradient with $1 \% \mathrm{NEt}_{3}$ ) as a colorless oil.

${ }^{1} \mathrm{H}$ NMR $\left(\mathrm{CDCl}_{3}, 700 \mathrm{MHz}\right) \delta 8.12(\mathrm{~d}, J=3.5 \mathrm{~Hz}, 1 \mathrm{H}), 7.74(\mathrm{t}, J=8.1 \mathrm{~Hz}, 1 \mathrm{H}), 7.15-7.17(\mathrm{~m}$, $1 \mathrm{H}), 3.80(\mathrm{~s}, 2 \mathrm{H}), 3.73(\mathrm{~s}, 3 \mathrm{H}), 3.22(\mathrm{~s}, 3 \mathrm{H})$;

${ }^{13} \mathrm{C}$ NMR $\left(\mathrm{CDCl}_{3}, 176 \mathrm{MHz}\right) \delta$ 170.3, $161.2(\mathrm{~d}, J=237.7 \mathrm{~Hz}), 146.3(\mathrm{~d}, J=15.0 \mathrm{~Hz}), 142.3(\mathrm{~d}, J$ $=5.1 \mathrm{~Hz}), 121.6(\mathrm{~d}, J=3.7 \mathrm{~Hz}), 117.4(\mathrm{~d}, J=31.0 \mathrm{~Hz}), 61.4,32.3,31.8$;

${ }^{19} \mathrm{~F}$ NMR $\left(\mathrm{CDCl}_{3}, 376 \mathrm{MHz}\right)-72.52$;

HRMS (LCMS ESI): calcd for $\mathrm{C}_{9} \mathrm{H}_{12} \mathrm{FN}_{2} \mathrm{O}_{2}[\mathrm{M}+\mathrm{H}]^{+}$: 199.0877. Found 199.0879.

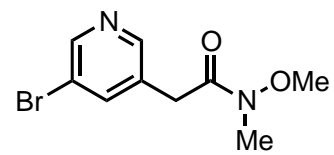

4h Prepared according to the General Procedure from the corresponding neopentyl boronic ester (202.4 mg, $0.75 \mathrm{mmol}, 1.50$ equiv.) and 3-(methoxy(methyl)amino)-3oxopropanoic acid (73.6 mg, $0.500 \mathrm{mmol}, 1.00$ equiv.) using $\mathrm{Cu}(\mathrm{OTf})_{2}(90.4 \mathrm{mg}, 0.250 \mathrm{mmol}$, 0.500 equiv.), $44 \mathrm{~h}$. Isolated in $43 \%$ yield after purification by column chromatography (Hexane/EtOAc gradient with $1 \% \mathrm{NEt}_{3}$ ) as a colorless oil.

${ }^{1} \mathrm{H}$ NMR $\left(\mathrm{CDCl}_{3}, 700 \mathrm{MHz}\right) \delta 8.57(\mathrm{~d}, J=2.1 \mathrm{~Hz}, 1 \mathrm{H}), 8.42(\mathrm{~s}, 1 \mathrm{H}), 7.82(\mathrm{~m}, 1 \mathrm{H}), 3.75(\mathrm{~s}, 2 \mathrm{H})$ $3.70(\mathrm{~s}, 3 \mathrm{H}), 3.21(\mathrm{~s}, 3 \mathrm{H})$;

${ }^{13} \mathrm{C}$ NMR $\left(\mathrm{CDCl}_{3}, 176 \mathrm{MHz}\right) \delta$ 170.7, 149.6, 148.8, 139.8, 132.4, 120.7, 61.6, 35.9, 32.5;

HRMS (LCMS ESI): calcd for $\mathrm{C}_{9} \mathrm{H}_{12} \mathrm{BrN}_{2} \mathrm{O}_{2}[\mathrm{M}+\mathrm{H}]^{+}: 259.0077$. Found 259.0079. 


\section{Additional Information, Guidelines and Troubleshooting Tips}

1. Under the standard conditions with moderately electron-rich, electron-neutral or moderately electron-poor (hetero)aromatics, neopentyl boronic esters provide superior results than pinacol boronic esters largely due to enhanced reaction rates. Aryl boron partners prone to protodeborylation can be coupled with higher yields as pinacol esters.

2. The kinetic profile of most reactions show an induction period, followed by roughly zeroorder kinetics.

3. Reactions that have a high malonate half ester to $\mathrm{Cu}$ ratio will proceed sluggishly. In cases where stoichiometry dictates an excess of the malonate half ester (ie, the aryl boron partner is the limiting reagent), increased $\mathrm{Cu}$ loadings are generally required to provide more efficient reactivity.

4. Background oxidative degradation of the malonate half esters occur slowly over a period of 2-3 days. As such, sluggish reactions will generally reach high conversions of malonate half-esters.

5. Reactions are sensitive to the reaction volume to vial size ratio. Reactions with large vial headspace or with an open cap will proceed with faster rates (high conversions in $<12 \mathrm{~h}$ ) but can result in increased side-product formation. Reactions with very little vial headspace will proceed with slower rates and require long reaction times ( $>5$ days). We attribute these observations to the importance of balancing oxygen concentration. Reactions can be set up using a dry air balloon to exclude moisture. The use of drying agents, such as molecular sieves or anhydrous metal sulfates is not recommended. Optimal reaction setups as depicted in the images below allow reactions to reach completion between $24-72 \mathrm{~h}$.

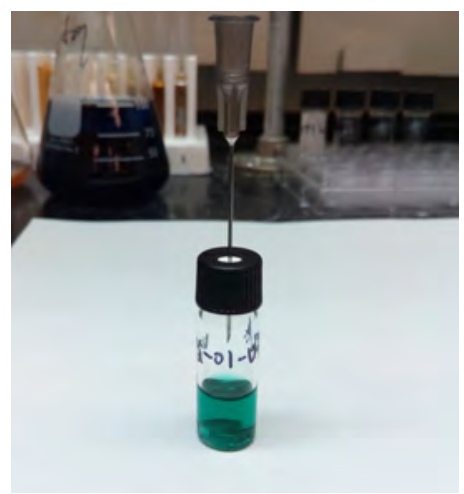

$0.2 \mathrm{mmol}$ scale 0.5 dram vial

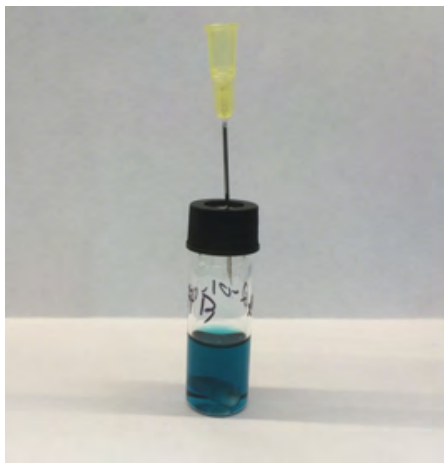

$0.5 \mathrm{mmol}$ scale 1 dram vial

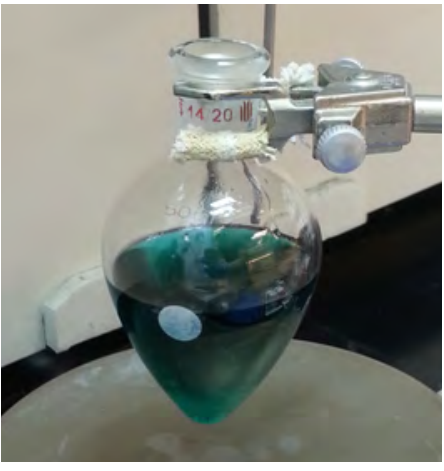

$7 \mathrm{mmol}$ scale $50 \mathrm{~mL}$ flask 
6. We have found that commercially available malonic half esters often contain more of the malonic diester than indicated. Simple basic extration followed by neutralization and reextraction provides high purity mono acid.

7. The reaction tolerates the presence of diol impurities (10-20\%) in crude aryl boronic esters, rigourous purification of the starting materials is not required.

8. 1,2-DCE can be used as the reaction solvent instead of DMA. This can be advantagous for products that are water soluble, as the DCE can be removed by rotovap and the reaction mixtures purified directly by loading onto silica gel.

9. $\mathrm{CuI}$ is good catalyst for the standard 3-iodophenyl boronic neopentyl ester substrate, but does not demonstrate broad effectiveness in comparison to $\mathrm{Cu}(\mathrm{OTf})_{2}$ across the range of substrates examined.

10. Under non-optimal conditions, oxidative degradation and decarboxylation of the substrate is observed. The volatility and/or high polarity of these products make exact quantification difficult.

11. Less successful substrates under the standard reaction conditions with mono-ethyl malonate include: 4-methoxyphenyl boronic neopentyl ester ( $29 \%$ yield with $100 \% \mathrm{Cu}$ ), 3,5dimethoxyphenyl boronic neopentyl ester ( $58 \%$ yield with $100 \% \mathrm{Cu})$, 2-(5-bromothiophenyl) boronic neopentyl ester (no product observed), 3-thiophenyl boronic neopentyl ester $(<10 \%$ product observed), 4-pyridyl boronic neopentyl ester ( $<10 \%$ product observed).

12. When the reaction is conducted under pure $\mathrm{O}_{2}$, oxidative consumption of the substrates and decarboxyation is observed. Homocoupling, aryl boron hydroxylation, and diaryl ether side products are formed under these conditions. 


\section{Arene Borylation/Decarboxylative Coupling Sequences}

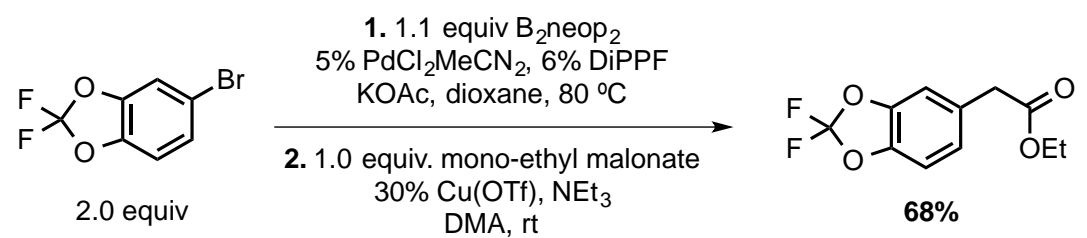

5a Step 1. In a $\mathrm{N}_{2}$-filled glovebox, $\mathrm{PdCl}_{2}\left(\mathrm{MeCN}_{2}(25.9 \mathrm{mg}, 0.100 \mathrm{mmol}, 0.0500\right.$ equiv.), 1,1'-bis(diisopropylphosphino)ferrocene ( $56.9 \mathrm{mg}, 0.120 \mathrm{mmol}, 0.0600$ equiv.), B2neop2 (497 $\mathrm{mg}, 2.20 \mathrm{mmol}, 1.10$ equiv.), KOAc (588 mg, $6.00 \mathrm{mmol}, 3.00$ equiv.) and dioxane (6.0 mL) were added to a 4-dram vial charged with a stir bar. The vial was sealed with a PTFE-lined cap and brought outside glovebox, at which point the corresponding aryl bromide (474 $\mathrm{mg}$, $2.0 \mathrm{mmol}, 1.0$ equiv.) was added. The reaction was heated at $80^{\circ} \mathrm{C}$ for $14 \mathrm{~h}$. The reaction mixture was diluted in hexanes $(5.0 \mathrm{~mL})$ and passed through a short pad of silica, washing with $\mathrm{CH}_{2} \mathrm{Cl}_{2}$, and then concentrated in vacuo to afford the corresponding crude aryl neopentyl ester which was used in the next step without further purification.

Step 2. Reaction conducted according to the General Procedure A from the corresponding crude neopentyl arylboronic ester $(1.00 \mathrm{mmol}, 2.00$ equiv., half the material from step 1$)$ and mono-ethyl malonate $\left(66.1 \mathrm{mg}, 0.500 \mathrm{mmol}, 1.00\right.$ equiv.) using $\mathrm{Cu}(\mathrm{OTf})_{2}(54.3 \mathrm{mg}, 0.150 \mathrm{mmol}$, 0.300 equiv.), $42 \mathrm{~h}$. Isolated in $65 \%$ yield after purification by column chromatography (10:1 Hexane/EtOAc) as a light yellow oil.

${ }^{1} \mathrm{H}$ NMR $\left(\mathrm{CDCl}_{3}, 500 \mathrm{MHz}\right) \delta 7.04(\mathrm{~m}, 1 \mathrm{H}), 7.00-6.97(\mathrm{~m}, 2 \mathrm{H}), 4.16(\mathrm{q}, J=7.2 \mathrm{~Hz}, 2 \mathrm{H}), 3.59$ $(\mathrm{s}, 2 \mathrm{H}), 1.26(\mathrm{t}, J=7.2 \mathrm{~Hz}, 3 \mathrm{H})$;

${ }^{13} \mathrm{C}$ NMR $\left(\mathrm{CDCl}_{3}, 125 \mathrm{MHz}\right) \delta 171.1,143.9,142.9,131.7(\mathrm{t}, J=256 \mathrm{~Hz}), 130.1,124.5,110.7$, 109.3, 61.2, 41.0, 14.2;

${ }^{19} \mathrm{~F}$ NMR $\left(\mathrm{CDCl}_{3}, 469 \mathrm{MHz}\right) \delta-50.0$;

HRMS (EI): calcd for $\mathrm{C}_{11} \mathrm{H}_{10} \mathrm{~F}_{2} \mathrm{O}_{4}[\mathrm{M}]^{+}: 244.0547$. Found 244.0551.
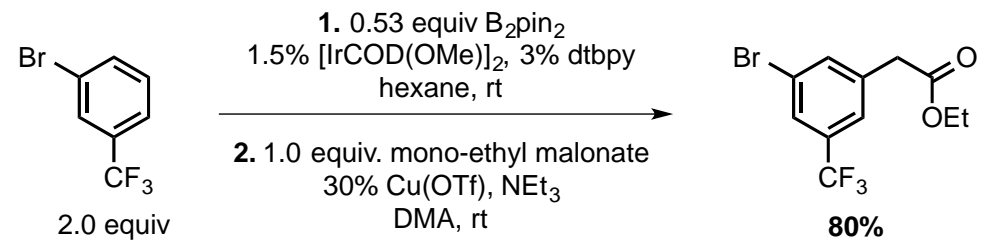

5b Step 1. In a $\mathrm{N}_{2}$-filled glovebox, $[\operatorname{Ir}(\mathrm{COD})(\mathrm{OMe})]_{2}(10.0 \mathrm{mg}, 0.0150 \mathrm{mmol}, 0.0150$ equiv.) and 4,4'-di-tert-butyl-2,2'-dipyridyl $(7.9 \mathrm{mg}, 0.030 \mathrm{mmol}, 0.030 \mathrm{mmol}, 0.030$ equiv.) were added to a 1-dram vial charged with a stir bar. Hexanes $(2.0 \mathrm{~mL})$ was added and stirred at room temperature for 10 minutes, at which point arene (225 mg, $1.00 \mathrm{mmol}, 1.00$ equiv.) and $B_{2} p_{2}$ (135 mg, $0.530 \mathrm{mmol}, 0.530$ equiv.) were sequentially added. The vial was sealed with a PTFE-lined cap and stirred at room temperature outside the glovebox. After $10 \mathrm{~h}$, the crude mixture was filtered through a plug of silica and then concentrated in vacuo to afford the corresponding crude aryl pinacol boronic ester which was used in the next step without further purification. 
Step 2. Reaction conducted according to the General Procedure A from the crude pinacol boronic ester (2.00 equiv.) and mono-ethyl malonate $(66.1 \mathrm{mg}, 0.500 \mathrm{mmol}, 1.00$ equiv.) using $\mathrm{Cu}(\mathrm{OTf})_{2}(54.3 \mathrm{mg}, 0.150 \mathrm{mmol}, 0.300$ equiv.), $18 \mathrm{~h}$. Isolated in $80 \%$ yield after purification by column chromatography (15:1 Hexane/EtOAc) as a light yellow oil.

${ }^{1} \mathrm{H} \mathrm{NMR}\left(\mathrm{CDCl}_{3}, 500 \mathrm{MHz}\right) \delta 7.68(\mathrm{~s}, 1 \mathrm{H}), 7.64(\mathrm{~s}, 1 \mathrm{H}), 7.49(\mathrm{~s}, 1 \mathrm{H}), 4.18(\mathrm{q}, J=7.1 \mathrm{~Hz}, 2 \mathrm{H})$, $3.64(\mathrm{~s}, 2 \mathrm{H}), 1.27(\mathrm{t}, J=7.2 \mathrm{~Hz}, 3 \mathrm{H})$;

${ }^{13} \mathrm{C}$ NMR $\left(\mathrm{CDCl}_{3}, 125 \mathrm{MHz}\right) \delta 170.2,137.1,135.9,132.5(\mathrm{q}, J=33 \mathrm{~Hz}), 127.3,125.0,123.1(\mathrm{q}, J$ $=274 \mathrm{~Hz}), 122.8,61.4,40.6,14.1$;

${ }^{19} \mathrm{~F} \mathrm{NMR}\left(\mathrm{CDCl}_{3}, 469 \mathrm{MHz}\right) \delta-62.8$;

HRMS (EI): calcd for $\mathrm{C}_{11} \mathrm{H}_{10} \mathrm{BrF}_{3} \mathrm{O}_{2}[\mathrm{M}]^{+}:$309.9816. Found 309.9816.

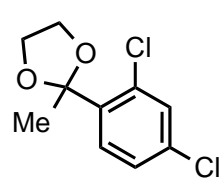

2.0 equiv.

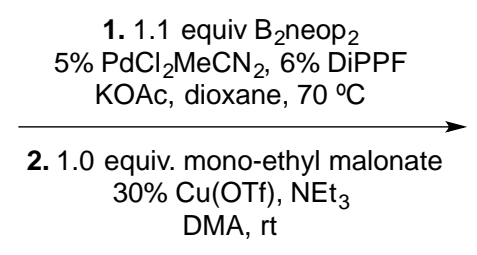

DMA, rt

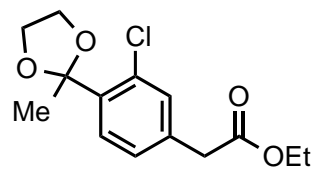

$58 \%$

5d Step 1. In a $\mathrm{N}_{2}$-filled glovebox, $\mathrm{PdCl}_{2}\left(\mathrm{MeCN}_{2}\right.$ (13 mg, $0.050 \mathrm{mmol}, 0.050$ equiv.), 1,1'bis(diisopropylphosphino)ferrocene ( $28 \mathrm{mg}, 0.060 \mathrm{mmol}, 0.060$ equiv.), and dioxane $(3.0 \mathrm{~mL})$ were added to a 4-dram vial charged with a stir bar. The corresponding aryl chloride $(233 \mathrm{mg}$, $1.00 \mathrm{mmol}, 1.00$ equiv.), B2neop 2 (270 mg, $1.20 \mathrm{mmol}, 1.20$ equiv.), and KOAc (294 mg, 3.00 mmol, 3.00 equiv.) were sequentially added. The vial was sealed with a PTFE-lined cap and heated at $70{ }^{\circ} \mathrm{C}$ outside the glovebox for $6 \mathrm{~h}$. The reaction mixture was diluted with EtOAc and washed sequentially with sat. aq. $\mathrm{NaHCO}_{3}$ and brine. The organic layer was dried with $\mathrm{Na}_{2} \mathrm{SO}_{4}$, filtered over celite and concentrated in vacuo to afford the corresponding crude neopentyl ester which was used in the next step without further purification.

Step 2. Reaction conducted according to the General Procedure A from the corresponding crude neopentyl arylboronic ester $(1.00 \mathrm{mmol}, 2.00$ equiv.) and mono-ethyl malonate (66.1 $\mathrm{mg}, 0.500 \mathrm{mmol}, 1.00$ equiv.) using $\mathrm{Cu}(\mathrm{OTf})_{2}(54.3 \mathrm{mg}, 0.150 \mathrm{mmol}, 0.300$ equiv.), $28 \mathrm{~h}$. Isolated in $58 \%$ yield after purification by column chromatography (10:1 to 2:1 pentane/Et $\mathrm{t}_{2} \mathrm{O}$ ) as a thick colorless oil.

${ }^{1} \mathrm{H}$ NMR $\left(\mathrm{CDCl}_{3}, 500 \mathrm{MHz}\right) \delta 7.58(\mathrm{~m}, 1 \mathrm{H}), 7.32(\mathrm{~m}, 1 \mathrm{H}), 7.15(\mathrm{~m}, 1 \mathrm{H}), 4.17(\mathrm{q}, J=7.1 \mathrm{~Hz}$, $2 \mathrm{H}), 4.06(\mathrm{~m}, 2 \mathrm{H}), 3.78(\mathrm{~m}, 2 \mathrm{H}), 3.57(\mathrm{~s}, 2 \mathrm{H}), 1.79(\mathrm{~s}, 3 \mathrm{H}), 1.27(\mathrm{t}, J=7.2 \mathrm{~Hz}, 3 \mathrm{H})$;

${ }^{13} \mathrm{C}$ NMR $\left(\mathrm{CDCl}_{3}, 175 \mathrm{MHz}\right) \delta$ 171.0, 138.4, 135.6, 132.2, 132.0, 127.9, 127.5, 108.4, 64.5, 61.2, 40.5, 25.3, 14.2;

HRMS (LCMS ESI): calcd for $\mathrm{C}_{14} \mathrm{H}_{17} \mathrm{ClNaO}_{4}[\mathrm{M}+\mathrm{Na}]^{+}:$307.0708. Found 307.0706. 


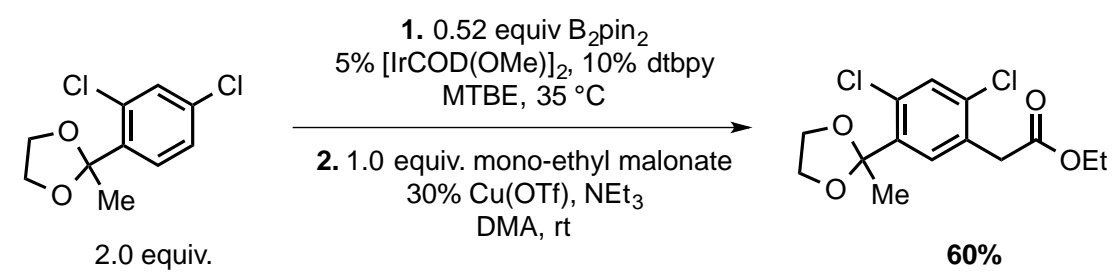

5e Step 1. In a $\mathrm{N}_{2}$-filled glovebox, $[\operatorname{Ir}(\mathrm{COD})(\mathrm{OMe})]_{2}(33.2 \mathrm{mg}, 0.0500 \mathrm{mmol}, 0.0500$ equiv.) and 4,4'-di-tert-butyl-2,2'-dipyridyl ( $26.8 \mathrm{mg}, 0.100 \mathrm{mmol}, 0.100$ equiv.) were added to a 1dram vial charged with a stir bar. MTBE $(2.0 \mathrm{~mL})$ was added and stirred at room temperature for 10 minutes, at which point arene (233 mg, $1.00 \mathrm{mmol}, 1.00$ equiv.) and $\mathrm{B}_{2}$ pinz $(132 \mathrm{mg}$, $0.520 \mathrm{mmol}, 0.520$ equiv.) were sequentially added. The vial was sealed with a PTFE-lined cap and stirred at $35^{\circ} \mathrm{C}$ outside the glovebox. After $72 \mathrm{~h}$, the crude mixture was diluted in EtOAc and sequentially washed with sat. aq. $\mathrm{NaHCO}_{3}$, sat. aq. $\mathrm{NaOAc}$, and brine. The organic layer was dried with $\mathrm{Na}_{2} \mathrm{SO}_{4}$, filtered over celite and concentrated in vacuo to afford the corresponding crude pinacol boronic ester, which was used in the next step without further purification.

Step 2. Reaction conducted according to the General Procedure A from the crude pinacol boronic ester (2.00 equiv.) and mono-ethyl malonate $(66.1 \mathrm{mg}, 0.500 \mathrm{mmol}, 1.00$ equiv.) using $\mathrm{Cu}(\mathrm{OTf})_{2}(54.3 \mathrm{mg}, 0.150 \mathrm{mmol}, 0.300$ equiv.), $24 \mathrm{~h}$. Isolated in $60 \%$ yield after purification by column chromatography (10:1 Hexane/EtOAc) as a colorless oil.

${ }^{1} \mathrm{H}$ NMR $\left(\mathrm{CDCl}_{3}, 500 \mathrm{MHz}\right) \delta 7.56(\mathrm{~s}, 1 \mathrm{H}), 7.43(\mathrm{~s}, 1 \mathrm{H}), 4.18(\mathrm{q}, J=7.0 \mathrm{~Hz}, 2 \mathrm{H}), 4.09-4.03$ $(\mathrm{m}, 2 \mathrm{H}), 3.82-3.75(\mathrm{~m}, 2 \mathrm{H}), 3.73(\mathrm{~s}, 2 \mathrm{H}), 1.77(\mathrm{~s}, 3 \mathrm{H}), 1.27(\mathrm{t}, J=7.2 \mathrm{~Hz}, 3 \mathrm{H})$;

${ }^{13} \mathrm{C}$ NMR $\left(\mathrm{CDCl}_{3}, 125 \mathrm{MHz}\right) \delta 170.2,138.5,134.6,131.8,131.5,131.0,130.5,108.1,64.5,61.2$, 38.7, 25.3, 14.2;

HRMS (LCMS ESI): calcd for $\mathrm{C}_{14} \mathrm{H}_{16} \mathrm{Cl}_{2} \mathrm{NaO}_{4}[\mathrm{M}+\mathrm{Na}]^{+}:$341.0318. Found 341.0316. 


\section{Applications in Complex Molecule Synthesis and Diversification}

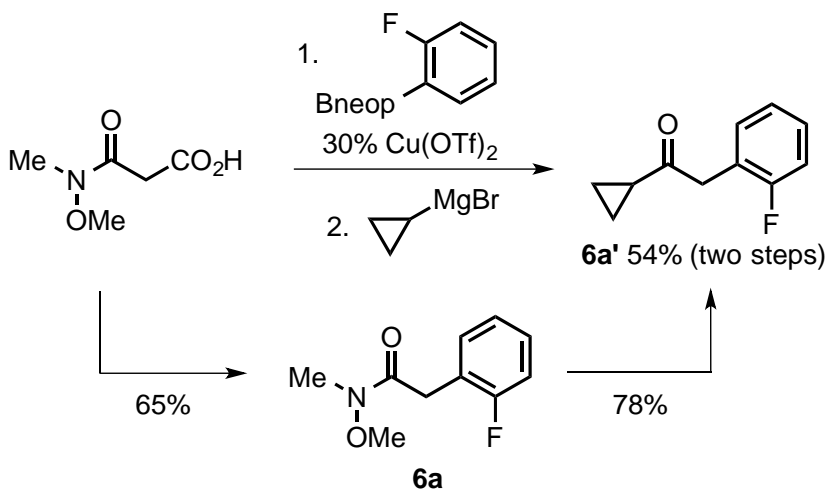

6a' Step 1 (6a). The decarboxylative coupling was conducting according to the General Procedure A from the corresponding neopentyl boronic ester (312 mg, $1.50 \mathrm{mmol}, 3.00$ equiv.) and 3-(methoxy(methyl)amino)-3-oxopropanoic acid (73.6 $\mathrm{mg}, 0.500 \mathrm{mmol}, 1.00$ equiv.) using $\mathrm{Cu}(\mathrm{OTf})_{2}(54.3 \mathrm{mg}, 0.150 \mathrm{mmol}, 0.300$ equiv.), $80 \mathrm{~h}$. Isolated in $65 \%$ yield after purification by column chromatography (3:2 Hexane/EtOAc) as a light yellow oil.

${ }^{1} \mathrm{H}$ NMR $\left(\mathrm{CDCl}_{3}, 500 \mathrm{MHz}\right) \delta 7.33-7.24(\mathrm{~m}, 2 \mathrm{H}), 7.14-7.05(\mathrm{~m}, 2 \mathrm{H}), 3.83(\mathrm{~s}, 2 \mathrm{H}), 3.70(\mathrm{~s}$, $3 \mathrm{H}), 3.23(\mathrm{~s}, 3 \mathrm{H})$;

${ }^{13} \mathrm{C}$ NMR $\left(\mathrm{CDCl}_{3}, 125 \mathrm{MHz}\right) \delta$; 171.4, $161.0(\mathrm{~d}, J=249 \mathrm{~Hz}), 131.4,128.6,124.0,122.2,115.2$, 61.2, $32.2(2 \mathrm{C})$;

${ }^{19}$ F NMR $\left(\mathrm{CDCl}_{3}, 377 \mathrm{MHz}\right) \delta-117.7$;

HRMS (LCMS ESI): calcd for $\mathrm{C}_{10} \mathrm{H}_{13} \mathrm{FNO}_{2}[\mathrm{M}+\mathrm{H}]^{+}:$198.0925. Found 198.0926.

Step 2 (6-6 $\left.\mathbf{a}^{\prime}\right)$ To a 1-dram vial sealed with a PTFE-lined cap under $\mathrm{N}_{2}$ was added cyclopropyl magnesium bromide (1.37 mL, 0.365 $\mathrm{M}$ in THF, 2.2 equiv.). The corresponding weinreb amide $(46.0 \mathrm{mg}, 0.230 \mathrm{mmol}, 1.0$ equiv.) was added as a solution in THF $(0.17 \mathrm{~mL})$, using additional THF rinces $(0.30 \mathrm{~mL})$. The solution was heated at $45^{\circ} \mathrm{C}$ for 1 hour. The mixture was cooled to $0^{\circ} \mathrm{C}$ and quenched with $1 \mathrm{M} \mathrm{HCl}$ solution $(1.0 \mathrm{~mL})$. Additional water (5 $\mathrm{mL})$ was added and then the reaction was extracted with EtOAc $(3 \times 10 \mathrm{~mL}$ EtOAc). The organic layer was washed with brine $(5 \mathrm{~mL})$ and water $(5 \mathrm{~mL})$, then dried with $\mathrm{Na}_{2} \mathrm{SO}_{4}$, and concentrated in vacuo. The crude mixture was passed through a plug of silica, washing with 10:1 hexane/EtOAc, then concentrated to afford the title compound (78\% yield) as a light yellow oil. Spectroscopic data agreed with that reported. ${ }^{17}$

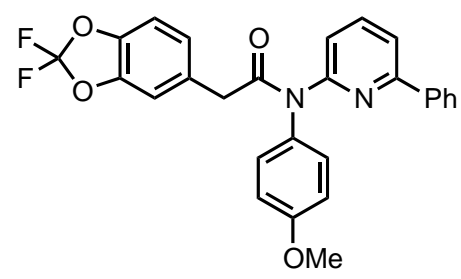

6b Prepared according to the General Procedure A from the corresponding neopentyl boronic ester (108 mg, $0.400 \mathrm{mmol}, 2.00$ equiv.) and 3-((4-methoxyphenyl)(6-phenylpyridin-2yl)amino)-3-oxopropanoic acid ( $85.3 \mathrm{mg}, 0.200 \mathrm{mmol}, 1.00$ equiv., $85 \%$ pure $(\mathrm{w} / \mathrm{w}))$ using 
$\mathrm{Cu}(\mathrm{OTf})_{2}(36.3 \mathrm{mg}, 0.100 \mathrm{mmol}, 0.500$ equiv.), $44 \mathrm{~h}$. Isolated in $74 \%$ yield after purification by column chromatography (65:35 Hexane/EtOAc) as a light yellow oil.

${ }^{1} \mathbf{H}$ NMR $\left(\mathrm{CDCl}_{3}, 500 \mathrm{MHz}\right) \delta 7.90-7.87(\mathrm{~m}, 2 \mathrm{H}), 7.71(\mathrm{~m}, 1 \mathrm{H}), 7.59(\mathrm{~m}, 1 \mathrm{H}), 7.45-7.37(\mathrm{~m}$, $3 \mathrm{H}), 7.30-7.20(\mathrm{~m}, 3 \mathrm{H}), 6.95-6.90(\mathrm{~m}, 4 \mathrm{H}), 6.80(\mathrm{~m}, 1 \mathrm{H}), 3.84(\mathrm{~s}, 3 \mathrm{H}), 3.78(\mathrm{~s}, 2 \mathrm{H})$;

${ }^{13} \mathrm{C}$ NMR $\left(\mathrm{CDCl}_{3}, 125 \mathrm{MHz}\right) \delta 171.4,159.0,156.1,154.8,143.7,142.6,138.6,138.2,134.3$, $131.6(t, J=254 \mathrm{~Hz}), 131.2,129.8,129.3,128.7,126.7,124.4,119.0,117.6,114.5,110.7,109.0,55.5$, 42.2;

${ }^{19}$ F NMR $\left(\mathrm{CDCl}_{3}, 469 \mathrm{MHz}\right) \delta-49.9$;

HRMS (LCMS ESI): calcd for $\mathrm{C}_{27} \mathrm{H}_{20} \mathrm{~F}_{2} \mathrm{~N}_{2} \mathrm{NaO}_{4}[\mathrm{M}+\mathrm{Na}]^{+}:$497.1283. Found 497.1294.
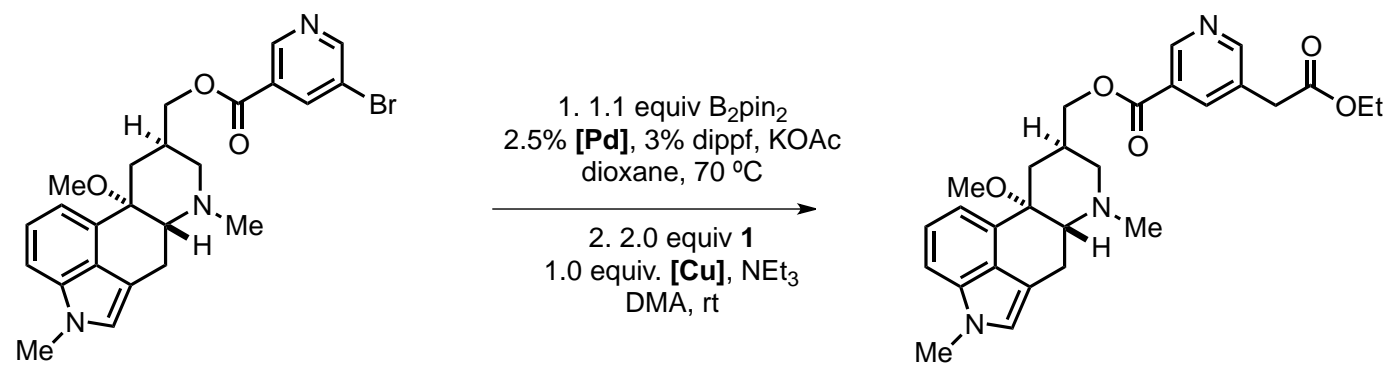

7a In a $\mathrm{N}_{2}$-filled glovebox, $\mathrm{PdCl}_{2}\left(\mathrm{MeCN}_{2}(3.6 \mathrm{mg}, 0.014 \mathrm{mmol}, 0.025\right.$ equiv. $), 1,1^{\prime}$ Bis(diisopropylphosphino)ferrocene ( $7.0 \mathrm{mg}, 0.017 \mathrm{mmol}, 0.030$ equiv.), and dioxane (1.5 $\mathrm{mL}$ ) were added to a 1-dram vial charged with a stir bar. The corresponding aryl bromide (271 $\mathrm{mg}, 0.560 \mathrm{mmol}, 1.00$ equiv.), B2pinz (156 mg, $0.620 \mathrm{mmol}, 1.10$ equiv.), and KOAc (165 mg, $1.68 \mathrm{mmol}, 3.00$ equiv.) were sequentially added. The vial was sealed with a PTFE-lined cap and heated at $70^{\circ} \mathrm{C}$ outside the glovebox for $4 \mathrm{~h}$. The reaction mixture was passed through a plug of celite, washing with toluene $(50 \mathrm{~mL})$. The organic layer was sequentially washed with sat. $\mathrm{NaHCO}_{3}(20 \mathrm{~mL})$ and brine $(3 \times 20 \mathrm{~mL})$, and then dried with $\mathrm{NaSO}_{4}$. The solvent was removed in vacuo to afford the corresponding pinacol arylboronic ester as a pale brown solid, $97 \%$ yield.

Step 2. Prepared according to the General Procedure A from the corresponding pinacol arylboronic ester $(66.4 \mathrm{mg}, 0.125 \mathrm{mmol}, 1.00$ equiv.) and mono-ethyl malonate ( $33.0 \mathrm{mg}, 0.250$ mmol, 2.00 equiv.) using $\mathrm{Cu}(\mathrm{OTf})_{2}\left(45.2 \mathrm{mg}, 0.125 \mathrm{mmol}, 1.00\right.$ equiv.) and $\mathrm{Et}_{3} \mathrm{~N}(0.17 \mathrm{~mL}, 1.25$ mmol, 10 equiv.), $25 \mathrm{~h}$. Isolated in $57 \%$ yield after purification by column chromatography ( $4 \%$ to $6 \% \mathrm{MeOH}$ in $\mathrm{CH}_{2} \mathrm{Cl}_{2} / \mathrm{MeCN} / \mathrm{Hexanes}$ (2:1:1), $1 \% \mathrm{NH}_{4} \mathrm{Cl}$ additive) as a colorless oil.

${ }^{1} \mathrm{H} \mathrm{NMR}\left(\mathrm{CDCl}_{3}, 500 \mathrm{MHz}\right) \delta 9.15(\mathrm{~s}, 1 \mathrm{H}), 8.70(\mathrm{~s}, 1 \mathrm{H}), 8.25(\mathrm{~m}, 1 \mathrm{H}), 7.24(\mathrm{~m}, 1 \mathrm{H}), 7.20(\mathrm{~m}$, $1 \mathrm{H}), 7.05(\mathrm{~m}, 1 \mathrm{H}), 6.80(\mathrm{~s}, 1 \mathrm{H}), 4.40(\mathrm{~m}, 1 \mathrm{H}), 4.30(\mathrm{~m}, 1 \mathrm{H}), 4.18(\mathrm{q}, J=7.1 \mathrm{~Hz}, 2 \mathrm{H}), 3.78(\mathrm{~s}, 3 \mathrm{H})$, $3.69(\mathrm{~s}, 2 \mathrm{H}), 3.26-3.19(\mathrm{~m}, 2 \mathrm{H}), 3.06-2.96(\mathrm{~m}, 5 \mathrm{H}), 2.65(\mathrm{br}, 1 \mathrm{H}), 2.49(\mathrm{~s}, 3 \mathrm{H}), 2.39(\mathrm{br}, 1 \mathrm{H})$, $2.13(\mathrm{br}, 1 \mathrm{H}), 1.39(\mathrm{t}, J=13 \mathrm{~Hz}, 1 \mathrm{H}), 1.26(\mathrm{t}, J=7.2 \mathrm{~Hz}, 3 \mathrm{H})$;

${ }^{13} \mathrm{C}$ NMR $\left(\mathrm{CDCl}_{3}, 125 \mathrm{MHz}\right) \delta 170.2,165.1,154.2,149.6,137.9,135.2,130.0,129.6,126.3$, $125.9,123.3,121.5,115.0,110.2,109.0,73.6,70.1,68.1,61.5,60.6,49.6,43.9,38.2,32.8,31.5,30.2$, 22.3, 14.2;

HRMS (LCMS ESI): calcd for $\mathrm{C}_{28} \mathrm{H}_{34} \mathrm{~N}_{3} \mathrm{O}_{5}[\mathrm{M}+\mathrm{H}]^{+}: 492.2493$. Found 492.2494. 

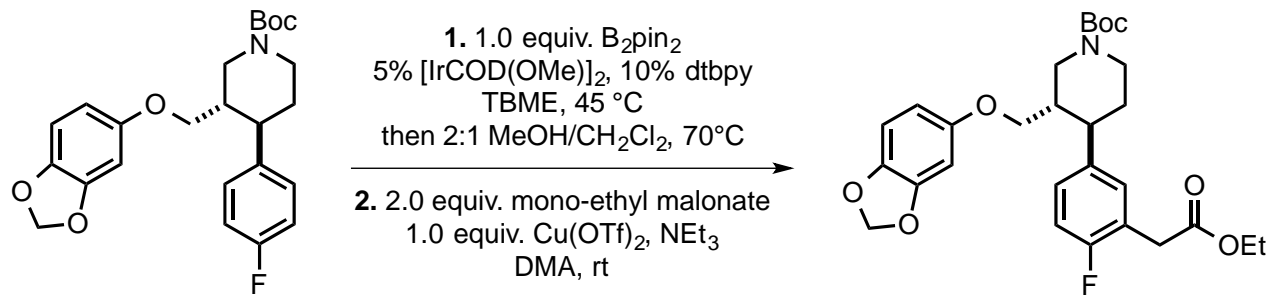

7b Step 1. In a $\mathrm{N}_{2}$-filled glovebox, [ $\left.\operatorname{Ir}(\mathrm{COD})(\mathrm{OMe})\right]_{2}(15 \mathrm{mg}, 0.023 \mathrm{mmol}, 0.050$ equiv.) and 4,4'-di-tert-butyl-2,2'-dipyridyl (12 mg, $0.045 \mathrm{mmol}, 0.10$ equiv.) were added to a 1-dram vial charged with a stir bar. MTBE $(1.5 \mathrm{~mL})$ was added and stirred at room temperature for 10 minutes, at which point arene (195 mg, $0.450 \mathrm{mmol}, 1.00$ equiv.) and B2pinz (114 mg, 0.450 mmol, 1.0 equiv.) were sequentially added. The vial was sealed with a PTFE-lined cap and stirred at $45{ }^{\circ} \mathrm{C}$ outside the glovebox. After $16 \mathrm{~h}$, the crude mixture was concentrated,

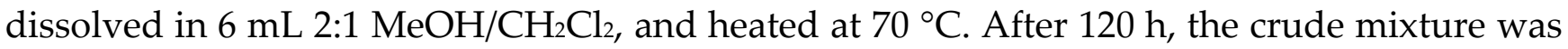
concentrated. Isolated in $22 \%$ yield ( $38 \%$ yield based on recovered arene) after purification by column chromatography (10:1 to 4:1 Hexane/EtOAc) as a white solid.

Step 2. Prepared according to the General Procedure A from the corresponding pinacol arylboronic ester ( $48 \mathrm{mg}, 0.086 \mathrm{mmol}, 1.00$ equiv.) and mono-ethyl malonate ( $23 \mathrm{mg}, 0.17$ mmol, 2.00 equiv.) using $\mathrm{Cu}(\mathrm{OTf})_{2}\left(31 \mathrm{mg}, 0.086 \mathrm{mmol}, 1.00\right.$ equiv.) and $\mathrm{Et}_{3} \mathrm{~N}(0.12 \mathrm{~mL}, 0.86$ mmol, 10 equiv.), $23 \mathrm{~h}$. Isolated in $45 \%$ yield (76\% yield based on recovered arene) after purification by column chromatography (10:1 to 4:1 Hexane/EtOAc) as a colorless oil.

${ }^{1} \mathrm{H}$ NMR $\left(\mathrm{CDCl}_{3}, 500 \mathrm{MHz}, 65^{\circ} \mathrm{C}\right) \delta 7.09-7.04(\mathrm{~m}, 2 \mathrm{H}), 6.97(\mathrm{t}, J=9.2 \mathrm{~Hz}, 1 \mathrm{H}), 6.61(\mathrm{~d}, J=$ $8.6 \mathrm{~Hz}, 1 \mathrm{H}), 6.35(\mathrm{~m}, 1 \mathrm{H}), 6.16(\mathrm{~m}, 1 \mathrm{H}), 5.86(\mathrm{~s}, 2 \mathrm{H}), 4.43(\mathrm{~m}, 1 \mathrm{H}), 4.24(\mathrm{~m}, 1 \mathrm{H}), 4.15(\mathrm{q}, J=7.1$ $\mathrm{Hz}, 2 \mathrm{H}), 3.64(\mathrm{~m}, 1 \mathrm{H}), 3.60(\mathrm{~s}, 2 \mathrm{H}), 3.49(\mathrm{~m}, 1 \mathrm{H}), 2.84-2.76(\mathrm{~m}, 2 \mathrm{H}), 2.65(\mathrm{~m}, 1 \mathrm{H}), 2.01(\mathrm{~m}, 1 \mathrm{H})$, $1.81(\mathrm{~m}, 1 \mathrm{H}), 1.71(\mathrm{~m}, 1 \mathrm{H}), 1.50(\mathrm{~s}, 9 \mathrm{H}), 1.23(\mathrm{t}, J=7.1 \mathrm{~Hz}, 3 \mathrm{H})$;

${ }^{13} \mathrm{C}$ NMR $\left(\mathrm{CDCl}_{3}, 500 \mathrm{MHz}, 65{ }^{\circ} \mathrm{C}\right) \delta 170.5,160.0(\mathrm{~d}, J=244 \mathrm{~Hz}), 154.9,154.6,148.4,142.0$, $139.4(\mathrm{~d}, J=3.6 \mathrm{~Hz}), 130.5(\mathrm{~d}, J=4.1 \mathrm{~Hz}), 127.8(\mathrm{~d}, J=8.1 \mathrm{~Hz}), 121.9$ (d, $J=16 \mathrm{~Hz}), 115.5(\mathrm{~d}, J=$ $22 \mathrm{~Hz}), 108.0,106.3,101.2,98.4,79.7,69.4,61.0,47.5,44.5,44.4,42.1,34.6$ (d, $J=2.9 \mathrm{~Hz}), 34.1$, 28.6, 14.2;

${ }^{19} \mathrm{~F}$ NMR $\left(\mathrm{CDCl}_{3}, 468 \mathrm{MHz}, 65^{\circ} \mathrm{C}\right) \delta-120.3$;

HRMS (LCMS ESI): calcd for $\mathrm{C}_{28} \mathrm{H}_{34} \mathrm{FNNaO}_{7}[\mathrm{M}+\mathrm{Na}]^{+}$: 538.2212. Found 538.2212.
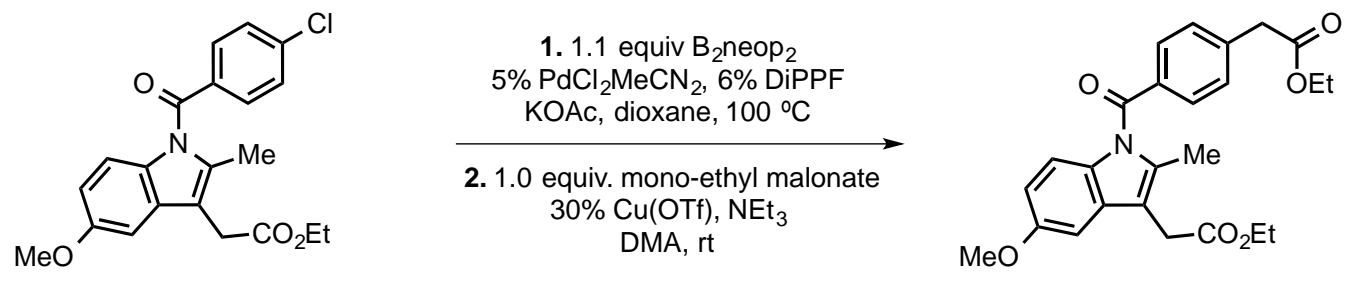

7b $63 \%$

7c Step 1. In a $\mathrm{N}_{2}$-filled glovebox, $\mathrm{PdCl}_{2}\left(\mathrm{MeCN}_{2}\right.$ (13 mg, $0.050 \mathrm{mmol}, 0.050$ equiv.), 1, 1'bis(diisopropylphosphino)ferrocene $(28 \mathrm{mg}, 0.060 \mathrm{mmol}, 0.060$ equiv.), and dioxane $(3.0 \mathrm{~mL}$ ) were added to a 4-dram vial charged with a stir bar. Indometacin ethyl ester (386 mg, 1.00 mmol, 1.00 equiv.), B2neop 2 (249 mg, $1.10 \mathrm{mmol}, 1.10$ equiv.), and KOAc (294 mg, $3.00 \mathrm{mmol}$, 3.00 equiv.) were sequentially added. The vial was sealed with a PTFE-lined cap and heated 
at $100^{\circ} \mathrm{C}$ outside the glovebox for $2.5 \mathrm{~h}$. The reaction mixture was diluted with EtOAc and washed sequentially with sat. aq. $\mathrm{NaHCO}_{3}$ and brine. The organic layer was dried with $\mathrm{Na}_{2} \mathrm{SO}_{4}$, filtered over celite and concentrated in vacuo. Azeoptropical removal of trace water with toluene afforded the corresponding crude aryl neopentyl ester which was used in the next step without further purification.

Step 2. The reaction was conducted according to the General Procedure A from the corresponding crude neopentyl boronic ester (1.00 mmol, 2.00 equiv.) and mono-ethyl malonate (66.1 mg, $0.500 \mathrm{mmol}, 1.00$ equiv.) using $\mathrm{Cu}(\mathrm{OTf})_{2}(54.3 \mathrm{mg}, 0.150 \mathrm{mmol}, 0.300$ equiv.), $68 \mathrm{~h}$. Isolated in $63 \%$ yield after purification by column chromatography (7:3 Hexane/EtOAc) as an off-white solid.

${ }^{1} \mathrm{H}$ NMR $\left(\mathrm{CDCl}_{3}, 500 \mathrm{MHz}\right) \delta 7.69-7.67(\mathrm{~m}, 2 \mathrm{H}), 7.41-7.39(\mathrm{~m}, 2 \mathrm{H}), 6.97(\mathrm{~m}, 1 \mathrm{H}), 6.90(\mathrm{~m}$, $1 \mathrm{H}), 6.65(\mathrm{~m}, 1 \mathrm{H}), 4.21-4.14(\mathrm{~m}, 4 \mathrm{H}), 3.84(\mathrm{~s}, 3 \mathrm{H}), 3.71(\mathrm{~s}, 2 \mathrm{H}), 3.65(\mathrm{~s}, 2 \mathrm{H}), 2.37(\mathrm{~s}, 3 \mathrm{H}), 1.28-$ $1.24(\mathrm{~m}, 6 \mathrm{H})$;

${ }^{13} \mathrm{C}$ NMR $\left(\mathrm{CDCl}_{3}, 125 \mathrm{MHz}\right) \delta 171.0,170.7,169.2,156.0,139.4,136.0,134.4,131.0,130.6$, 130.1, 129.7, 115.1, 112.4, 111.6, 101.2, 61.2, 61.0, 55.8, 41.5, 30.5, 14.3, 14.2, 13.4;

HRMS (LCMS ESI): calcd for $\mathrm{C}_{25} \mathrm{H}_{27} \mathrm{NNaO}_{6}[\mathrm{M}+\mathrm{Na}]^{+}: 460.1731$. Found 460.1731 .
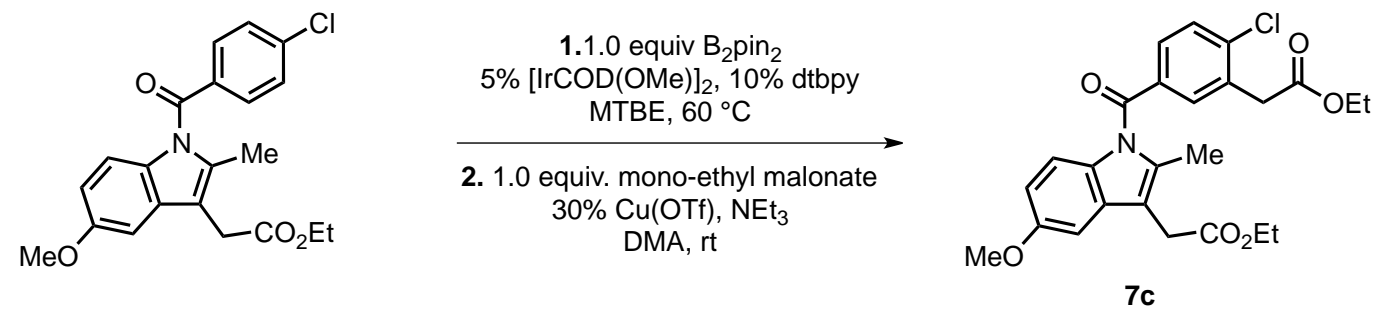

7c Step 1. In a N2-filled glovebox, $[\mathrm{Ir}(\mathrm{COD})(\mathrm{OMe})]_{2}(9.9 \mathrm{mg}, 0.015 \mathrm{mmol}, 0.050$ equiv.) and 4,4'-Di-tert-butyl-2,2'-dipyridyl $(8.0 \mathrm{mg}, 0.030 \mathrm{mmol}, 0.10$ equiv.) were added to a 1-dram vial charged with a stir bar. MTBE $(1.0 \mathrm{~mL})$ was added and stirred at room temperature for 10 minutes, at which point arene (116 mg, $0.300 \mathrm{mmol}, 1.00$ equiv.) and $\mathrm{B}_{2}$ pin 2 (76.2 mg, 0.300 mmol, 1.0 equiv.) were sequentially added. The vial was sealed with a PTFE-lined cap and stirred at $60^{\circ} \mathrm{C}$ outside the glovebox. After $4 \mathrm{~h}$, the crude mixture was concentrated and passed through a short silica column (4:1 to 1:1 Hexane/EtOAc). Isolated in $43 \%$ yield as a pale yellow oil.

Step 2. The reaction was conducting according to the General Procedure A from the corresponding pinacol arylboronic ester (123 mg, $0.24 \mathrm{mmol}, 2.00$ equiv.) and mono-ethyl malonate (15.9 mg, $0.120 \mathrm{mmol}, 1.00$ equiv.) using $\mathrm{Cu}(\mathrm{OTf}) 2(13.0 \mathrm{mg}, 0.0360 \mathrm{mmol}, 0.300$ equiv.), $40 \mathrm{~h}$. Isolated in 53\% yield after purification by column chromatography (4:1 to 2:1 Hexane/EtOAc) as a light yellow oil.

${ }^{1} \mathrm{H}$ NMR $\left(\mathrm{CDCl}_{3}, 500 \mathrm{MHz}\right) \delta 7.67(\mathrm{~m}, 1 \mathrm{H}), 7.61(\mathrm{~m}, 1 \mathrm{H}), 7.53(\mathrm{~m}, 1 \mathrm{H}), 6.99(\mathrm{~m}, 1 \mathrm{H}), 6.96$ $(\mathrm{m}, 1 \mathrm{H}), 6.70(\mathrm{~m}, 1 \mathrm{H}), 4.21-4.16(\mathrm{~m}, 4 \mathrm{H}), 3.86(\mathrm{~s}, 3 \mathrm{H}), 3.82(\mathrm{~s}, 2 \mathrm{H}), 3.67(\mathrm{~s}, 2 \mathrm{H}), 2.39(\mathrm{~s}, 3 \mathrm{H})$, $1.30-1.25(\mathrm{~m}, 6 \mathrm{H})$;

${ }^{13} \mathrm{C}$ NMR $\left(\mathrm{CDCl}_{3}, 125 \mathrm{MHz}\right) \delta 170.9,169.8,168.2,156.1,139.5,135.9,134.3,133.6,132.8$, 130.9, 130.7, 130.0, 129.9, 115.1, 112.8, 111.8, 101.3, 61.3, 61.0, 55.7, 39.1, 30.5, 14.3, 14.2, 13.4;

HRMS (LCMS ESI): calcd for $\mathrm{C}_{25} \mathrm{H}_{26} \mathrm{ClNNaO}_{6}[\mathrm{M}+\mathrm{Na}]^{+}: 494.1341$. Found 494.1345 


\section{References}

1. Still, W.C.; Kahn, M.; Mitra, A., J. Org. Chem. 1978, 43, 2923-2925.

2. Matthew, S. C.; Glasspoole, B. W.; Eisenberger, P.; Crudden, C. M., J. Am. Chem. Soc. 2014, $136,5828-5831$.

3. Huang, Y-J.; Jiang, Y-B.; Bull, S. D.; Fossey, J. S.; James T. D., Chem. Commun. 2010, 46, 81808182.

4. Yin, G.; Kalvet, I.; Englert, U.; Schoenebeck, F., J. Am. Chem. Soc. 2015, 137, 4164-4172.

5. Thietiot-Laurent, S. A. L.; Nadal, B.; Le Gall, T., Synthesis 2010, 10, 1697-1701.

6. Cordes, J.; Calo, F.; Anderson, K.; Pfaffeneder, T.; Laclef, S.; White, A. J. P.; Barrett, A. G. M., J. Org. Chem. 2012, 77, 652-657.

7. Chen, Y.; Sieburth, S. McN., Synthesis 2002, 15, 2191-2194.

8. Ryu, Y.; Scott, A. I., Tetrahedron Lett. 2003, 44, 7499-7502.

9. Marom, E.; Mizhiritskii, M.; Rubnov, S., Intermediate Compounds and Processes for the Preperation of Quinoline Derivatives such as Laquinimob Sodium. WO 2012070051 A1, May 31, 2012.

10. Patel, D. V.; Young, M. G.; Robinson, S. P.; Hunihan, L.; Dean, B. J.; Gordon, E. M., J. Med. Chem. 1996, 39, 4197-4210.

11. Redman-Furey, N. L.; Godlewski, J. E.; Dicks, M. L., Malate salts, and polymorphs of (3S,5S)-7-[3-amino-5-methyl-piperidinyl]-1-cyclopropyl-1,4-dihydro-8-methoxy-4-oxo-3quinolinecarboxylic acid. US Patent 232650 A1, October 4, 2007.

12. Ramaprasad, G. C.; Kalluraya, B.; Kumar, B. S.; Hunnur, R. K., Eur. J. Med. Chem. 2010, 45, 4587-4593.

13. Xie, P.; Xie, P. Y.; Qian, B.; Zhou, H.; Xia, C.; Huang, H., J. Am. Chem. Soc. 2012, 134, $9902-$ 9905.

14. Feng, Y.; W. Wu, W.; Xu, Z.; Li, Y.; Li, M.; Xu, H., Tetrahedron 2012, 68, 2113-2120.

15. Zimmermann, B.; Dzik, W. I.; Himmler, T.; Goossen, L. J., J. Org. Chem. 2011, 76, 8107-8112.

16. Penga, Z.; Wang, J.; Cheng, J.; Xie, X.; Zhang, Z., Tetrahedron 2010, 66, 8238-8241.

17. Manne, S.; Srinivasan, T.; Sajja, E.; Karamala, R.; Bairy, K.; Ghojala, V., Processes for Preparing Prasugrel and Pharmaceutically Acceptable Salts Thereof. US Patent 2012202066 A1, August 9, 2012. 
XI. NMR Spectra

Department of Chemistry, University of Alberta

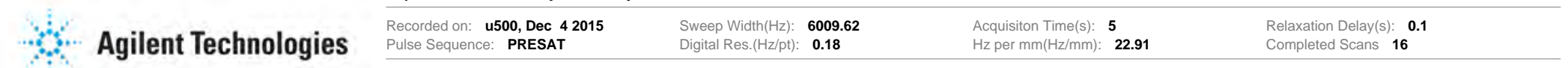

\section{Rylan, RL-05-63}

499.806 MHz H1 PRESAT in cdcl3 (ref. to CDCl3 @ 7.26 ppm), temp $27.7 \mathrm{C}$-> actual temp $=27.0 \mathrm{C}$, colddual probe

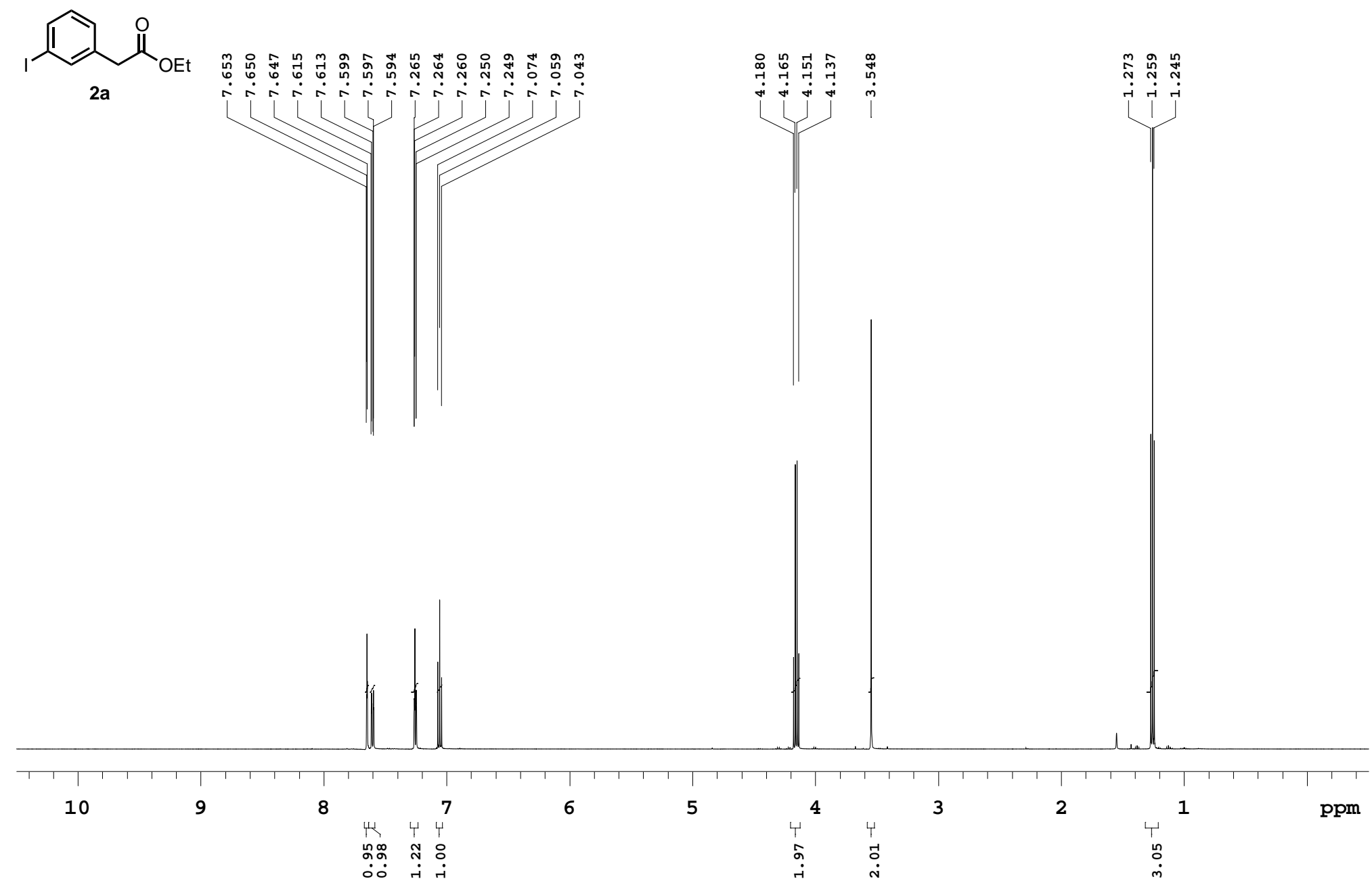

File: /mnt/d600/home13/rilnmr/nmrdata/DATA_FROM_NMRSERVICE/Rylan/2015.12/2015.12.04.u5_RL-05-63_loc6_04.06_H1_1D 
Department of Chemistry, University of Alberta

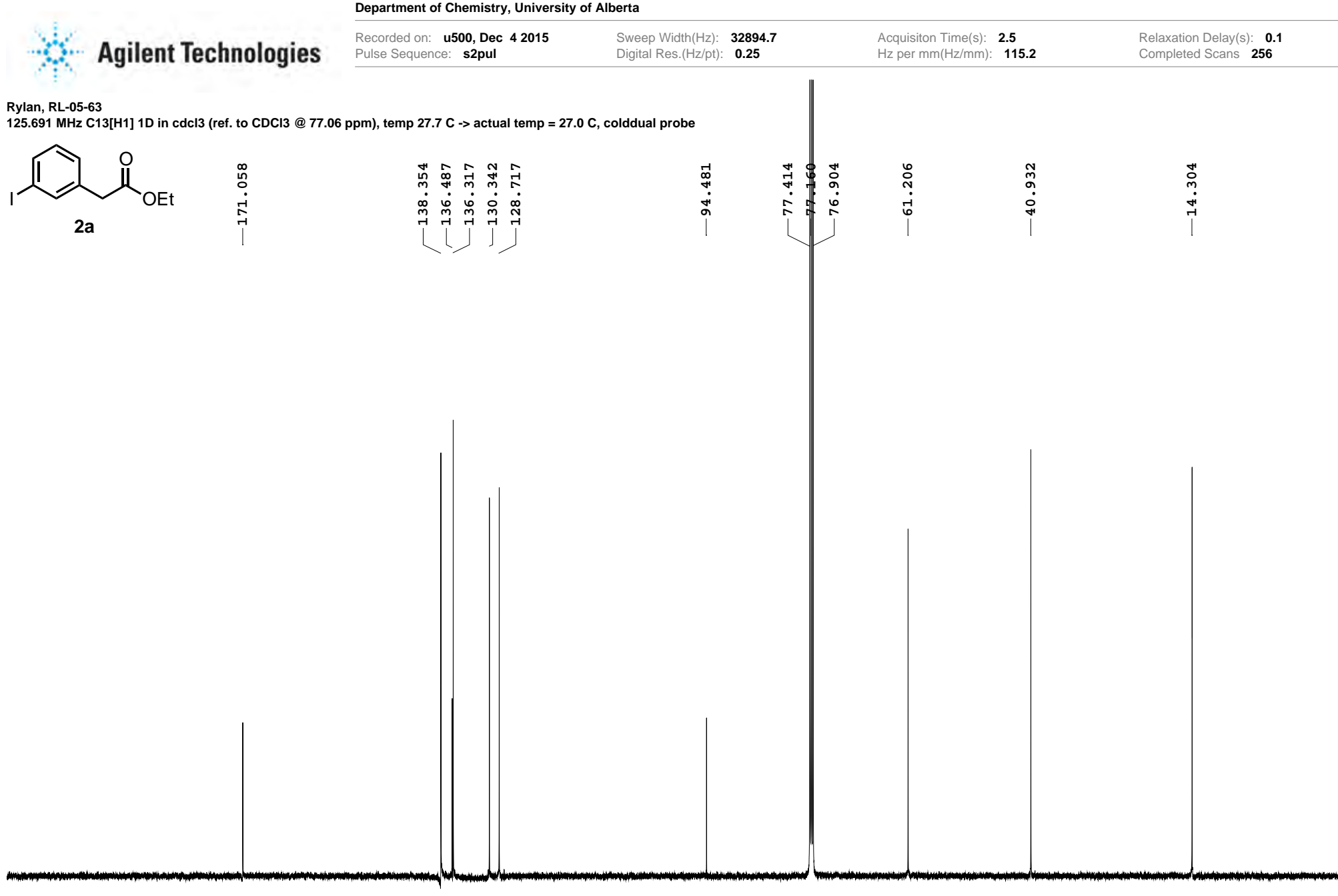
200
180
160
140
120
100
80
60
40
20
ppm 
Department of Chemistry, University of Alberta
Agilent Technologies
Biscondied on v700, May 112016 Sweep Wounctis 8389.2 Aopusion Trieis 5 32.07

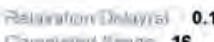
Shengkang, SY-04-23

698.762 MHz H1 PRESAT in odel3 (ret. to CDC13 @ $7.26 \mathrm{ppm}$ ), temp $27.5 \mathrm{C} \rightarrow$ actual temp $=27.0 \mathrm{C}$, coldid probe

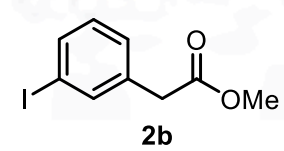

2b

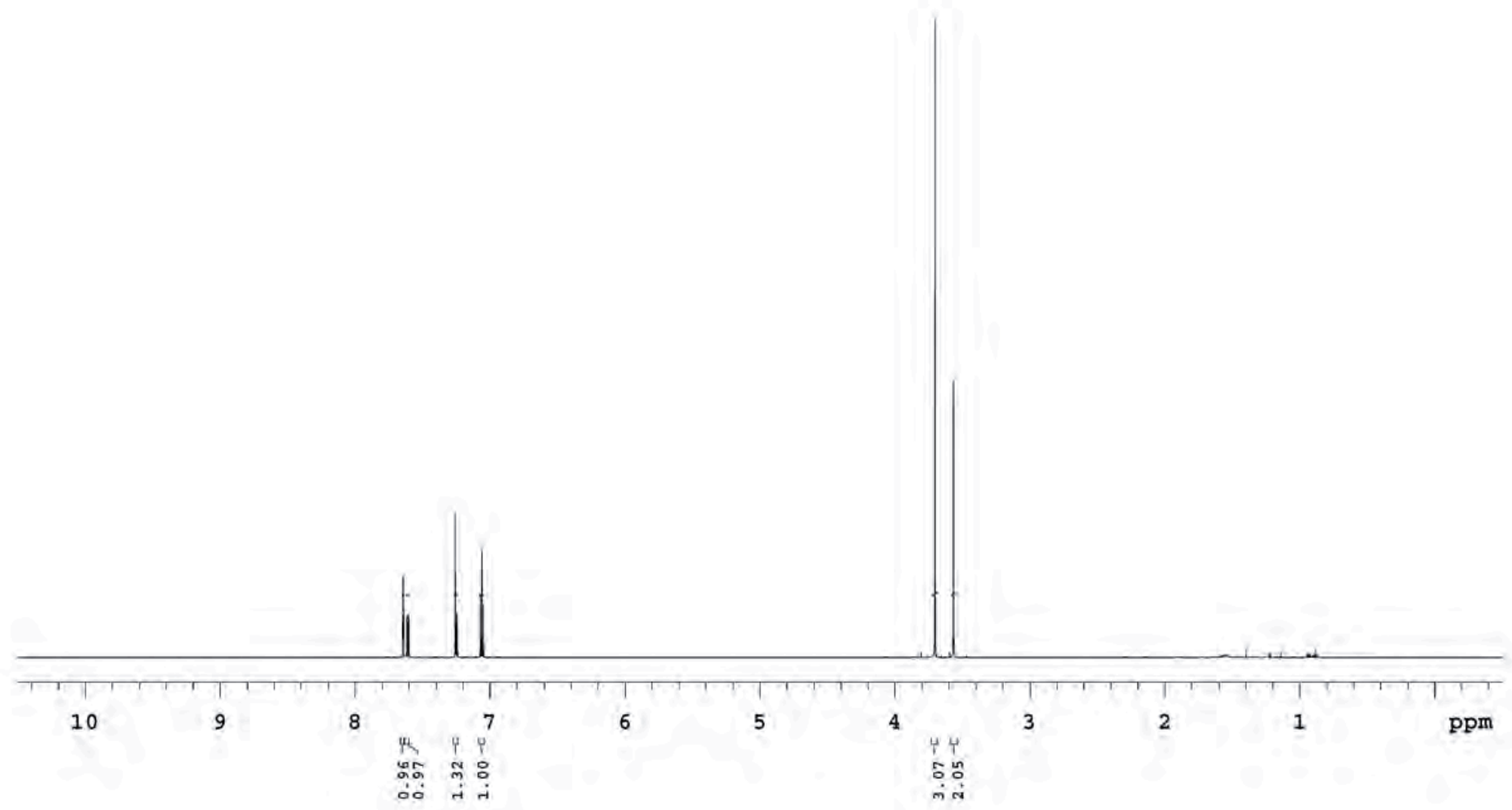


Department of Chemistry, University of Alberta

\begin{tabular}{|c|c|c|c|c|}
\hline Agilent Technologies & 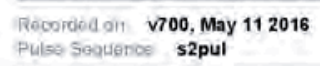 & 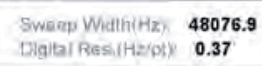 & 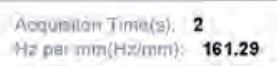 & 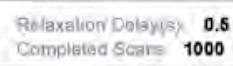 \\
\hline
\end{tabular}
Shengkang, SY-04-23-DRY $175.975 \mathrm{MHz} \mathrm{C} 13[\mathrm{H} 1]$ ] 10 in odel3 (ret. to $\mathrm{CDC} 13$ (3) $77.06 \mathrm{ppm}$ ), temp $27.5 \mathrm{C} \rightarrow$ actual temp $=27.0 \mathrm{C}$, coldid probe
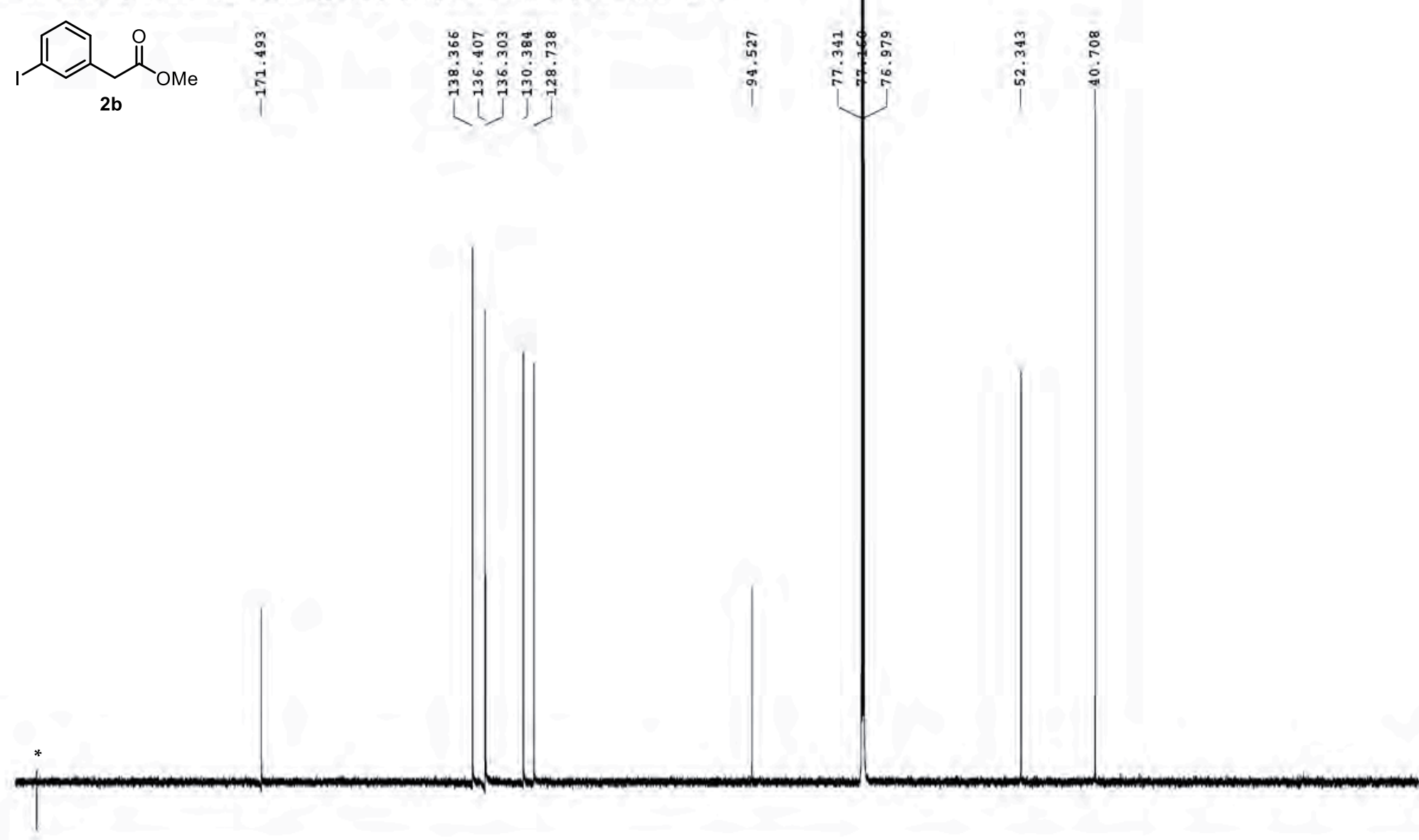

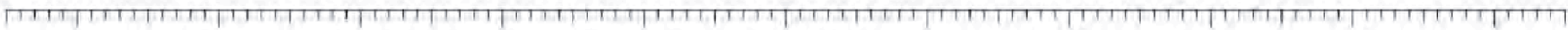


Department of Chemistry, University of Alberta

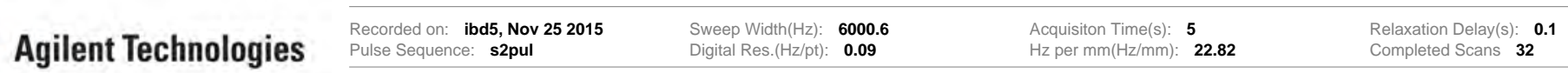

PM-06-039-B-F11-14

498.118 MHz H1 1D in cdcl3 (ref. to CDCI3 @ 7.26 ppm), temp 26.4 C -> actual temp = 27.0 C, autoxdb probe

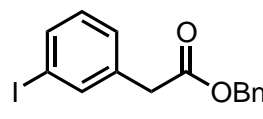

2c

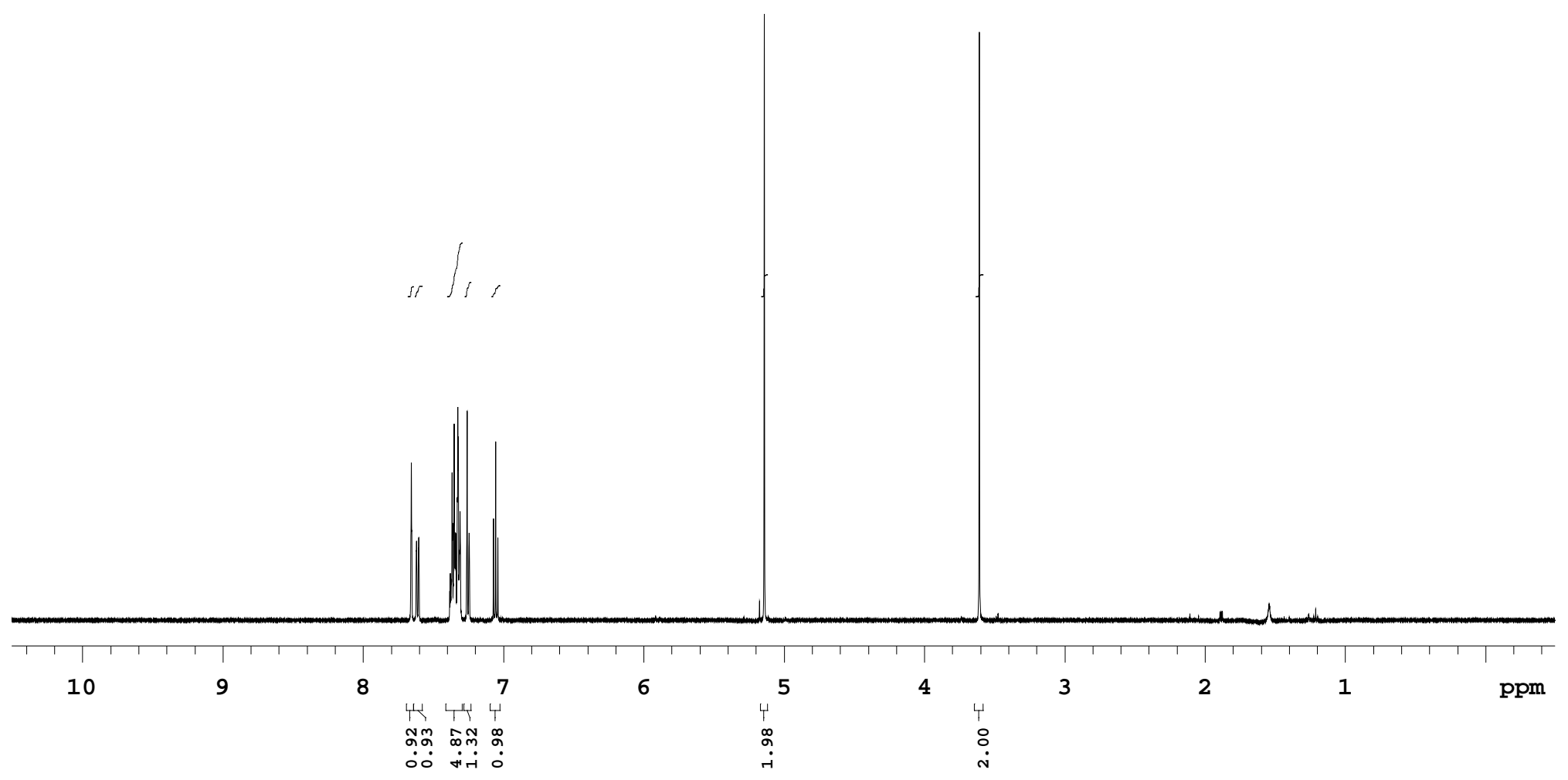

File: /mnt/d600/home13/rilnmr/nmrdata/Patrick/2015.11/2015.11.25.15_PM-06-039-B-F11-14_H1_1D 
Department of Chemistry, University of Alberta

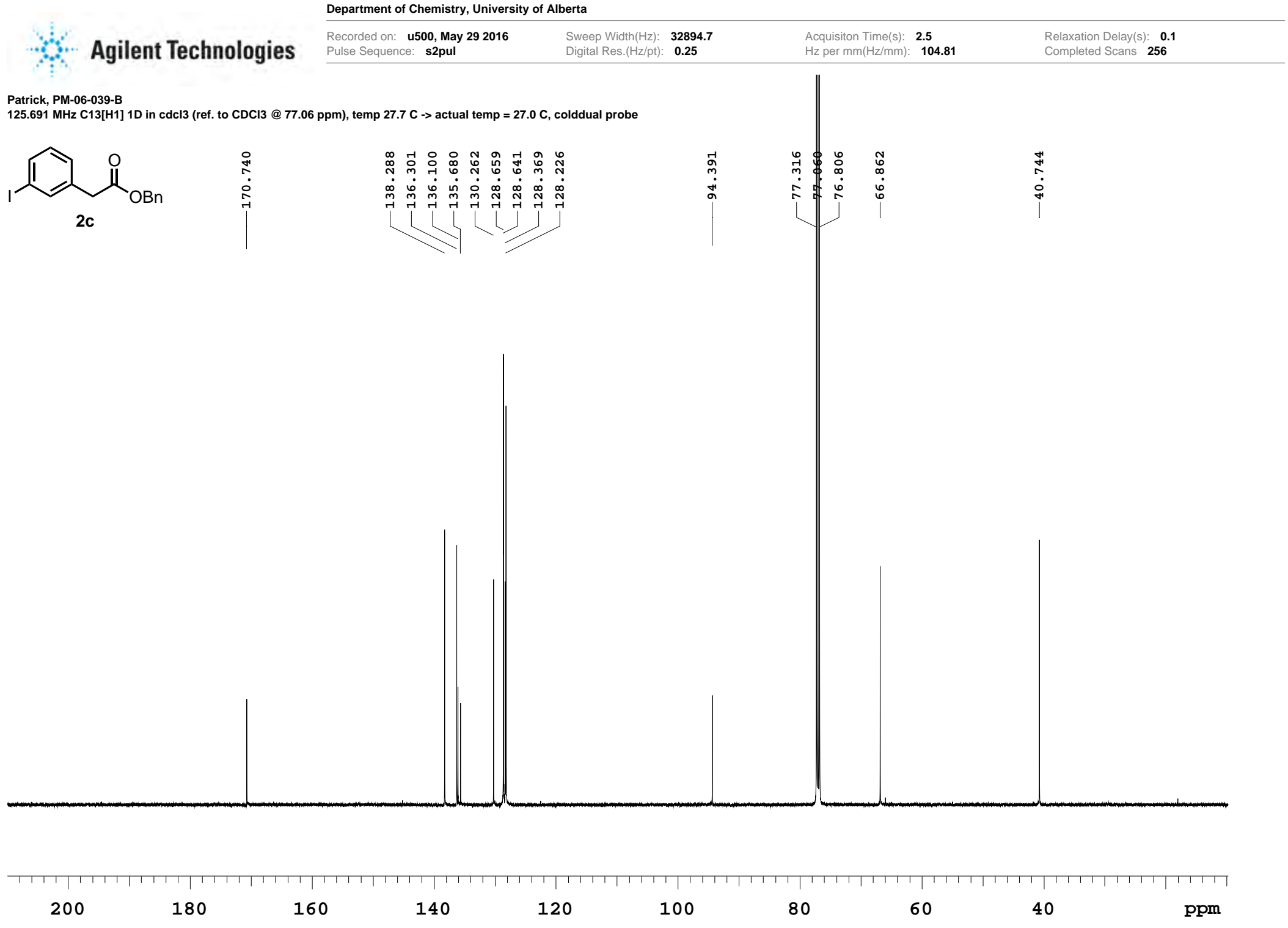

File: /mnt/d600/home13/rijnmr/hmrdata/DATA_FROM_NMRSERVICE/Patrick/2016.05/2016.05.29.u5_PM-06-039-B_loc1_18.59_C13_1D 
Department of Chemistry, University of Alberta

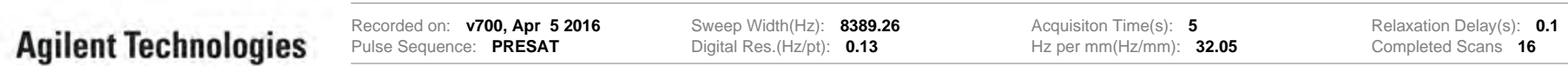

Rylan, RL-05-129-F1

999.762 MHz H1 PRESAT in cdcl3 (ref. to CDCl3 @ 7.26 ppm), temp $27.5 \mathrm{C}$-> actual temp = 27.0 C, coldid probe

$\overbrace{O} \sim_{\mathrm{H}_{4}}$

2d

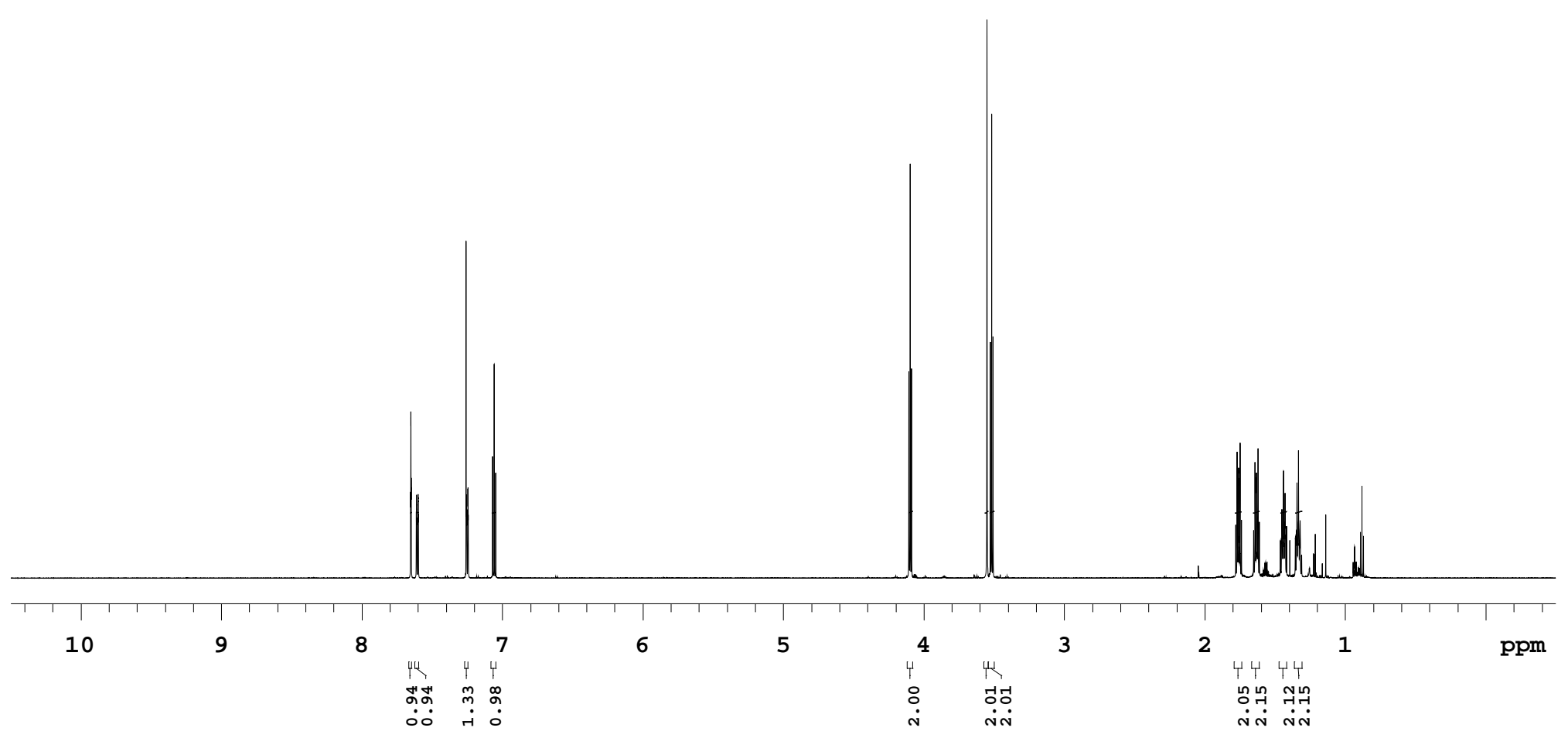

File: /mnt/d600/home13/rilnmr/nmrdata/DATA_FROM_NMRSERVICE/Rylan/2016.04/2016.04.5.v7_RL-05-129-F1_loc1_15.15_H1_1D 
Department of Chemistry, University of Alberta

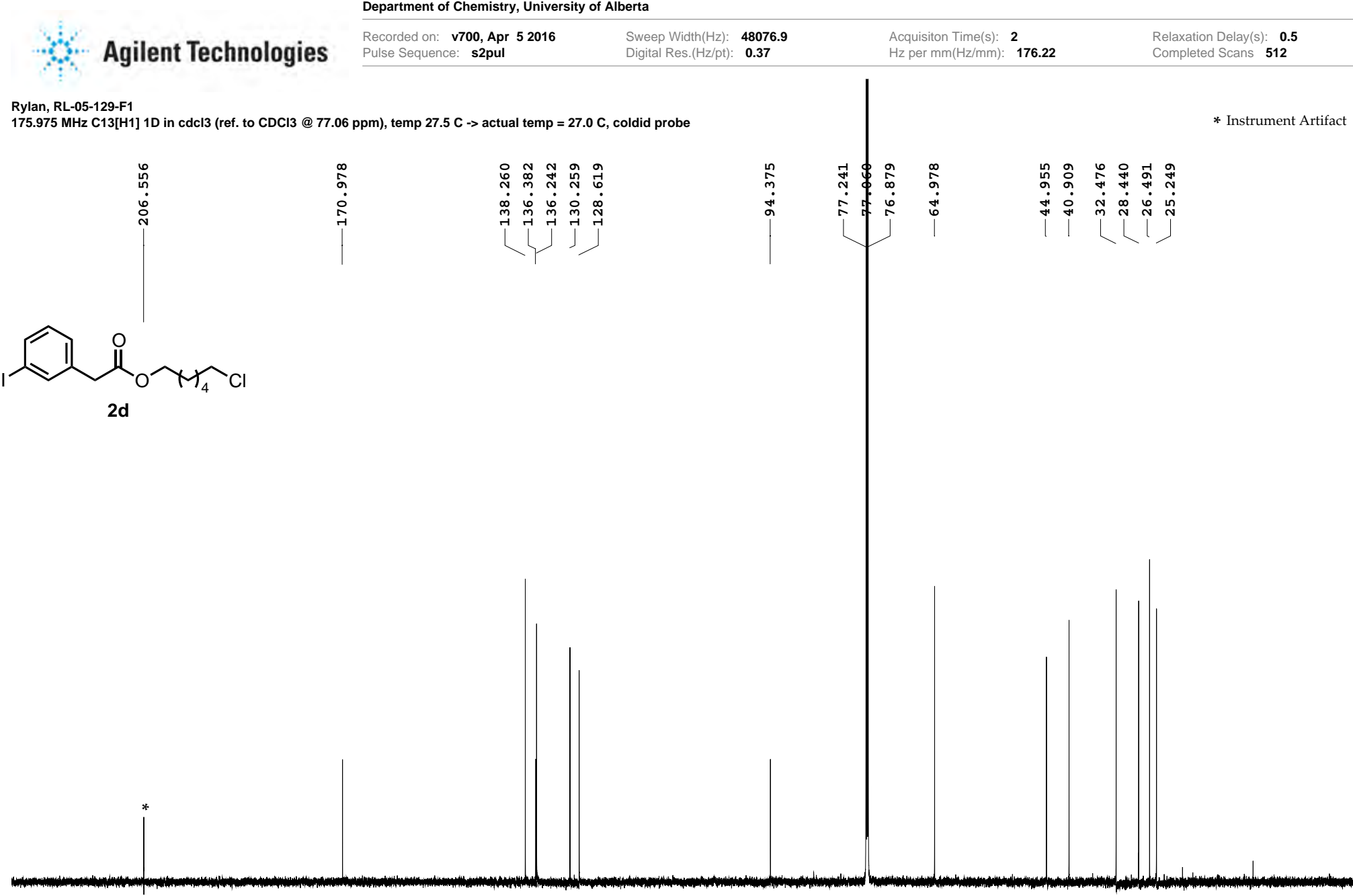
220
200
180
160
140
120
100
80
60
40
20
ppm 
Department of Chemistry, University of Alberta

\begin{tabular}{|c|c|c|c|c|c|c|}
\hline & \\
\hline Agilent Technologies & $\begin{array}{l}\text { Recorded on: } \text { m400, Sep } 222016 \\
\text { Pulse Sequence: PRESAT }\end{array}$ & $\begin{array}{l}\text { Sweep Width(Hz): } 48 \mathrm{C} \\
\text { Digital Res.(Hz/pt): } 0.6\end{array}$ & $\begin{array}{l}803.07 \\
0.07\end{array}$ & $\begin{array}{l}\text { Acquisiton Time(s): } 4.9 \\
\text { Hz per } m m(H z / m m): \\
1 \varepsilon\end{array}$ & $\begin{array}{l}4.997 \\
18.35\end{array}$ & $\begin{array}{l}\text { Relaxation Delay(s): } 0.1 \\
\text { Completed Scans } 64\end{array}$ \\
\hline
\end{tabular}

Shengkang, SY-05-31-DRY

400.389 MHz H1 PRESAT in cdcl3 (ref. to CDCl3 @ $7.26 \mathrm{ppm}$ ), temp $27.0 \mathrm{C}$-> actual temp = 27.0 C, m400gz probe

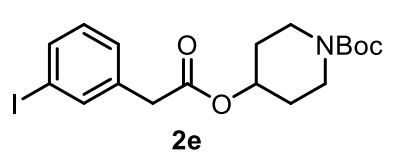

$2 \mathrm{e}$

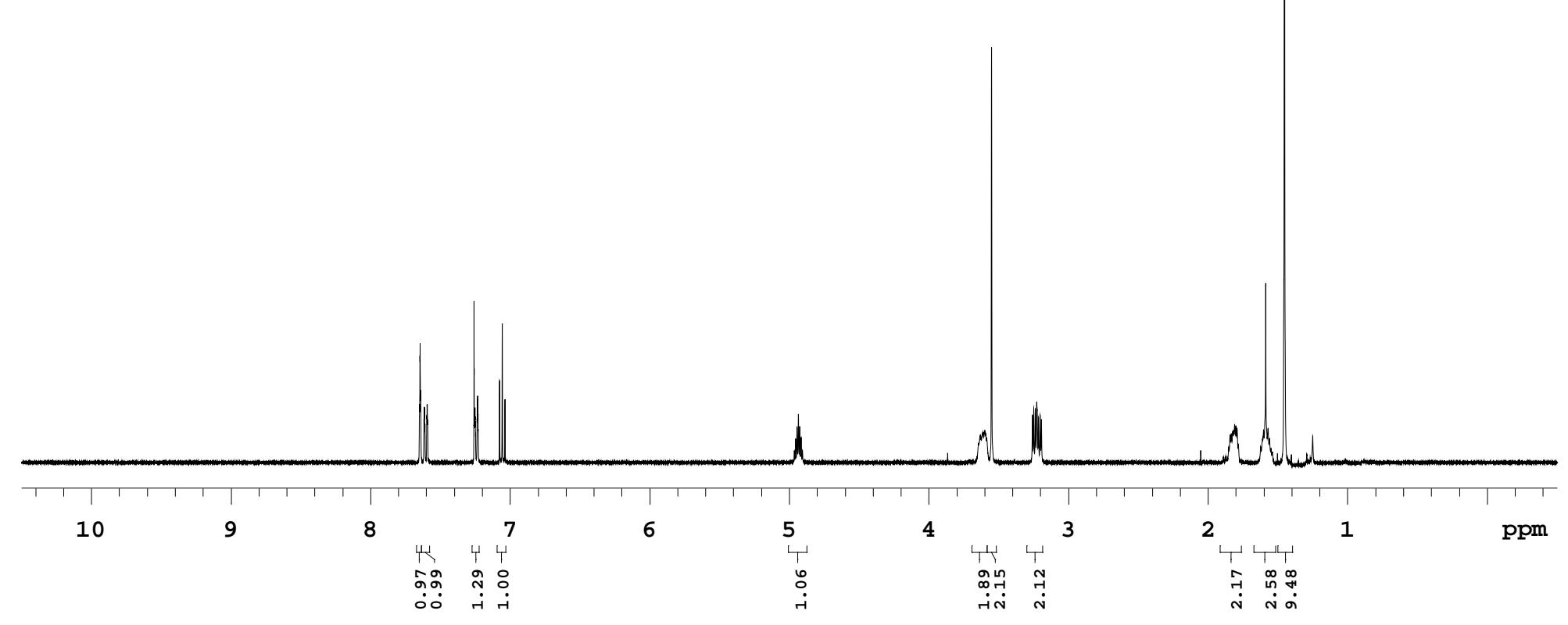

File: /mnt/d600/home12/gennmr/nmrdata/LUNDGREN/Shengkang/2016.09/2016.09.22.m4_SY-05-31-DRY_loc10_14.45_H1_1D 
Department of Chemistry, University of Alberta
Agilent Technologies
Recopided oin v700, Apr 21201 Sweep Widhi $H$ x: 48076 Acquarion Time(s)i, ${ }_{161.29}$ Fiblaxeling Dolizyisy 0.5
48076.9
0.37

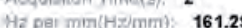 Completes Bcans 1000

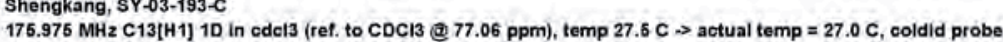
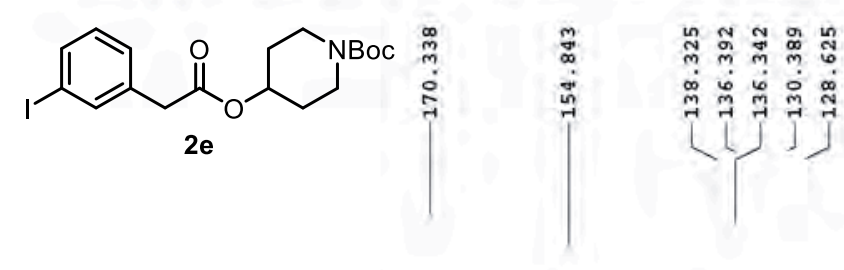

$\stackrel{\circ}{\circ} \dot{\sim}$

[J]
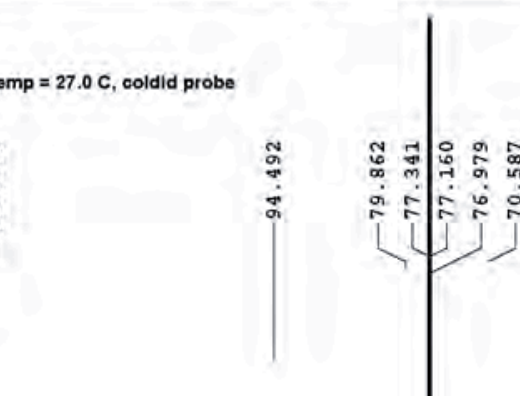

* Instrument Artifact

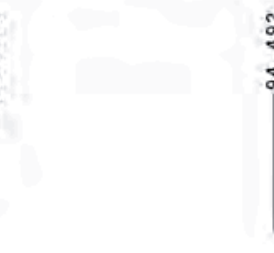

* Instrument Artifact

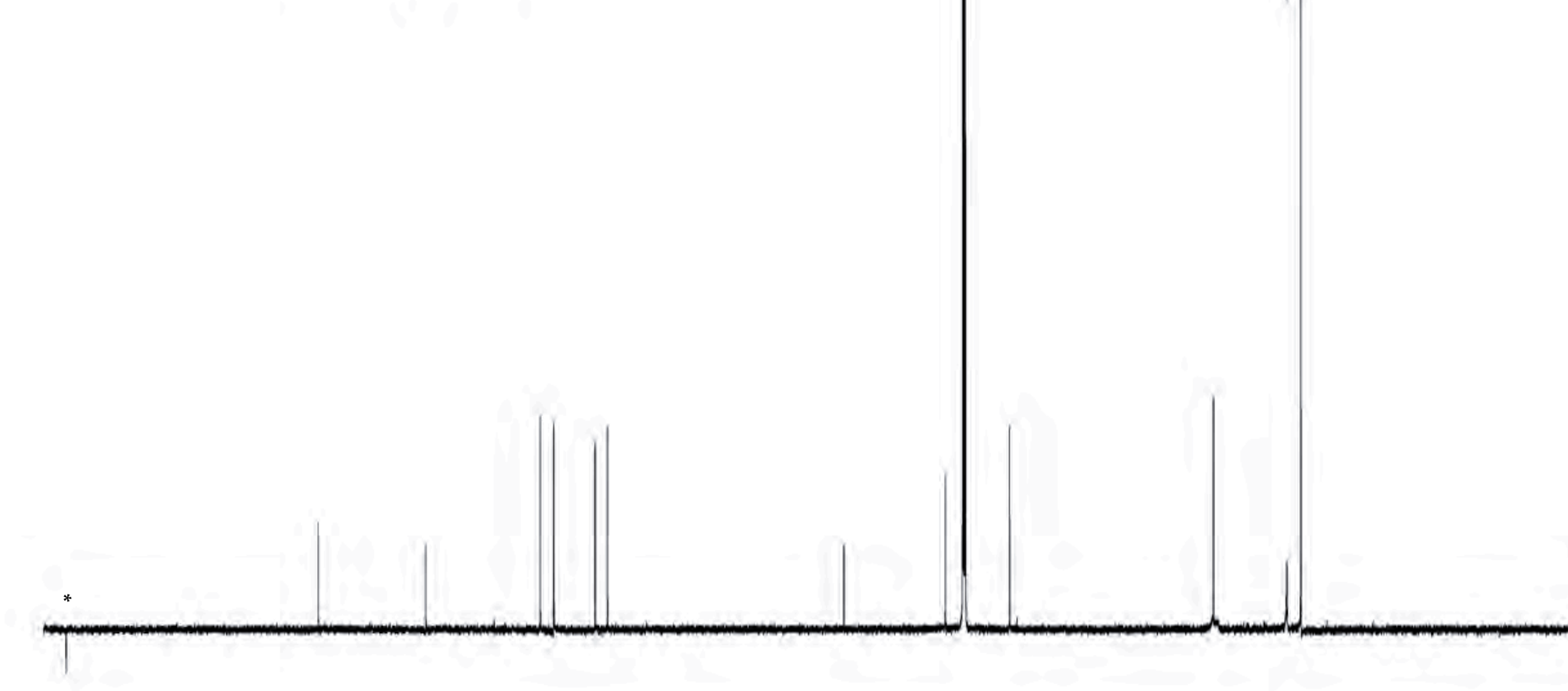

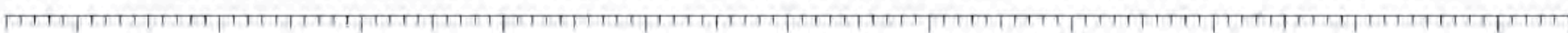


Department of Chemistry, University of Alberta

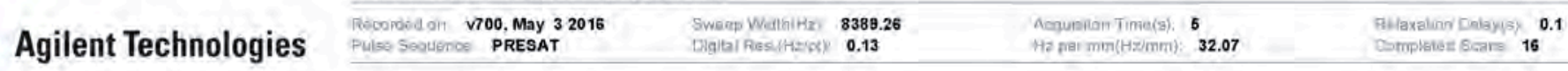

Shengkang, SY-03-199-1-A

$699.762 \mathrm{MHz}$ H1 PRESAT in cdel3 (ret. to CDC13 @ $7.26 \mathrm{ppm}$ ), temp $27.5 \mathrm{C} \rightarrow$ actual temp $=27.0 \mathrm{C}$, coldid probe

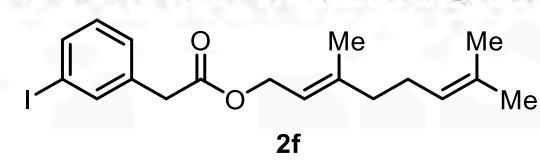

$2 f$

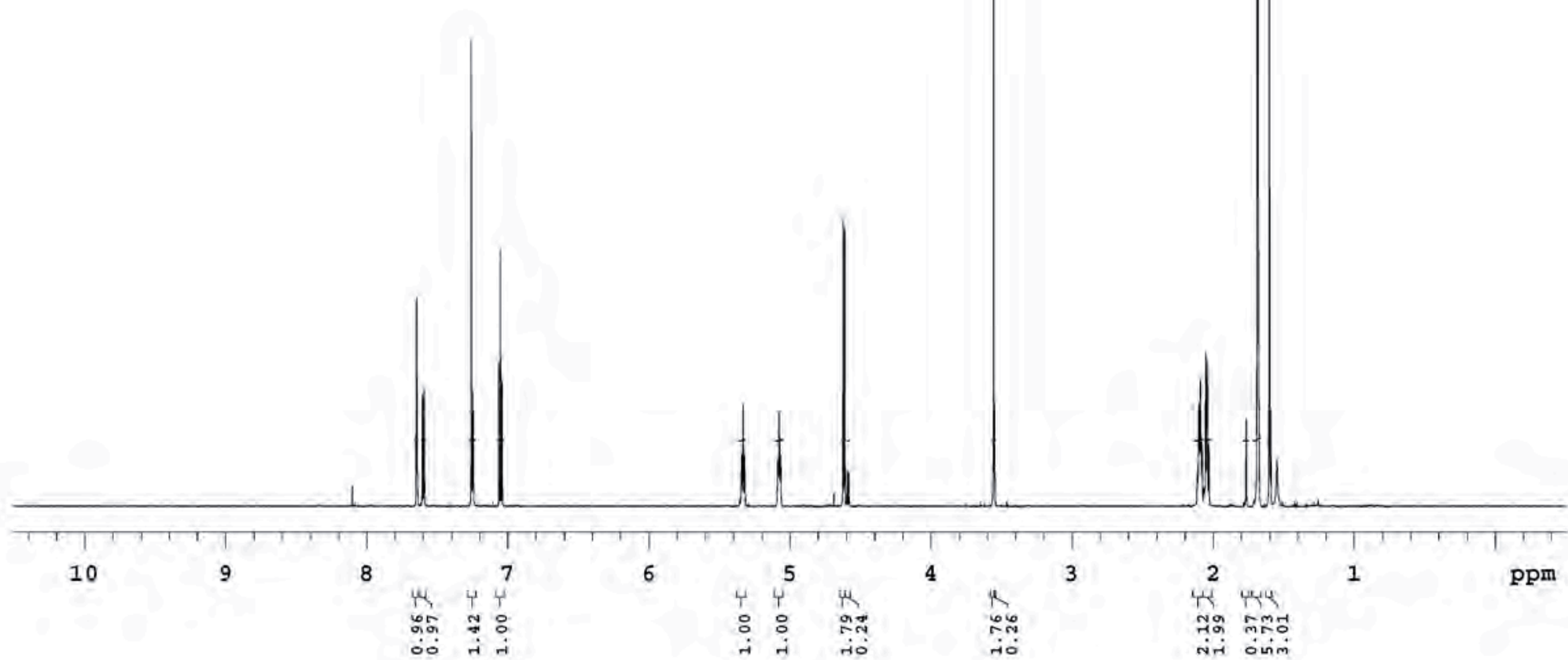




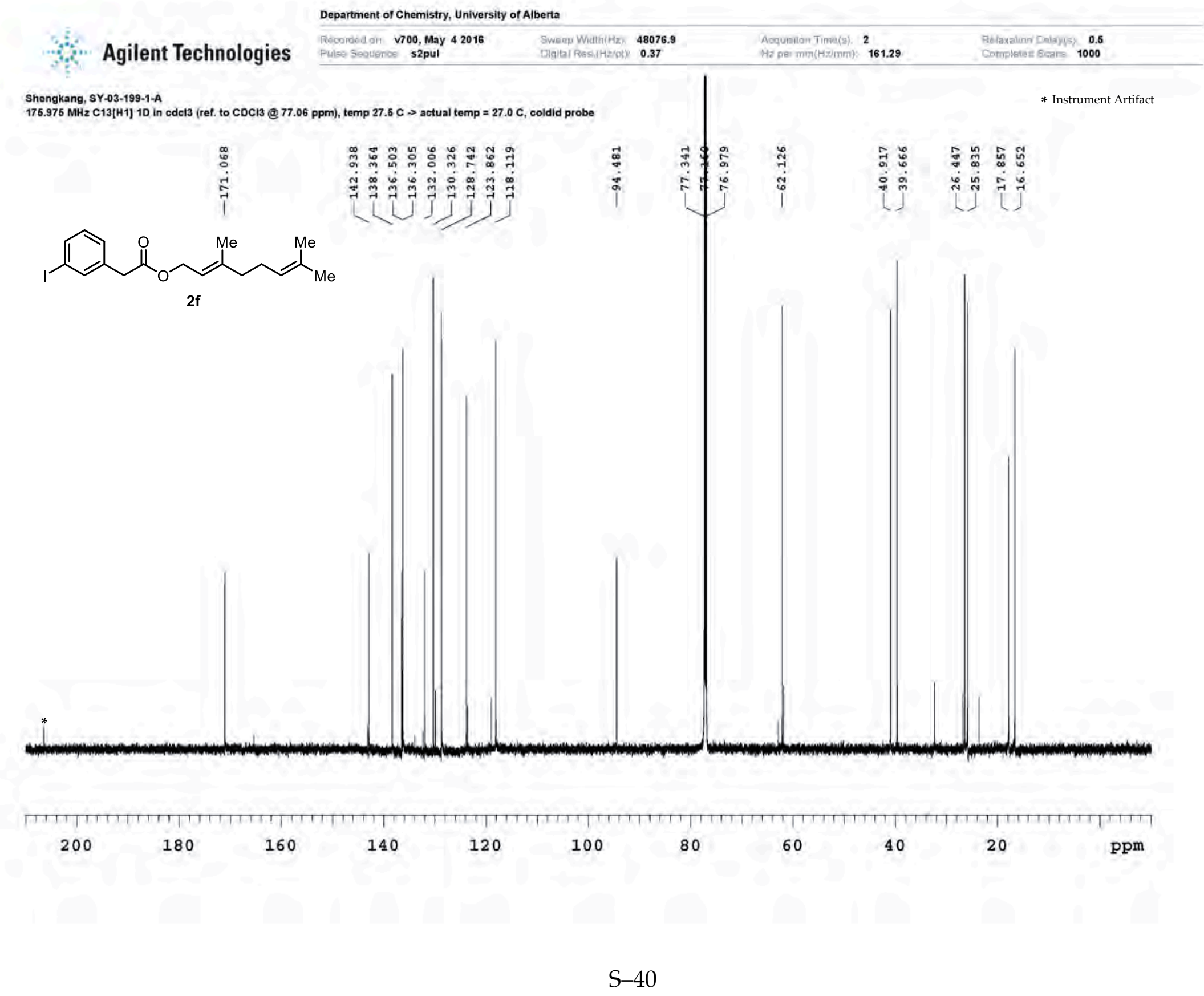


Department of Chemistry, University of Alberta

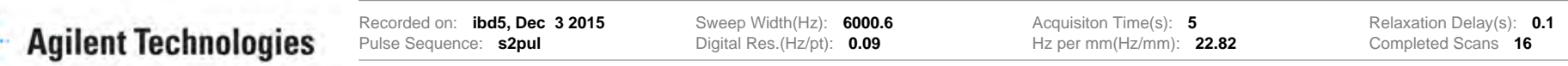

RL-05-61 $498.118 \mathrm{MHz}$ H1 $1 \mathrm{D}$ in cdcl3 (ref. to CDCl3 @ $7.26 \mathrm{ppm}$ ), temp $26.4 \mathrm{C}$-> actual temp = 27.0 C, autoxdb probe<smiles>CCOC(=O)Cc1cccc(Br)c1</smiles>

2g

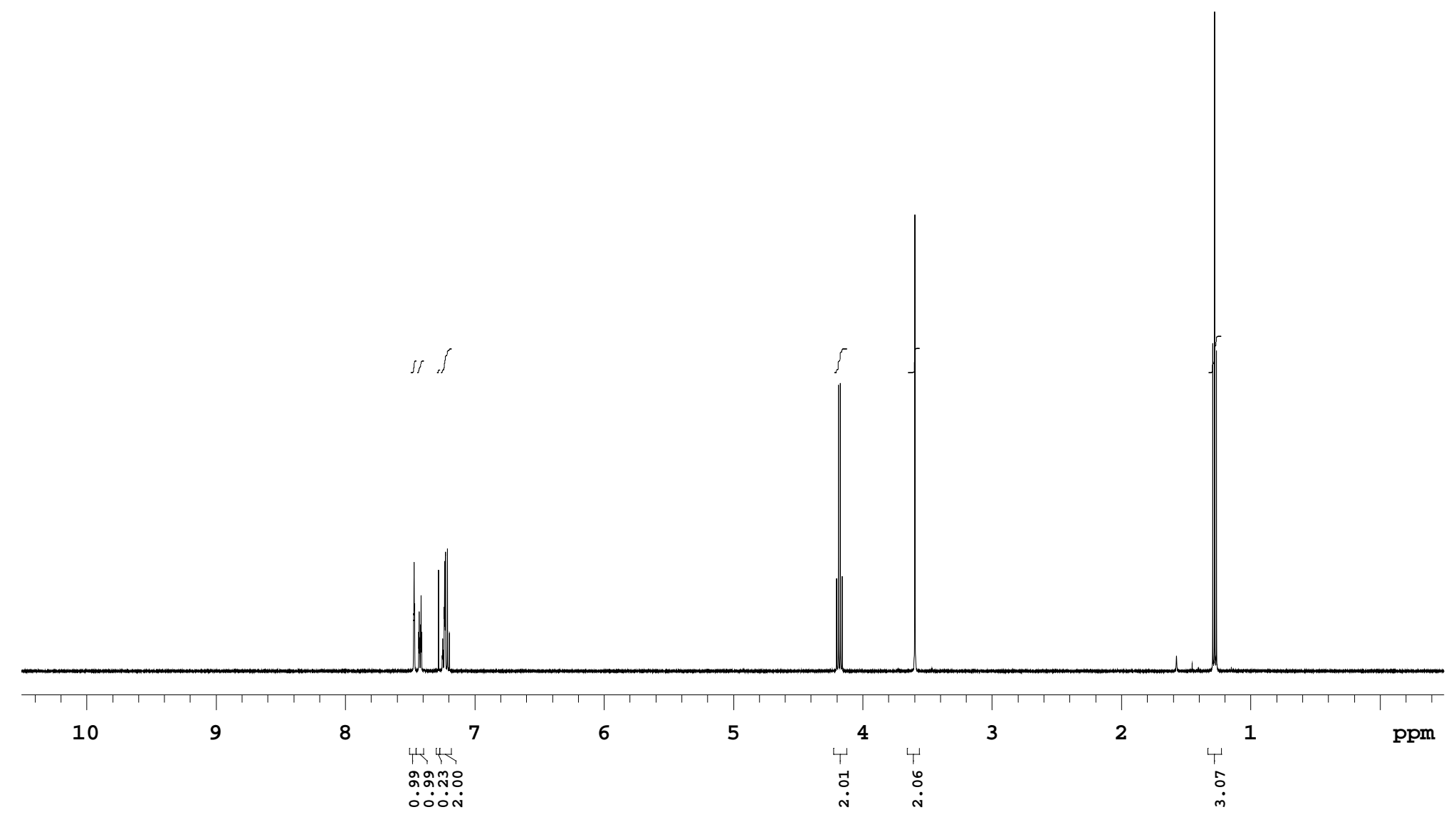

File: /mnt/d600/home13/ri.hnmr/nmrdata/Rylan/2015.12/2015.12.03.15_RL-05-61_H1_1D 
Department of Chemistry, University of Alberta

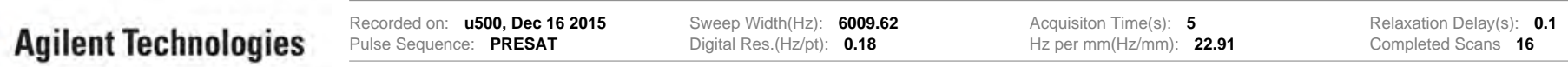

Rylan, RL-05-83-CC2

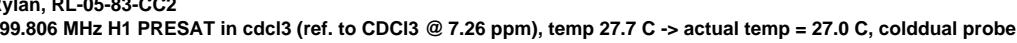

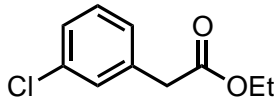

2h

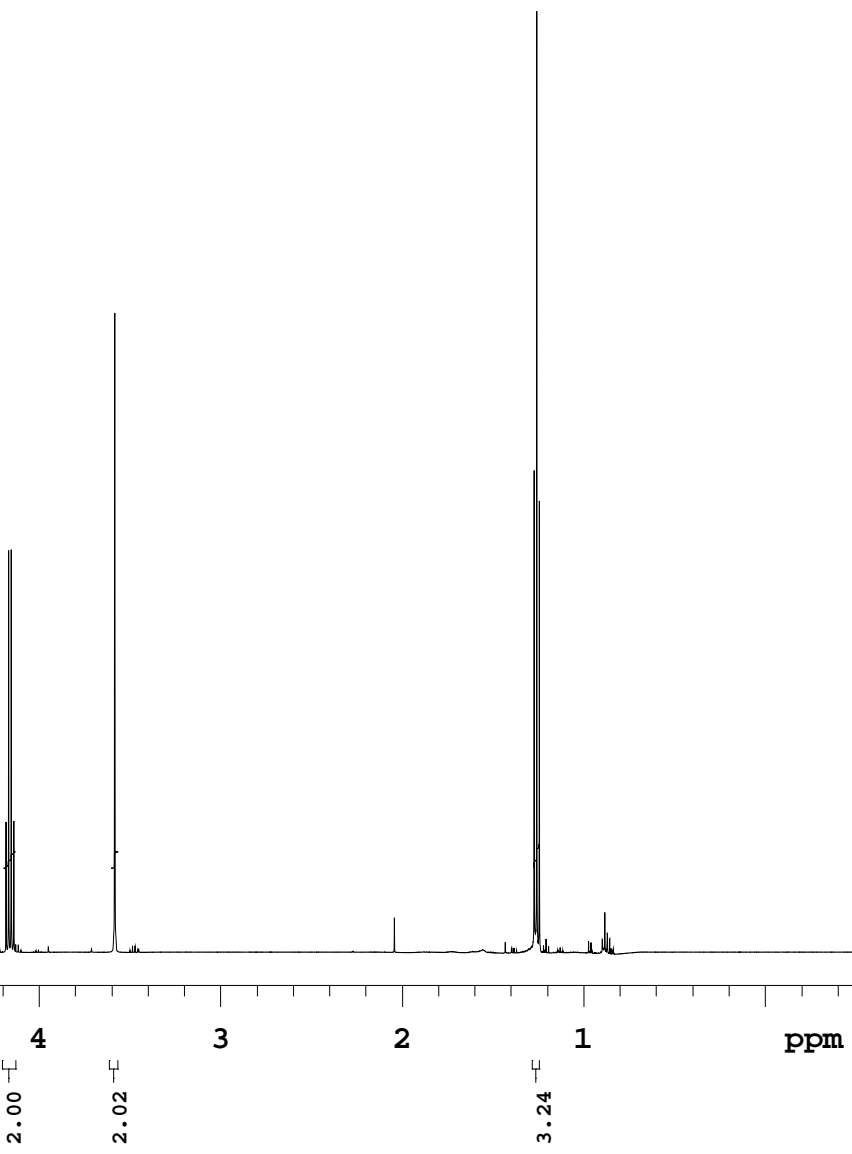

File: /mnt/d600/home13/rilnmr/nmrdata/DATA_FROM_NMRSERVICE/Rylan/2015.12/2015.12.16.u5_RL-05-83-CC2_loc9_18.14_H1_1D 
Department of Chemistry, University of Alberta

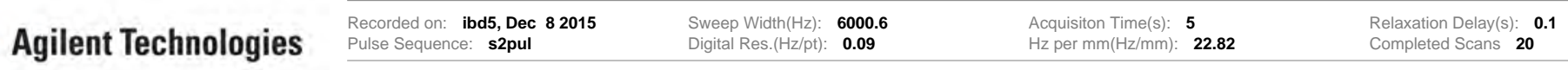

PM-06-067-C-F14-15-2

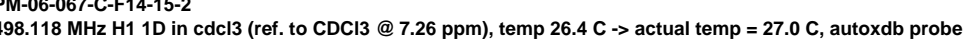

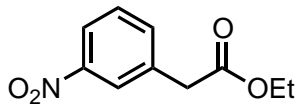

2i

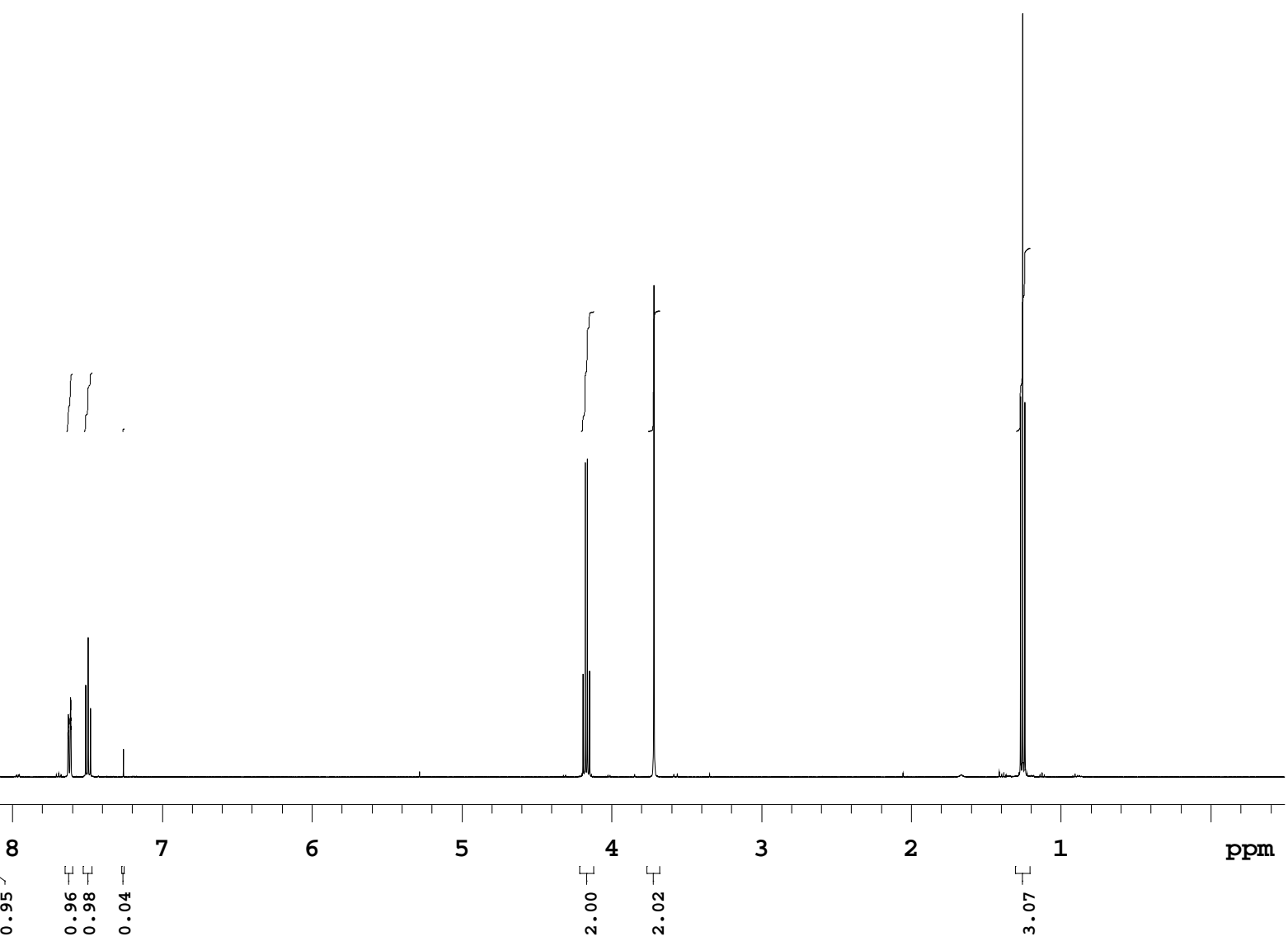

File: /mnt/d600/home13/jilnmr/nmrdata/Patrick/2015.12/2015.12.08.15_PM-06-067-C-F14-15-2_H1_1D 
Department of Chemistry, University of Alberta

\begin{tabular}{|c|c|c|c|}
\hline Arilent Technolnaios & $\begin{array}{l}\text { Recorded on: ibd5, Dec } 112015 \\
\text { Pulse Sequence: s2pul }\end{array}$ & $\begin{array}{l}\text { Acquisiton Time(s): } \mathbf{5} \\
\text { Hz per } \mathrm{mm}(\mathrm{Hz} / \mathrm{mm}): \mathbf{2 2 . 8 5}\end{array}$ & $\begin{array}{l}\text { Relaxation Delay(s): } 0.1 \\
\text { Completed Scans } \mathbf{1 6}\end{array}$ \\
\hline
\end{tabular}

RL-05-69

(208.118 MHz H1 1D in cdcl3 (ref. to CDCl3 @ $7.26 \mathrm{ppm}$ ), temp $26.4 \mathrm{C}$-> actual temp = 27.0 C, autoxdb probe

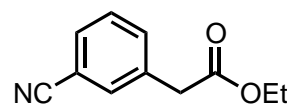

2j

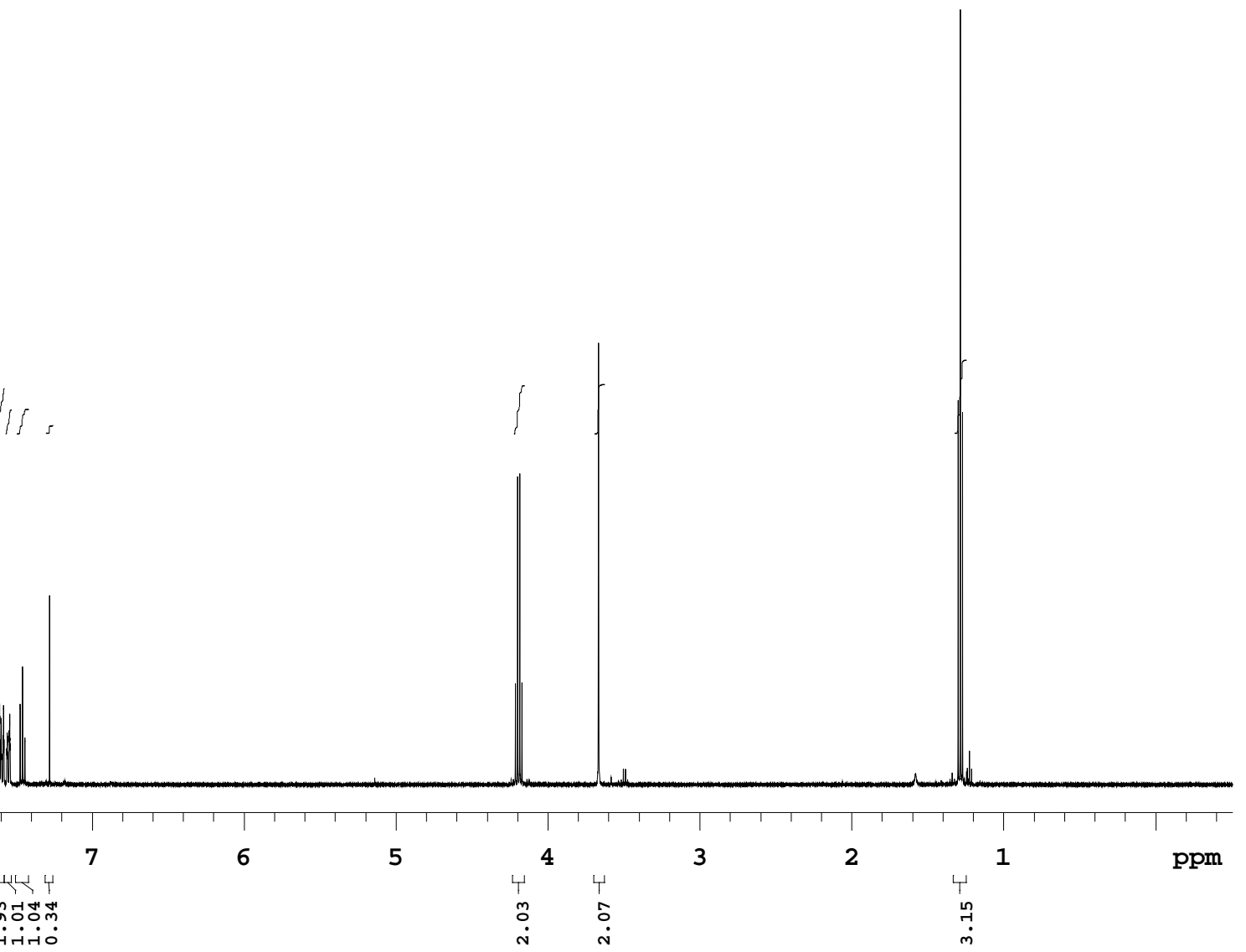

File: /mnt/d600/home13/rijnmr/hmrdata/Rylan/2015.12/2015.12.11.15_RL-05-69_H1_1D 
Department of Chemistry, University of Alberta

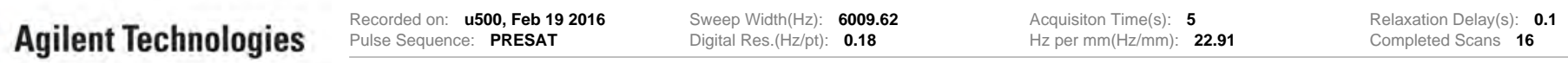

Patrick, PM-07-017-F19-22

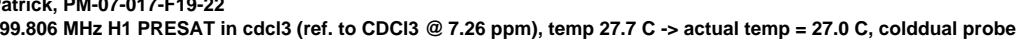

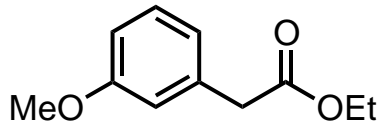

$\mathbf{2 k}$

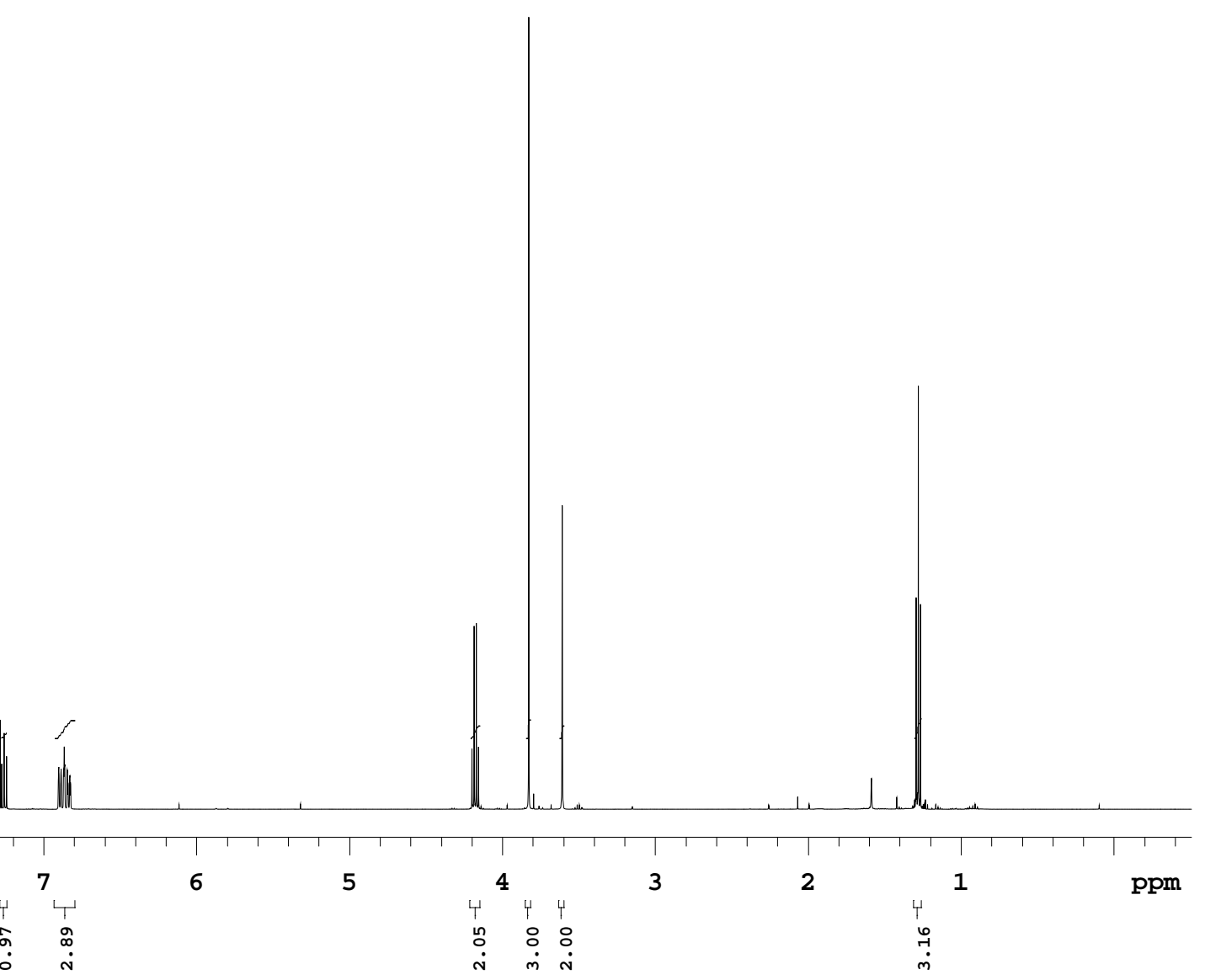

File: /mnt/d600/home13/rilnmr/nmrdata/DATA_FROM_NMRSERVICE/Patrick/2016.02/2016.02.19.u5_PM-07-017-F19-22_loc2_07.09_H1_1D 
Department of Chemistry, University of Alberta

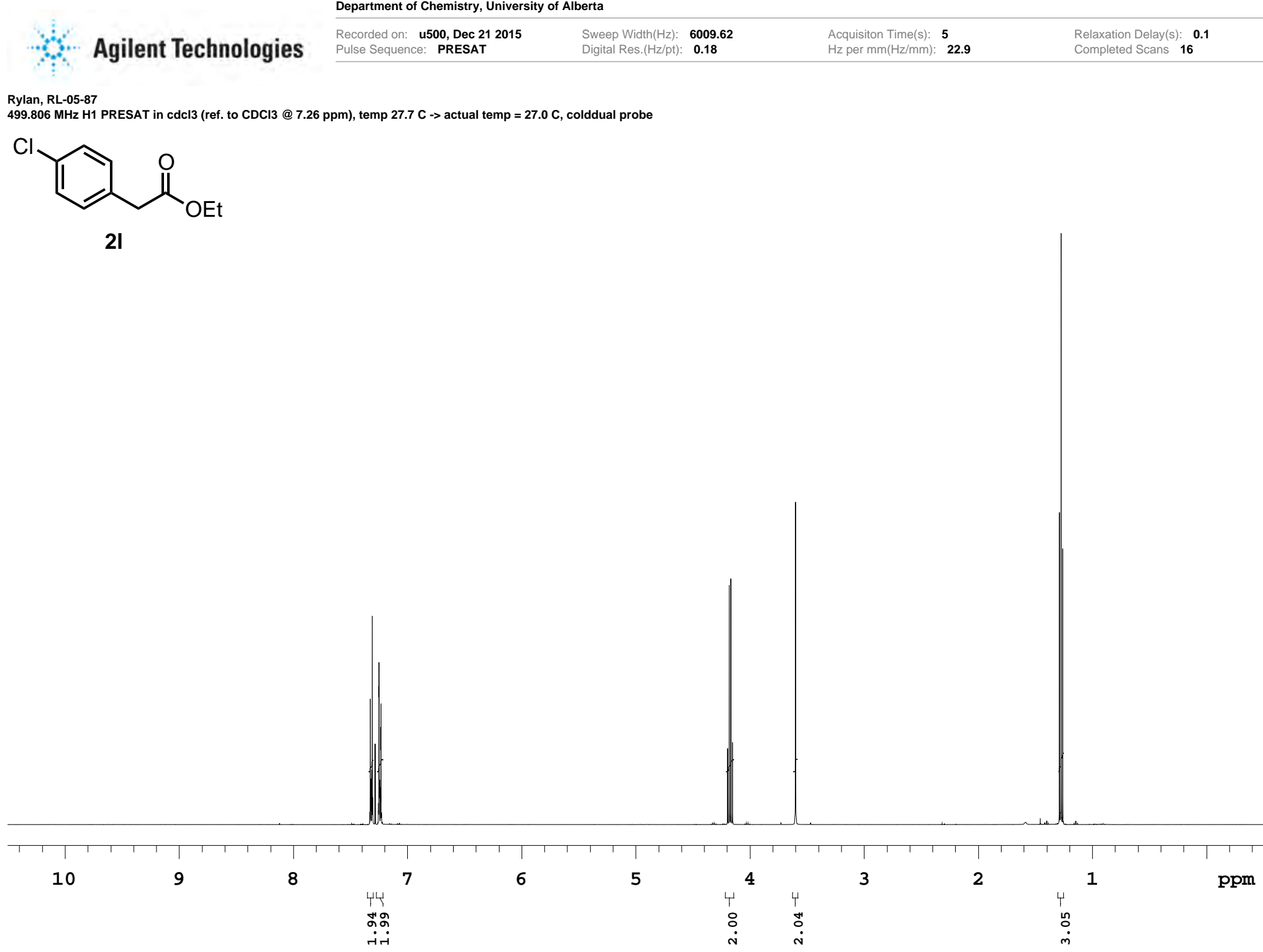

File: /mnt/d600/home13/rijnmr/hmrdata/DATA_FROM_NMRSERVICE/Rylan/2015.12/2015.12.21.u5_RL-05-87_loc7_03.55_H1_1D 
Department of Chemistry, University of Alberta

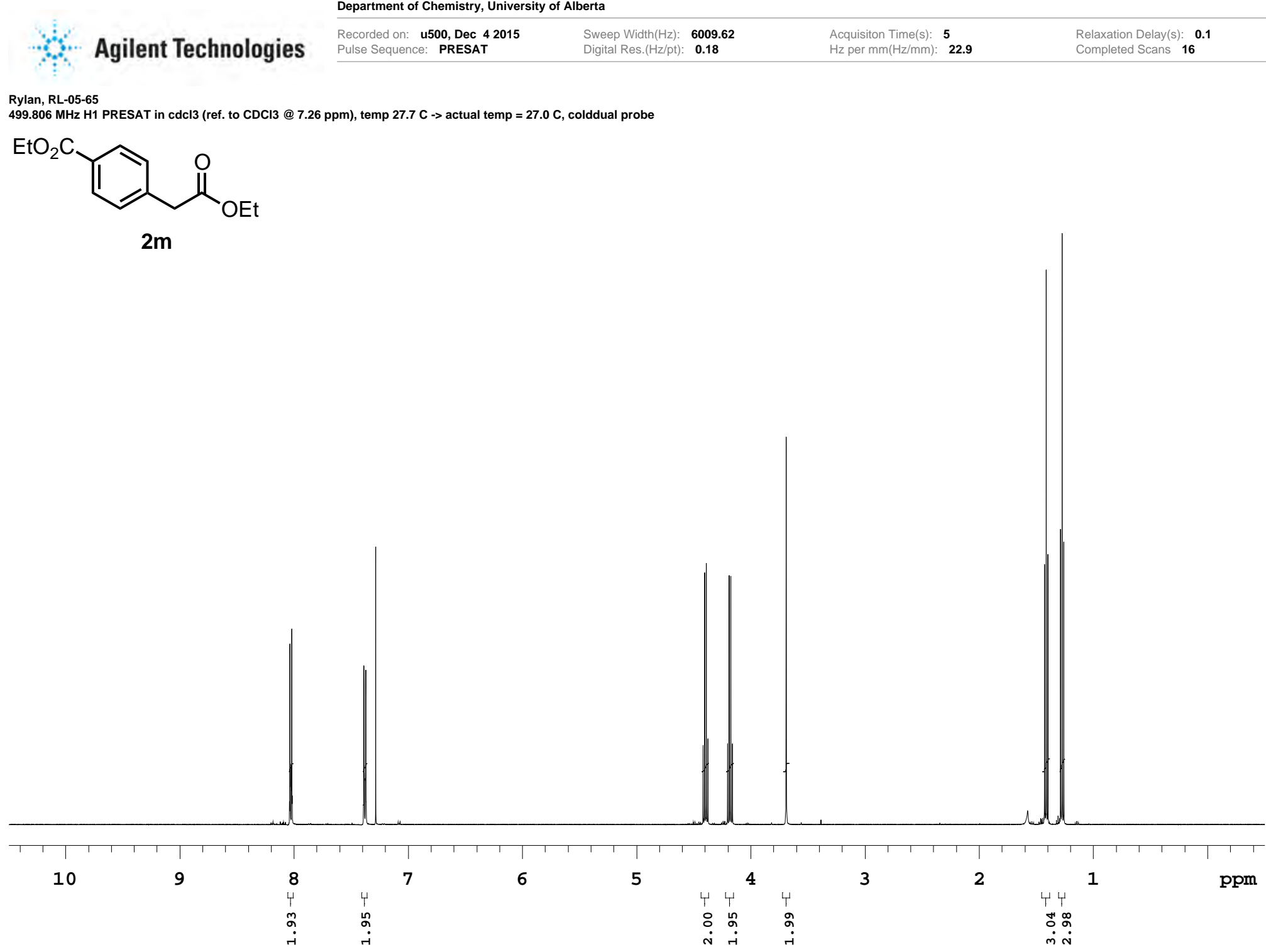

File: /mnt/d600/home13/rijnmr/nmrdata/DATA_FROM_NMRSERVICE/Rylan/2015.12/2015.12.04.u5_RL-05-65_loc7_04.24_H1_1D 
Department of Chemistry, University of Alberta

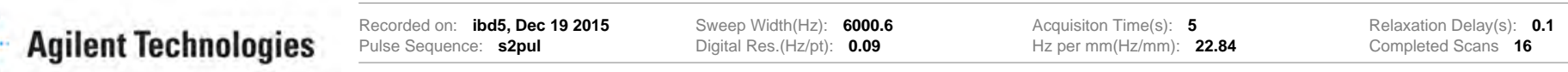

RL-05-91

498.118 MHz H1 1D in cdcl3 (ref. to CDCl3 @ 7.26 ppm), temp $26.4 \mathrm{C}->$ actual temp = 27.0 C, autoxdb probe

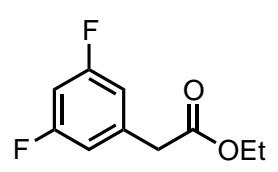

$2 n$

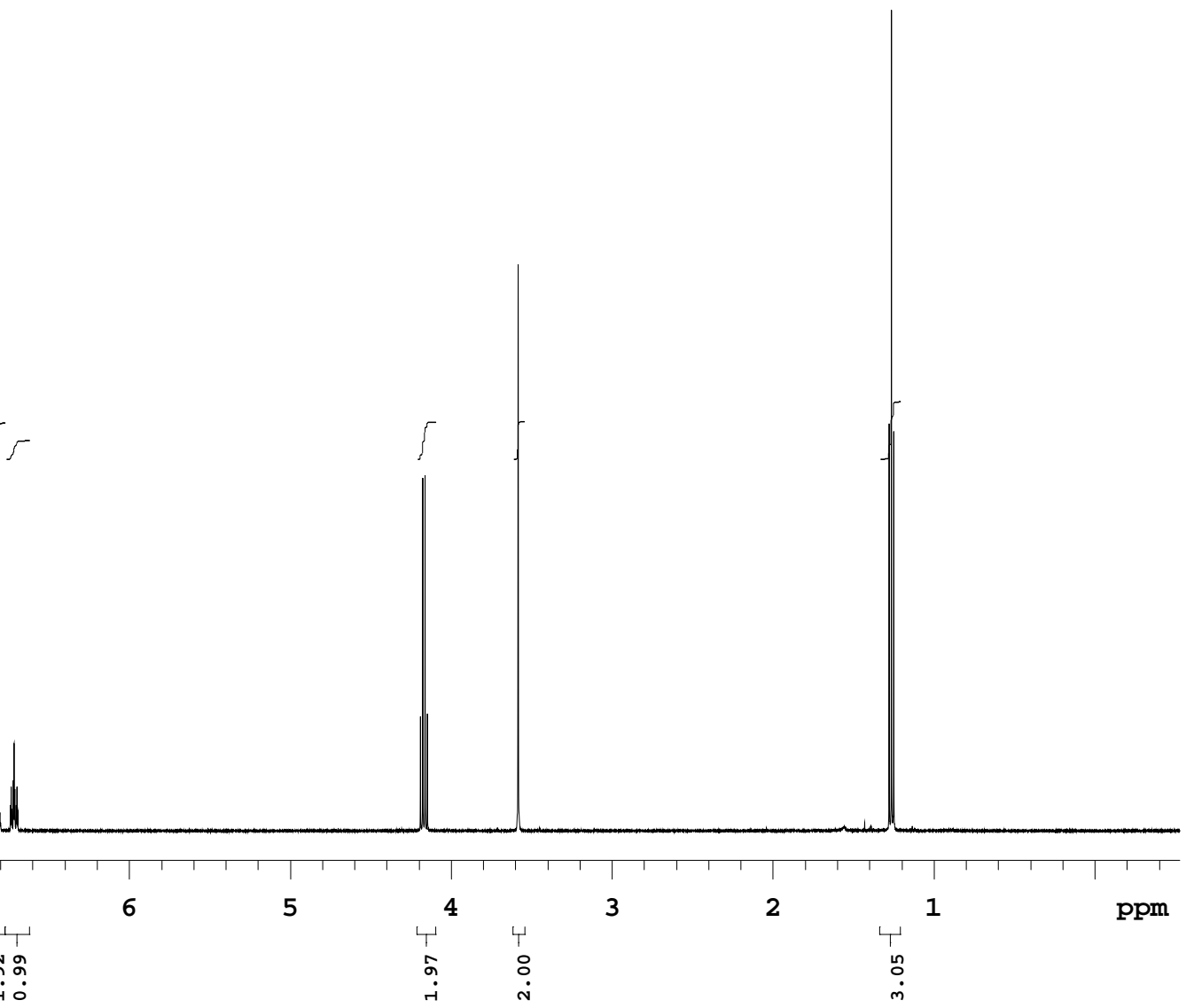

File: /mnt/d600/home13/rilnmr/nmrdata/Rylan/2015.12/2015.12.19.15_RL-05-91_H1_1D 


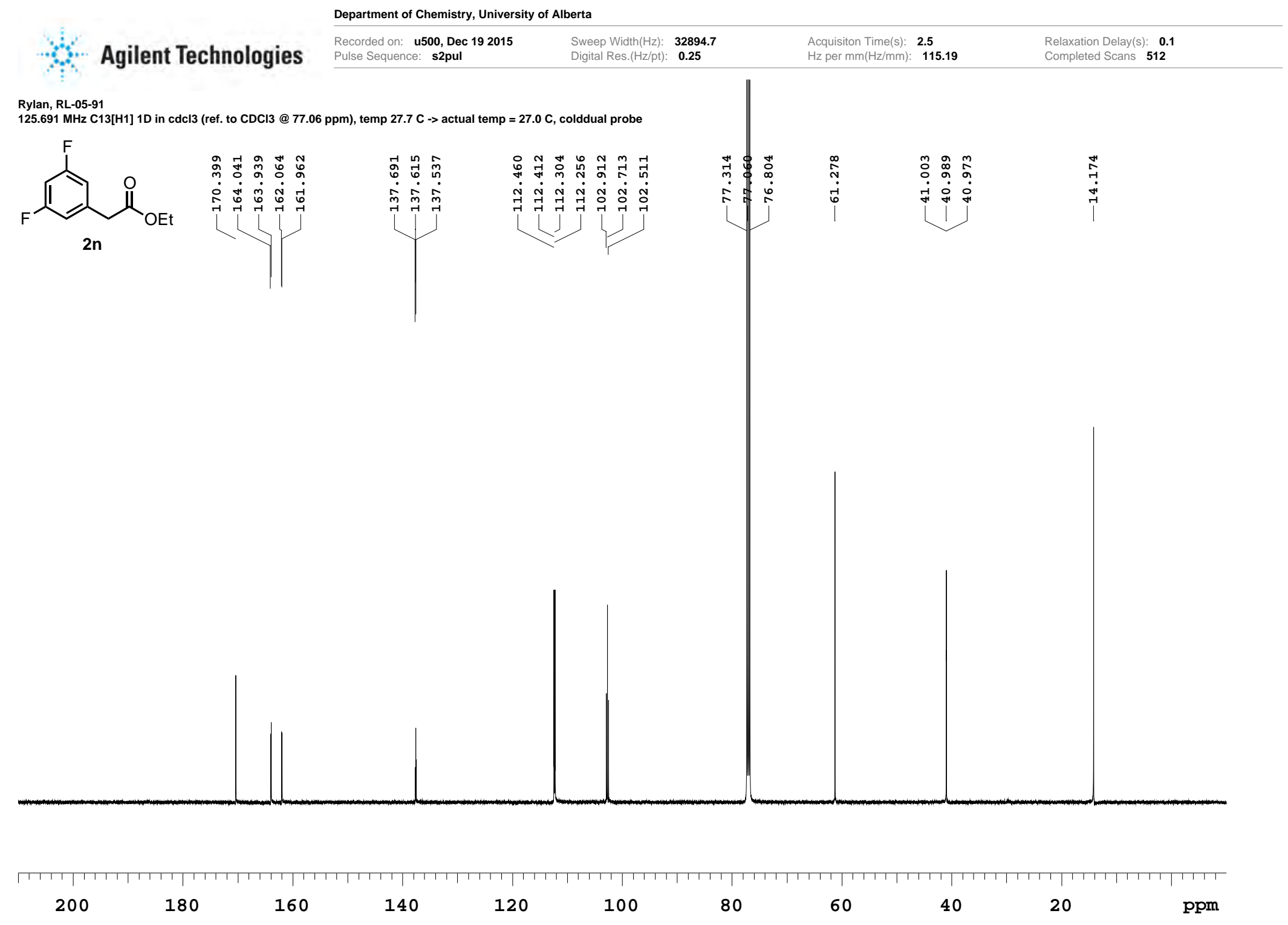

File: /mnt/d600/home13/rilnmr/nmrdata/DATA_FROM_NMRSERVICE/Rylan/2015.12/2015.12.19.u5_RL-05-91_loc5_20.09_C13_1D 
Department of Chemistry, University of Alberta

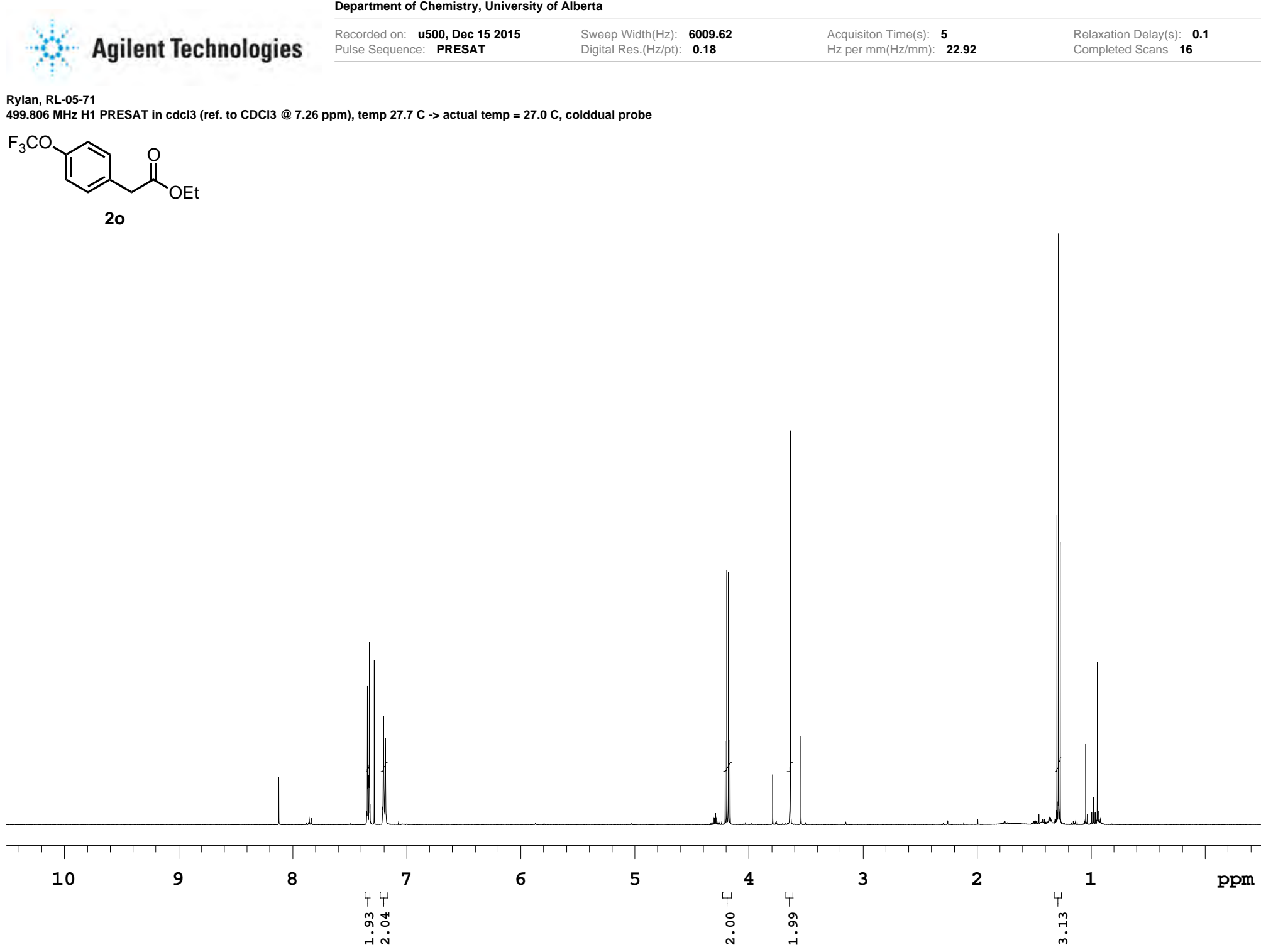

File: /mnt/d600/home13/rijnmr/nmrdata/DATA_FROM_NMRSERVICE/Rylan/2015.12/2015.12.15.u5_RL-05-71_loc6_19.35_H1_1D 
Department of Chemistry, University of Alberta

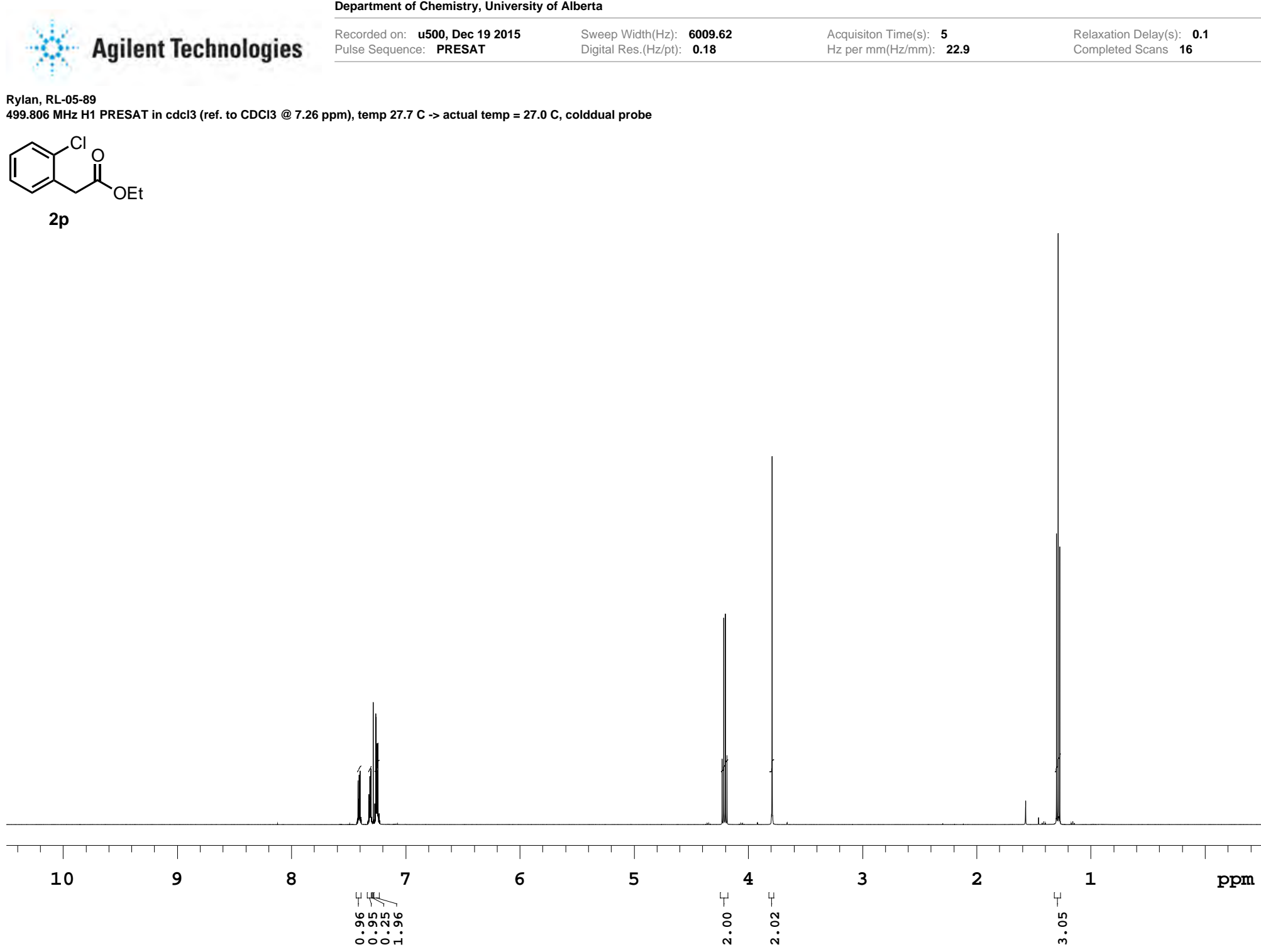

File: /mnt/d600/home13/rijnmr/hmrdata/DATA_FROM_NMRSERVICE/Rylan/2015.12/2015.12.19.u5_RL-05-89_loc6_20.36_H1_1D 
Department of Chemistry, University of Alberta

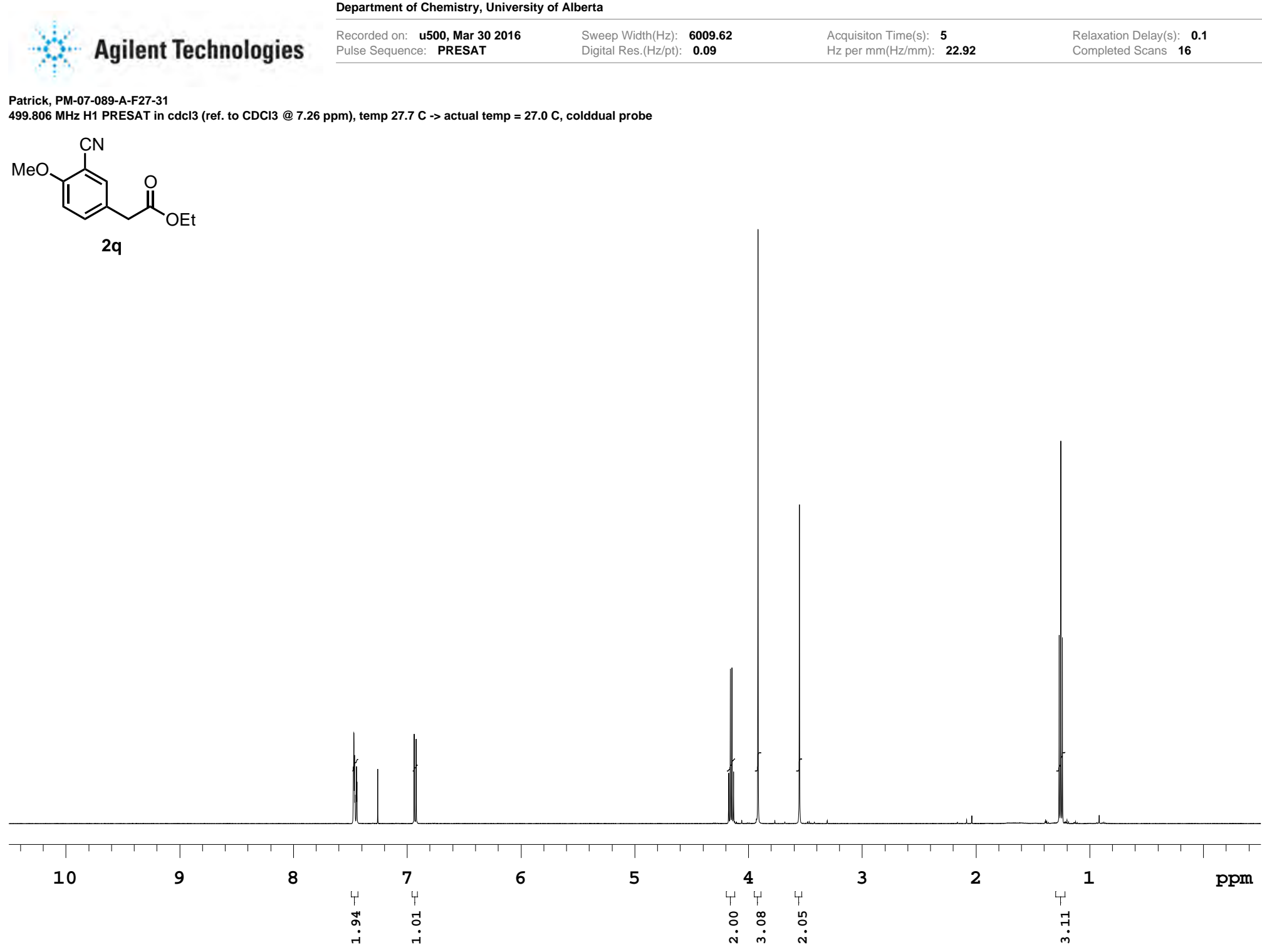

File: /mnt/d600/home13/rijnmr/hmrdata/DATA_FROM_NMRSERVICE/Patrick/2016.03/2016.03.30.u5_PM-07-089-A-F27-31_loc9_07.29_H1_1D 
Department of Chemistry, University of Alberta

\begin{tabular}{|c|c|c|c|c|}
\hline Aailent Techn & $\begin{array}{l}\text { Recorded on: u500, Mar } 302016 \\
\text { Pulse Sequence: s2pul }\end{array}$ & $\begin{array}{l}\text { Sweep Width(Hz): } \quad \mathbf{3 2 8 9 4 . 7} \\
\text { Digital Res.(Hz/pt): } \mathbf{0 . 2 5}\end{array}$ & $\begin{array}{ll}\text { Acquisiton Time(s): } & \mathbf{2 . 5} \\
\mathrm{Hz} \text { per } \mathrm{mm}(\mathrm{Hz} / \mathrm{mm}): & \mathbf{1 1 5 . 3 3}\end{array}$ & $\begin{array}{l}\text { Relaxation Delay(s): } \mathbf{0 . 1} \\
\text { Completed Scans } \mathbf{2 5 6}\end{array}$ \\
\hline
\end{tabular}

Patrick, PM-07-089-A-F27-31

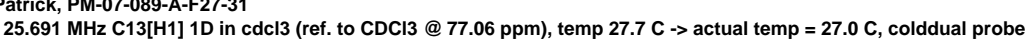

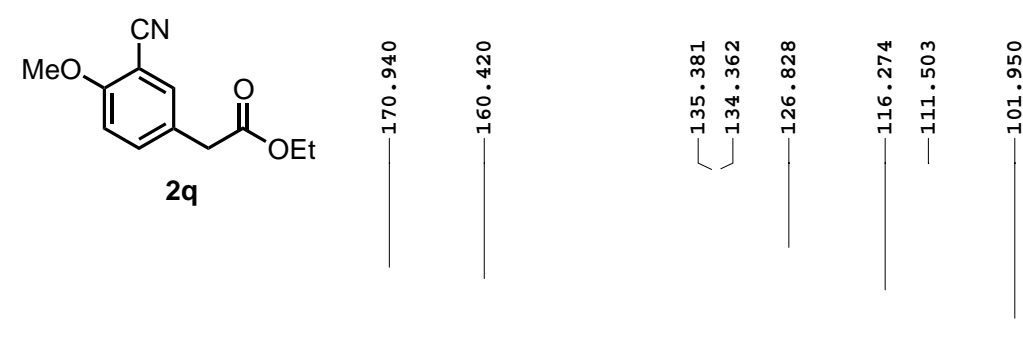

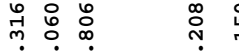

i⿱

กั

$\stackrel{\infty}{\rightarrow+}$

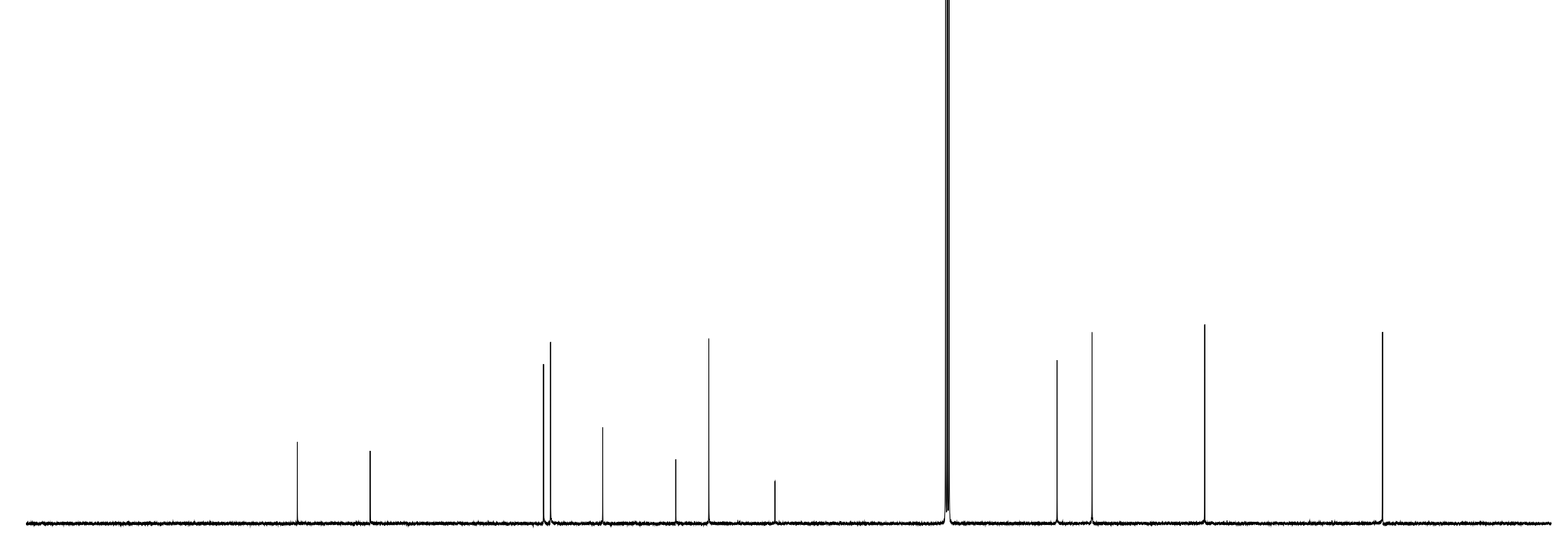

200

180

160

140

120

100

80

60

40

20

ppm 
Department of Chemistry, University of Alberta

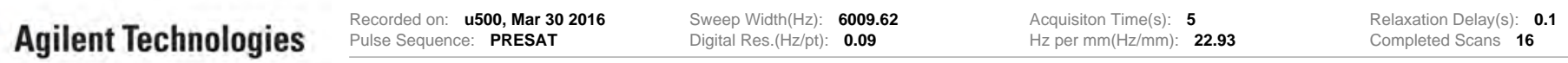

Patrick, PM-07-089-B-F10-19

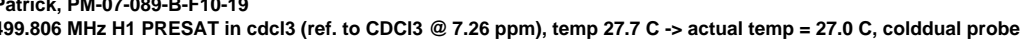

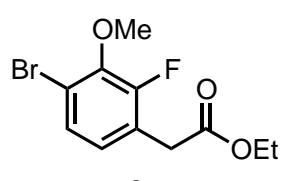

$2 \mathrm{r}$

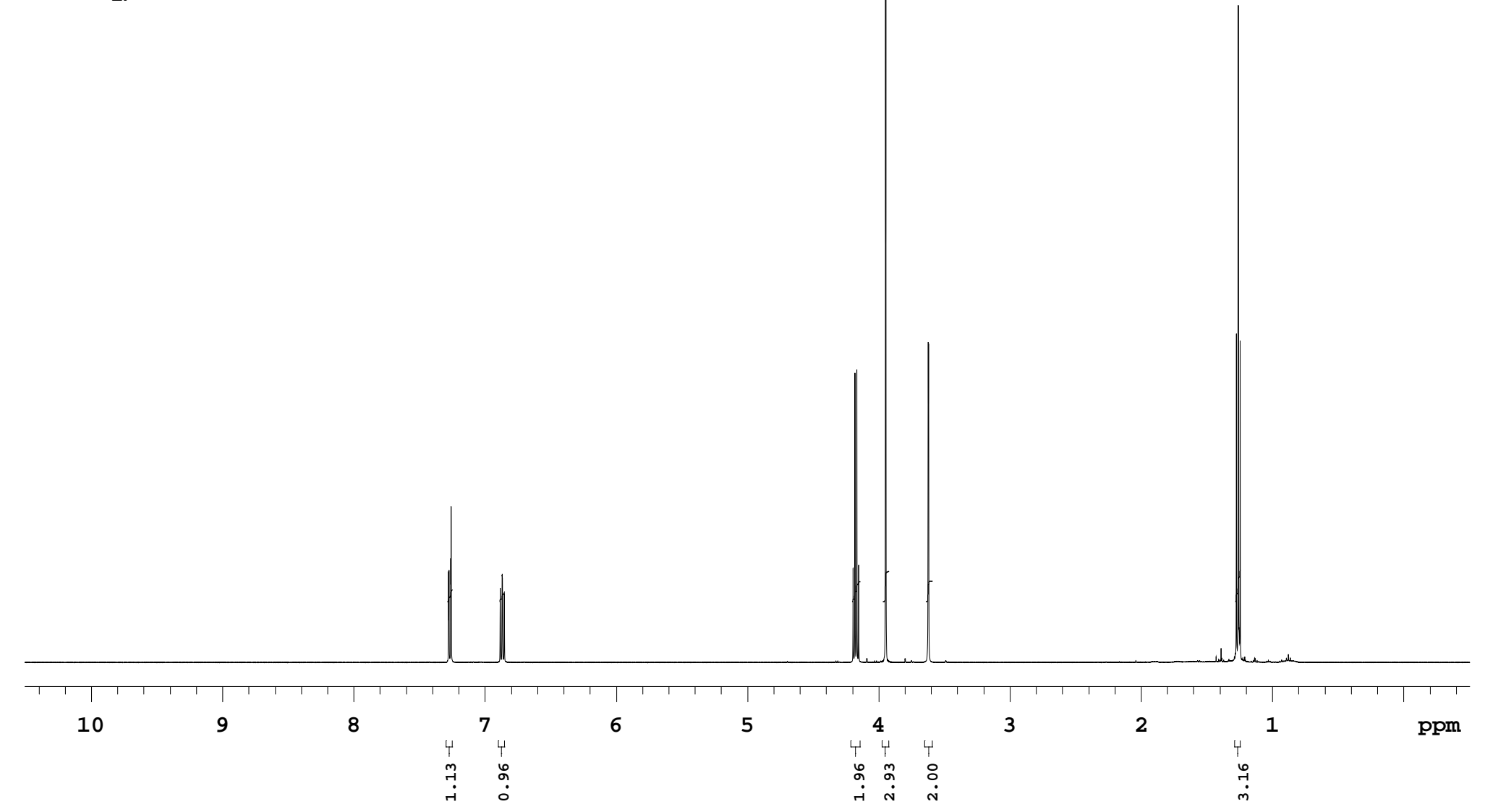

File: /mnt/d600/home13/rijnmr/nmrdata/DATA_FROM_NMRSERVICE/Patrick/2016.03/2016.03.30.u5_PM-07-089-B-F10-19_loc10_07.46_H1_1D 
Department of Chemistry, University of Alberta

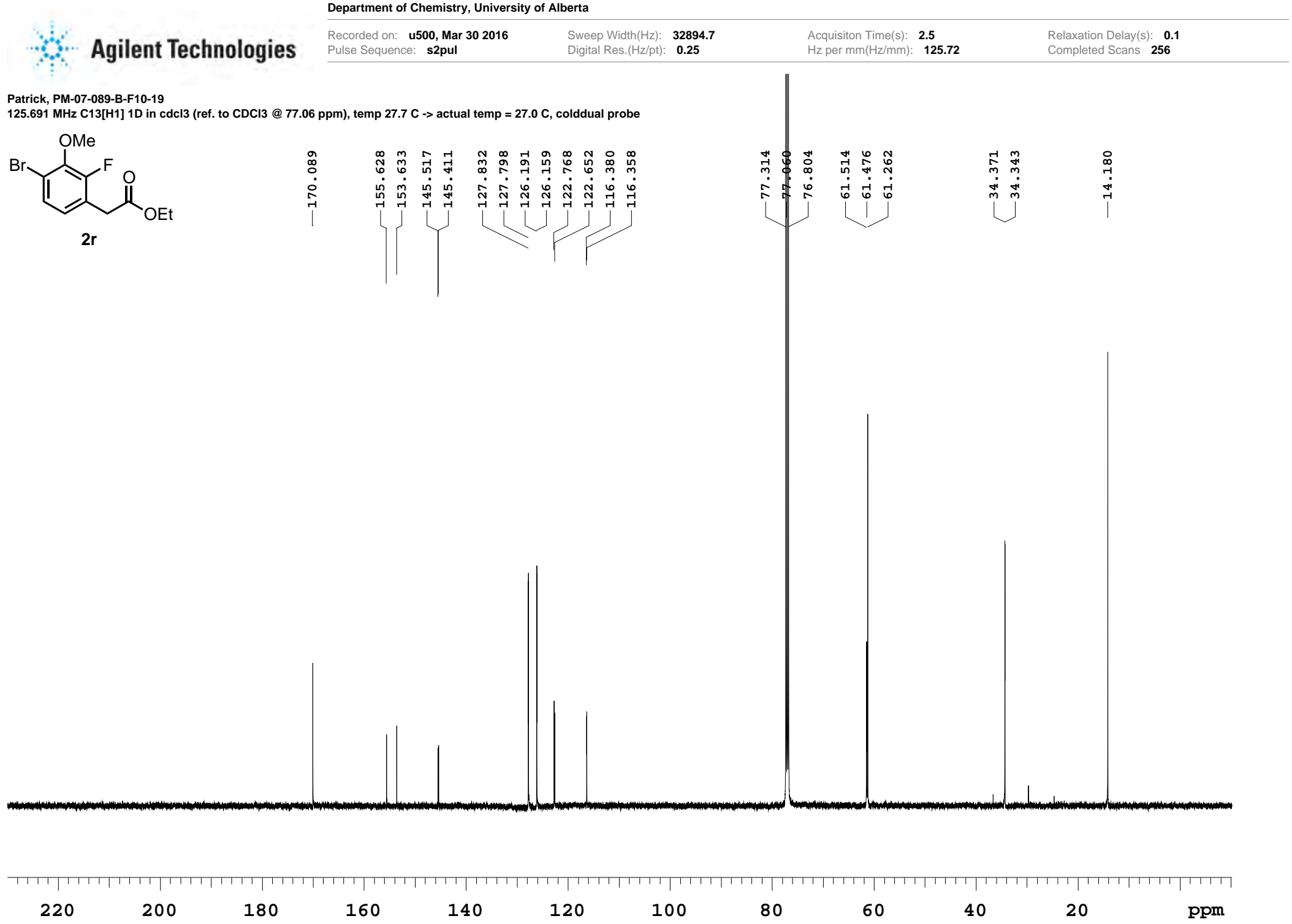

File: /mnt/d600/home13/ijlnmr/nmrdata/DATA_FROM_NMRSERVICE/Patrick/2016.03/2016.03.30.u5_PM-07-089-B-F10-19_loc10_07.47_C13_1D 
Department of Chemistry, University of Alberta

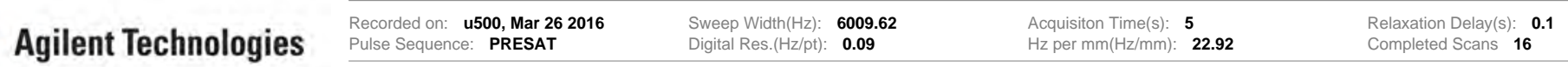

Patrick, PM-07-073-A-F13-14-2

作

OEt

2s

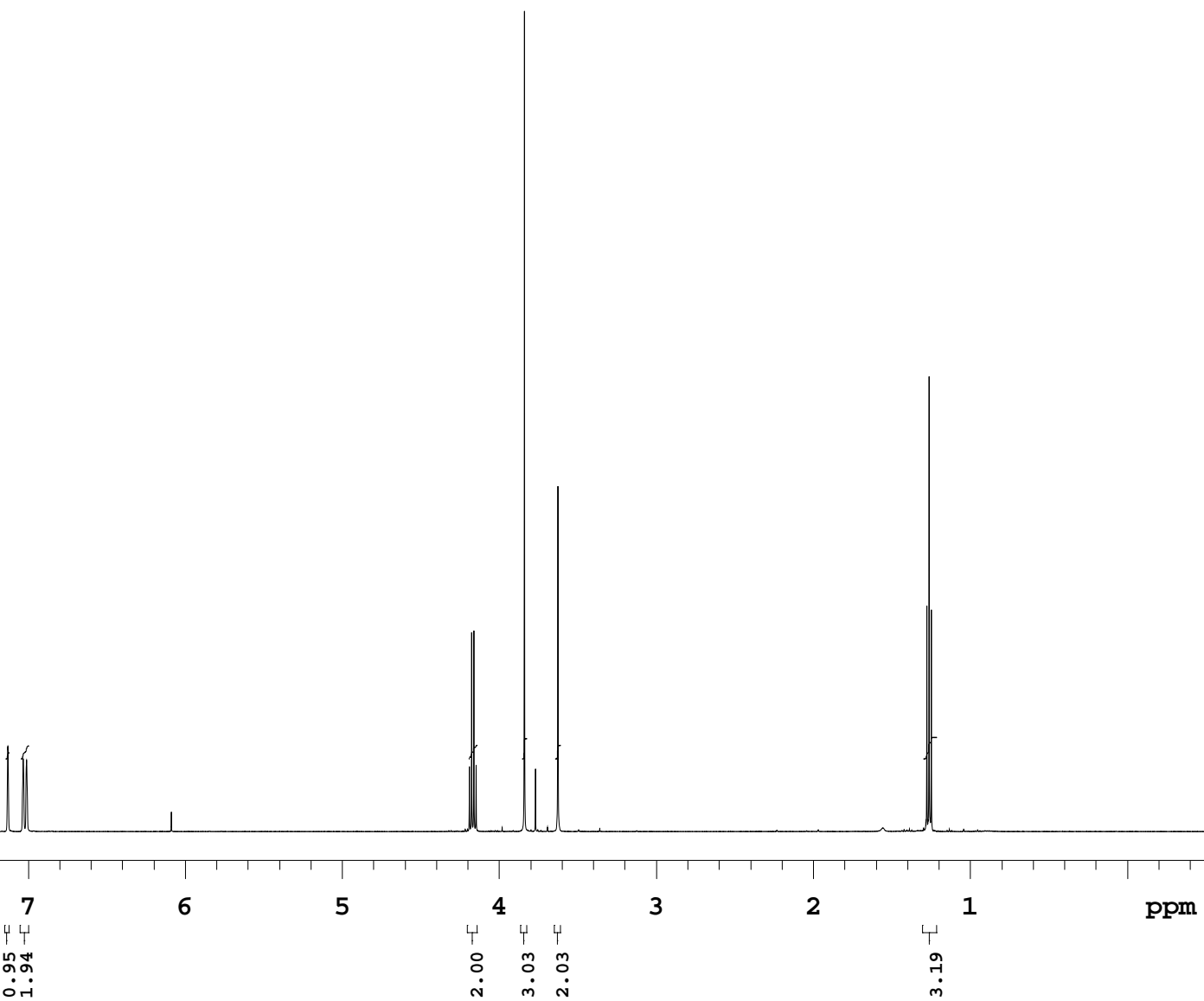

File: /mnt/d600/home13/rijnmr/nmrdata/DATA_FROM_NMRSERVICE/Patrick/2016.03/2016.03.26.u5_PM-07-073-A-F13-14-2_loc8_19.09_H1_1D 
Department of Chemistry, University of Alberta

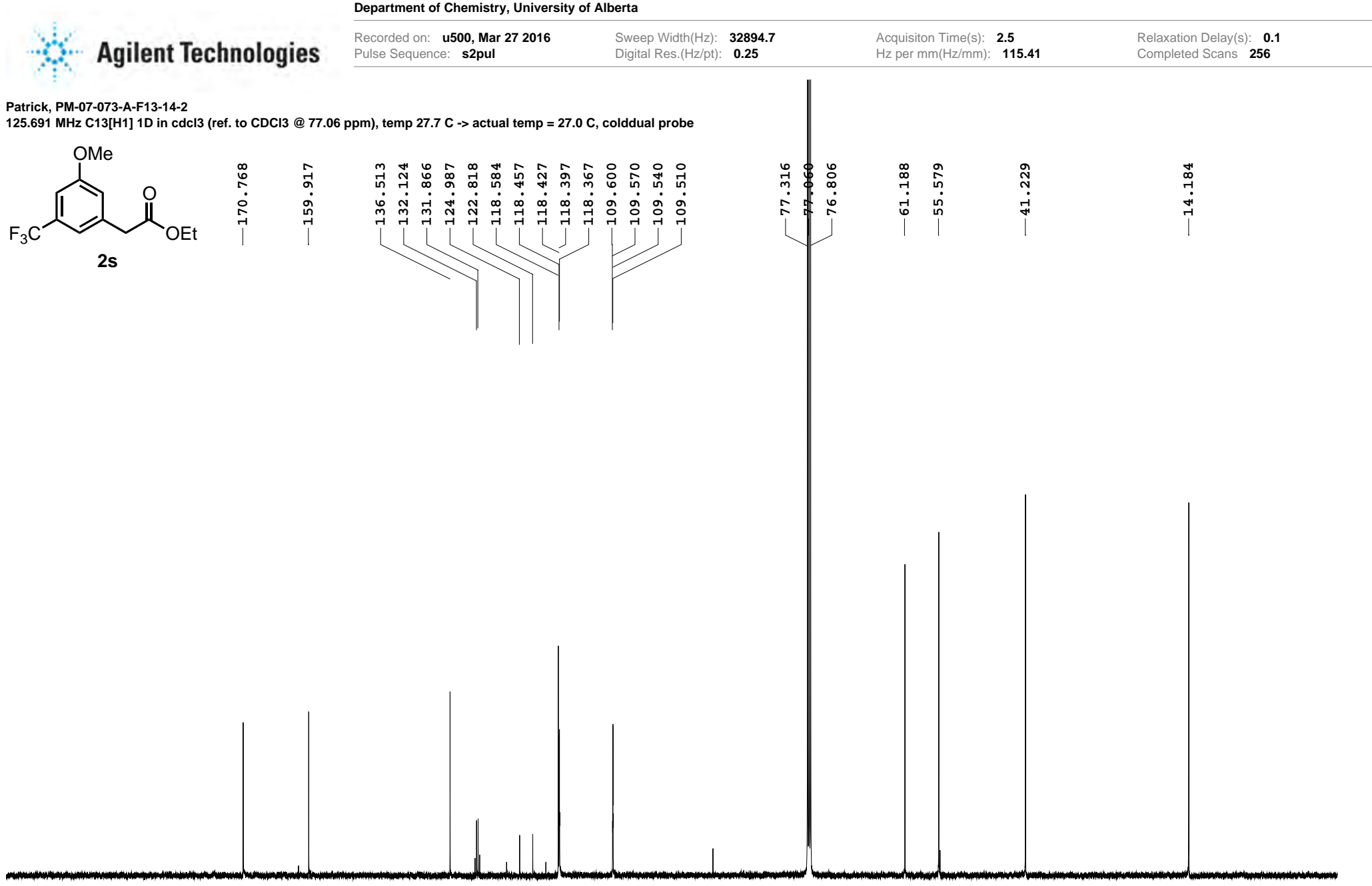


Department of Chemistry, University of Alberta

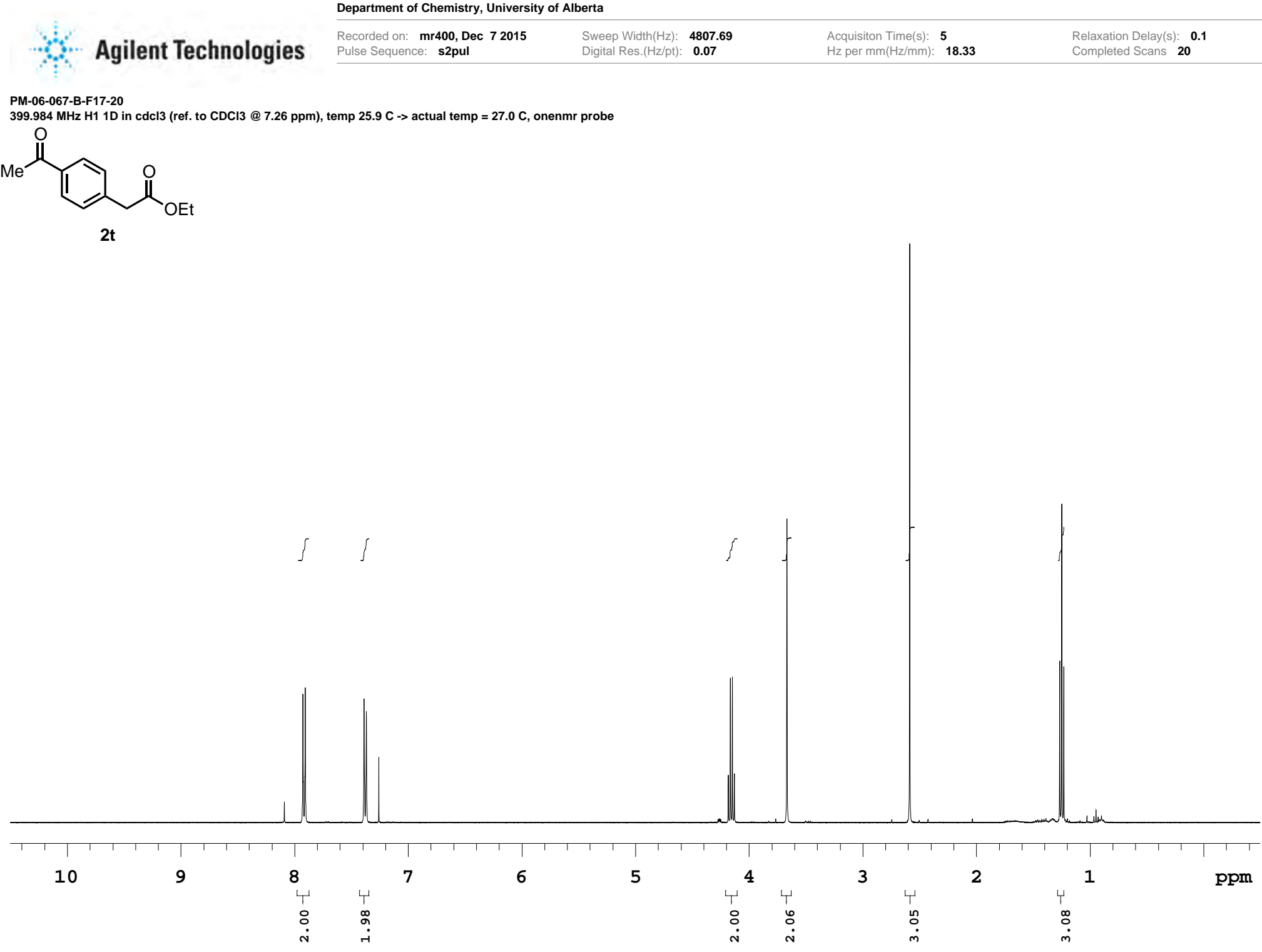

File: /mnt/d600/home13/rilnmr/hmrdata/Patrick/2015.12/2015.12.07.mr4_PM-06-067-B-F17-20_H1_1D 
Department of Chemistry, University of Alberta

\begin{tabular}{|c|c|c|c|c|}
\hline Aailent Technoloaies & $\begin{array}{l}\text { Recorded on: u500, Feb } 122016 \\
\text { Pulse Sequence: PRESAT }\end{array}$ & 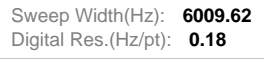 & 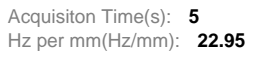 & $\begin{array}{l}\text { Relaxation Delay(s): } \mathbf{0 . 1} \\
\text { Completed Scans } \mathbf{1 6}\end{array}$ \\
\hline
\end{tabular}

Patrick, PM-06-187-F14-16

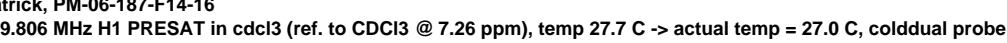

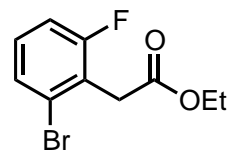

$2 \mathbf{u}$

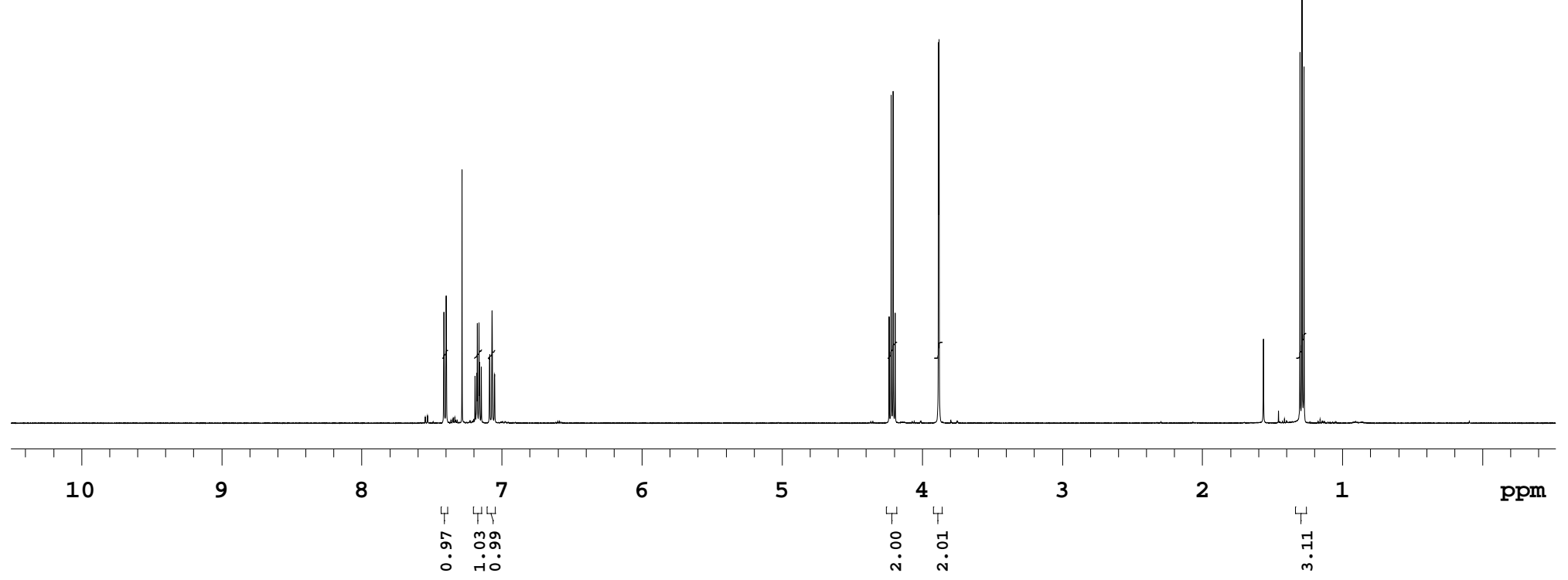

File: /mnt/d600/home13/rilnmr/nmrdata/DATA_FROM_NMRSERVICE/Patrick/2016.02/2016.02.12.u5_PM-06-187-F14-16_loc1_17.26_H1_1D 
Department of Chemistry, University of Alberta

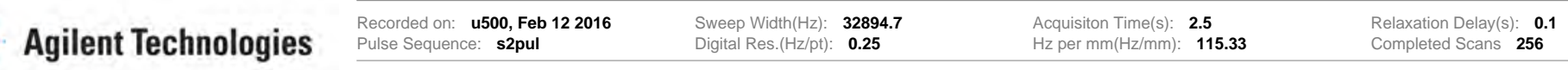

Patrick, PM-06-187-F14-16

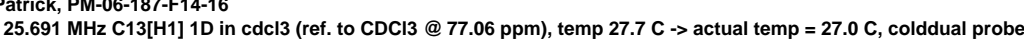

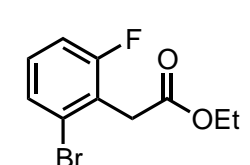

$2 u$

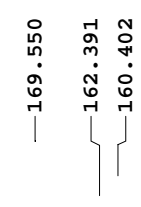

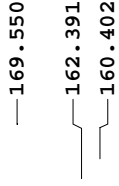

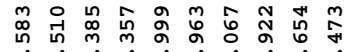

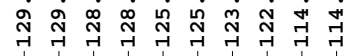

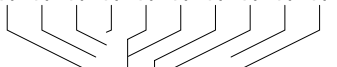

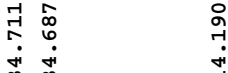

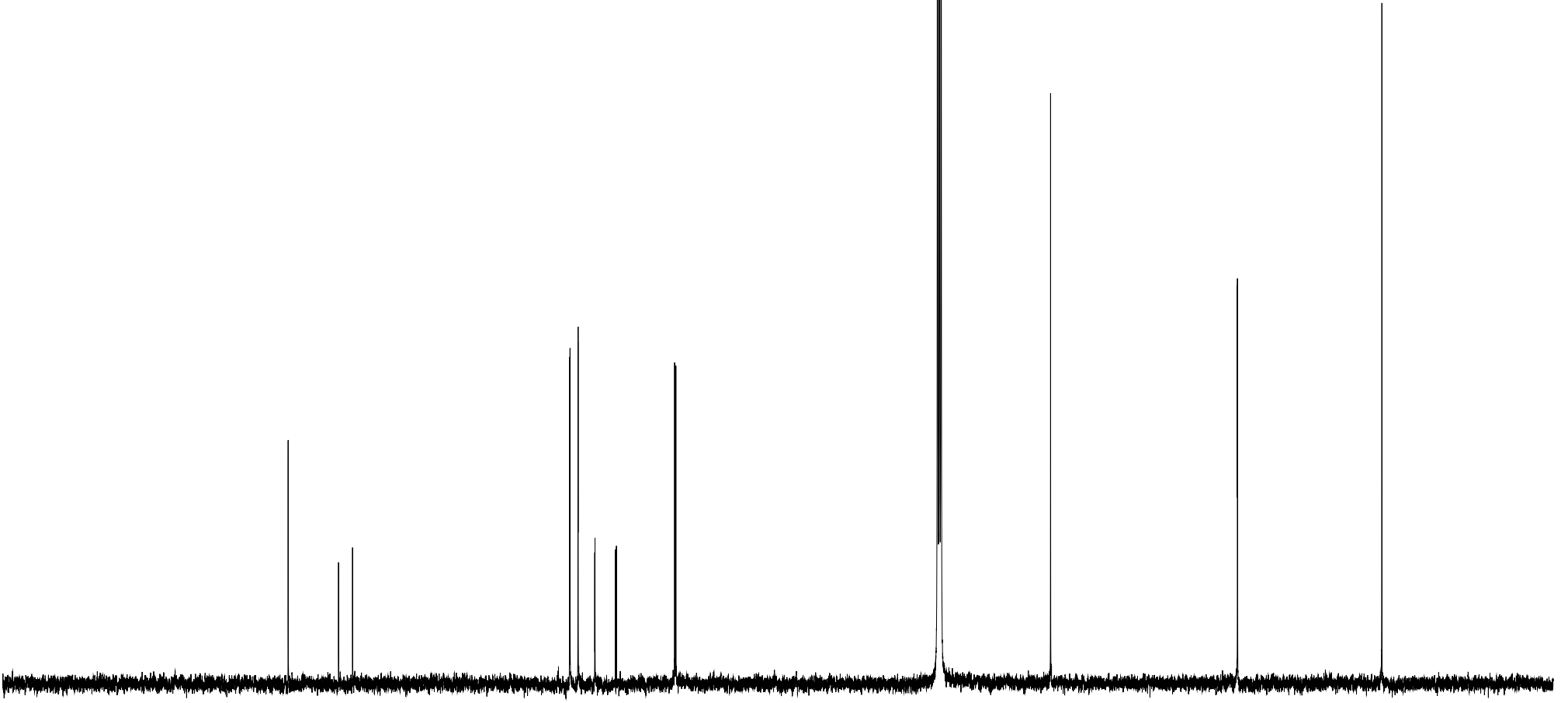

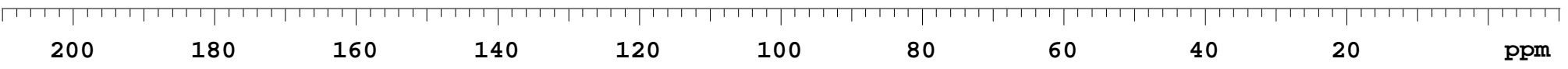


Department of Chemistry, University of Alberta

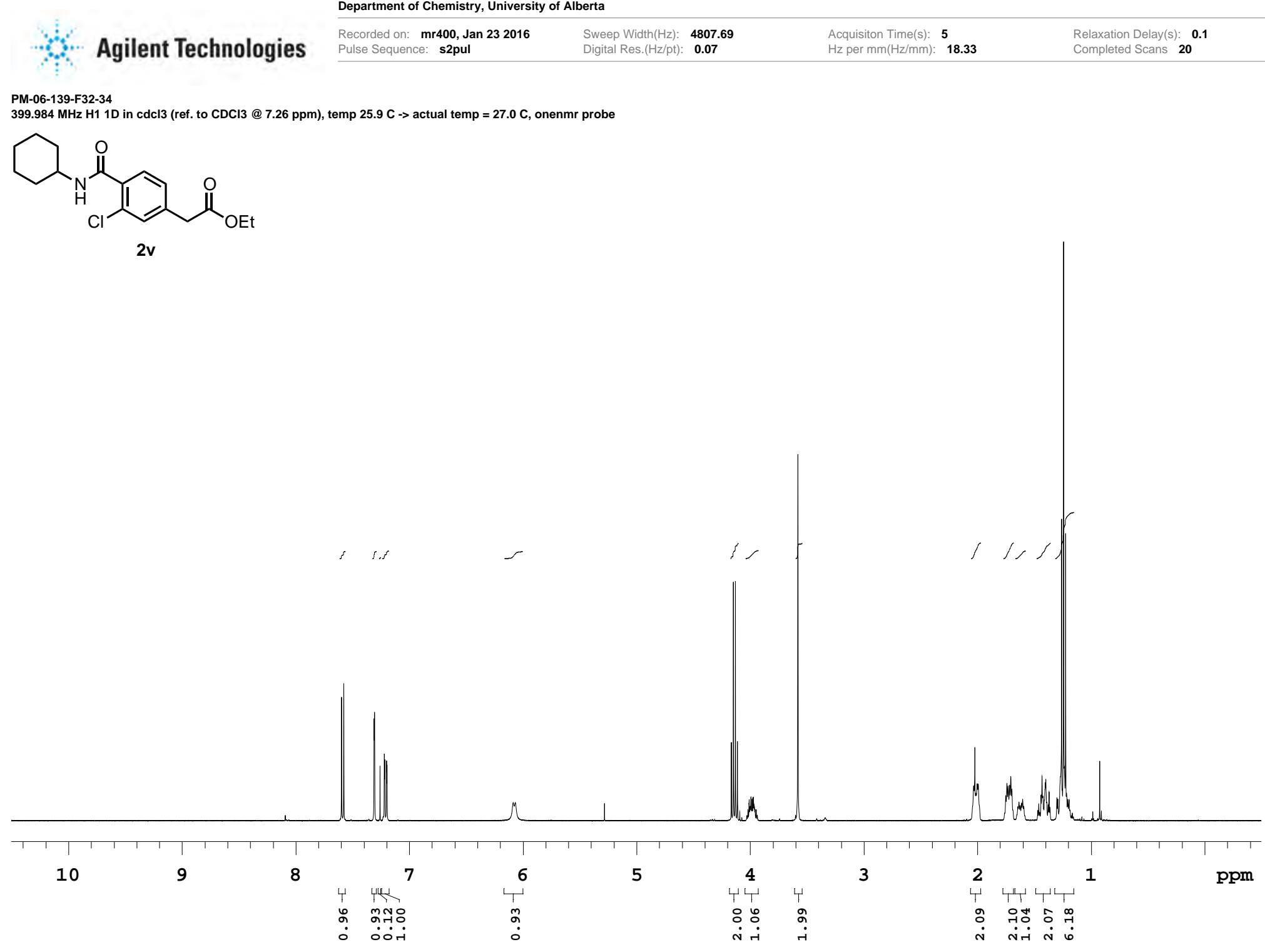

File: /mnt/d600/home13/rilnmr//nmrdata/Patrick/2016.01/2016.01.23.mr4_PM-06-139-F32-34_H1_1D 
Department of Chemistry, University of Alberta

\begin{tabular}{|c|c|c|c|c|}
\hline Anilent Technolo & $\begin{array}{l}\text { Recorded on: mr400, Jan } 232016 \\
\text { Pulse Sequence: s2pul }\end{array}$ & $\begin{array}{l}\text { Sweep Width(Hz): } \quad \mathbf{2 7 1 7 3 . 9} \\
\text { Digital Res.(Hz/pt): } \mathbf{0 . 2 1}\end{array}$ & $\begin{array}{l}\text { Acquisiton Time(s): } \mathbf{2 . 5} \\
\text { Hz per } m m(H z / m m):\end{array}$ & $\begin{array}{l}\text { Relaxation Delay(s): } 0.1 \\
\text { Completed Scans } 292\end{array}$ \\
\hline
\end{tabular}

PM-06-139-F32-34

100.587 MHz C13[H1] 1D in cdc13 (ref. to CDC13 @ $77.06 \mathrm{ppm}$ ), temp $25.9 \mathrm{C}>>$ actual temp $=27.0 \mathrm{C}$, onenmr probe

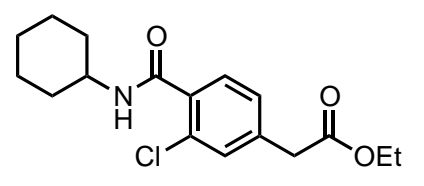

总兽

กิ

2v

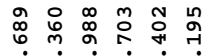

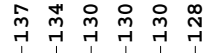

(l)

İ$$
\text { . }
$$
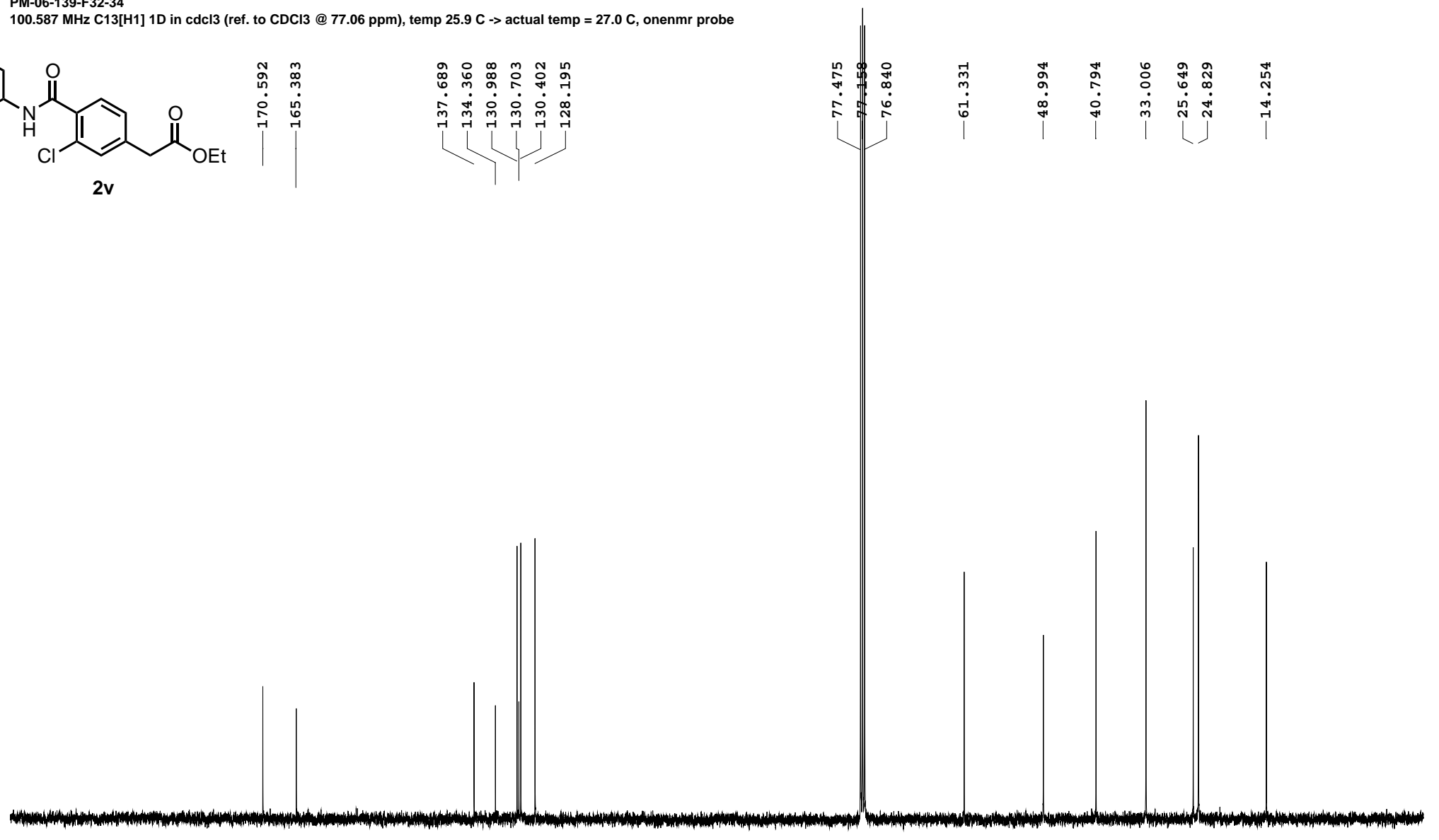

\begin{tabular}{|c|c|c|c|c|c|c|c|c|c|}
\hline 200 & 180 & 160 & 140 & 120 & 100 & 80 & 60 & 40 & 20 \\
\hline
\end{tabular}


Department of Chemistry, University of Alberta

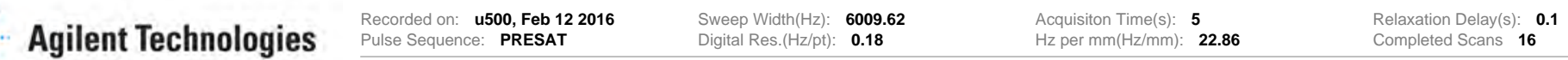

Patrick, PM-06-191-F21-26

作

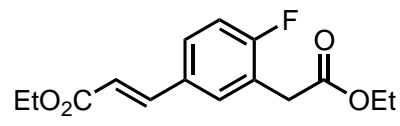

2w

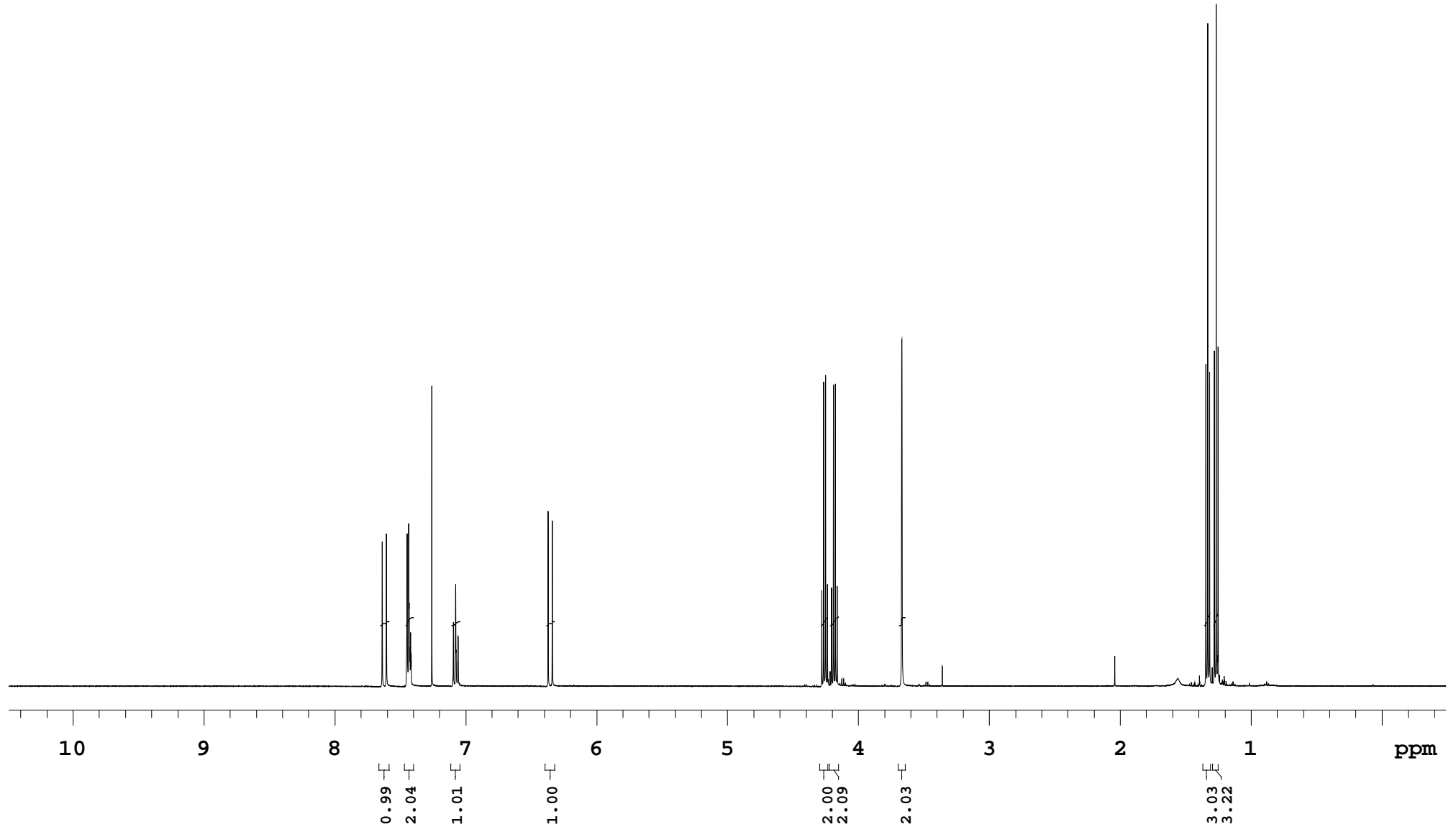

File: /mnt/d600/home13/rijnmr/nmrdata/DATA_FROM_NMRSERVICE/Patrick/2016.02/2016.02.12.u5_PM-06-191-F21-26_loc2_17.43_H1_1D 
Department of Chemistry, University of Alberta

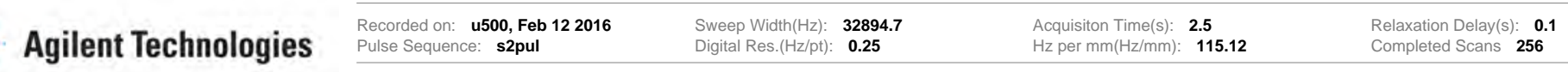

Patrick, PM-06-191-F21-26

125.091 $\mathrm{MH}$ C13[H1] $1 \mathrm{D}$ in $\mathrm{cdcl} 3$ (ref. to $\mathrm{CDCl} 3 @ 77.06 \mathrm{ppm}$ ), temp $27.7 \mathrm{C} \rightarrow$ actual temp $=27.0 \mathrm{C}$, colddual probe

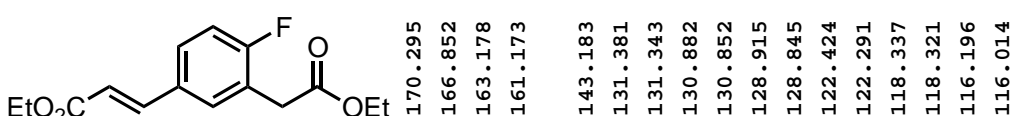

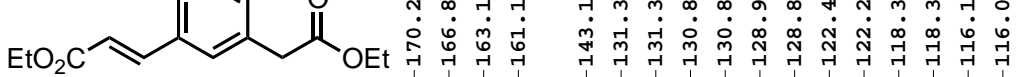
2w

(1)

(1)
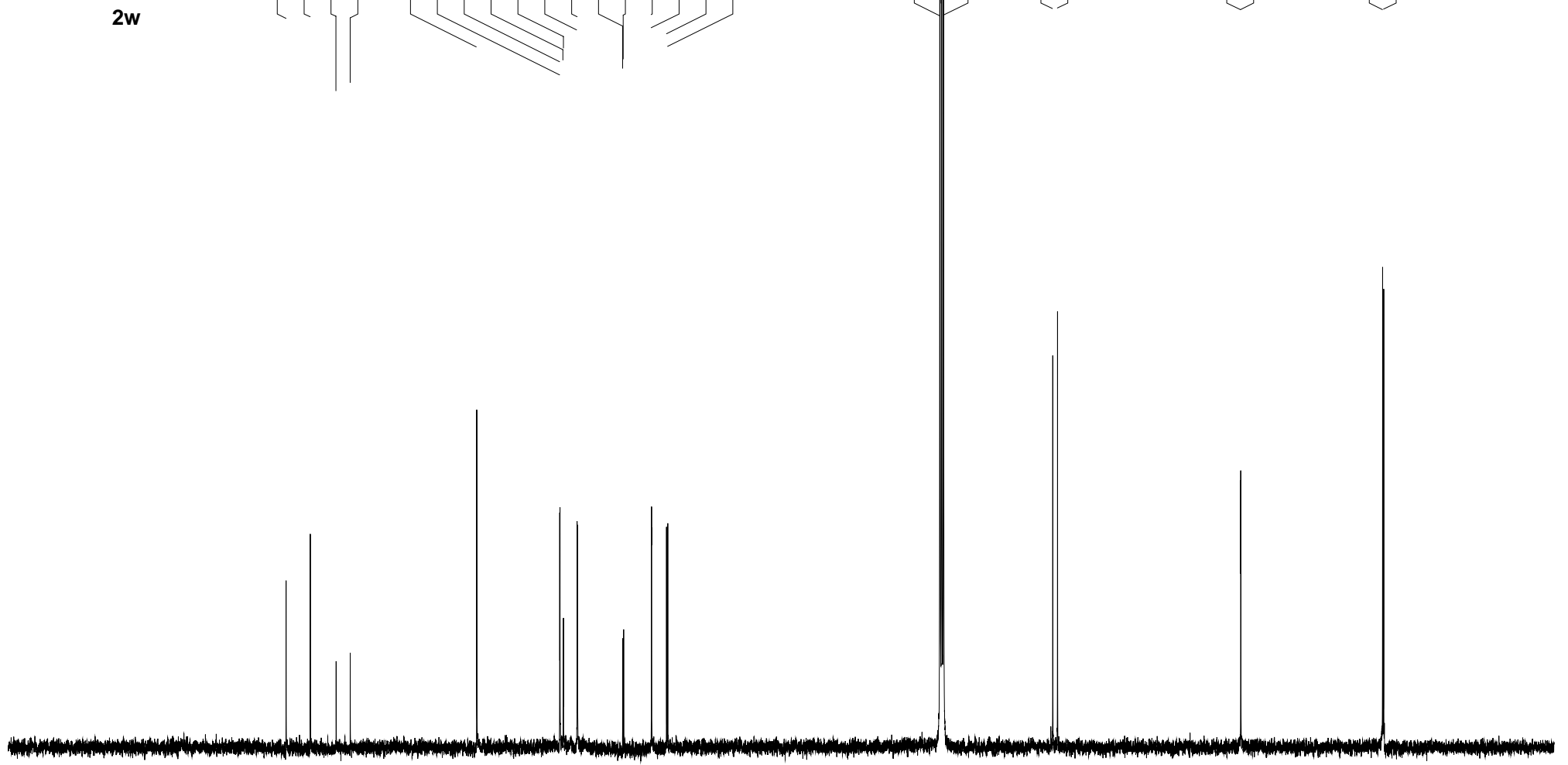

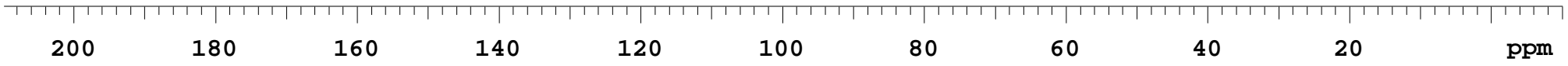


Department of Chemistry, University of Alberta

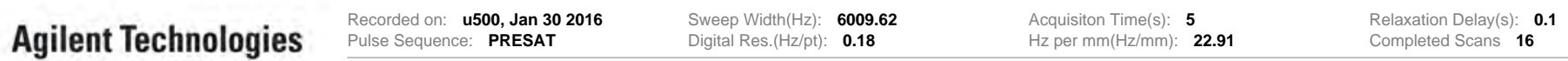

Patrick, PM-06-161-F18-22

(209.806 MHz H1 PRESAT in cdcl3 (ref. to CDC13 @ 7.26 ppm), temp $27.7 \mathrm{C} \rightarrow$ actual temp = 27.0 C, colddual probe

$\mathrm{Cl}$
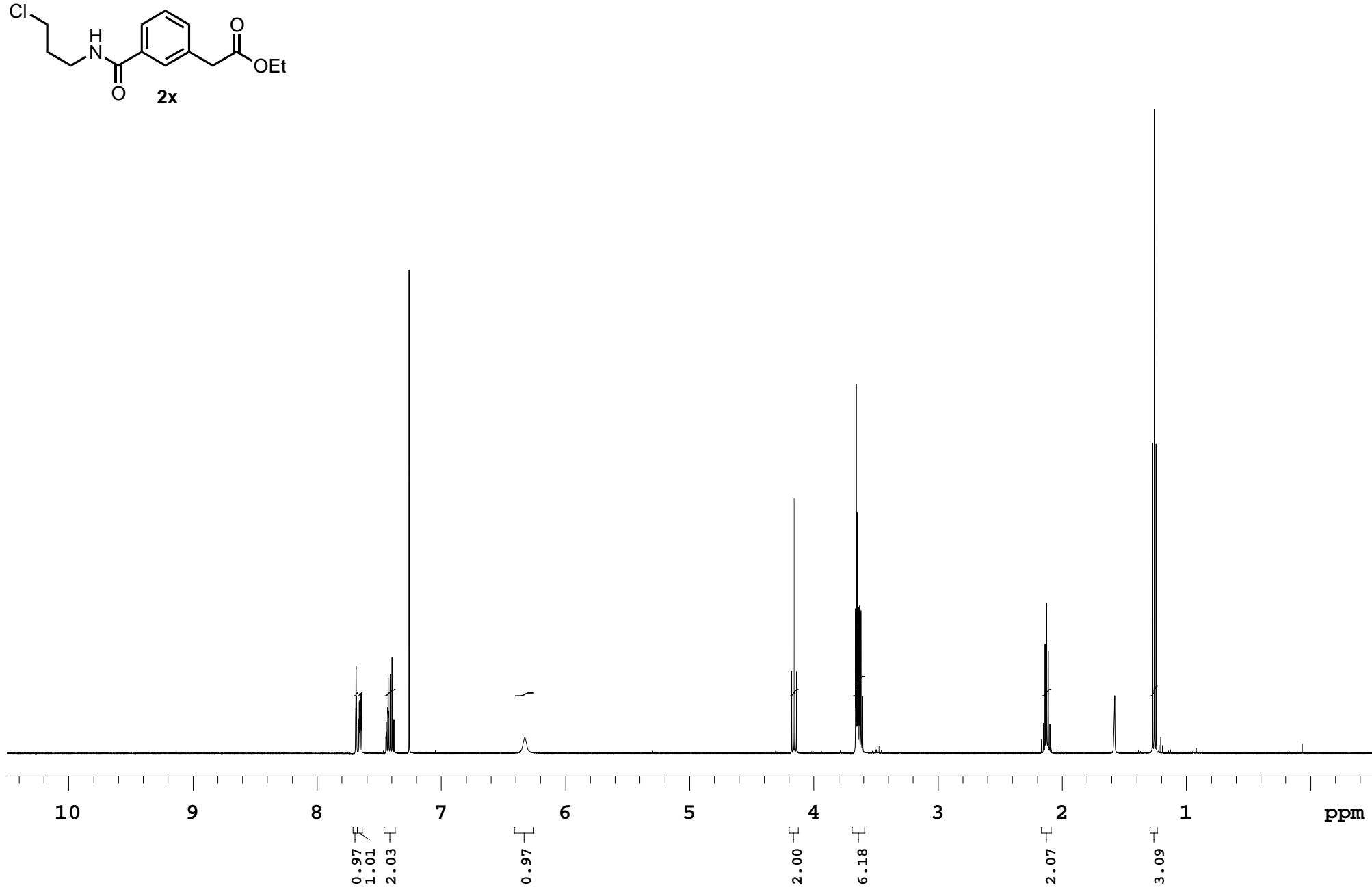

File: /mnt/d600/home13/rilnmr/nmrdata/DATA_FROM_NMRSERVICE/Patrick/2016.01/2016.01.30.u5_PM-06-161-F18-22_loc9_11.38_H1_1D 
Department of Chemistry, University of Alberta

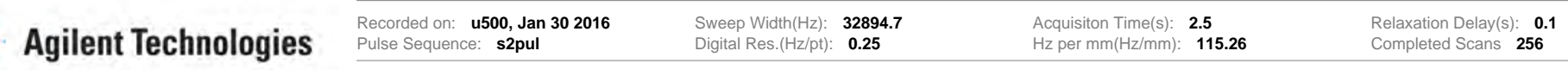

Patrick, PM-06-161-F18-22
$125.691 \mathrm{MHz}$ C13[H1] $1 \mathrm{D}$ in cdcl3 (ref. to CDCl3 @ $77.06 \mathrm{ppm}$ ), temp $27.7 \mathrm{C} \rightarrow$ actual temp = 27.0 C, colddual probe

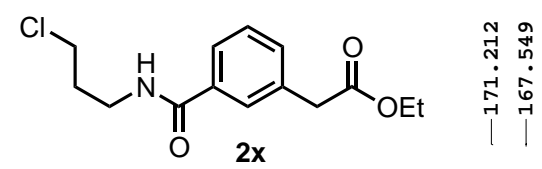

密获哭品 :

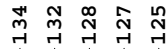

సิ

i

$\lfloor\lfloor\lfloor\rfloor]$

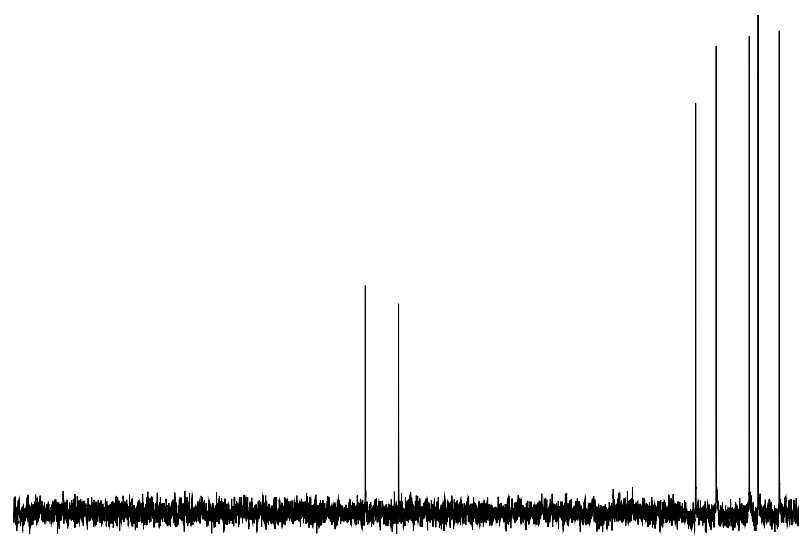

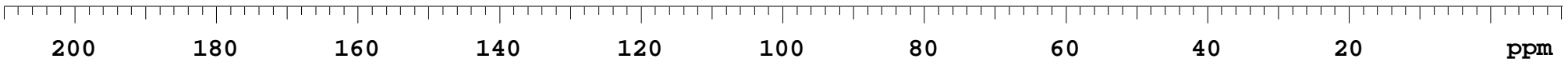


Department of Chemistry, University of Alberta

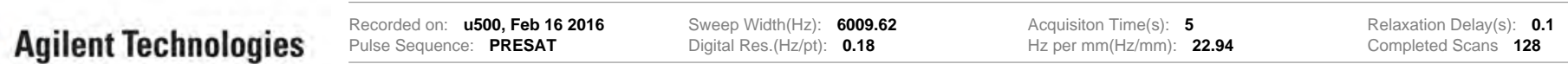

Patrick, PM-06-199-B-C2-F27-31

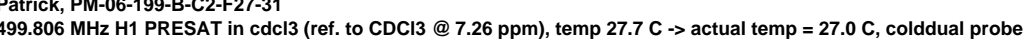

$\mathrm{HO}$,

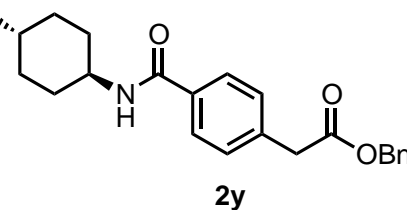

2y

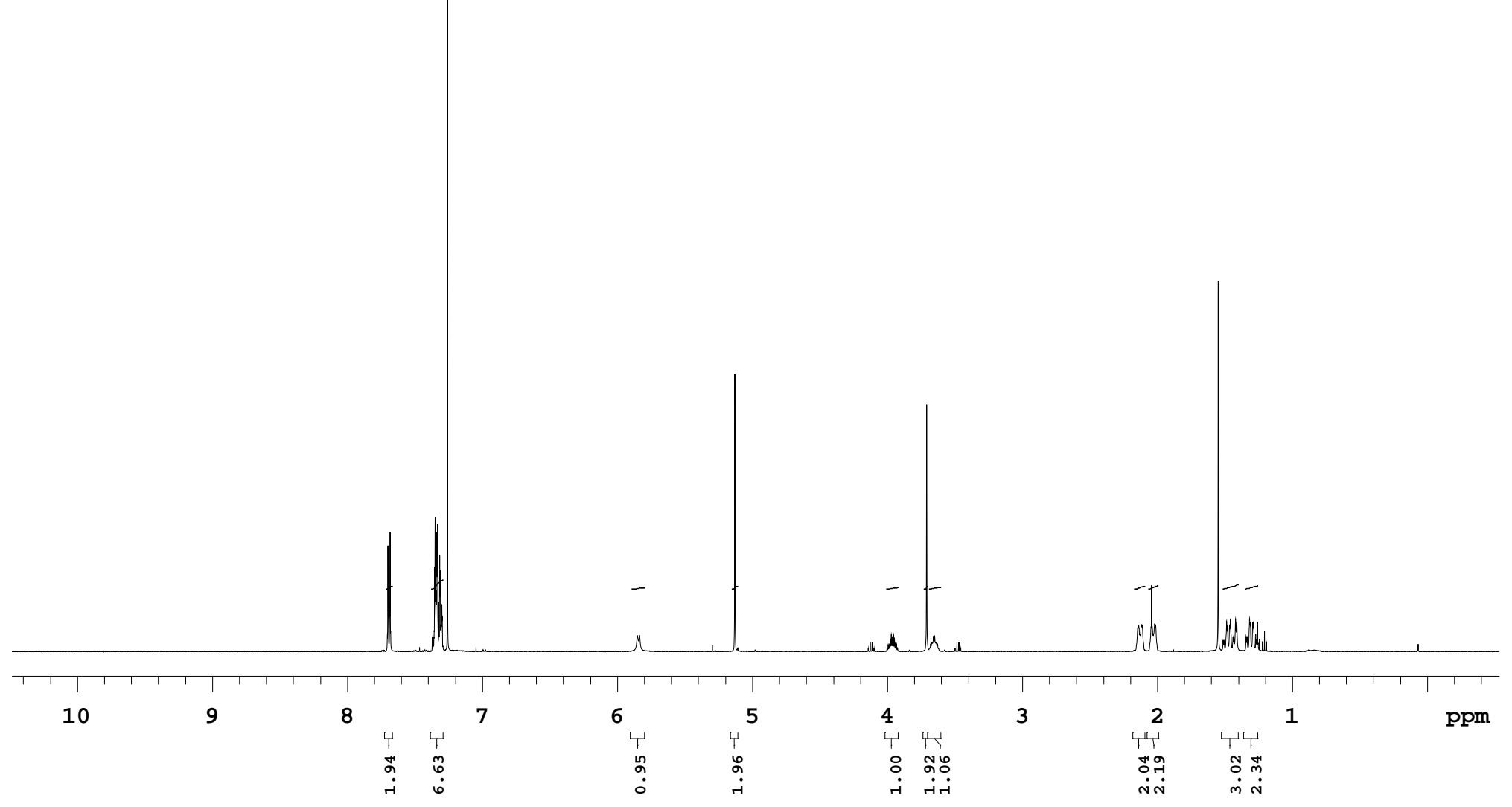

File: /mnt/d600/home13/rilnmr//mmrdata/DATA_FROM_NMRSERVICE/Patrick/2016.02/2016.02.16.u5_PM-06-199-B-C2-F27-31_loc11_00.50_H1_1D 
Department of Chemistry, University of Alberta

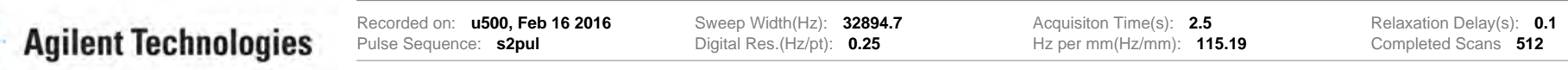

Patrick, PM-06-199-B-C2-F27-31

125.091

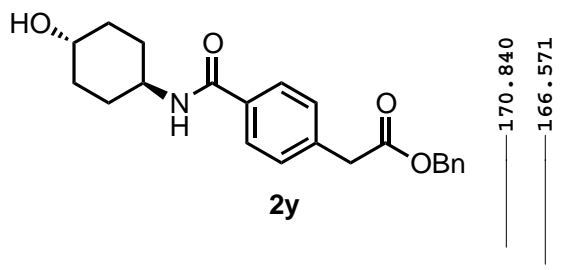

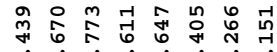

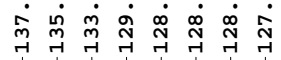

१

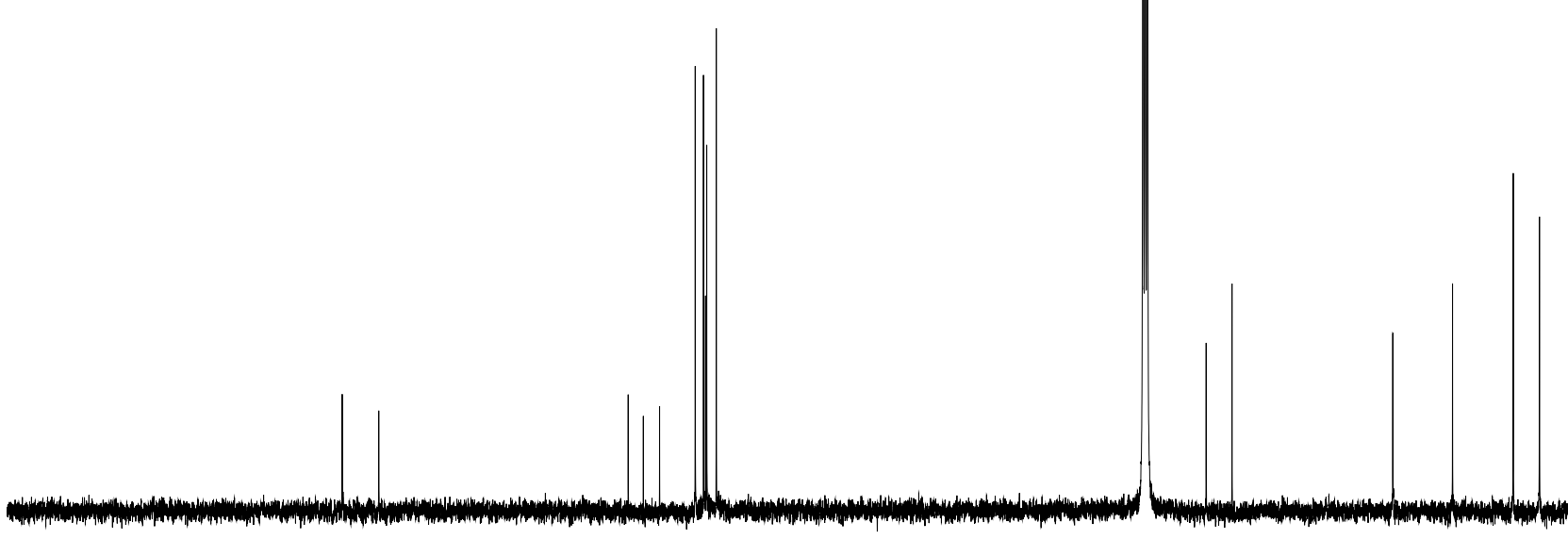

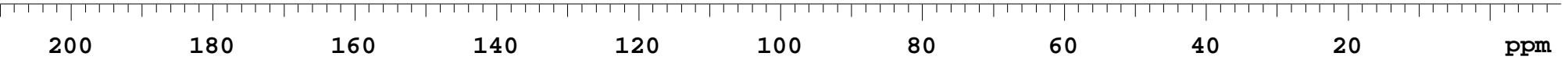


Department of Chemistry, University of Alberta

Agilent Technologies

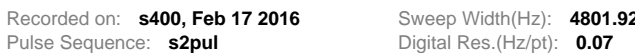

Acquisiton Time(s): 5

$\mathrm{z}$ per $\mathrm{mm}(\mathrm{Hz} / \mathrm{mm}): \mathbf{1 8 . 3 5}$

Relaxation Delay(s): 0.1
Completed Scans 16

Patrick, PM-06-189-F22-24-2

999.949 MHz H1 1D in dmso

1126C $\rightarrow \rightarrow$ actual temp $=120.0$, sw 400 probe

$\prod_{O}$

$2 z$

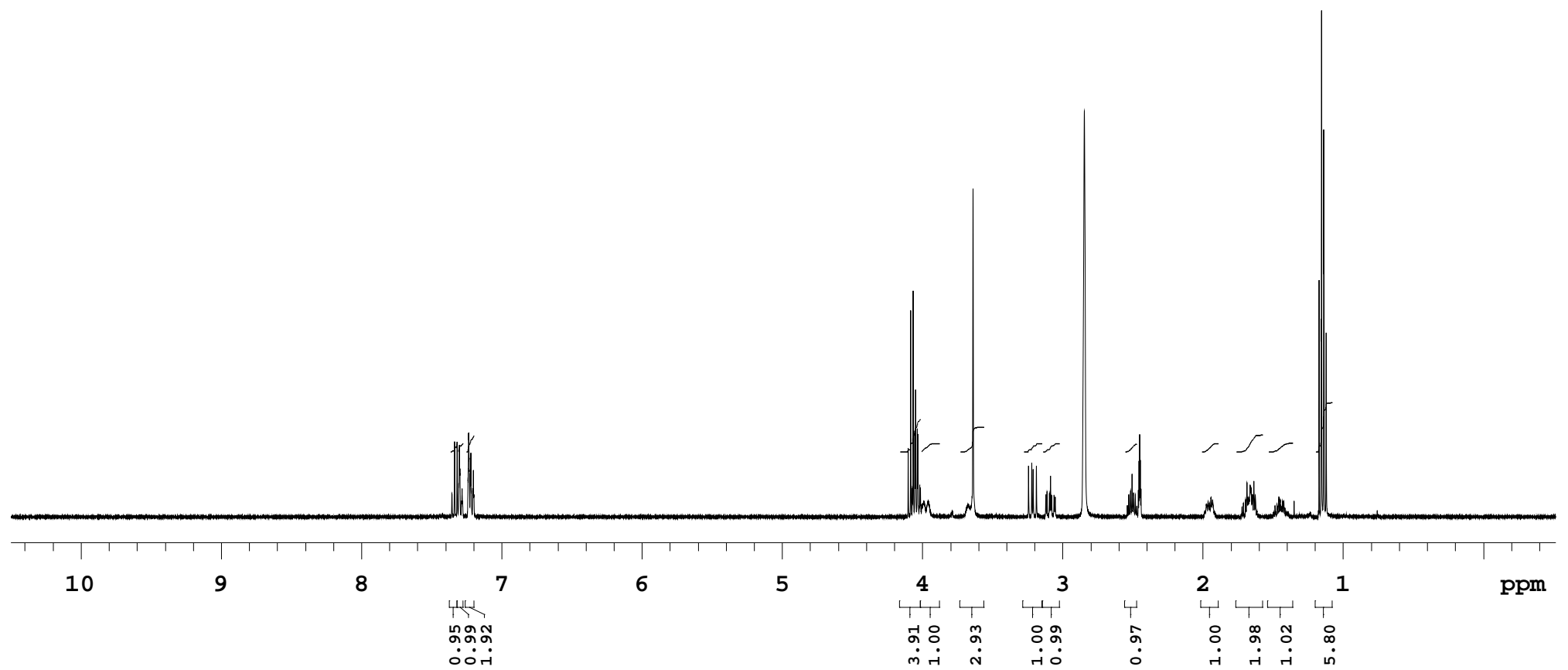

File: /mnt/d600/home13/rinmmr/nmrdata/DATA_FROM_NMRSERVICE/Patrick/2016.02/2016.02.17.s4_PM-06-189-F22-24-2_dmso_120C_H1_1D 
Department of Chemistry, University of Alberta

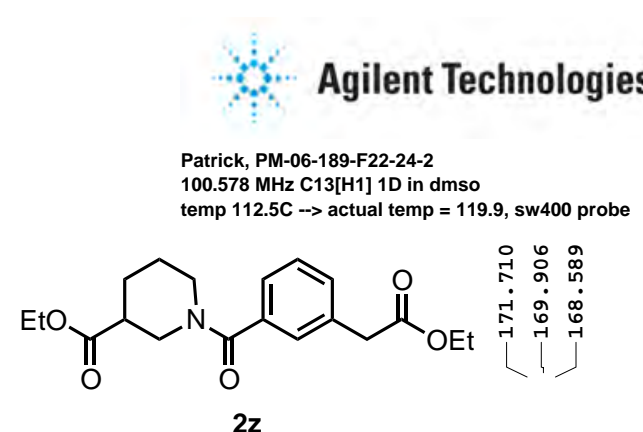

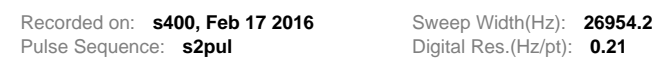
Acquisiton Time(s): 2 Relaxation Delay(s): 0.1

$2 z$

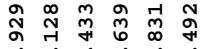

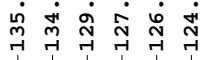

LLLJ

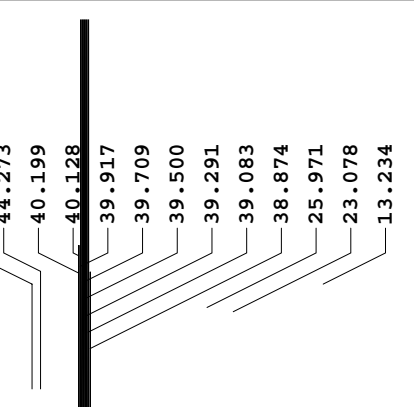

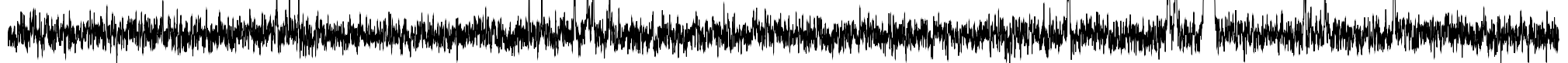

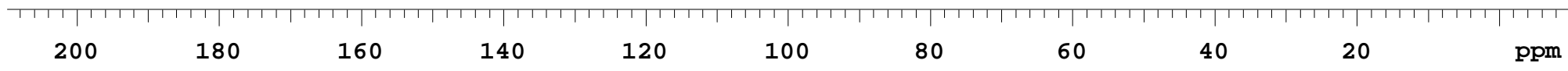




\section{Agilent Technologies \\ Recopided oin v700, Mar 72016

Shengkang, SY-03-81-3

$699.762 \mathrm{MHz}$ H1 PRESAT in edcl3 (ret. to CDC13 @ $7.26 \mathrm{ppm}$ ), temp $27.5 \mathrm{C} \rightarrow$ actual temp $=27.0 \mathrm{C}$, ooldid probe

${ }^{\mathrm{MeO}} \underbrace{\mathrm{N}}_{\mathrm{OBn}}$

$3 a$

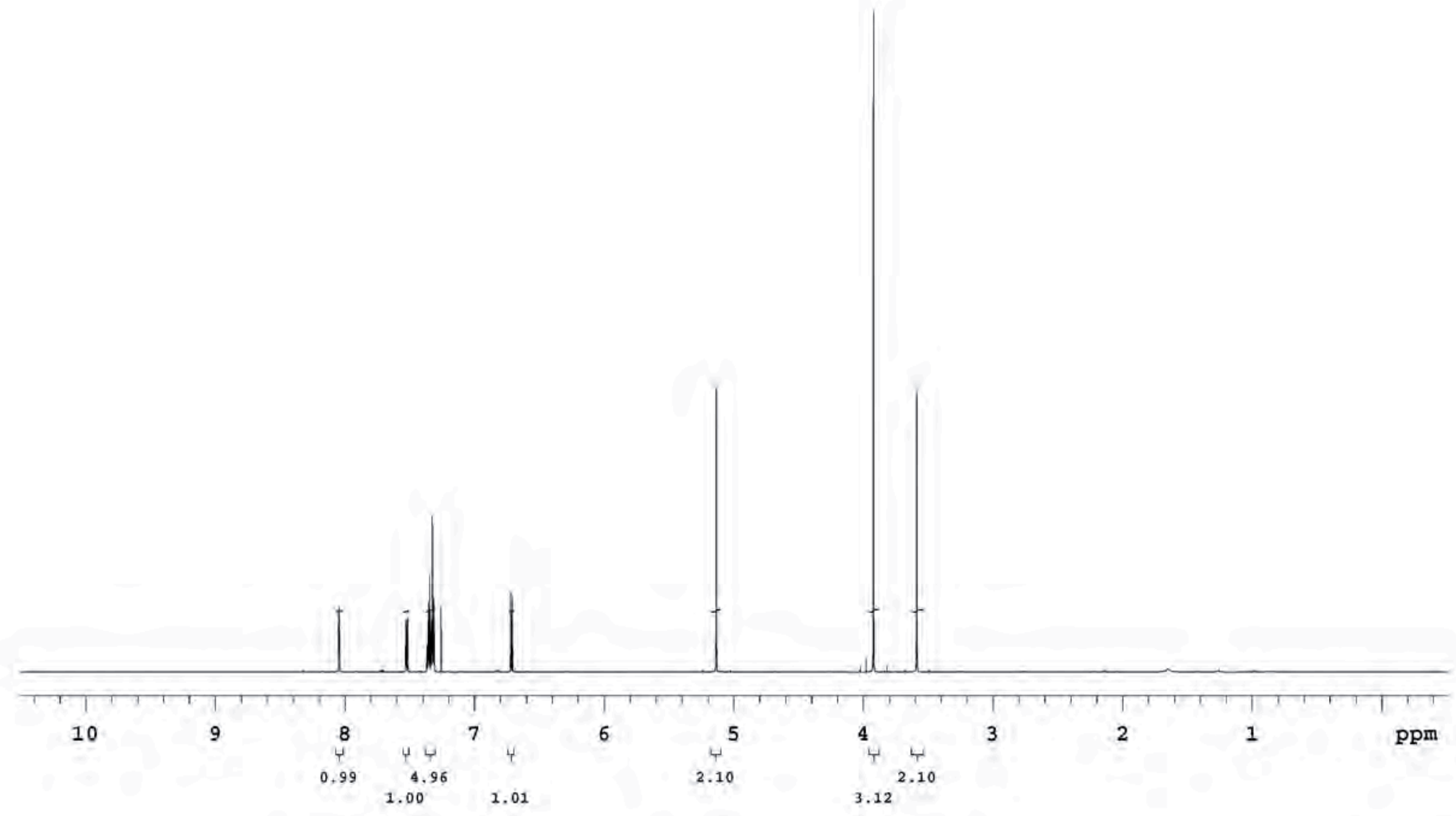


Department of Chemistry, University of Alberta

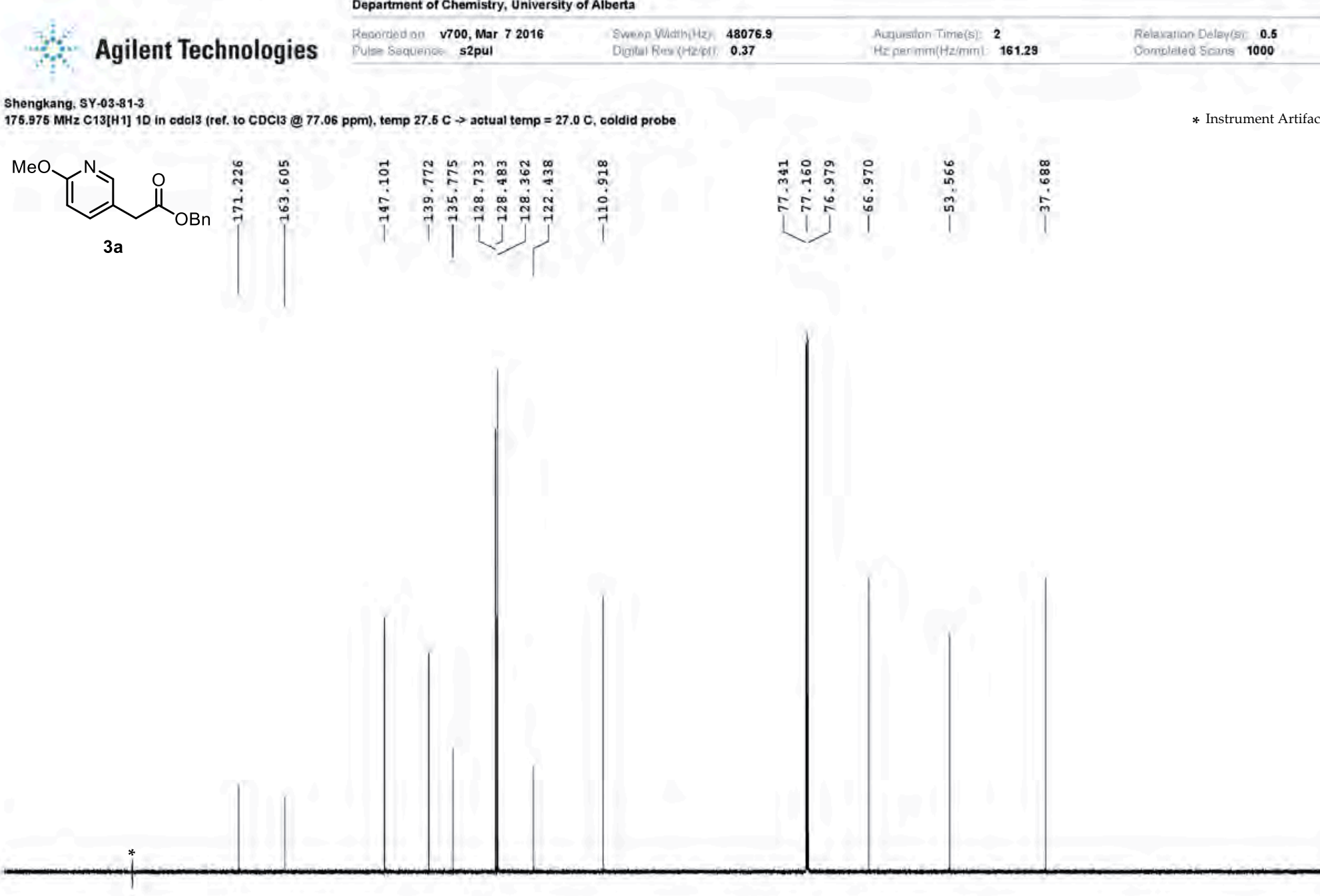
200
180
160
140
120
100
80
60
40
20
ppm 
Department of Chemistry, University of Alberta

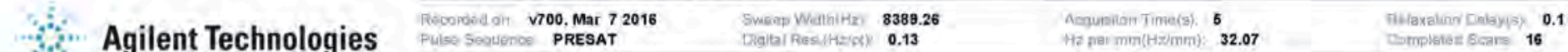

Shengkang, SY-03-81-2
699.762 MHz H1 PRESAT in cdel3 (ret. to CDC13 @ 7.26 ppm), temp $27.5 \mathrm{C} \rightarrow$ actual temp $=27.0 \mathrm{C}$, ooldid probe

$\overbrace{\mathrm{OEt}}^{\mathrm{N}}$

$3 \mathbf{b}$

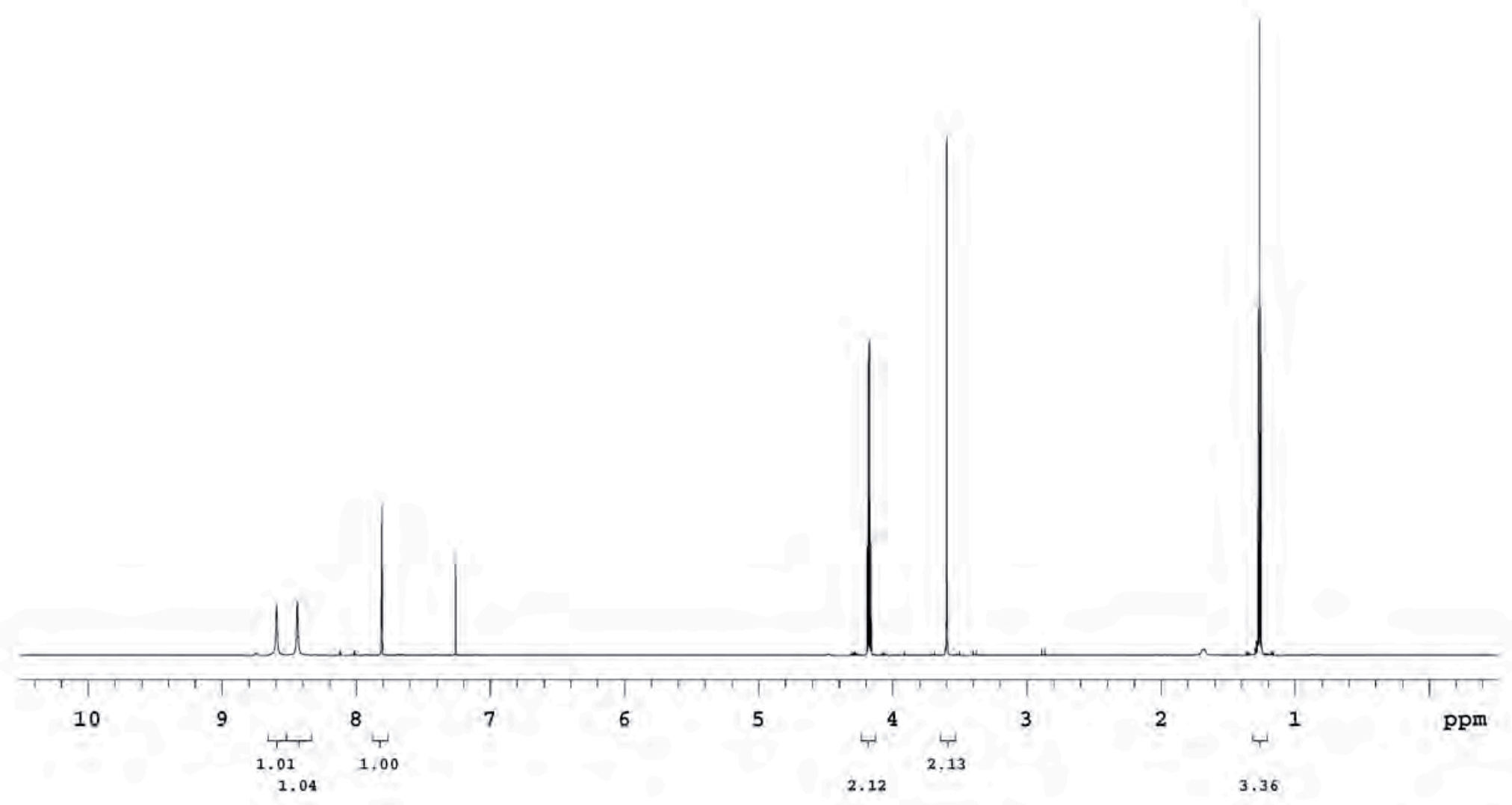


Department of Chemistry, University of Alberta

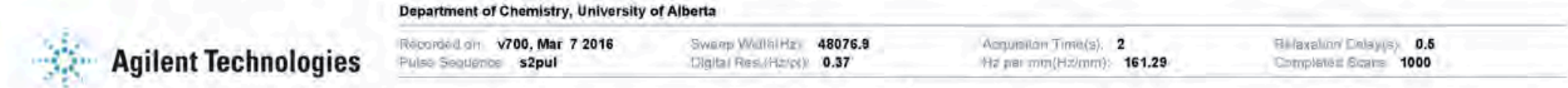
Shengkang, SY-03-81-2 175.975 $\mathrm{MHz} \mathrm{C} 13[\mathrm{H} 1$ 1] $1 \mathrm{D}$ in cdel3 (ref. to $\mathrm{CDCl}$ (e) $77.06 \mathrm{ppm}$ ), temp $27.6 \mathrm{C} \rightarrow$ actual temp $=27.0 \mathrm{C}$, coldid probe

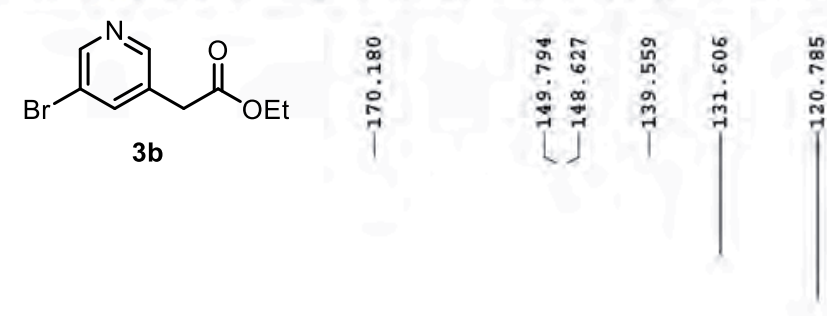

* Instrument Artifact
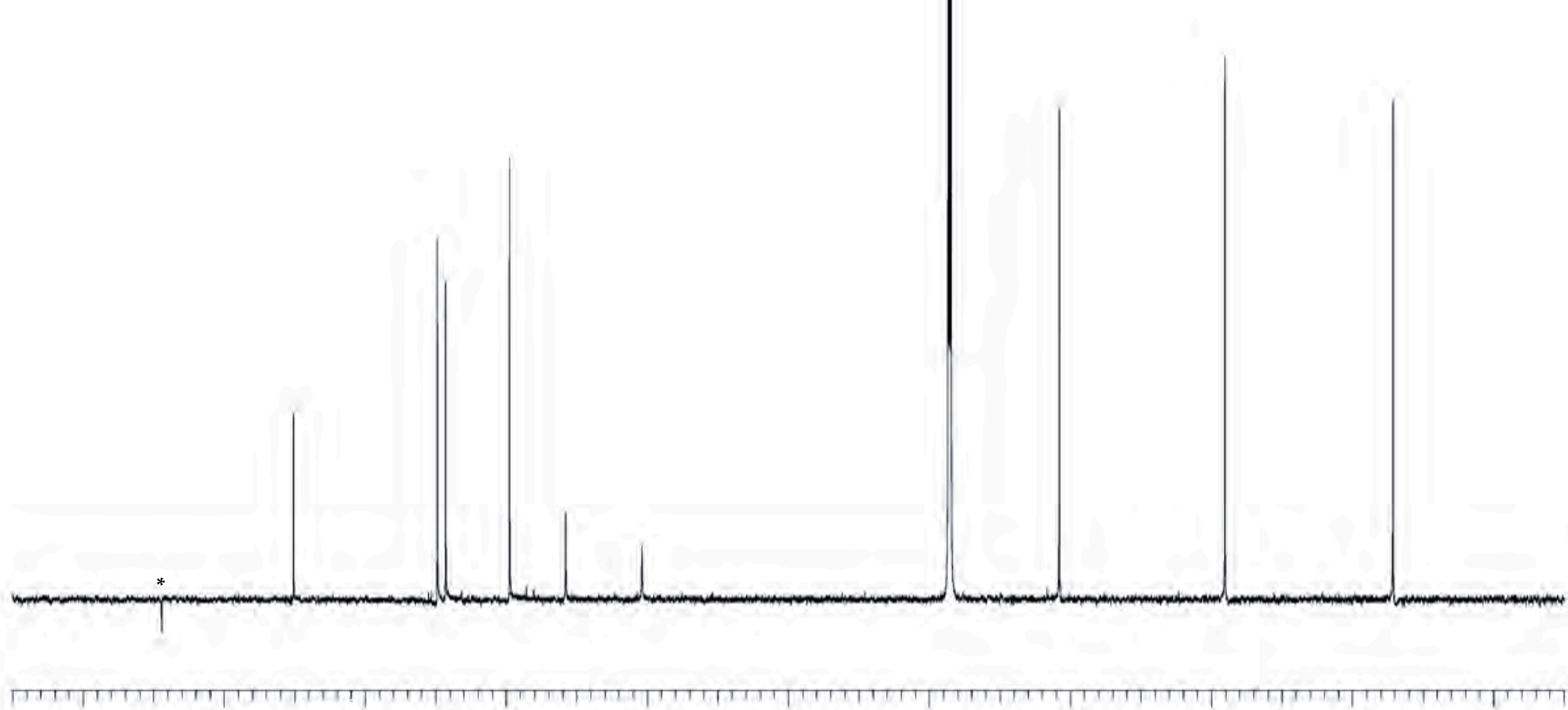

200

180

160

140

120

100

80

60

40

20

ppm 


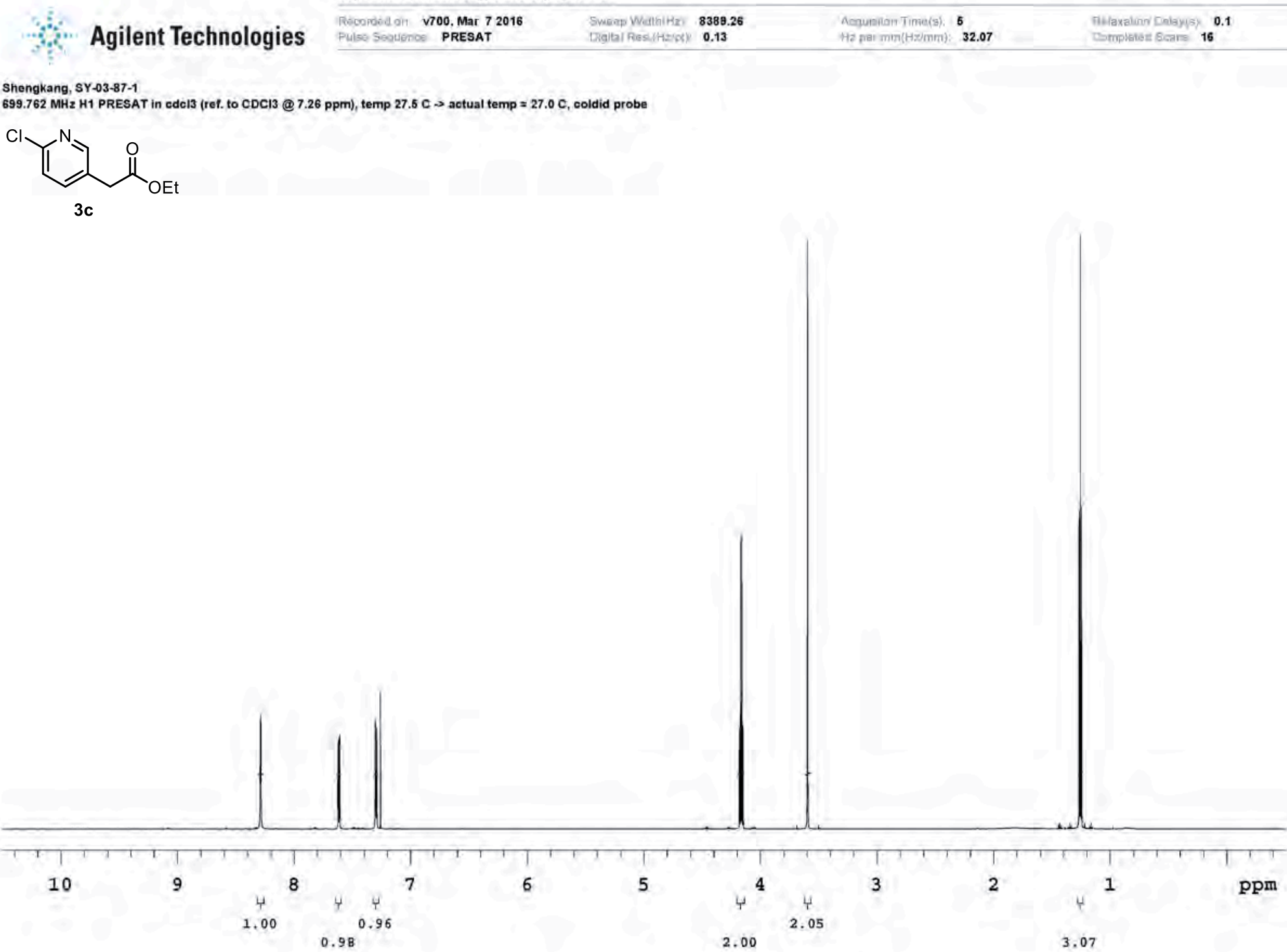


Department of Chemistry, University of Alberta

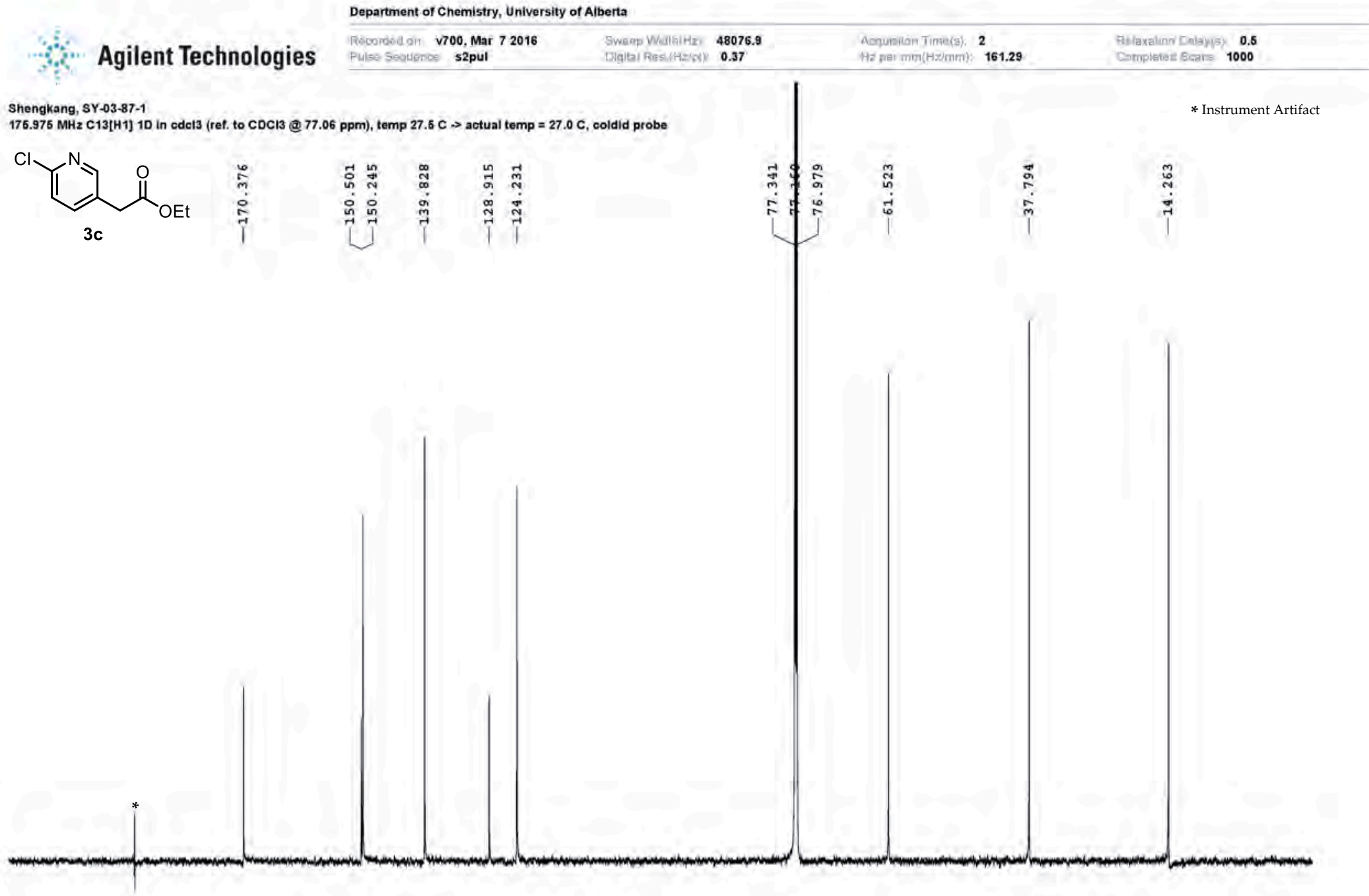


Department of Chemistry, University of Alberta

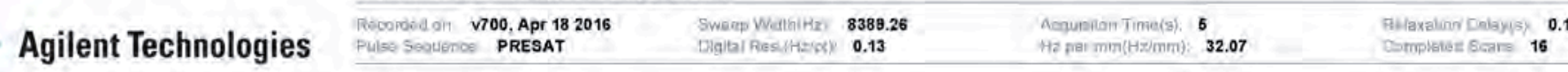

Shengkang, sY-03-183-dry

$699.762 \mathrm{MHz}$ H1 PRESAT in edol3 (ret. to CDCl3 @ $7.26 \mathrm{ppm}$ ), temp $27.5 \mathrm{C} \sim$ actual temp $=27.0 \mathrm{C}$, ooldid probe

${ }^{\mathrm{F}_{3} \mathrm{C}} \underbrace{\mathrm{N}}_{\mathrm{OEt}}$

3d

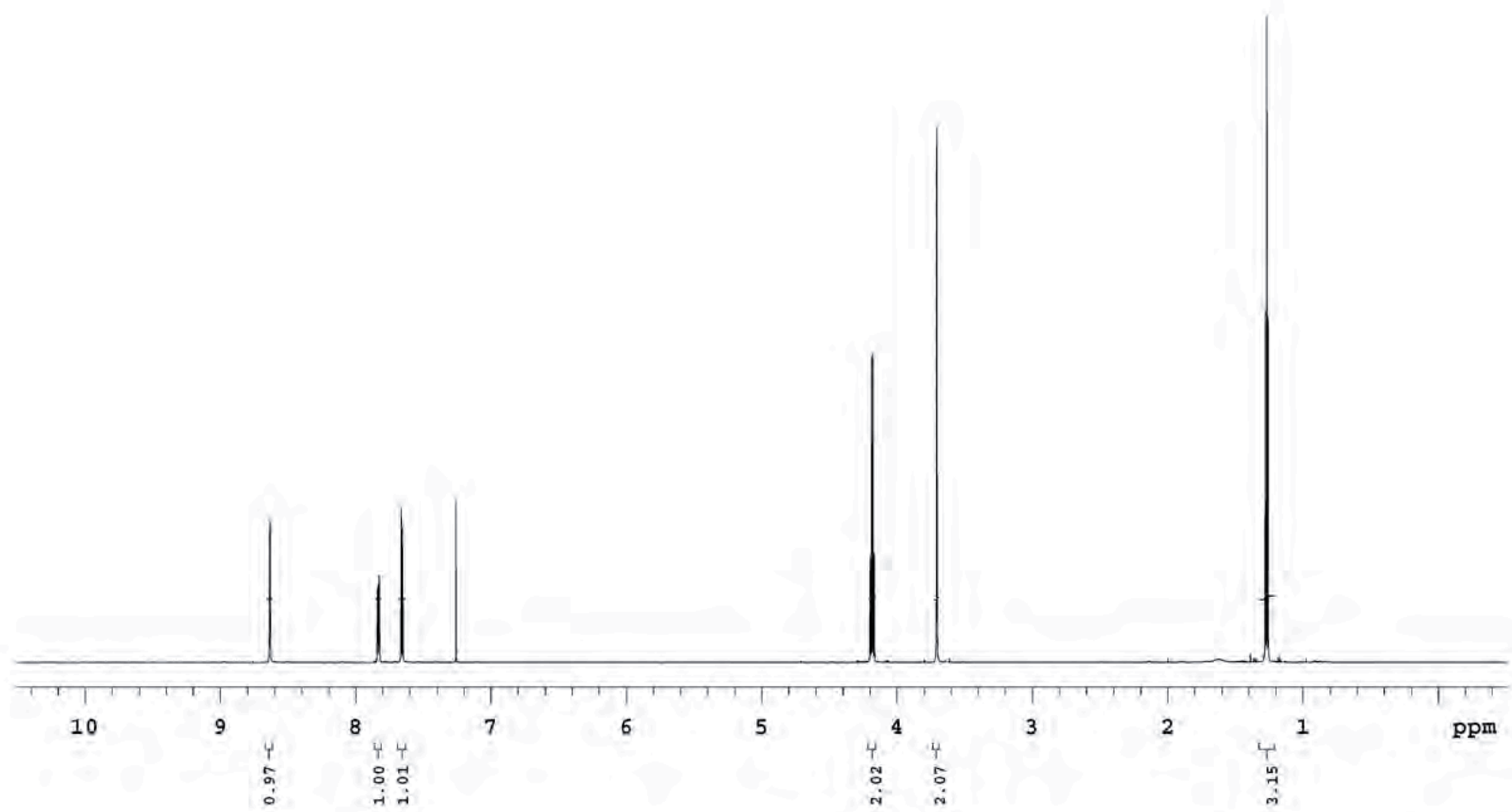


Department of Chemistry, University of Alberta

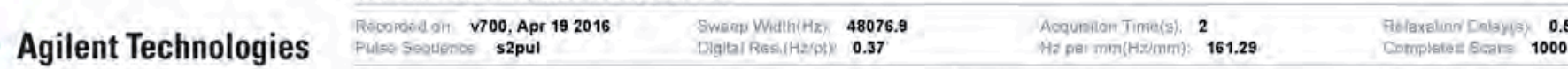
shengkang, SY-03-183 $176.975 \mathrm{MHz} \mathrm{C} 13$ [H1] 10 in cdel3 (ret: to $\mathrm{CDC} 13$ @ $77.06 \mathrm{ppm}$ ), temp $27.6 \mathrm{C} \rightarrow$ actual temp $=27.0 \mathrm{C}$, coldid probe

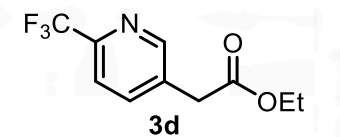

:

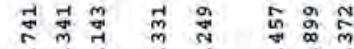

$3 d$

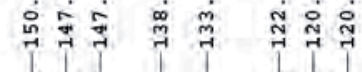

$\mid$

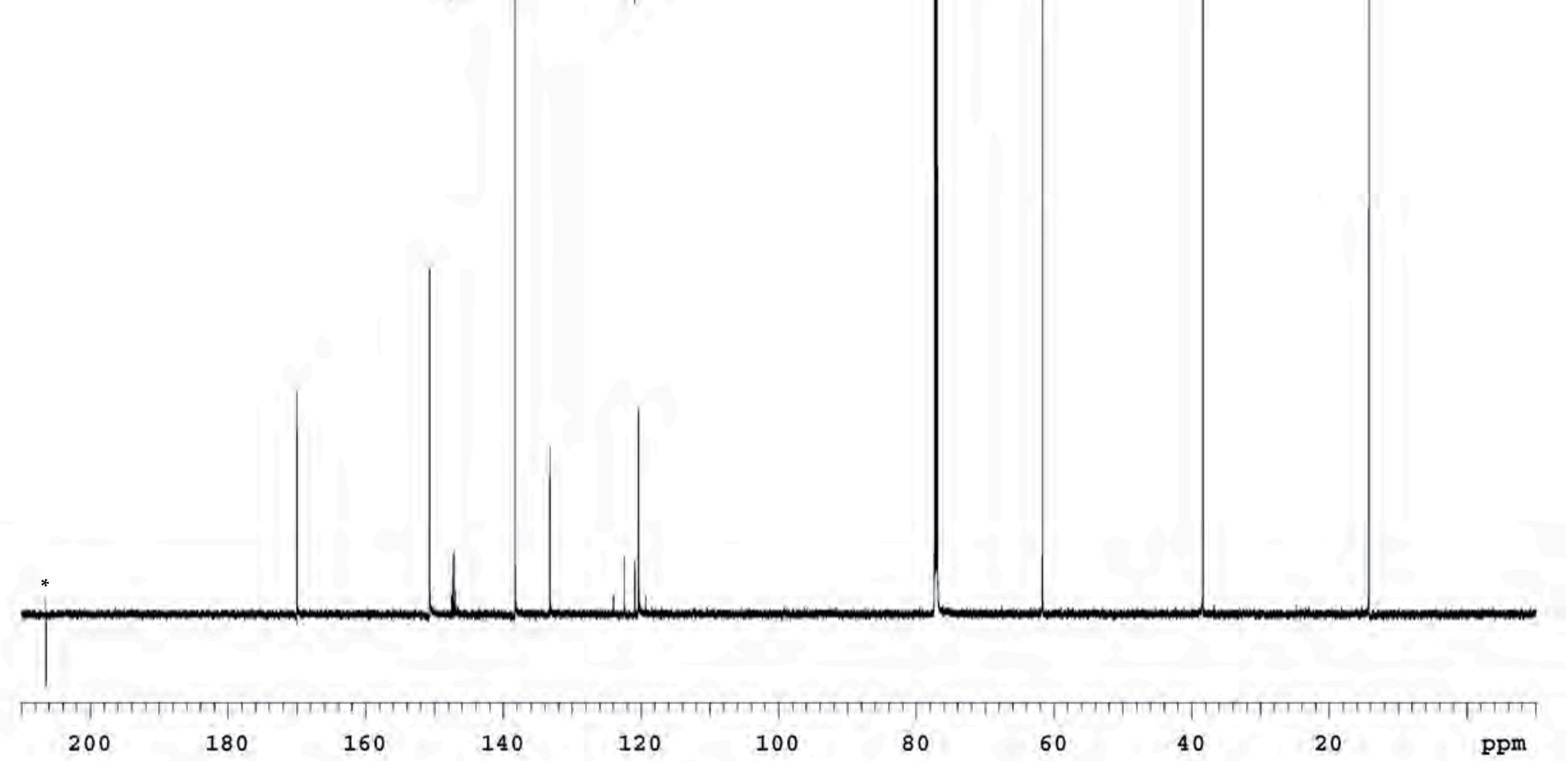


Department of Chemistry, University of Alberta

Agilent Technologies

Recopdedoit v700, Mar 72016

Sweop Wellaikar $\quad \mathbf{8 3 8 9 . 2 6}$

Pulse Sequenoe PRESAT

Drgital Restiraip) $\quad \mathbf{0 . 1 3}$

Acouralian Time(g);

Ha par min(Hz/mmi): $\quad 32.07$

Tilaxalund Colaysy 0.1

Shengkang, SY-03-93

699.762 MHz H1 PRESAT in cdcl3 (ret. to CDCl3 @ $7.26 \mathrm{ppm}$ ), temp $27.5 \mathrm{C} \rightarrow$ actual temp $=27.0 \mathrm{C}$, coldid probe<smiles>O=C(Cc1cccnc1F)OBr</smiles>

$3 e$

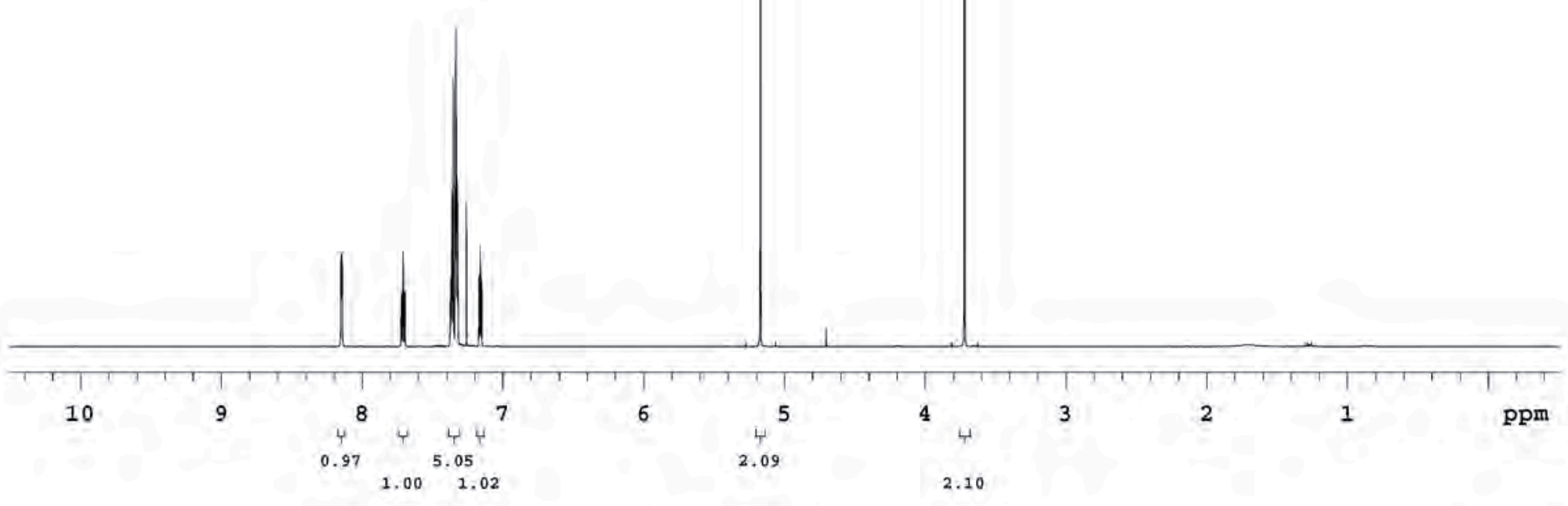

S-79 


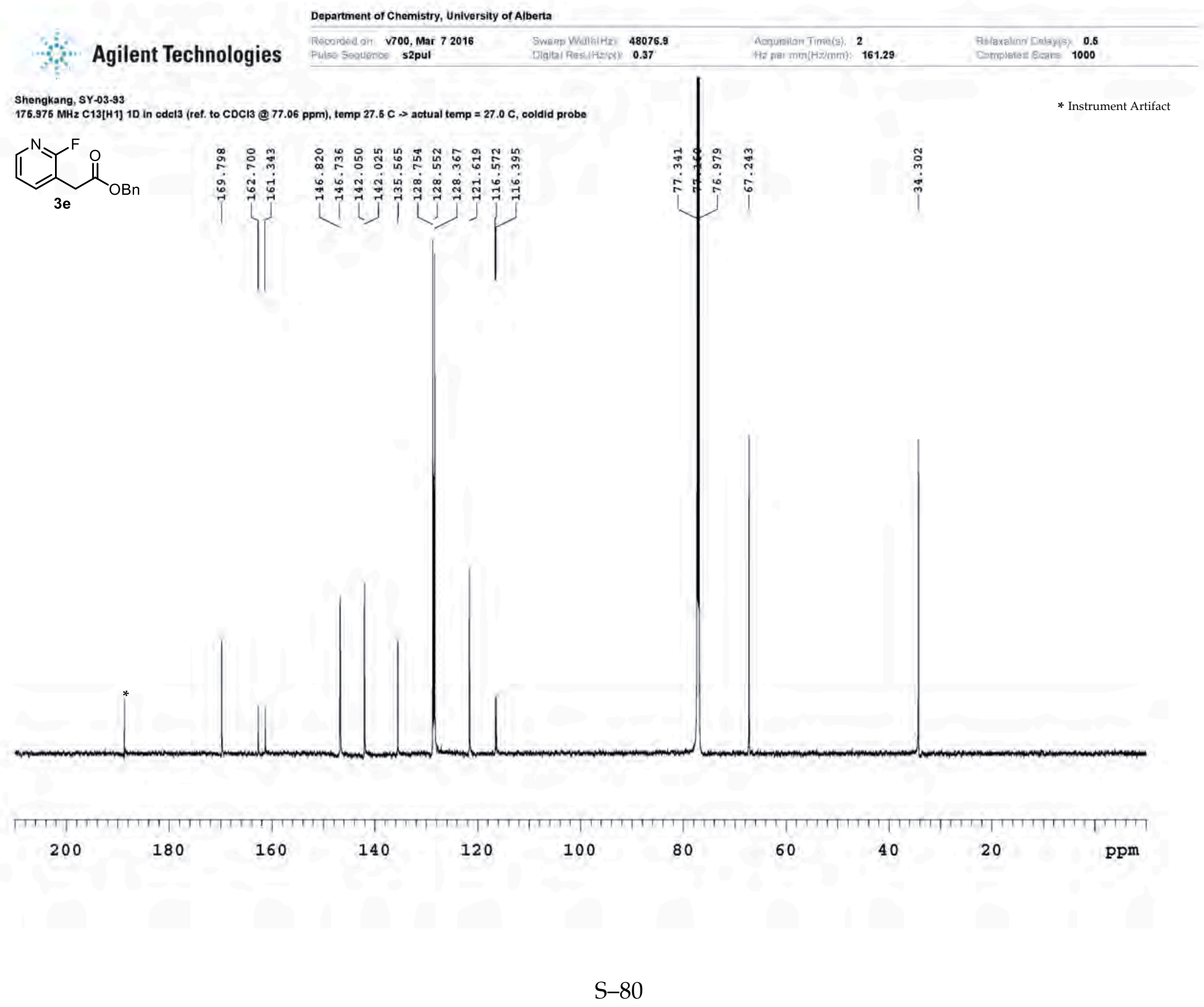


Department of Chemistry, University of Alberta

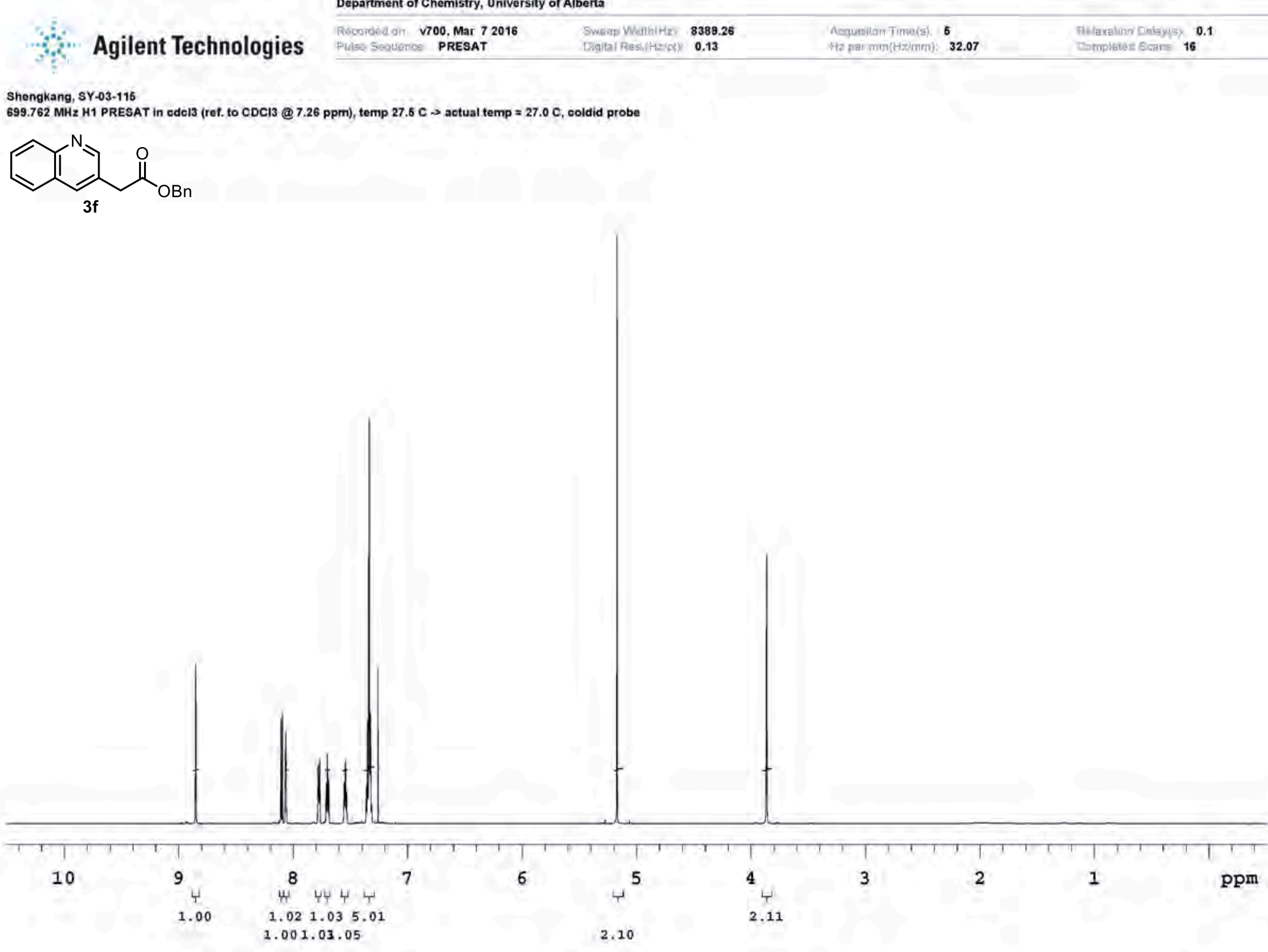




\section{Department of Chemistry, University of Alberta}

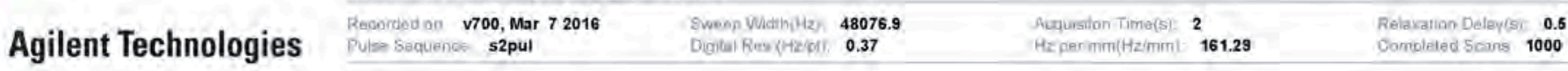
Shengkang, SY-03-115 $175.975 \mathrm{MHz} C 13[\mathrm{H} 1] 1 \mathrm{D}$ in cdel3 (ref. to $\mathrm{CDC} 13$ \& $77.06 \mathrm{ppm}$ ), temp $27.6 \mathrm{C} \rightarrow$ actual temp $=27.0 \mathrm{C}$, coldid probe

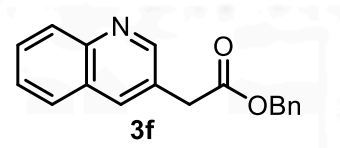

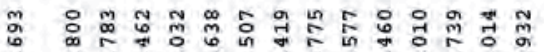

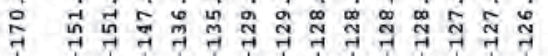

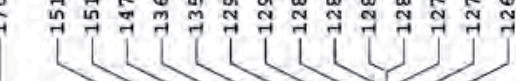

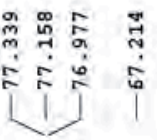

* Instrument Artifact

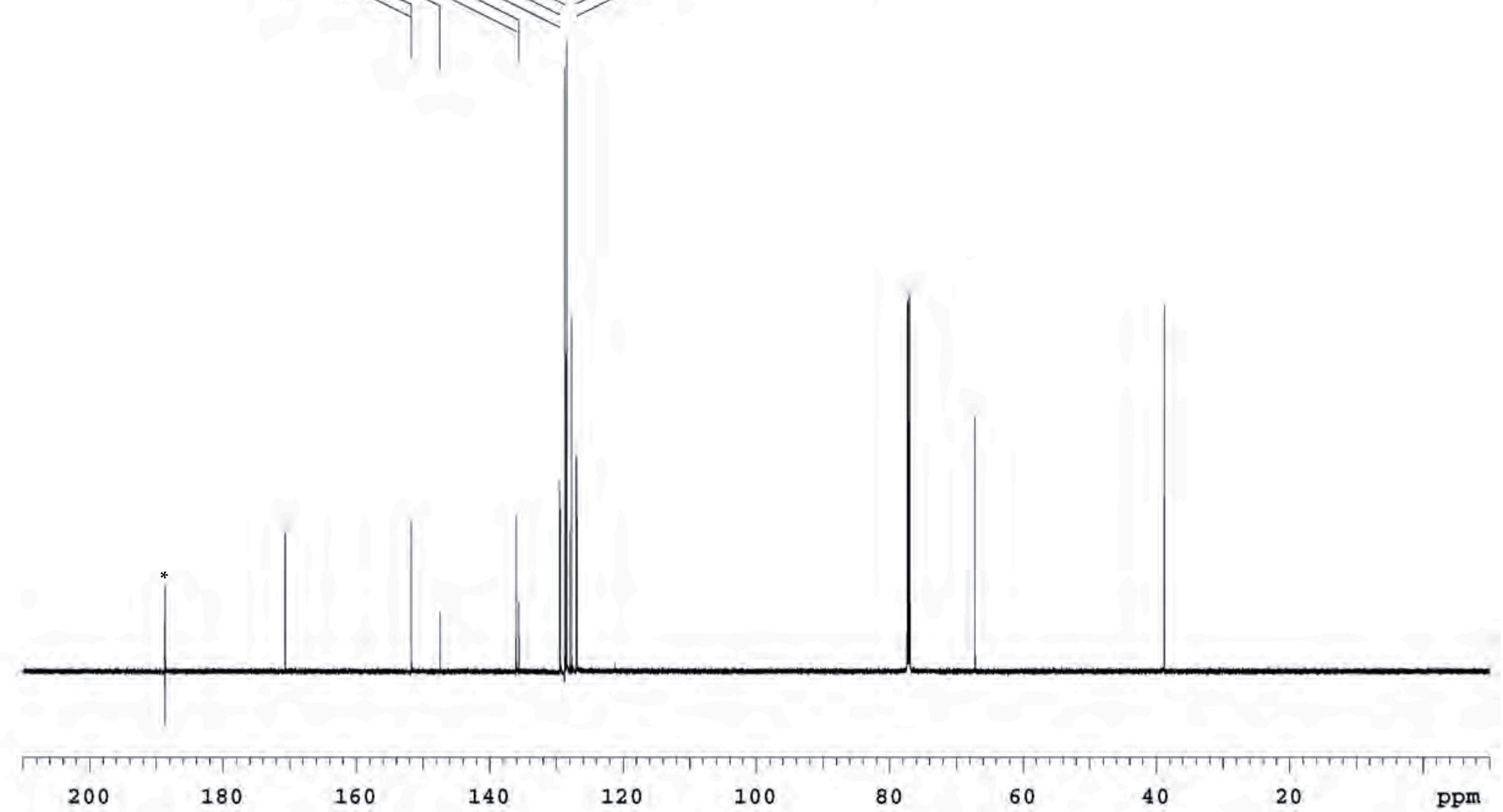


Department of Chemistry, University of Alberta
Agilent Technologies
Recordela oir v700, Apr 132016

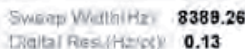

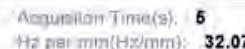 Tibaxalion Delayysy 0.1

Shengkang, SY-03-173-C3-H

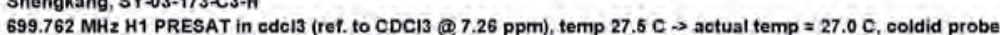
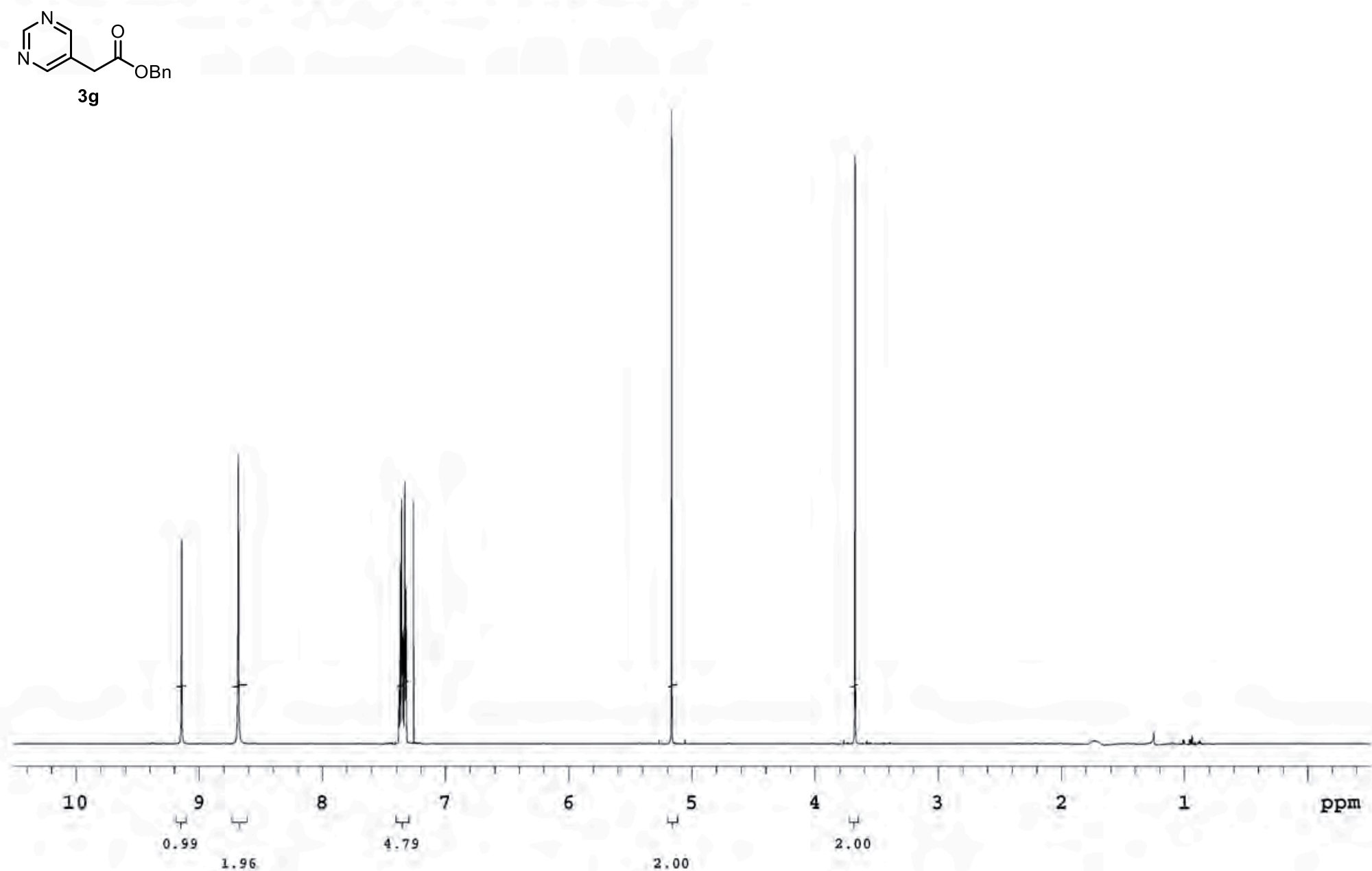


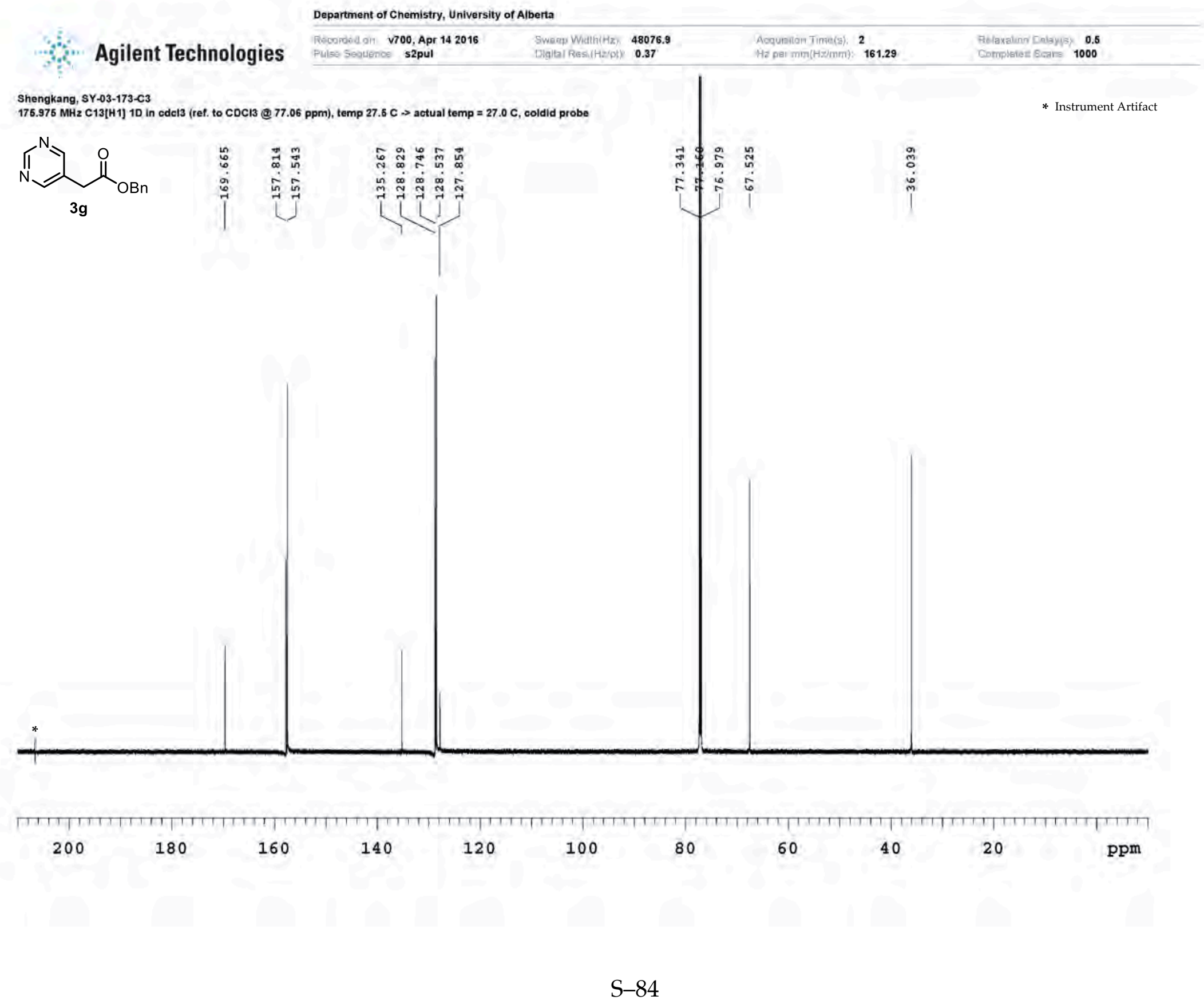


Department of Chemistry, University of Alberta

Agilent Technologies Pulso Seatencos PRESAT

Shengkang, SY-03-139-1

(99.)<smiles>COc1ncc(CC(=O)Br)cn1</smiles>

3h

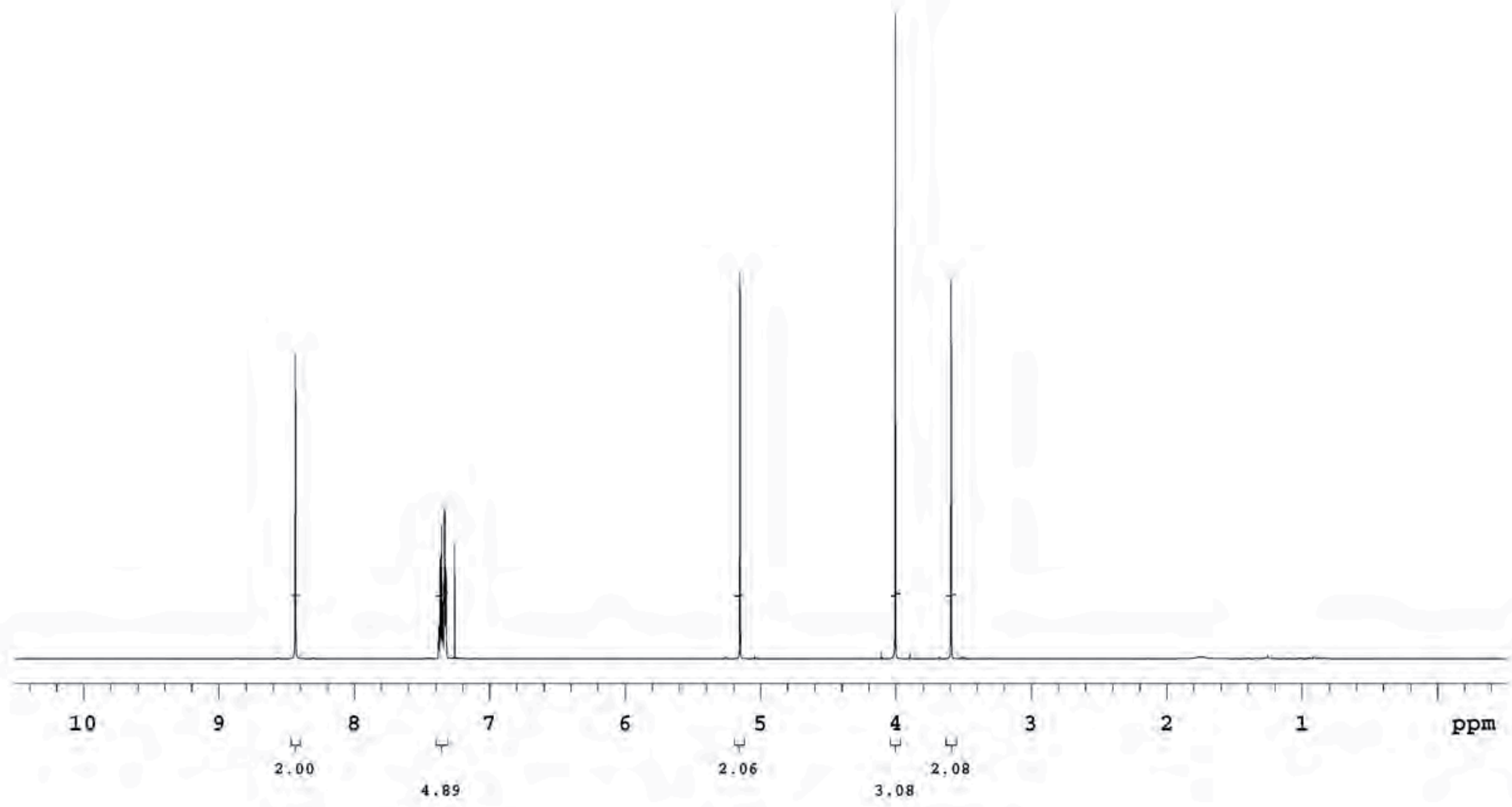




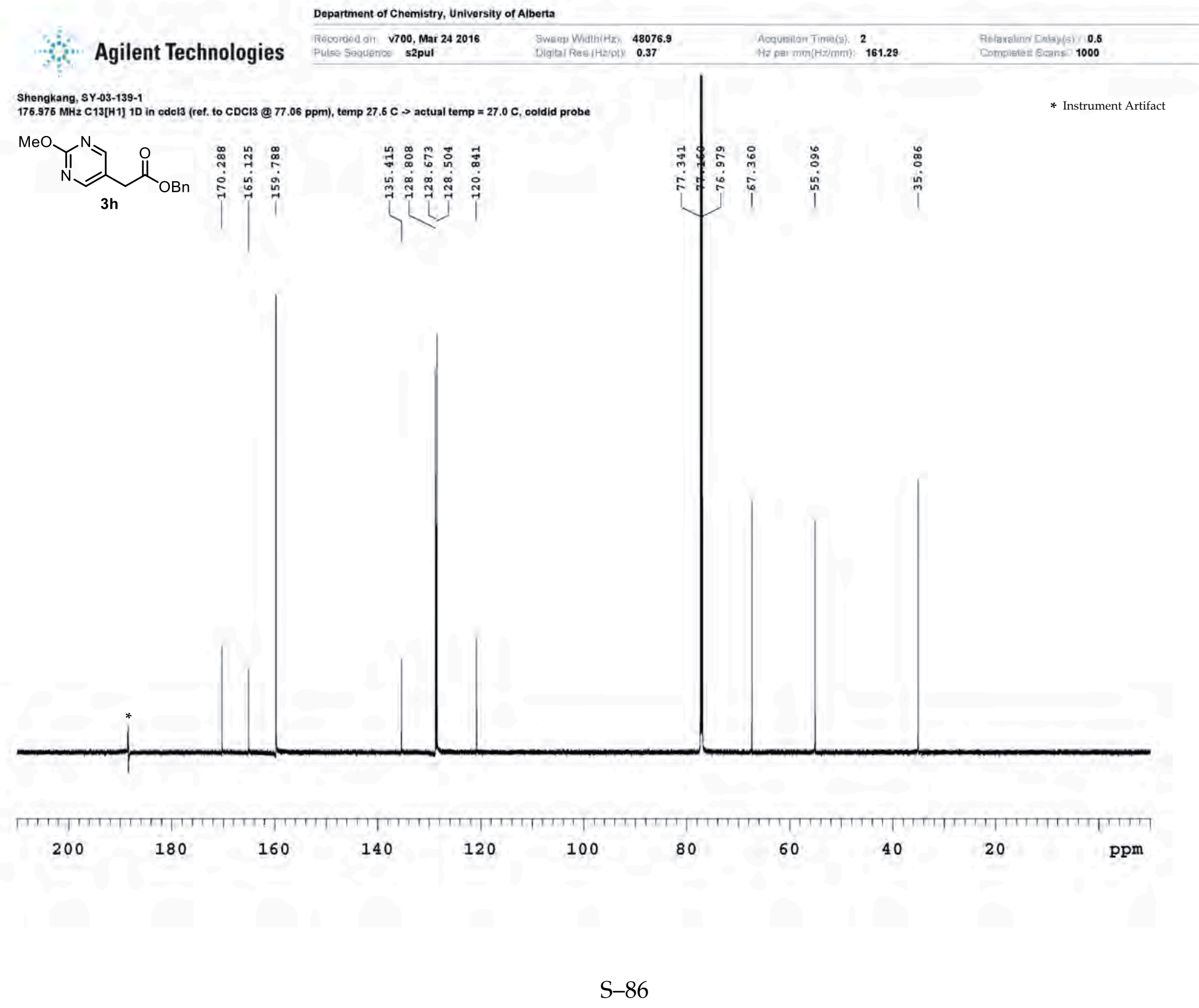


Department of Chemistry, University of Alberta
Agilent Technologies
Recorded on v700, Mar 72016

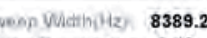
8389.26
0.13
Auguastion Timie(s): ${ }^{6}$
Relaxatian Deley(s) 0.1

Shengkang, SY-03-107-1

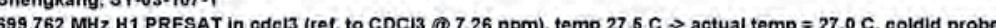

${ }_{\mathrm{N}}^{\mathrm{Cl}}$

$3 \mathbf{i}$

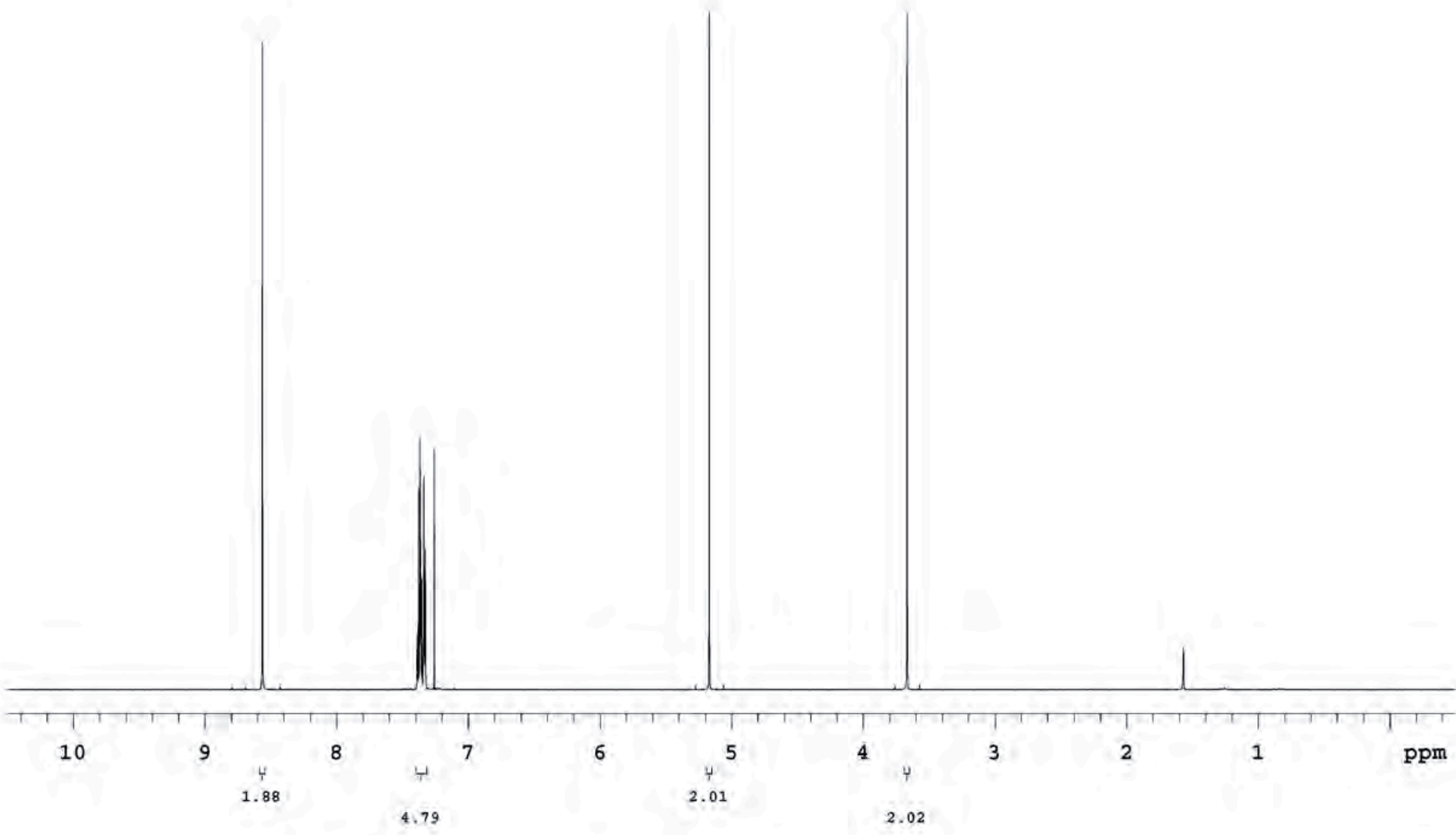




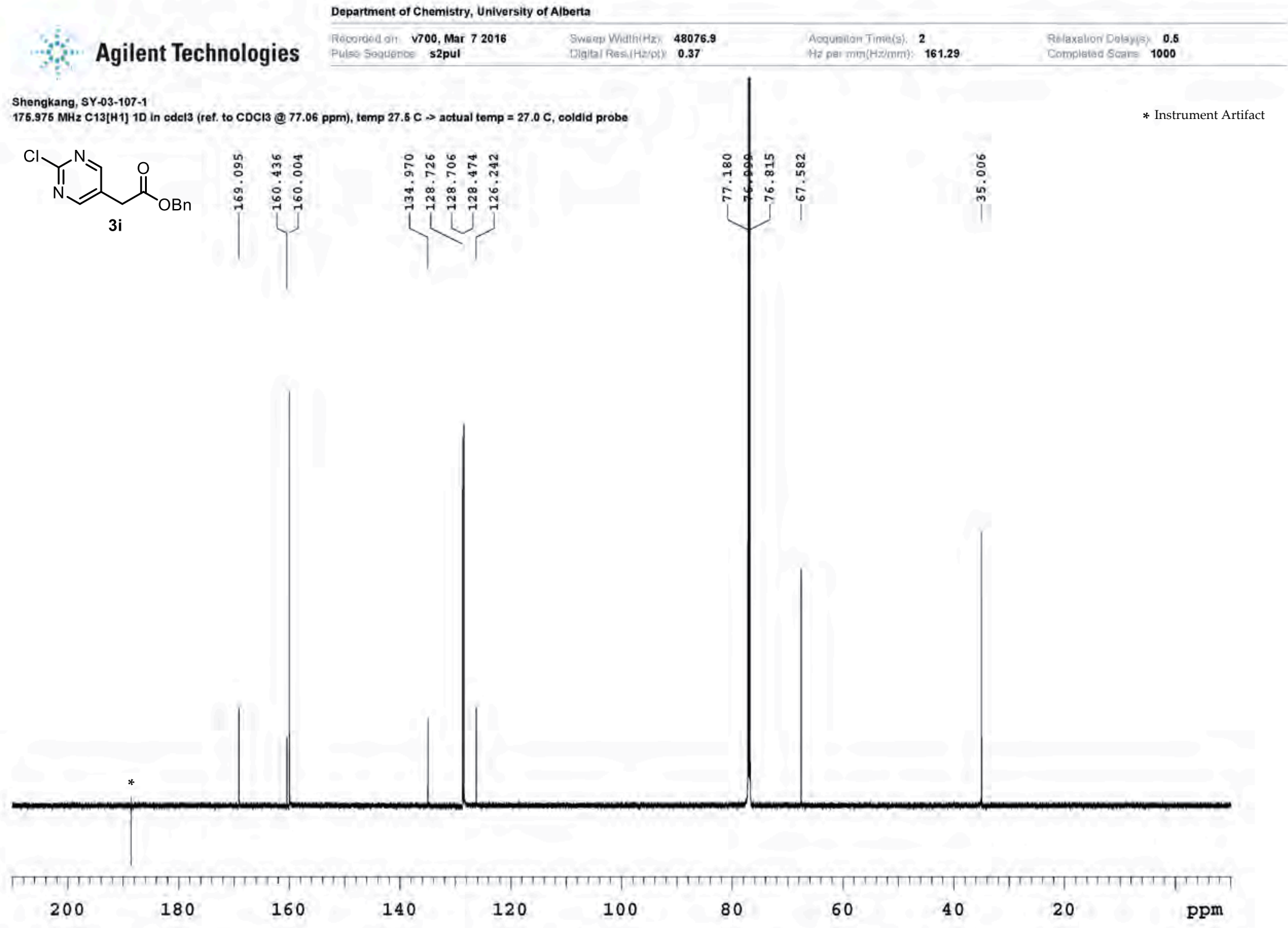


Department of Chemistry, University of Alberta

\begin{tabular}{|c|c|c|c|c|}
\hline Aailent Technoloaies & $\begin{array}{l}\text { Recorded on: u500, Feb } 32016 \\
\text { Pulse Sequence: PRESAT }\end{array}$ & 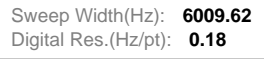 & 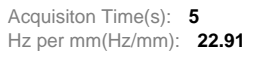 & $\begin{array}{l}\text { Relaxation Delay(s): } \mathbf{0 . 1} \\
\text { Completed Scans } \mathbf{1 6}\end{array}$ \\
\hline
\end{tabular}

Patrick, PM-06-173-A-F23-26

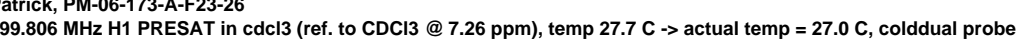

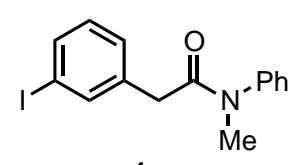

$4 a$

Me

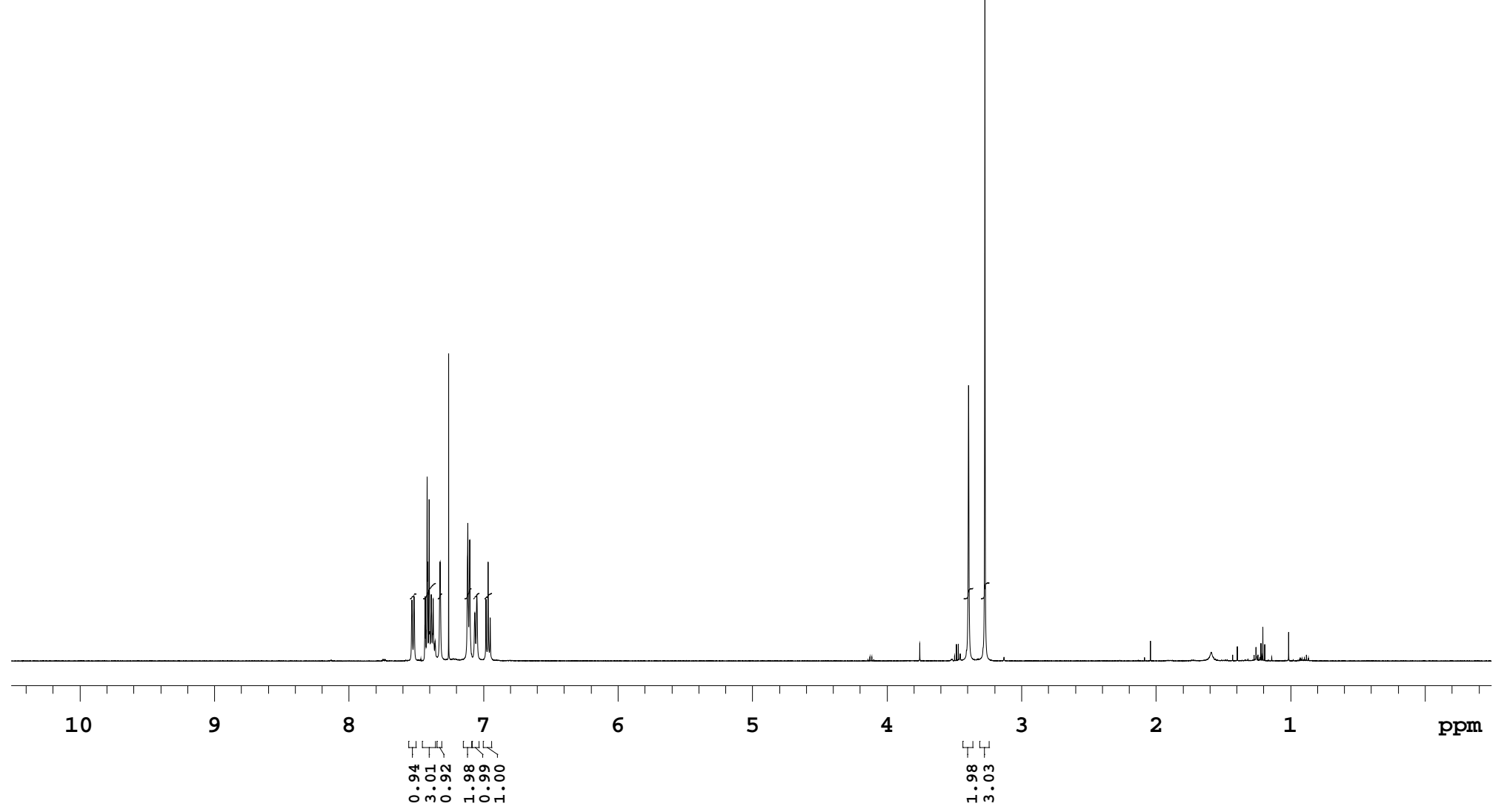

File: /mnt/d600/home13/rilnmr/hmmrdata/DATA_FROM_NMRSERVICE/Patrick/2016.02/2016.02.03.u5_PM-06-173-A-F23-26_loc1_03.00_H1_1D 
Department of Chemistry, University of Alberta

\begin{tabular}{|c|c|c|c|}
\hline Arilent Tochnolnaips & $\begin{array}{l}\text { Recorded on: u500, Feb } \mathbf{3} 2016 \\
\text { Pulse Sequence: s2pul }\end{array}$ & $\begin{array}{l}\text { Acquisiton Time(s): } 2.5 \\
\text { Hz per } m m(H z / m): 115\end{array}$ & $\begin{array}{l}\text { Relaxation Delay(s): } \mathbf{0 .} \\
\text { Completed S Sans } \mathbf{2 5 6}\end{array}$ \\
\hline
\end{tabular}

Patrick, PM-06-173-A-F23-26

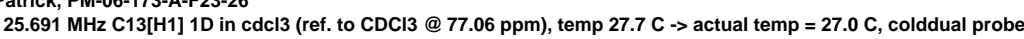

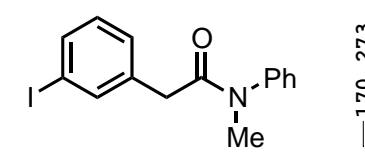

กิ

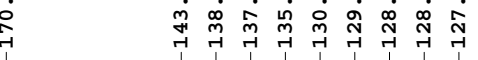

$4 a$

$\stackrel{4}{\frac{2}{2}}$

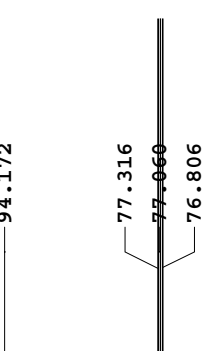

$\stackrel{\circ}{\circ}$

桨

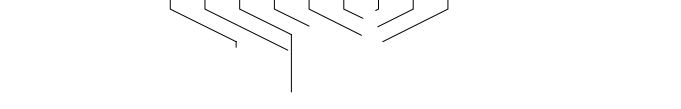




\section{Department of Chemistry, University of Albert}

\begin{tabular}{|c|c|c|c|c|}
\hline Aailent Technolonie & $\begin{array}{l}\text { Recorded on: ibd5, Sep } 212016 \\
\text { Pulse Sequence: s2pul }\end{array}$ & $\begin{array}{l}\text { Sweep Width(Hz): } \quad \mathbf{6 0 0 0 . 6} \\
\text { Digital Res.(Hz/pt): } \mathbf{0 . 0 9}\end{array}$ & $\begin{array}{l}\text { Acquisiton Time(s): } \mathbf{5} \\
\text { Hz per } \mathrm{mm}(\mathrm{Hz} / \mathrm{mm}): \mathbf{2 2 . 8 2}\end{array}$ & $\begin{array}{l}\text { Relaxation Delay(s): } \mathbf{0 . 1} \\
\text { Completed Scans } \mathbf{4 4}\end{array}$ \\
\hline
\end{tabular}

PM-06-175-10

498.118 MHz H1 1D in cdcl3 (ref. to CDCl3 @ 7.26 ppm), temp 26.4 C > actual temp = 27.0 C, autoxdb probe

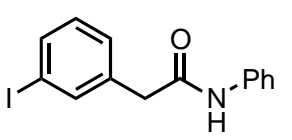

$4 b$

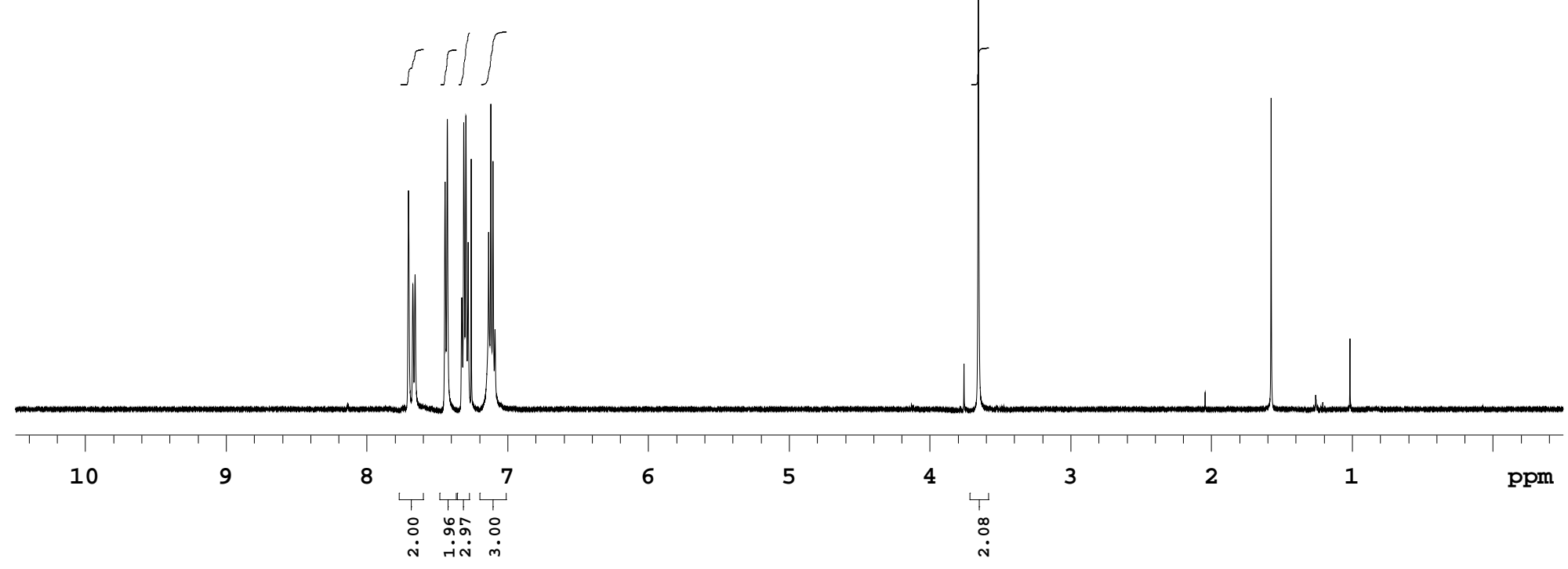


Department of Chemistry, University of Alberta

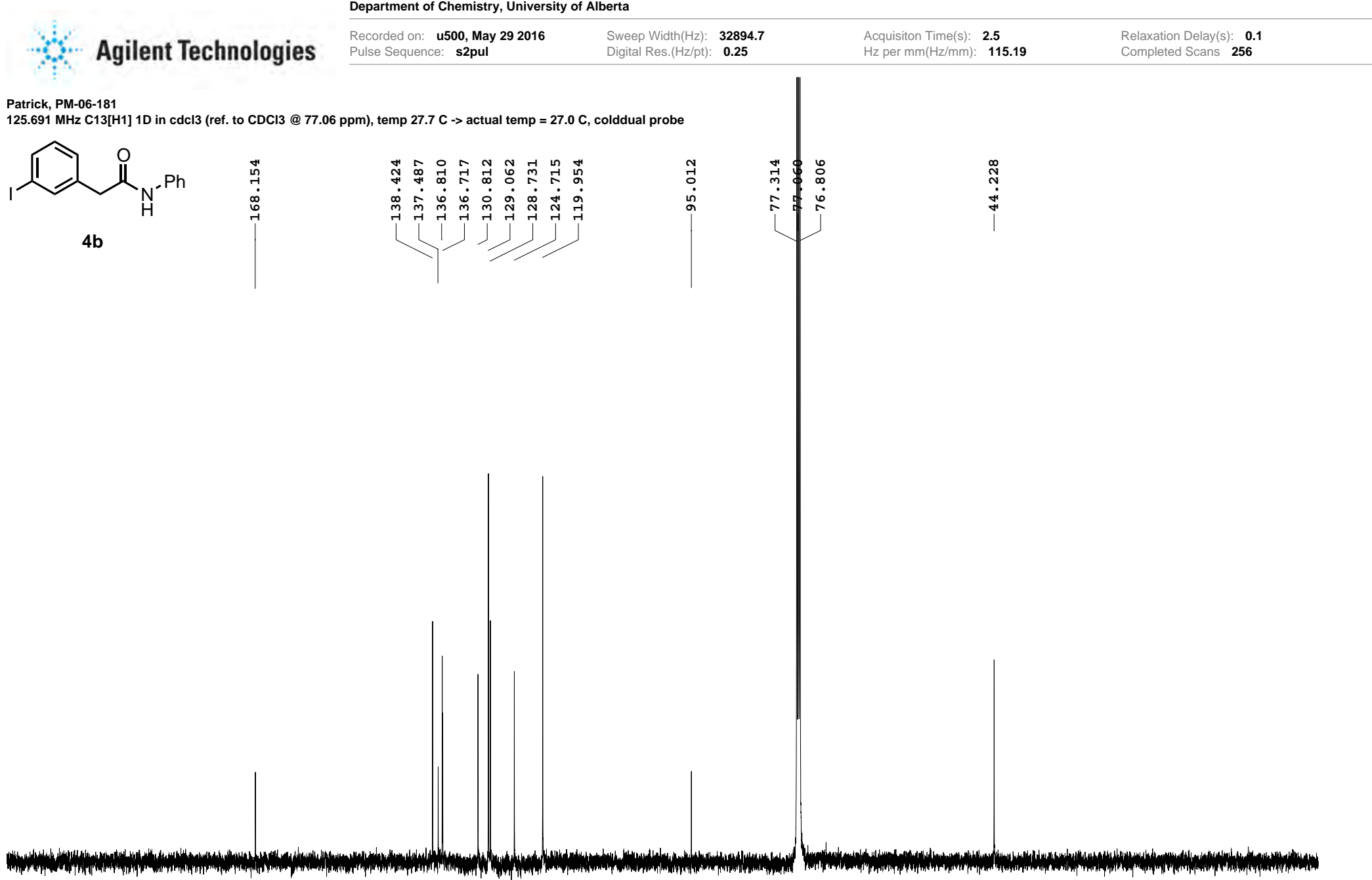

\begin{tabular}{|c|c|c|c|c|}
\hline 200 & 180 & 160 & 140 & 120 \\
\hline
\end{tabular}


Department of Chemistry, University of Alberta

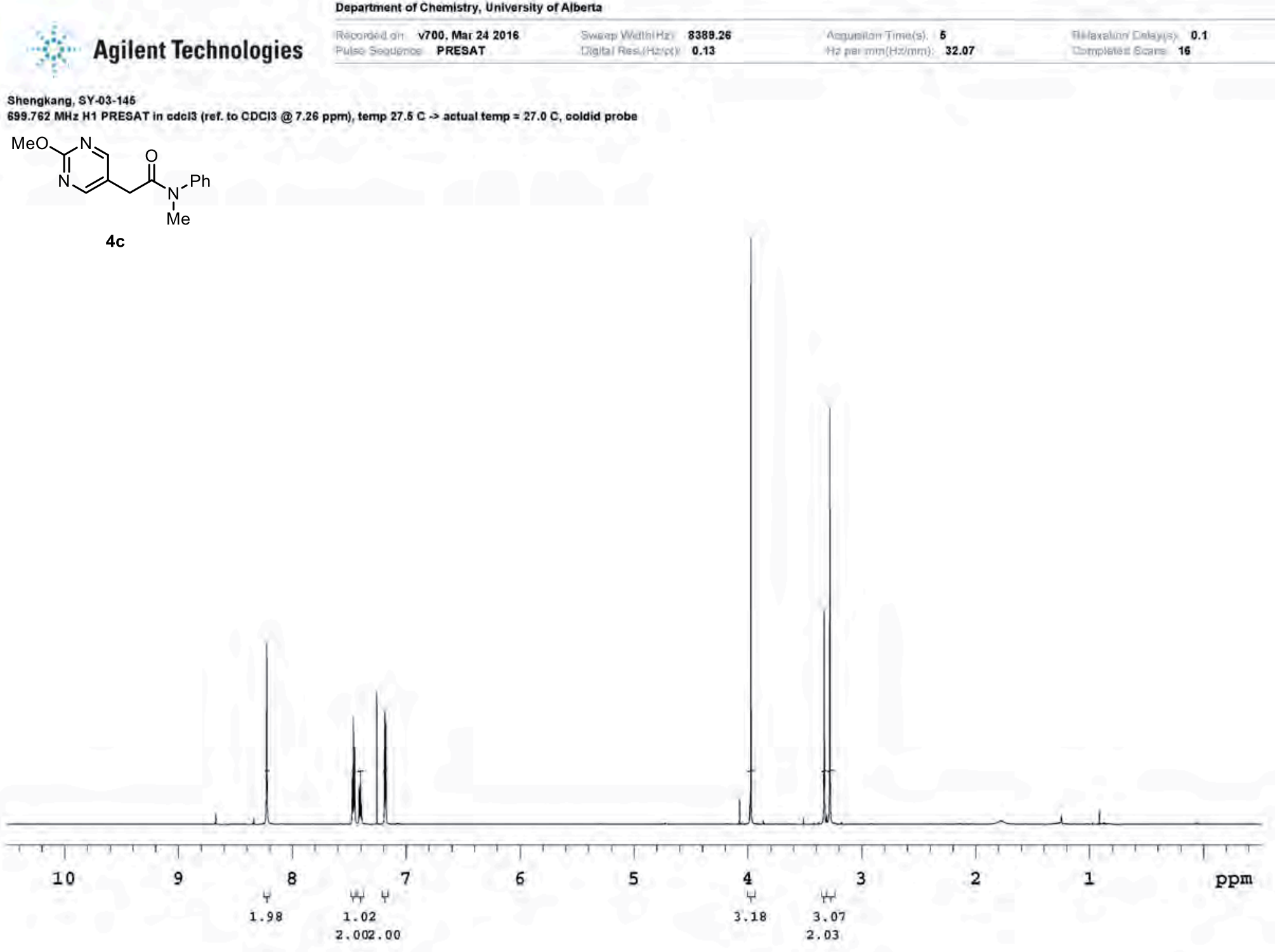


Department of Chemistry, University of Albert

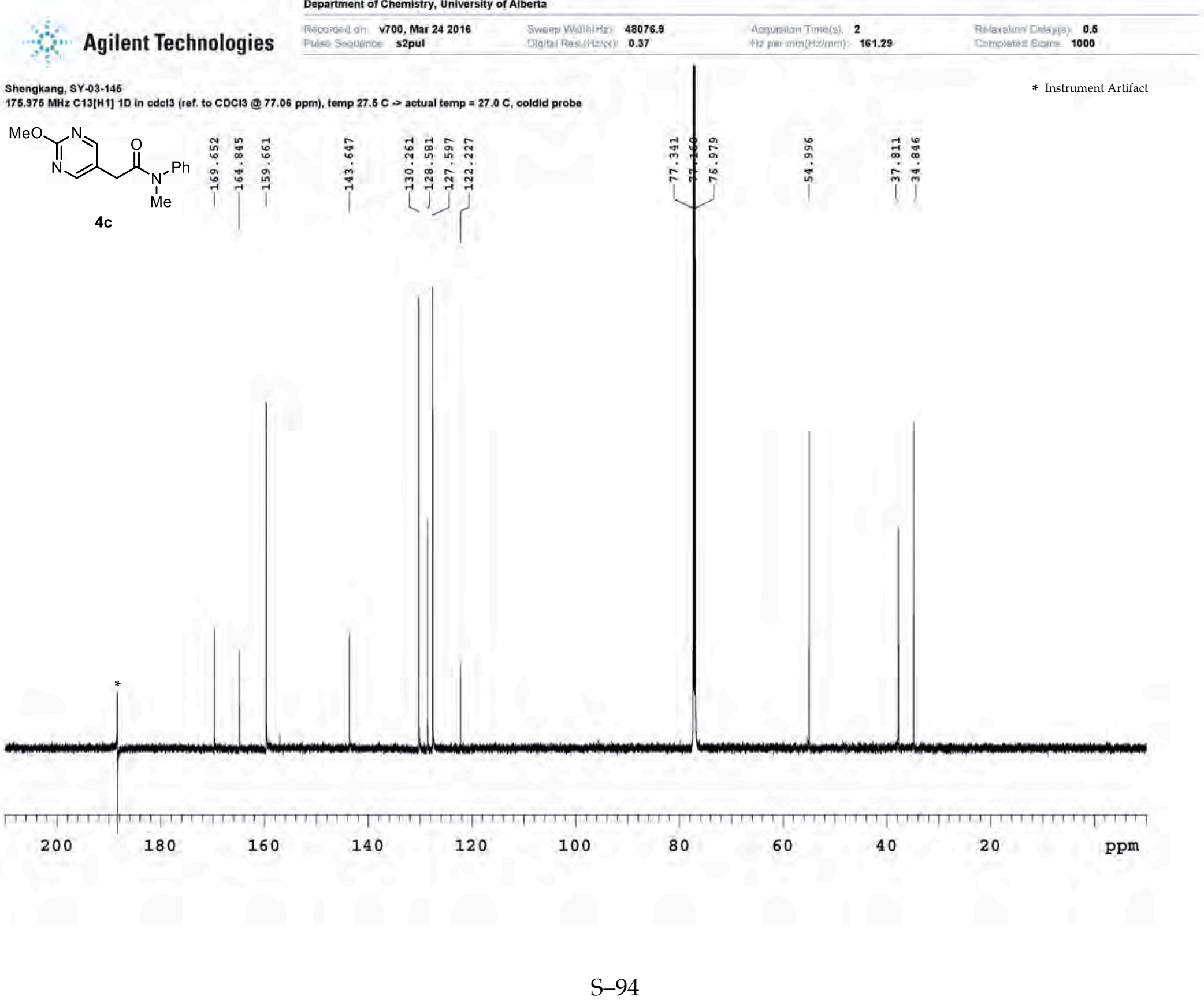


Agilent Technologies

Recorded on: mr400, Dec 22015

Pulse Sequence: s2pul
Sweep Width $(\mathrm{Hz}): \mathbf{4 8 0 7 . 6 9}$

Digital Res.(Hz/pt): $\mathbf{0 . 0 7}$
Acquisition Time (s): $\mathbf{5}$

$\mathrm{Hz}$ per $\mathrm{mm}(\mathrm{Hz} / \mathrm{mm}): \mathbf{1 8 . 3 5}$
Relaxation Delay (s): $\mathbf{0 . 1}$

Completed Scans $\mathbf{4 0}$

PM-06-051-4

399.984 MHz H1 1D in cdcl3 (ref. to CDCl3 @ 7.26 ppm), temp 25.9 C -> actual temp = 27.0 C, onenmr probe<smiles>O=C(Cc1cccc(Br)c1)N1CCCC1</smiles>

id

$\int \zeta$
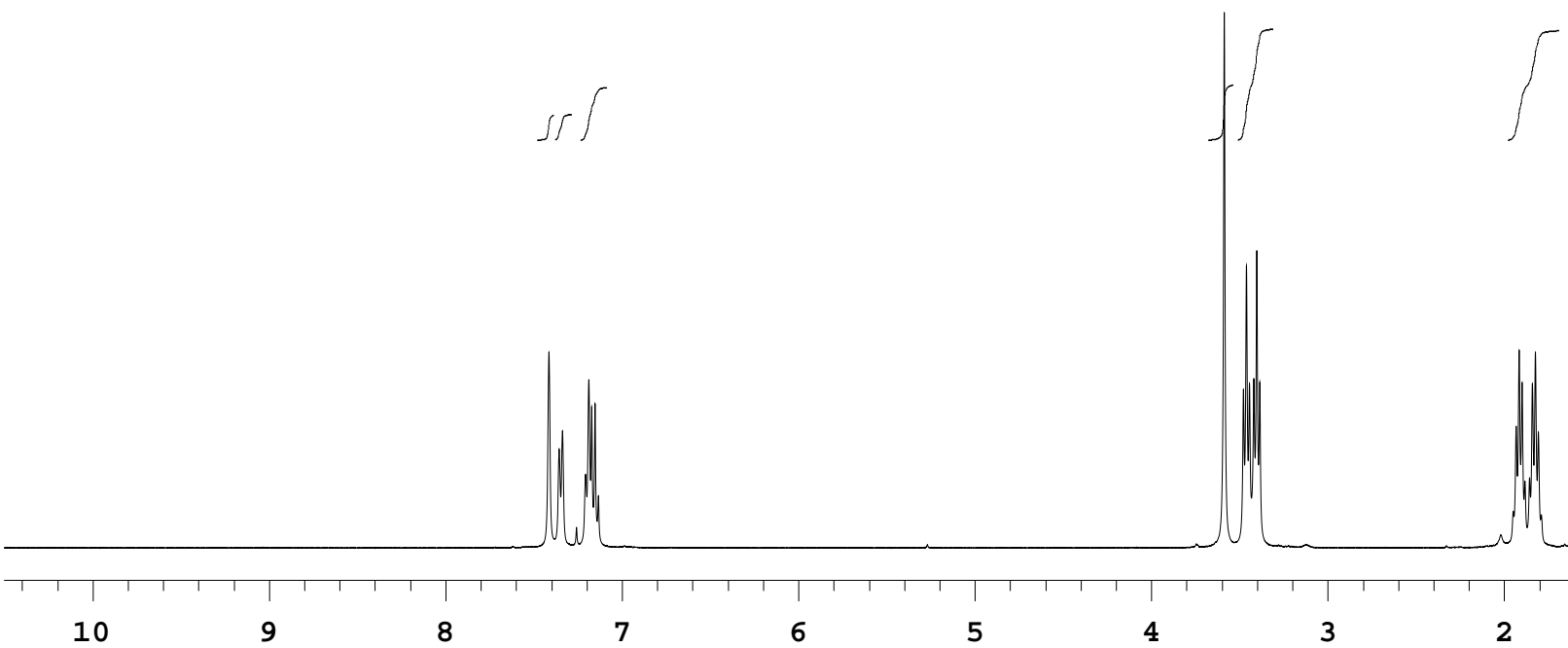

8

7

6

5

4

3

soำ

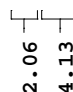

2

1

ppm

File: /mnt/d600/home13/rjlnmr/nmrdata/Patrick/2015.12/2015.12.02.mr4_PM-06-051-4_H1_1D

S-95 
Department of Chemistry, University of Alberta

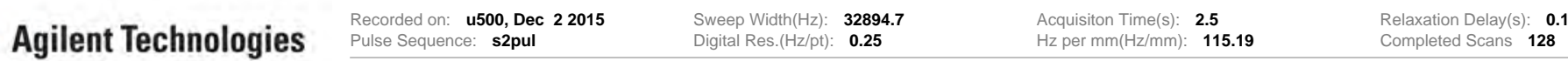

Patrick, PM-06-051-4

125.691 MHz C13[H1] 1D in cdcl3 (ref. to CDCl3 @ 77.06 ppm), temp $27.7 \mathrm{C}$-> actual temp = 27.0 C, colddual probe

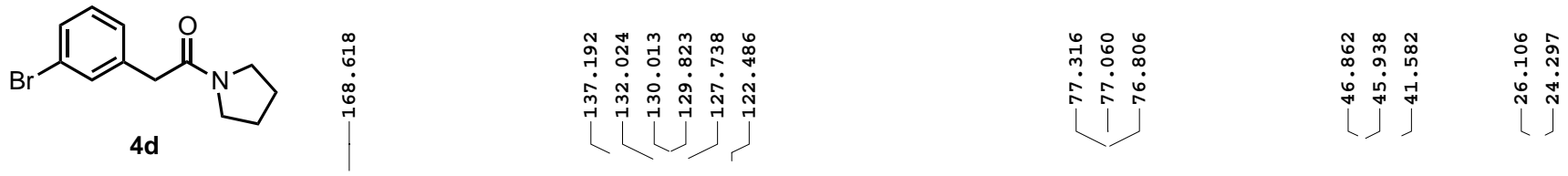

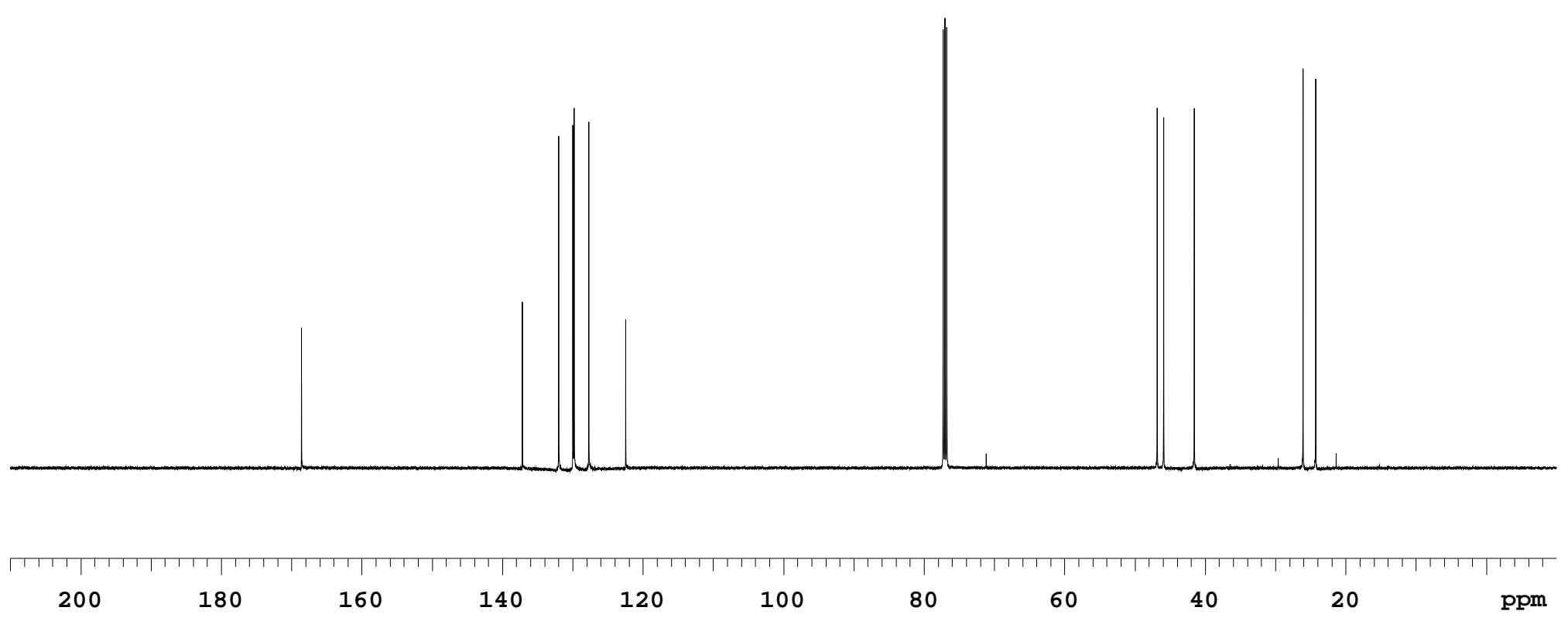

File: /mnt/d600/home13/rijnmr/nmrdata/DATA_FROM_NMRSERVICE/Patrick/2015.12/2015.12.02.u5_PM-06-051-4_loc8_14.20_C13_1D 
Department of Chemistry, University of Alberta

\begin{tabular}{|c|c|c|c|c|}
\hline Aailent Technoloaies & $\begin{array}{l}\text { Recorded on: u500, Feb } 52016 \\
\text { Pulse Sequence: PRESAT }\end{array}$ & 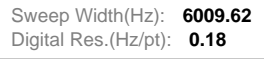 & $\begin{array}{l}\text { Acquisiton Time }(\mathrm{s}): \mathbf{5} \\
\mathrm{Hz} \text { per } \mathrm{mm}(\mathrm{Hz} / \mathrm{mm}): \mathbf{2 2 . 9}\end{array}$ & $\begin{array}{l}\text { Relaxation Delay(s): } \mathbf{0 . 1} \\
\text { Completed Scans } \mathbf{1 6}\end{array}$ \\
\hline
\end{tabular}

Patrick, PM-06-169-F10-15

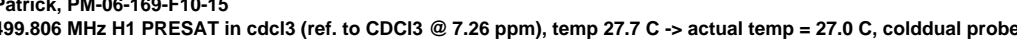

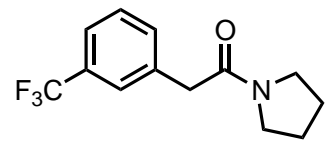

$4 e$

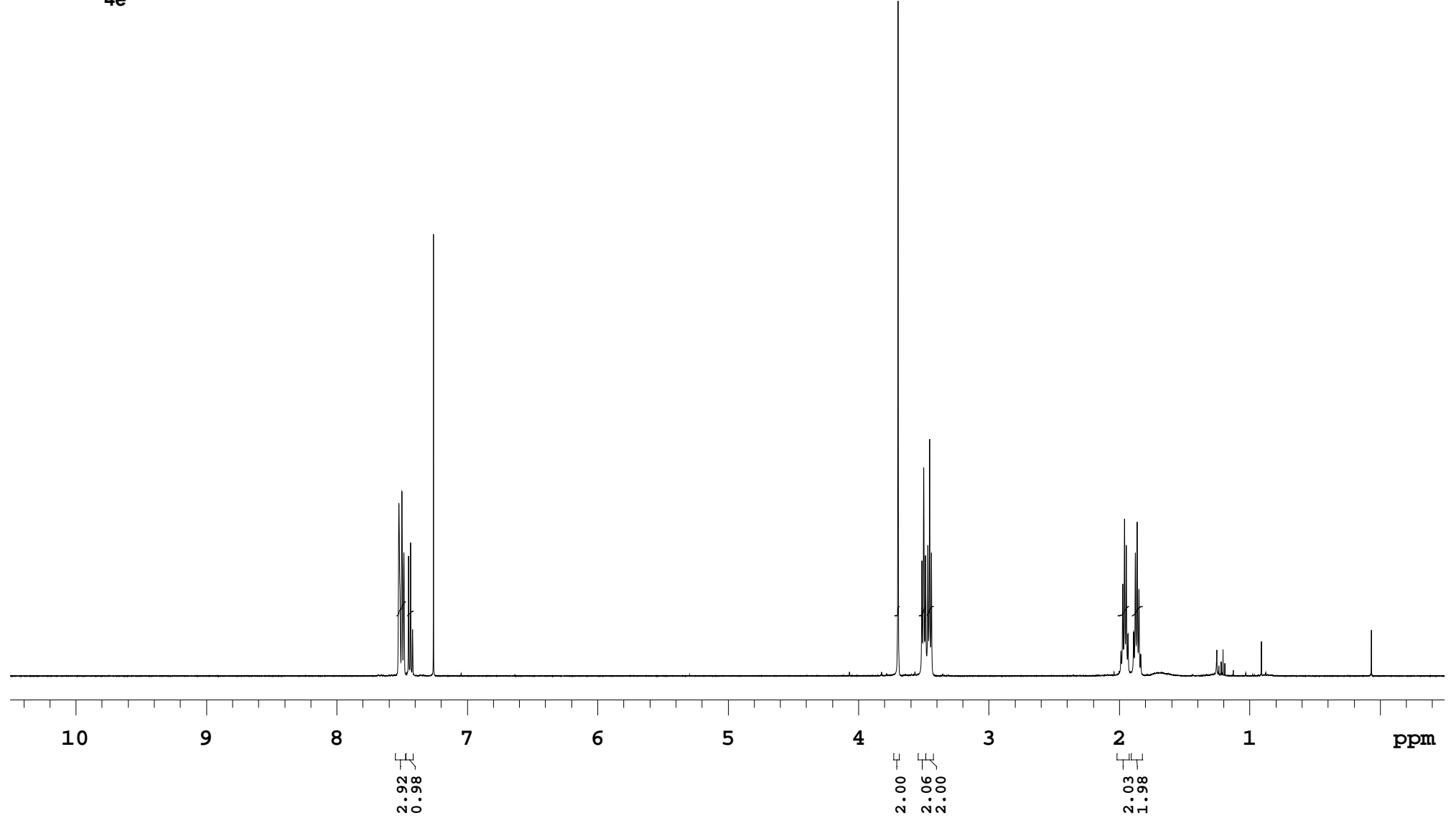

File: /mnt/d600/home13/rilnmr/hmrdata/DATA_FROM_NMRSERVICE/Patrick/2016.02/2016.02.05.u5_PM-06-169-F10-15_loc12_14.31_H1_1D 
Department of Chemistry, University of Alberta

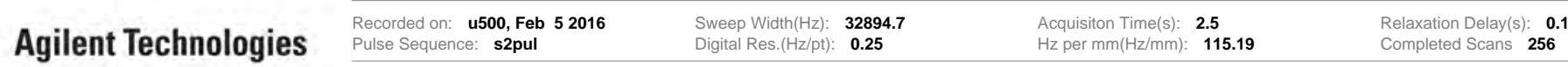

Patrick, PM-06-169-F10-15

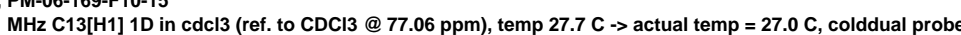

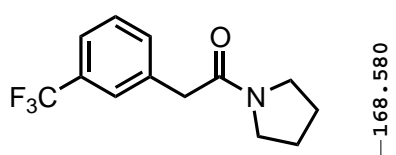

$4 e$

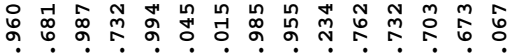

$\dot{\vec{m}}$

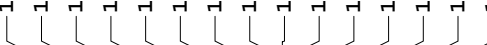

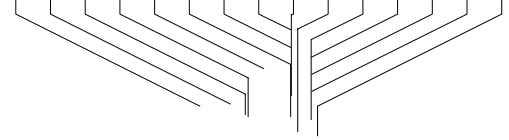

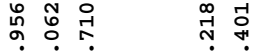

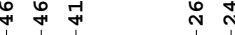

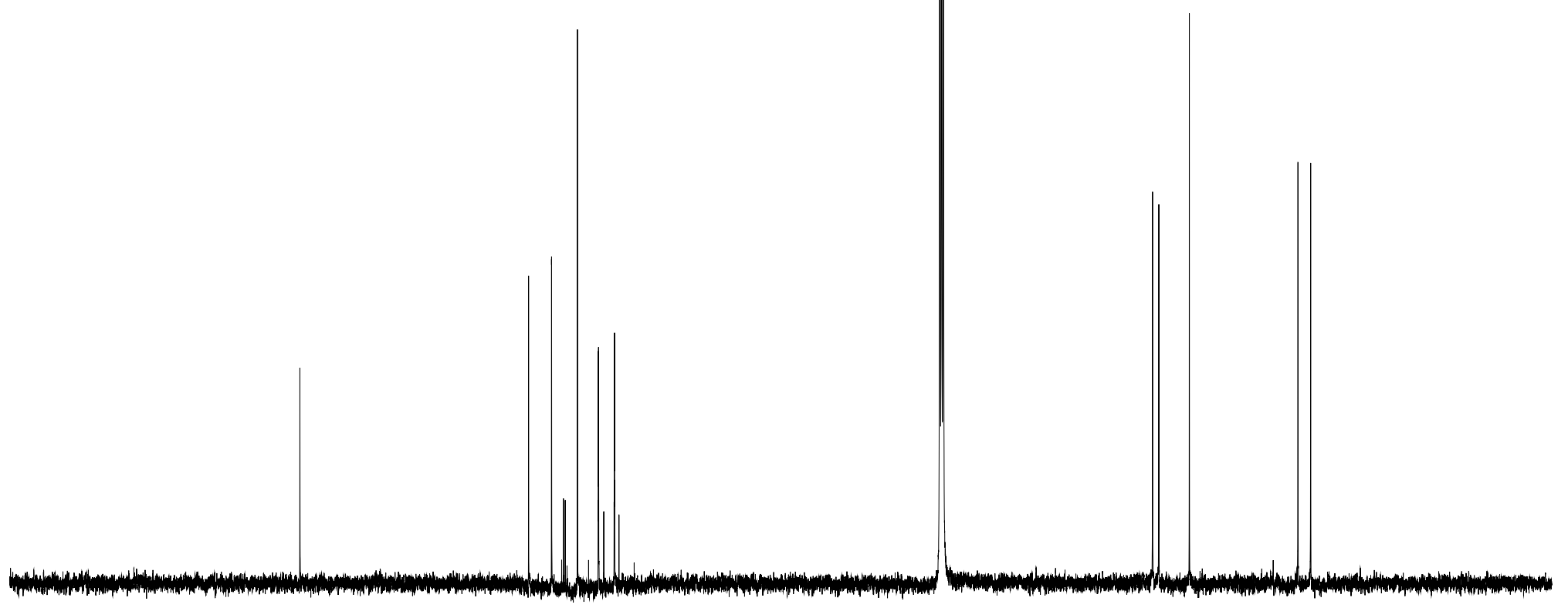

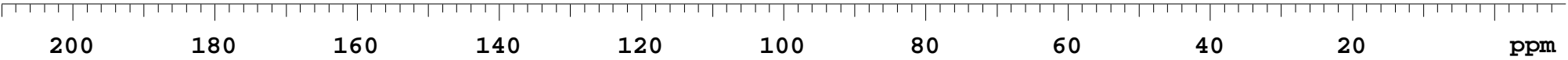


Department of Chemistry, University of Alberta

\begin{tabular}{|c|c|c|c|c|c|}
\hline Anilent Technolon & $\begin{array}{l}\text { Recorded on: mr400, Dec } 22015 \\
\text { Pulse Sequence: s2pul }\end{array}$ & $\begin{array}{l}\text { Sweep Width( }(H z): 4 \\
\text { Digital Res. }(H z / p t):\end{array}$ & $\begin{array}{l}4807.69 \\
0.07\end{array}$ & $\begin{array}{l}\text { Acquisiton Time(s): } \mathbf{5} \\
\text { Hz per } \mathrm{mm}(\mathrm{Hz} / \mathrm{mm}): \mathbf{1 8 . 3 2}\end{array}$ & $\begin{array}{l}\text { Relaxation Delay(s): } 0.1 \\
\text { Completed Scans } \mathbf{2 8}\end{array}$ \\
\hline
\end{tabular}

PM-06-041-4

399.984 MHz H1 1D in cdcl3 (ref. to CDCl3 @ 7.26 ppm), temp 25.9 C -> actual temp = 27.0 C, onenmr probe

$\overbrace{\substack{\mathrm{N}^{-} \\ \mathrm{Me}}}^{\mathrm{O}}$

$4 f$

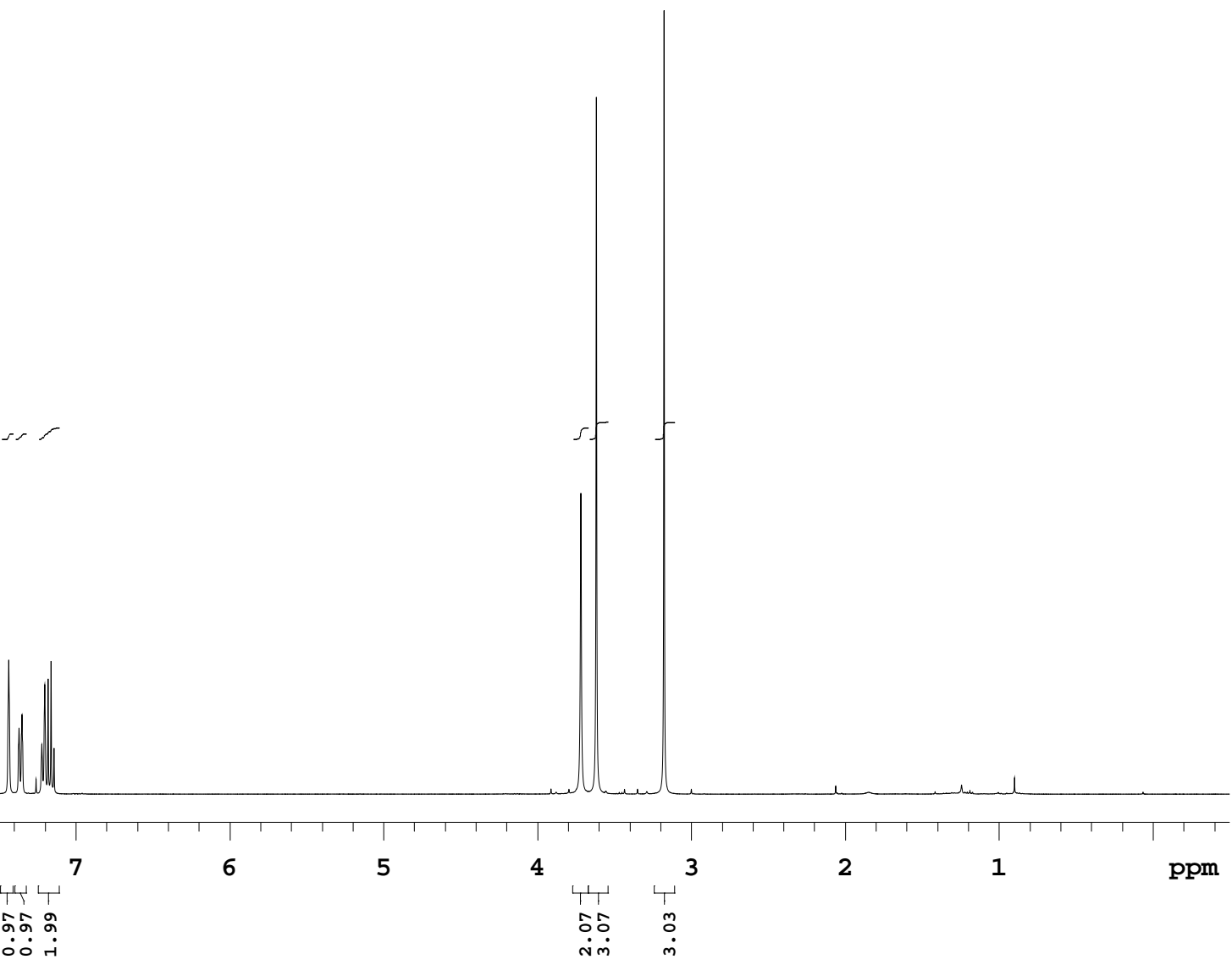

File: /mnt/d600/home13/rilnmr/nmrdata/Patrick/2015.12/2015.12.02.mr4_PM-06-041-4_H1_1D 
Department of Chemistry, University of Alberta

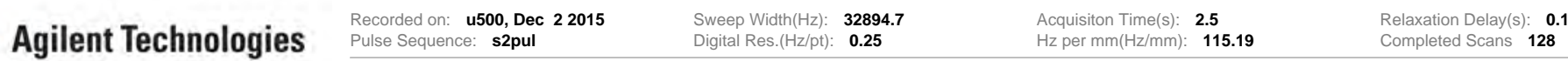

Patrick, PM-06-041-4

$125.691 \mathrm{MHz}$ C13[H1] $1 \mathrm{D}$ in cdcl3 (ref. to CDCl3 @ $77.06 \mathrm{ppm}$ ), temp $27.7 \mathrm{C}>$ actual temp = 27.0 C, colddual probe

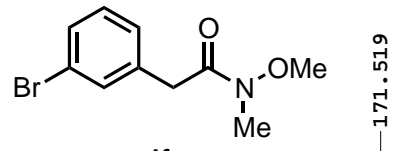

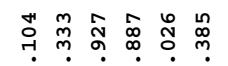

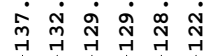

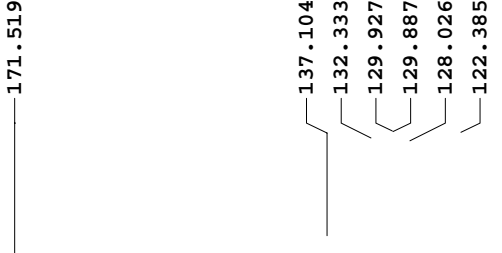

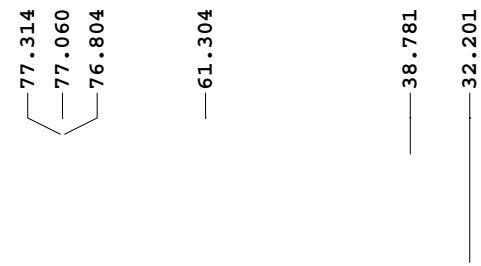

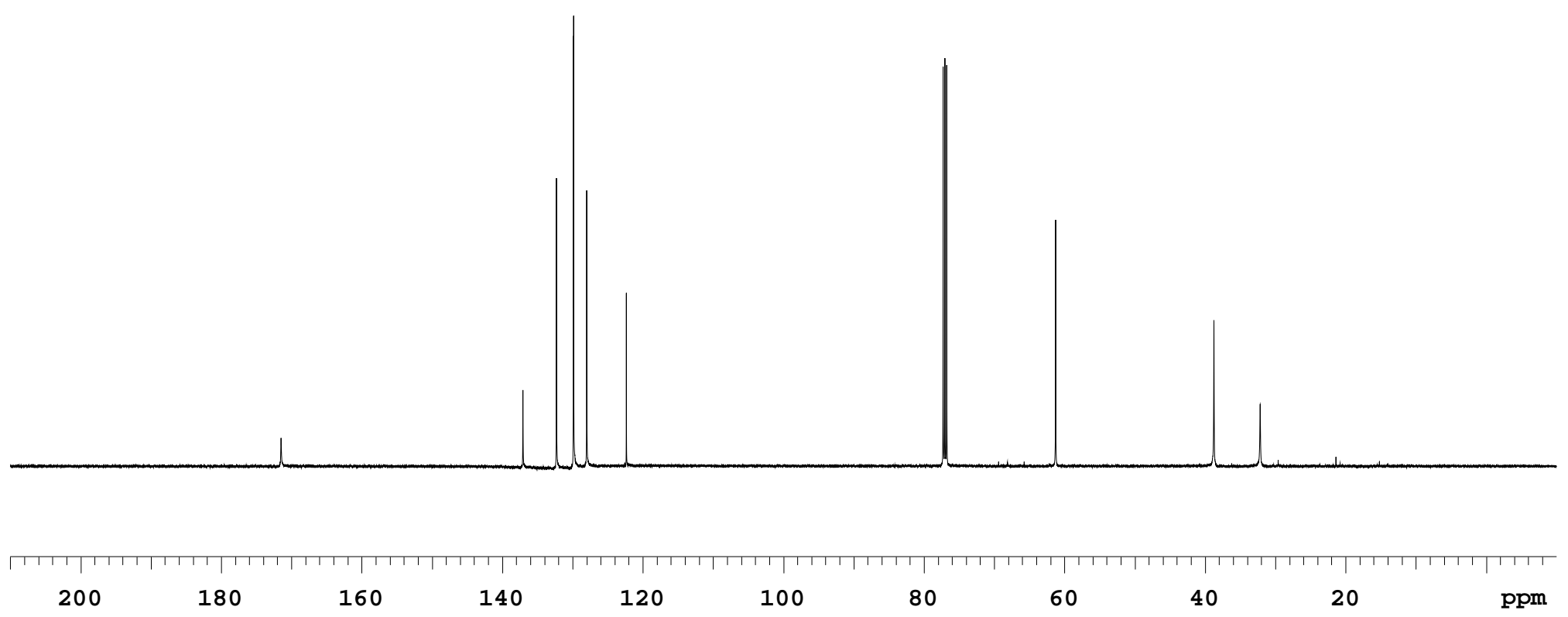

File: /mnt/d600/home13/rijnmr/hmrdata/DATA_FROM_NMRSERVICE/Patrick/2015.12/2015.12.02.u5_PM-06-041-4_loc4_14.10_C13_1D 
Department of Chemistry, University of Alberta

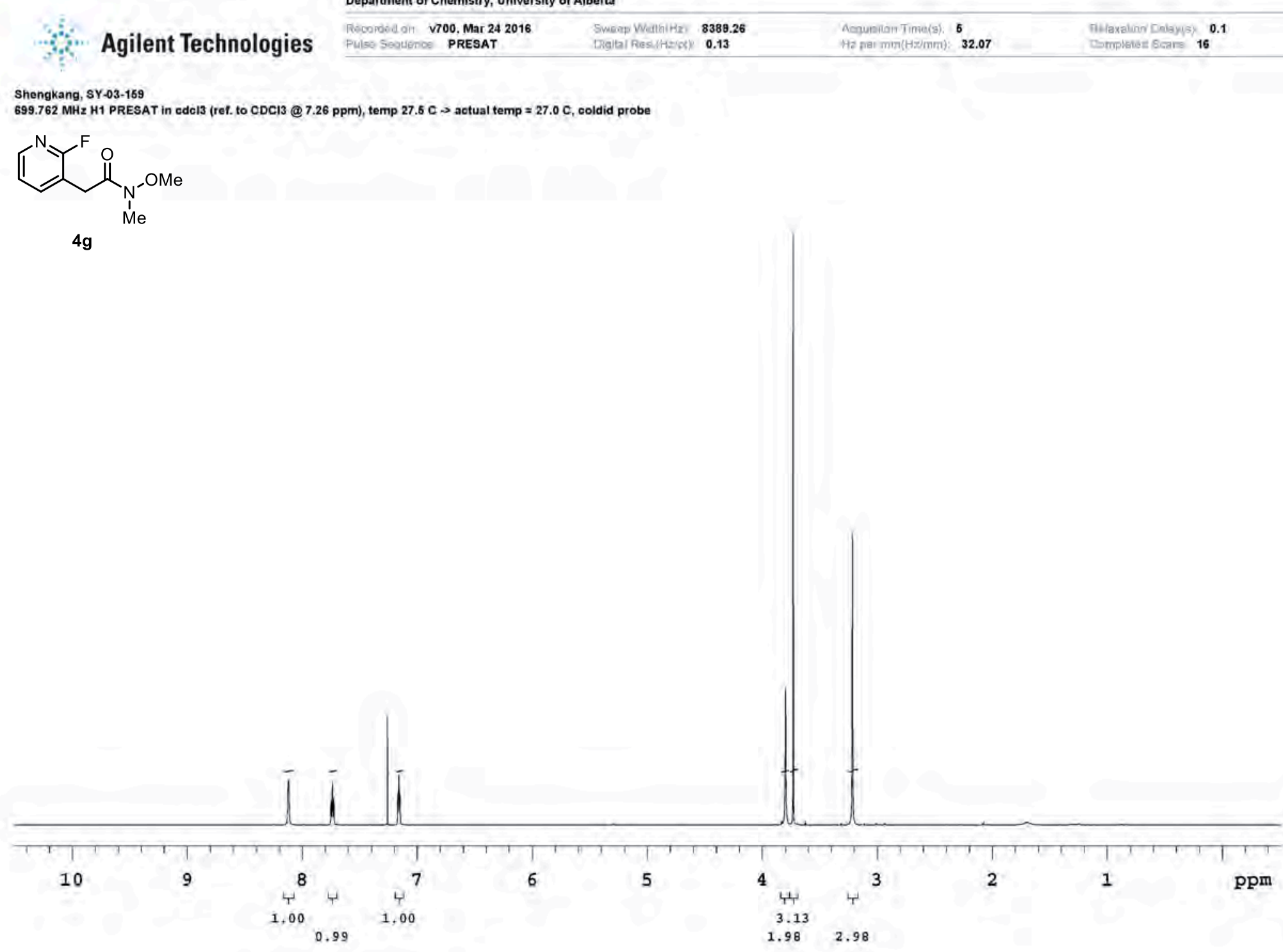




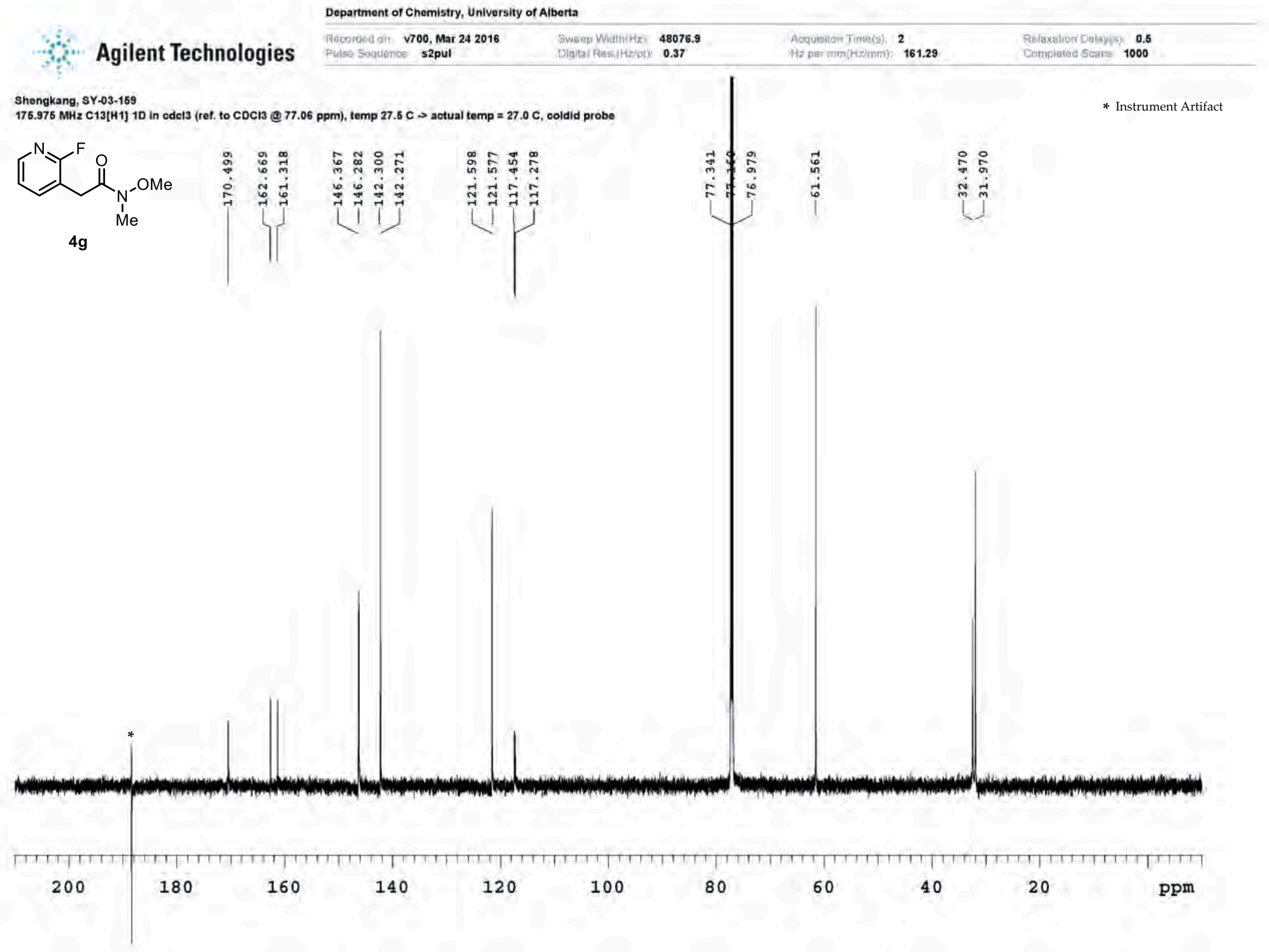


Department of Chemistry, University of Alberta

\begin{tabular}{|c|c|c|c|c|c|}
\hline Aqilent Technoloaies & 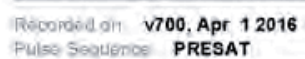 & 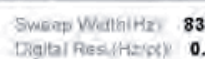 & $\begin{array}{l}3389.26 \\
0.13\end{array}$ & 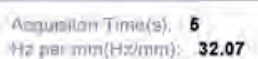 & 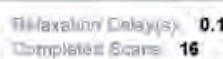 \\
\hline
\end{tabular}

Shengkang, SY-03-171-1

PRESAT in cdel3 (ret. to CDC13 @ $7.26 \mathrm{ppm}$ ), temp $27.5 \mathrm{C} \rightarrow$ actual temp $=27.0 \mathrm{C}$, ooldid probe

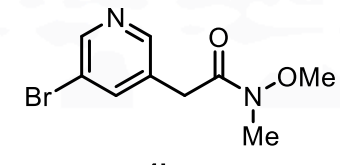

$4 \mathrm{~h}$

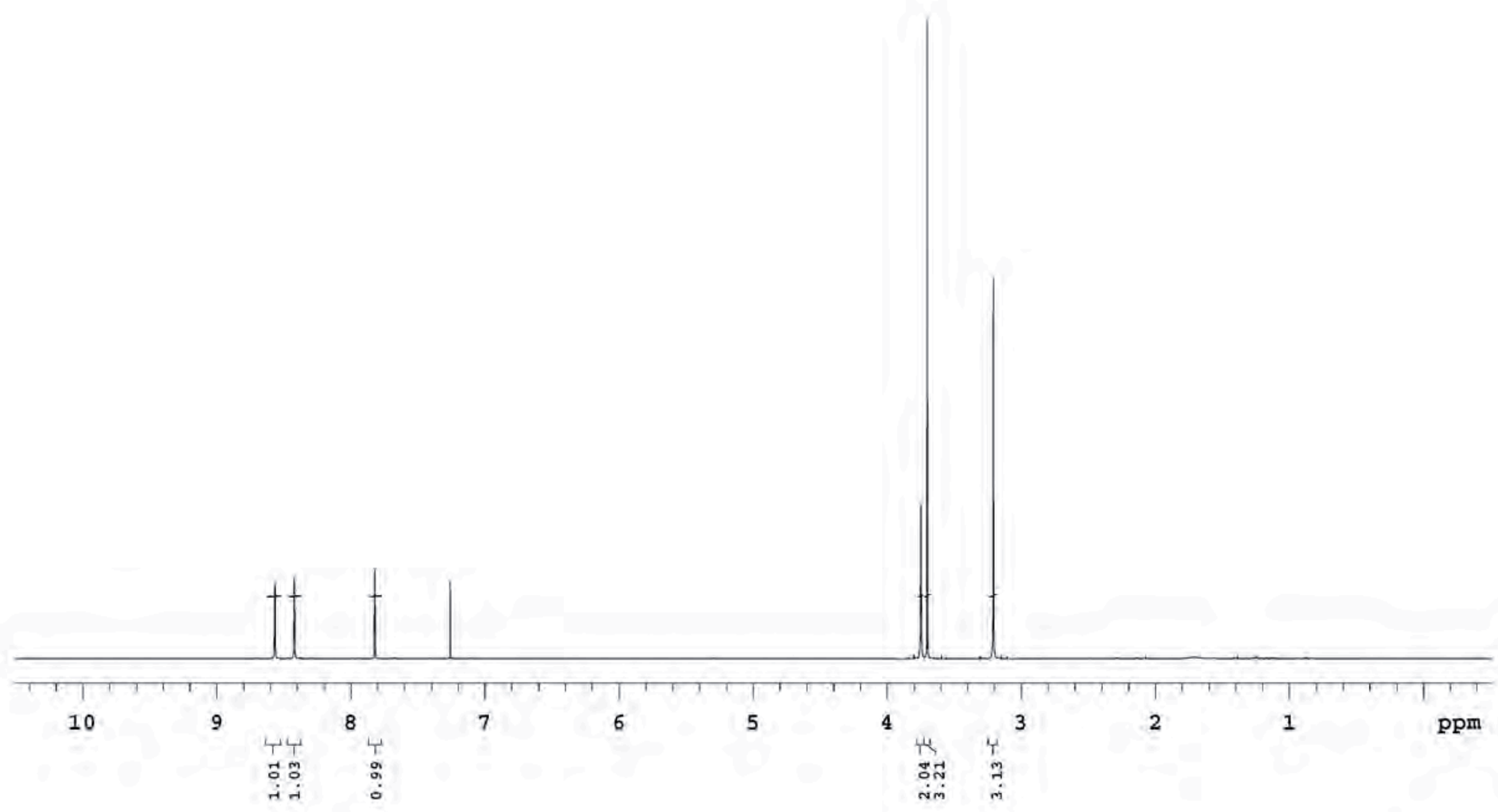




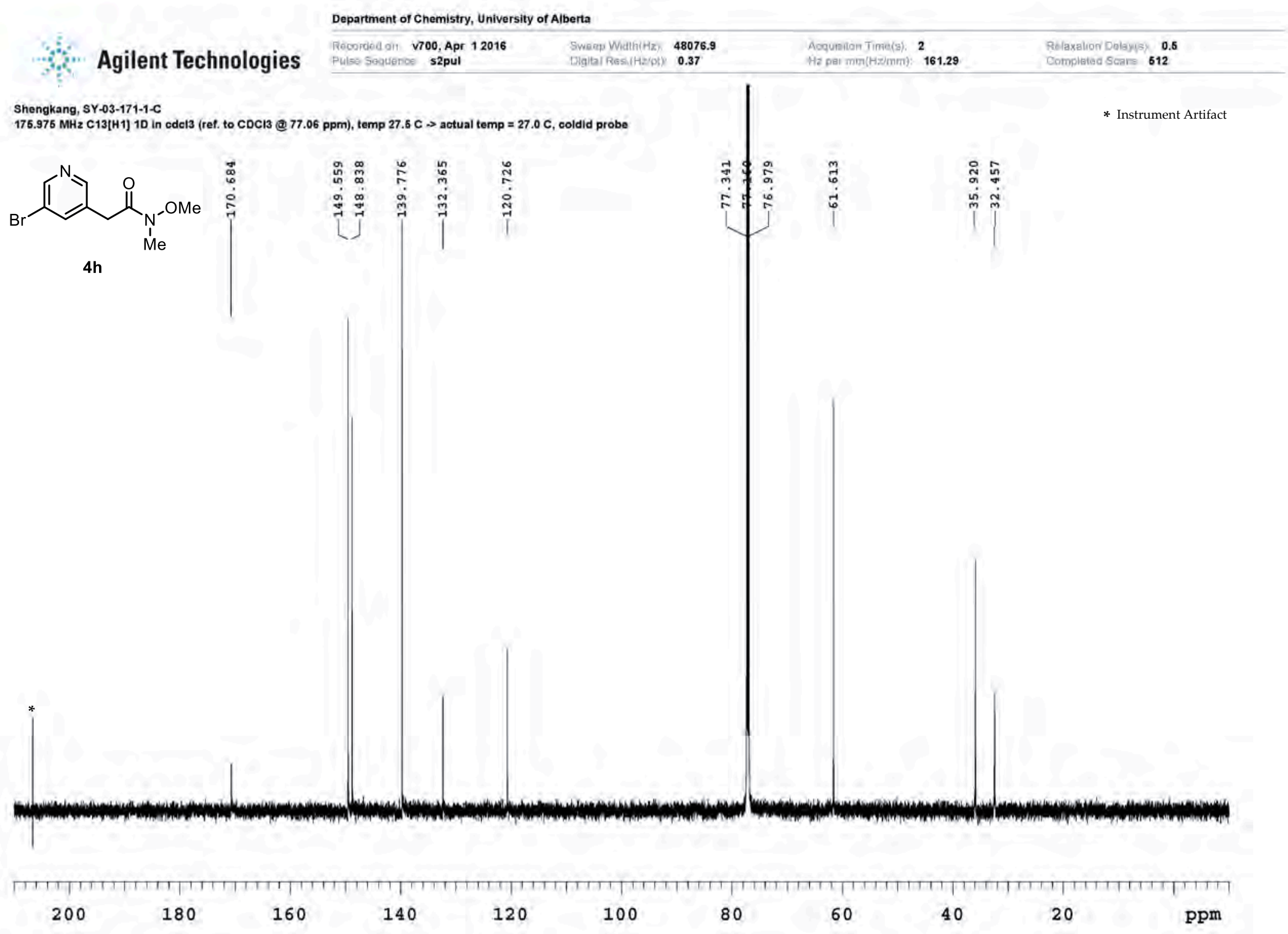


Department of Chemistry, University of Alberta
Agilent Technologies
Recorded on: ibd5, Sep 182016
Sweep Width(Hz): $\quad 6000.6$
Acquisiton Time(s): $\mathbf{5}$
Relaxation Delay(s): 0.1

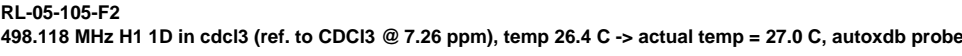

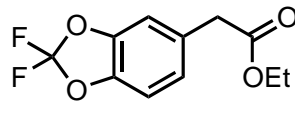

$5 a$

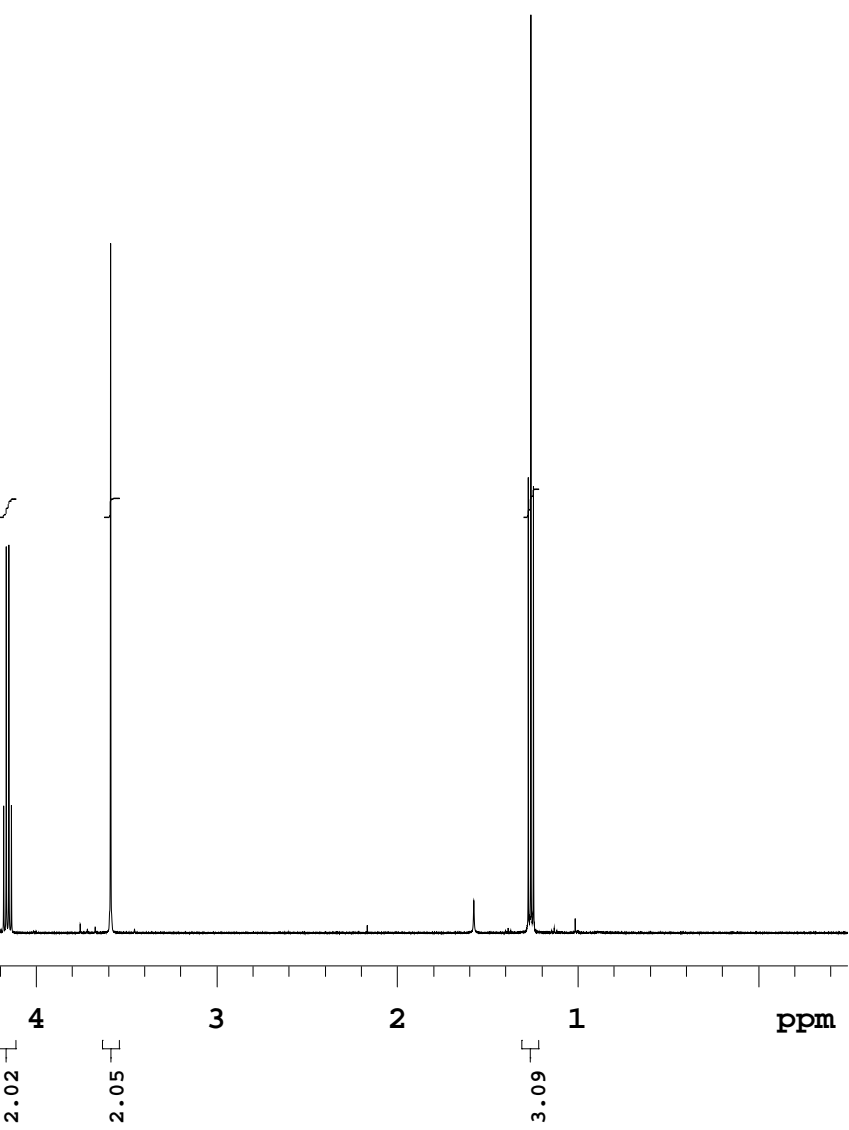

File:/mnt/d600/home13/rilnmr/nmrdata/Patrick/2016.09/2016.09.18 i5 RL-05-105-F2 H1_1D 
Department of Chemistry, University of Alberta

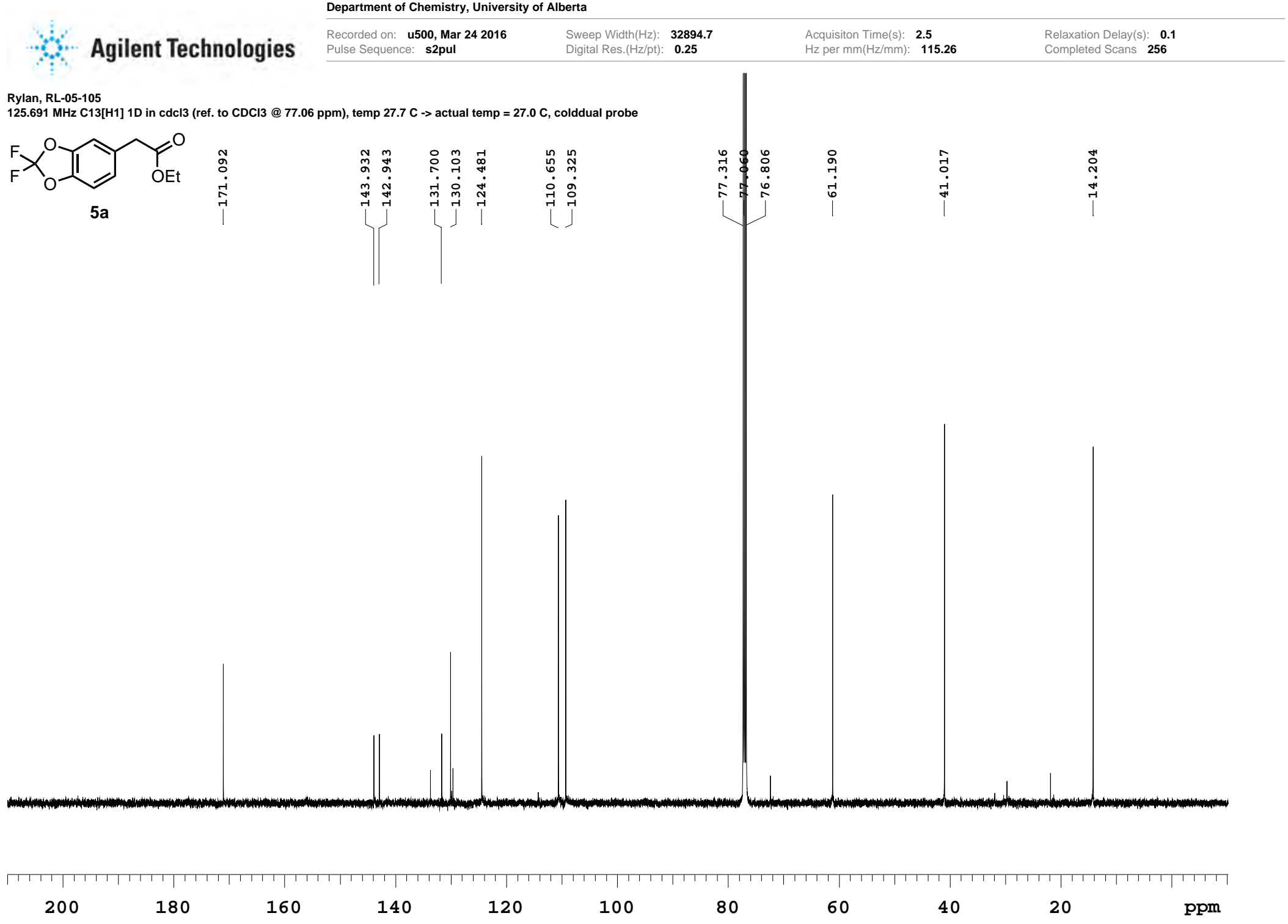

File: /mnt/d600/home13/rilnmr/nmrdata/DATA_FROM_NMRSERVICE/Rylan/2016.03/2016.03.24.u5_RL-05-105_loc1_17.24_C13_1D 
Department of Chemistry, University of Alberta

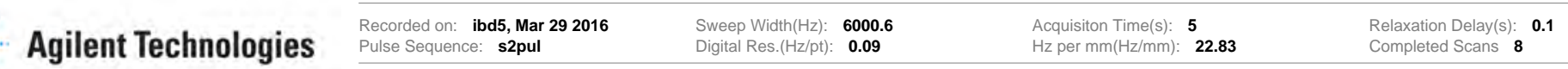

RL-05-119

498.118 MHz H1 1D in cdcl3 (ref. to CDCl3 @ 7.26 ppm), temp $26.4 \mathrm{C}>>$ actual temp = 27.0 C, autoxdb probe

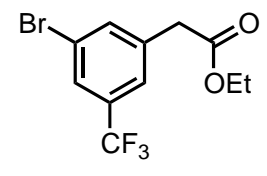

$5 b$

II I

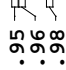

000

File: /mnt/d600/home13/rilnmr/nmrdata/Rylan/2016.03/2016.03.29.15_RL-05-119_H1_1D 
Department of Chemistry, University of Alberta

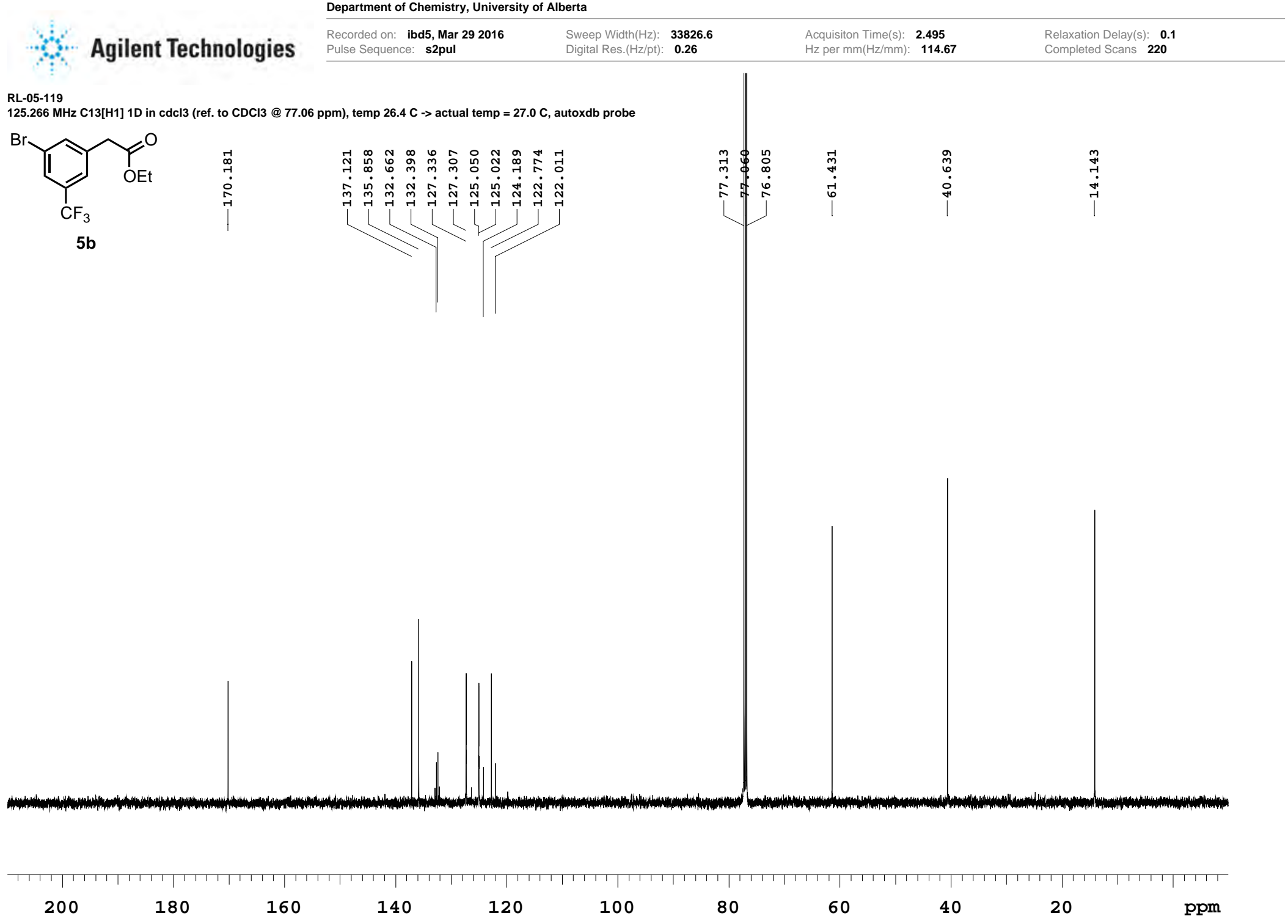

File: /mnt/d600/home13/rijnmr/hmrdata/Rylan/2016.03/2016.03.29.15_RL-05-119_C13_1D 
Department of Chemistry, University of Alberta

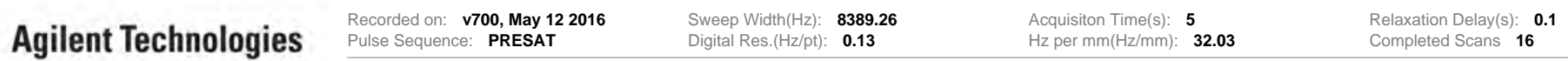

Rylan, RL-05-155-B-F2

699.762 MHz H1 PRESAT in cdcl3 (ref. to CDC13 @ 7.26 ppm), temp $27.5 \mathrm{C} \rightarrow$ actual temp $=27.0 \mathrm{C}$, coldid probe

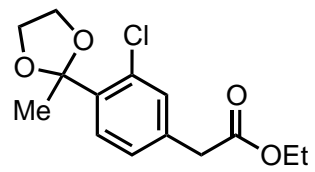

$5 d$

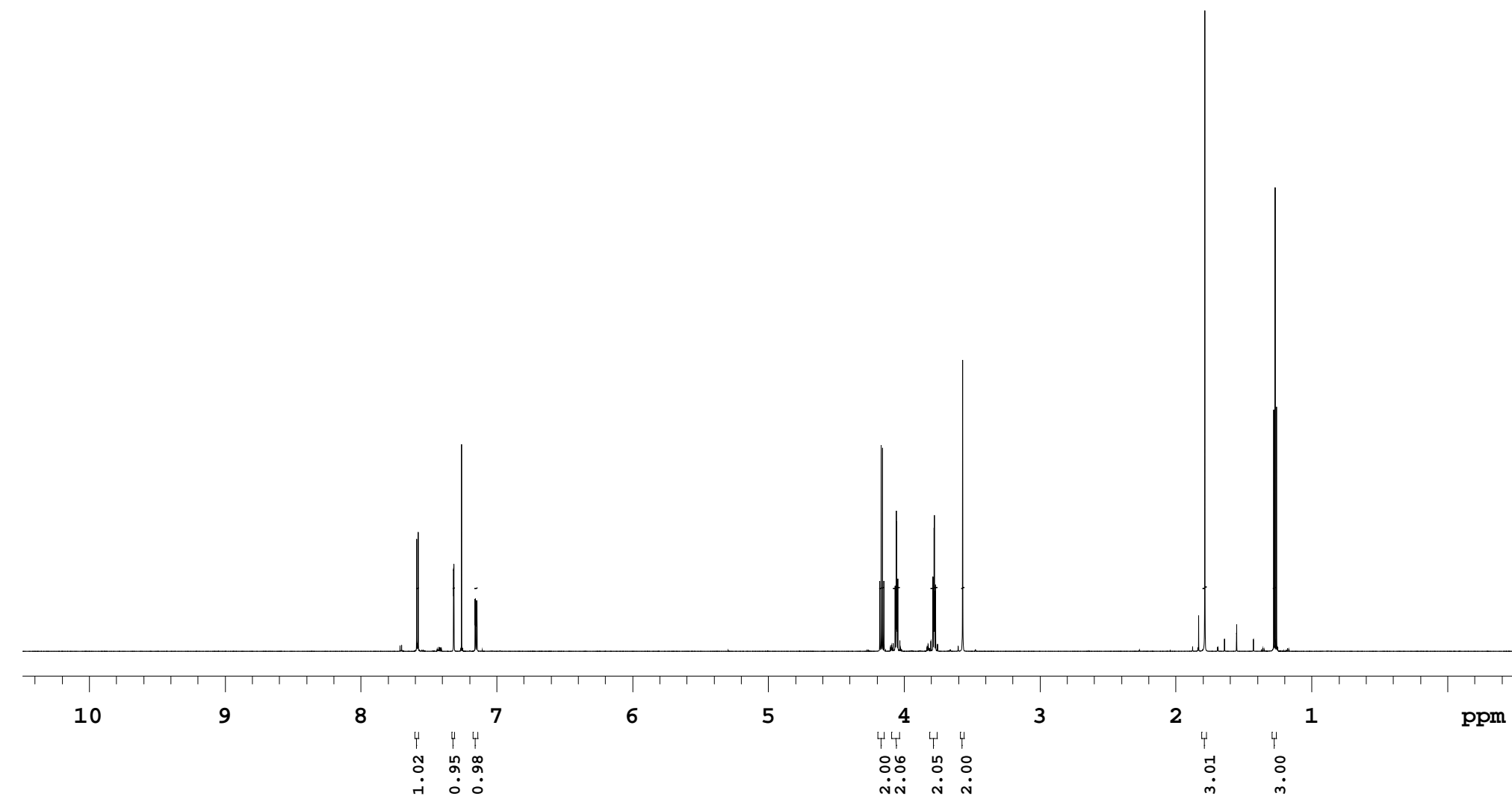

File: /mnt/d600/home13/rilnmr/nmrdata/DATA_FROM_NMRSERVICE/Rylan/2016.05/2016.05.12.v7_RL-05-155-B-F2_loc14_15.50_H1_1D 
Department of Chemistry, University of Alberta

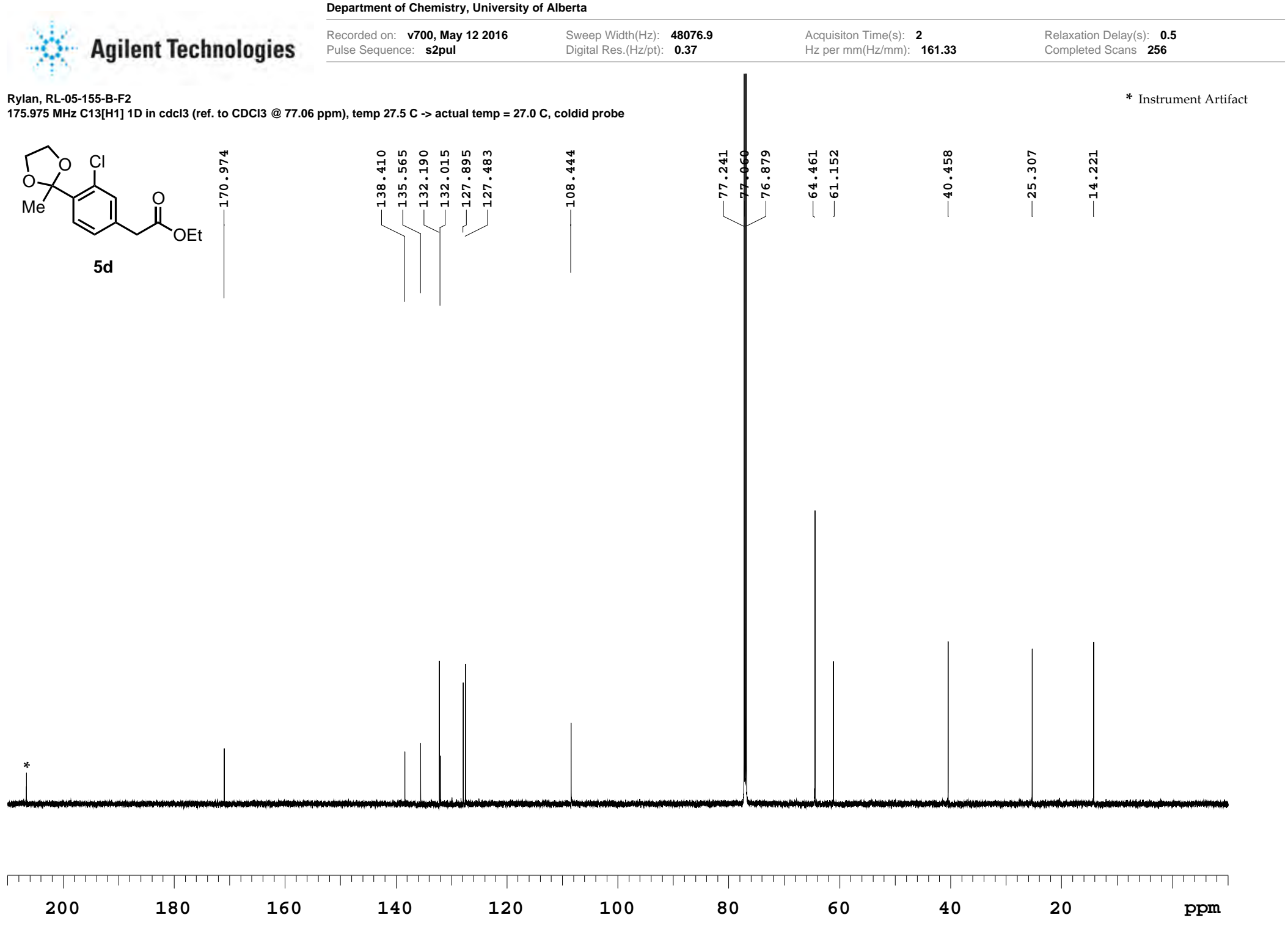

File: /mnt/d600/home13/rilnmr/nmrdata/DATA_FROM_NMRSERVICE/Rylan/2016.05/2016.05.12.v7_RL-05-155-B-F2_loc14_15.52_C13_1D 
Department of Chemistry, University of Alberta

\begin{tabular}{|c|c|c|c|c|c|}
\hline Aailent Technoloaies & $\begin{array}{l}\text { Recorded on: u500, May } 232016 \\
\text { Pulse Sequence: PRESAT }\end{array}$ & $\begin{array}{l}\text { Sweep Width }(\mathrm{Hz}): \mathbf{6 0} \\
\text { Digital Res. }(\mathrm{Hz} / \mathrm{pt}): \mathbf{0 .}\end{array}$ & $\begin{array}{l}6009.62 \\
0.09\end{array}$ & $\begin{array}{l}\text { Acquisiton Time }(\mathrm{s}): \quad \mathbf{5} \\
\mathrm{Hz} \text { per } \mathrm{mm}(\mathrm{Hz} / \mathrm{mm}): \quad \mathbf{2 2 . 9 1}\end{array}$ & $\begin{array}{l}\text { Relaxation Delay(s): } \mathbf{0} \\
\text { Completed Scans } \mathbf{1 6}\end{array}$ \\
\hline
\end{tabular}

Wenyu, RL-05-157-final-product

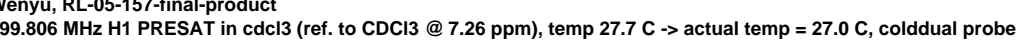

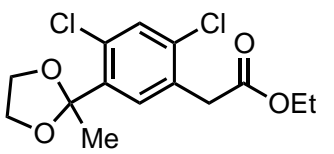

$5 e$

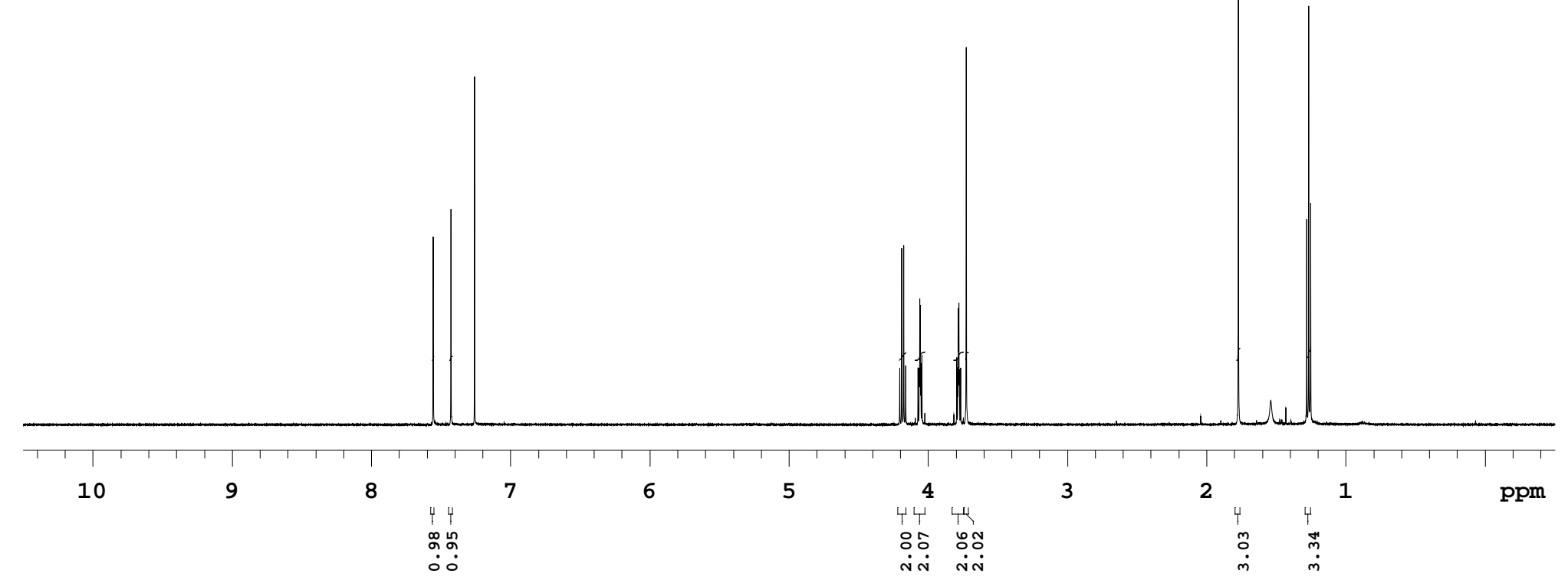

File: /mnt/d600/home13/rilnmr/nmrdata/DATA_FROM_NMRSERVICE/Wenyu/2016.05/2016.05.23.u5_RL-05-157-final-product_loc7_11.29_H1_1D 
Department of Chemistry, University of Alberta

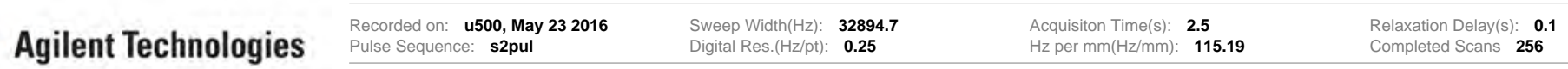

Wenyu, RL-05-157-final-product

作

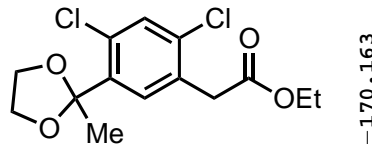

$5 e$

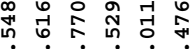

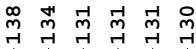

LLL

$\stackrel{\text { ก }}{\text { ำ }}$

\begin{tabular}{|c|c|c|c|}
\hline 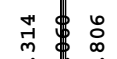 & ભ్ & $\stackrel{2}{?}$ & $\stackrel{\circ}{\stackrel{\circ}{0}}$ \\
\hline & தं & 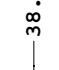 & $\stackrel{\text { ஸे }}{\mid}$ \\
\hline
\end{tabular}

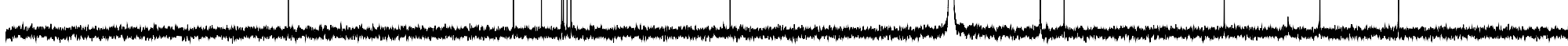
200
180
160
140
120
100
80
60
40
20
ppm 
Department of Chemistry, University of Alberta

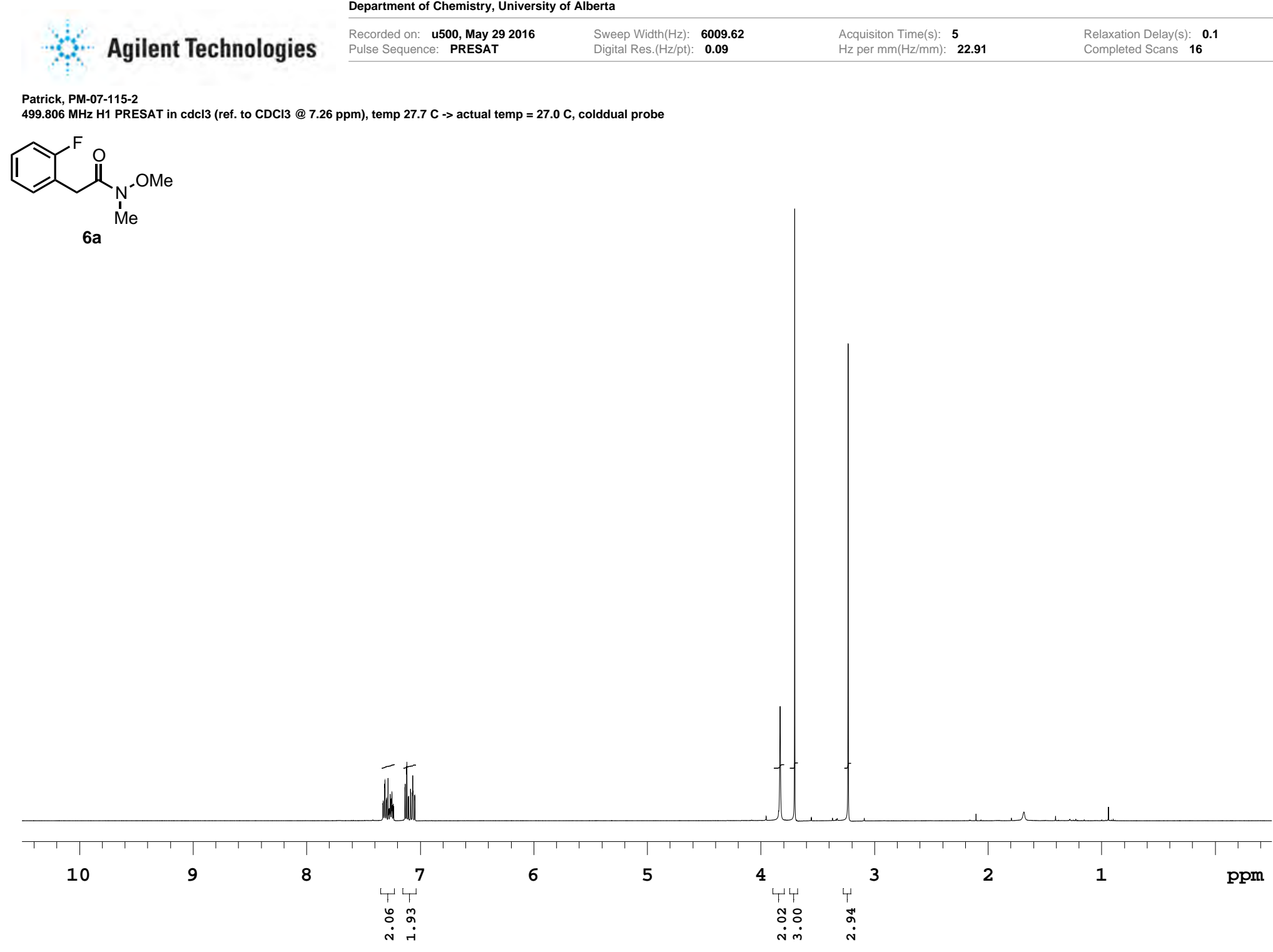

File:/mnt/d600/home12/gennmr/nmrdata/LUNDGREN/Patrick/2016.05/2016.05.29.u5_PM-07-115-2_loc3_19.30_H1_1D 


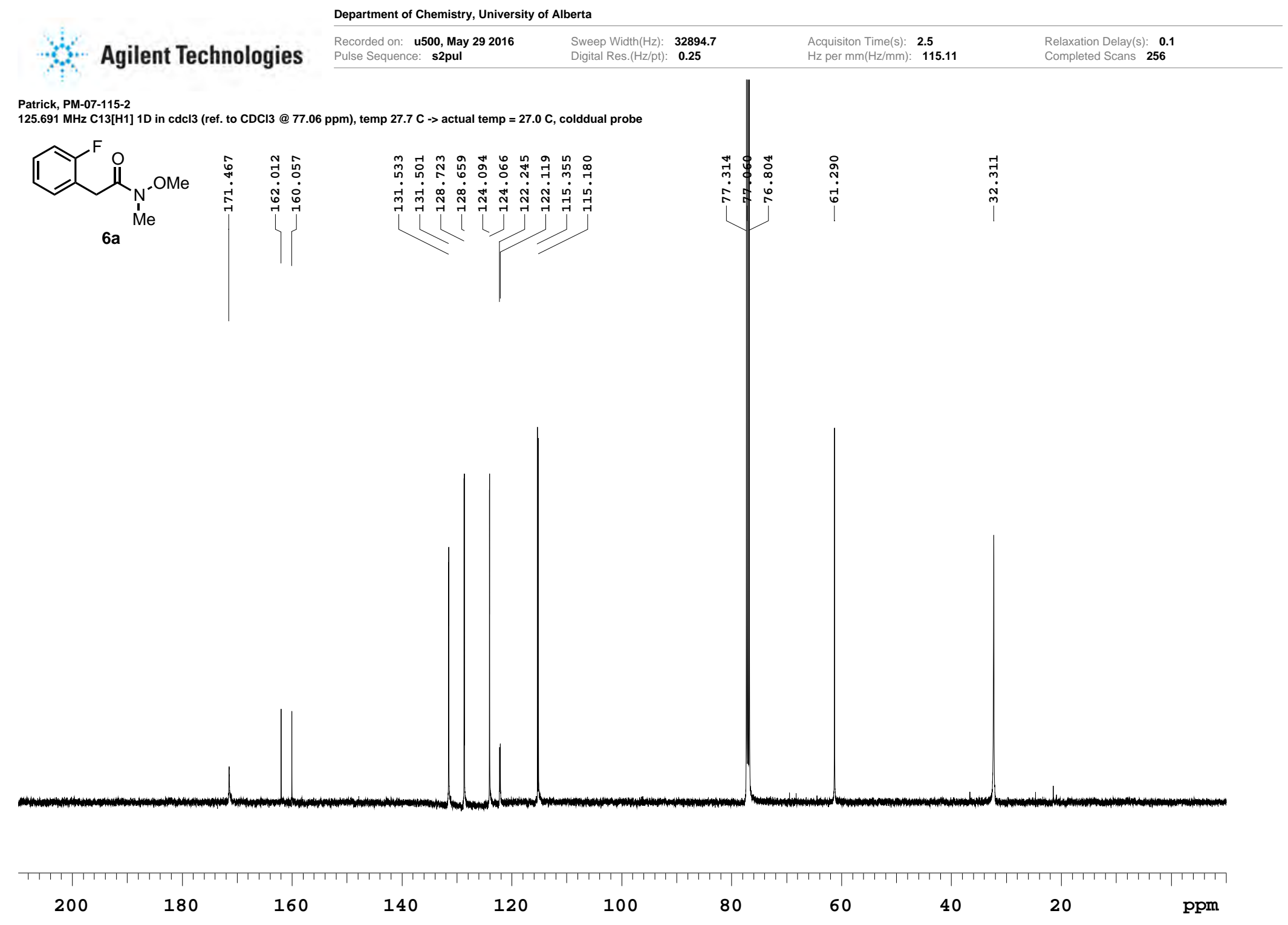

File: /mnt/d600/home12/gennmr//mmrdata/LUNDGREN/Patrick/2016.05/2016.05.29.u5_PM-07-115-2_loc3_19.32_C13_1D 
Department of Chemistry, University of Alberta
Agilent Technologies
Recorded on: ibd5, Sep 212016
$\begin{array}{ll}\text { Sweep Width }(\mathrm{Hzz}) & \mathbf{6 0 0 0 . 6} \\ \text { Digital Res. }(\mathrm{Hz} / \mathrm{pt}): & \mathbf{0 . 0 9}\end{array}$
Acquisiton Time(s): $\mathbf{5}$
Relaxation Delay(s): 0.1

WQ-01-043-F6
498.118 MHz H1 1D in cdcl3 (ref. to CDCl3 @ 7.26 ppm), temp 26.4 C $>$ actual temp = 27.0 C, autoxdb probe

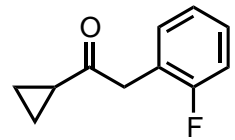

6a'

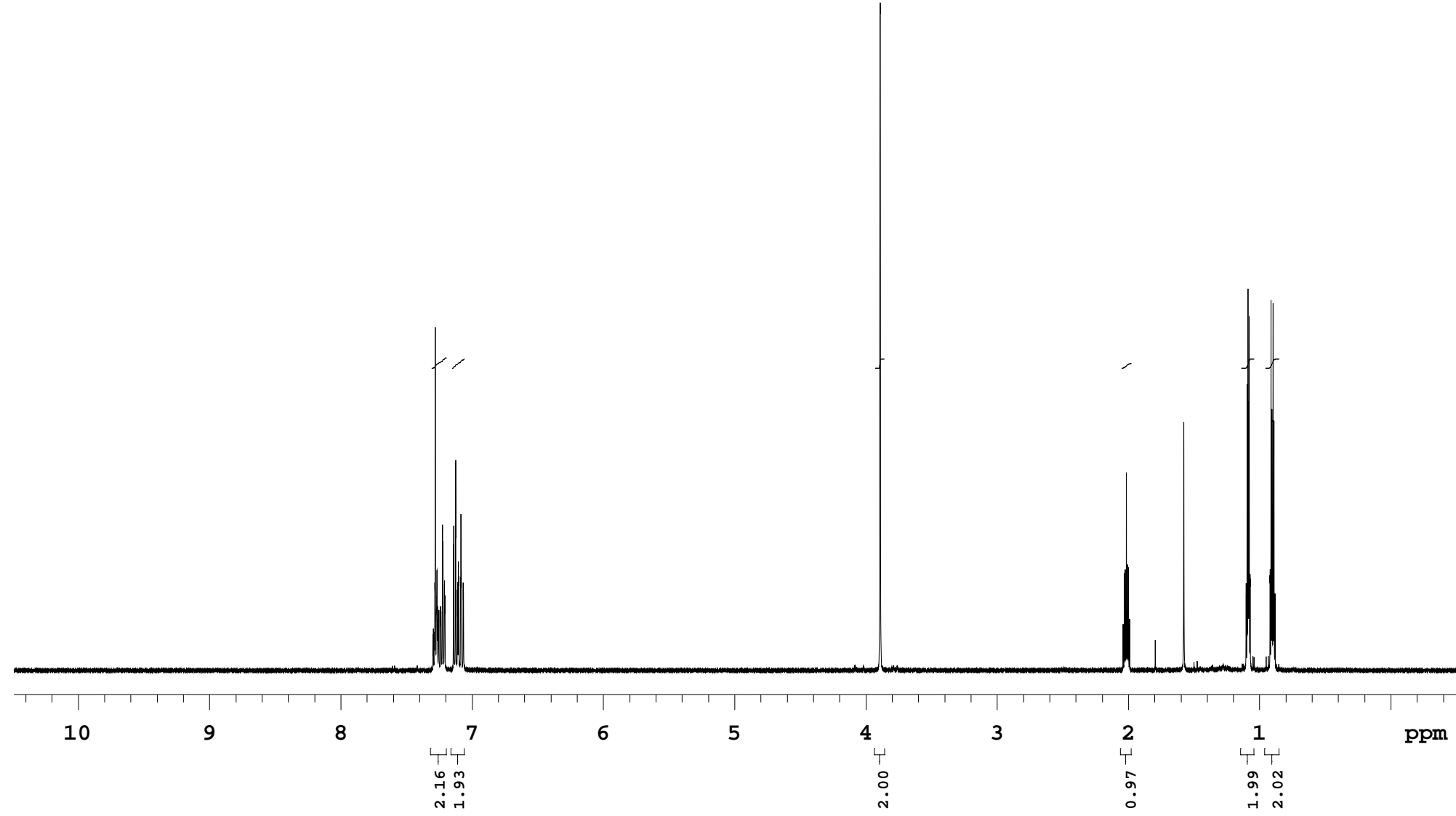

File: /mnt/d600/home13/rillnmr/nmrdata/Patrick/2016.09/2016 09.21 i5 WQ-01-043-F6 H1 1D 
Department of Chemistry, University of Alberta

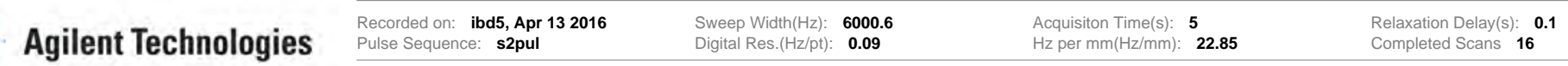

PM-07-113-5

498.118 MHz H1 1D in cdCl3 (ref. to CDCl3 @ 7.26 ppm), temp 26.4 C $\rightarrow$ actual temp = 27.0 C, autoxdb probe

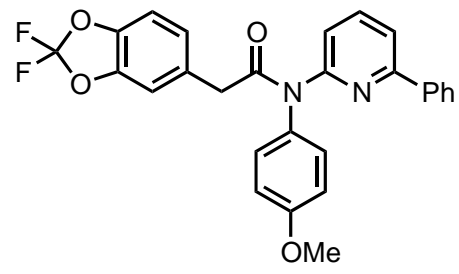

$6 b$

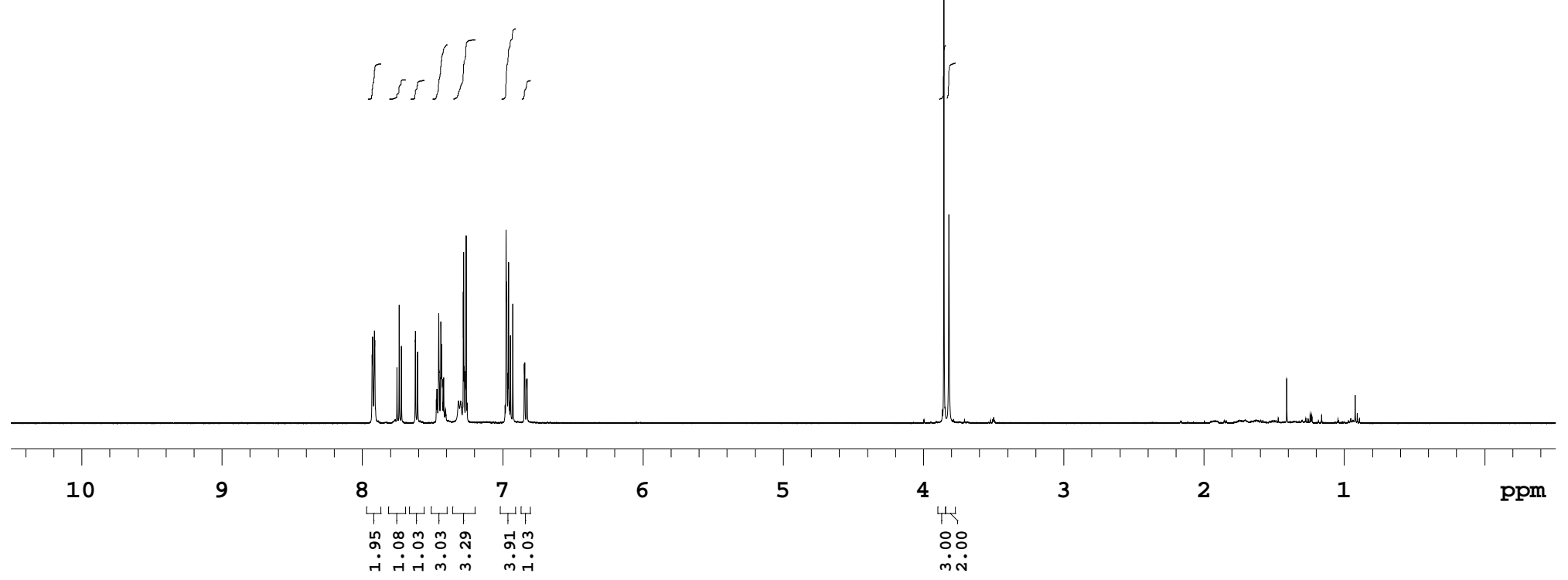

File: /mnt/d600/home13/rijnmr/nmrdata/Patrick/2016.04/2016.04.13.15_PM-07-113-5_H1_1D 
Department of Chemistry, University of Alberta

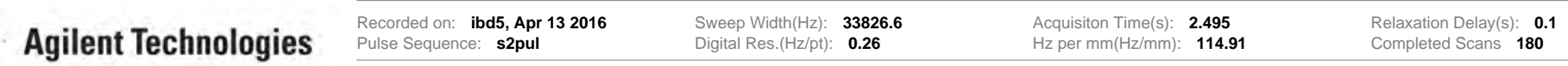

$125.266 \mathrm{MHz} C 13[\mathrm{H} 1] 1 \mathrm{D}$ in cdcl3 (ref. to $\mathrm{CDCl} 3 @ 77.06 \mathrm{ppm}$ ), temp $26.4 \mathrm{C}->$ actual temp $=27.0 \mathrm{C}$, autoxdb probe

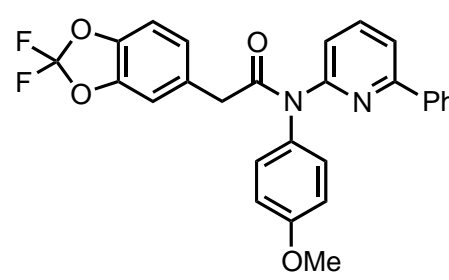

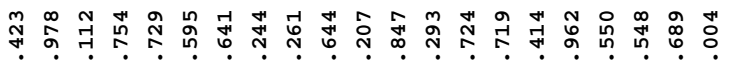

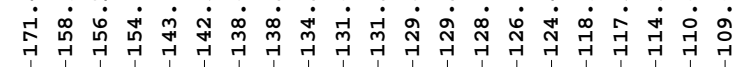

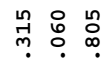

iิ

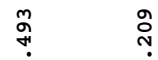

$6 b$

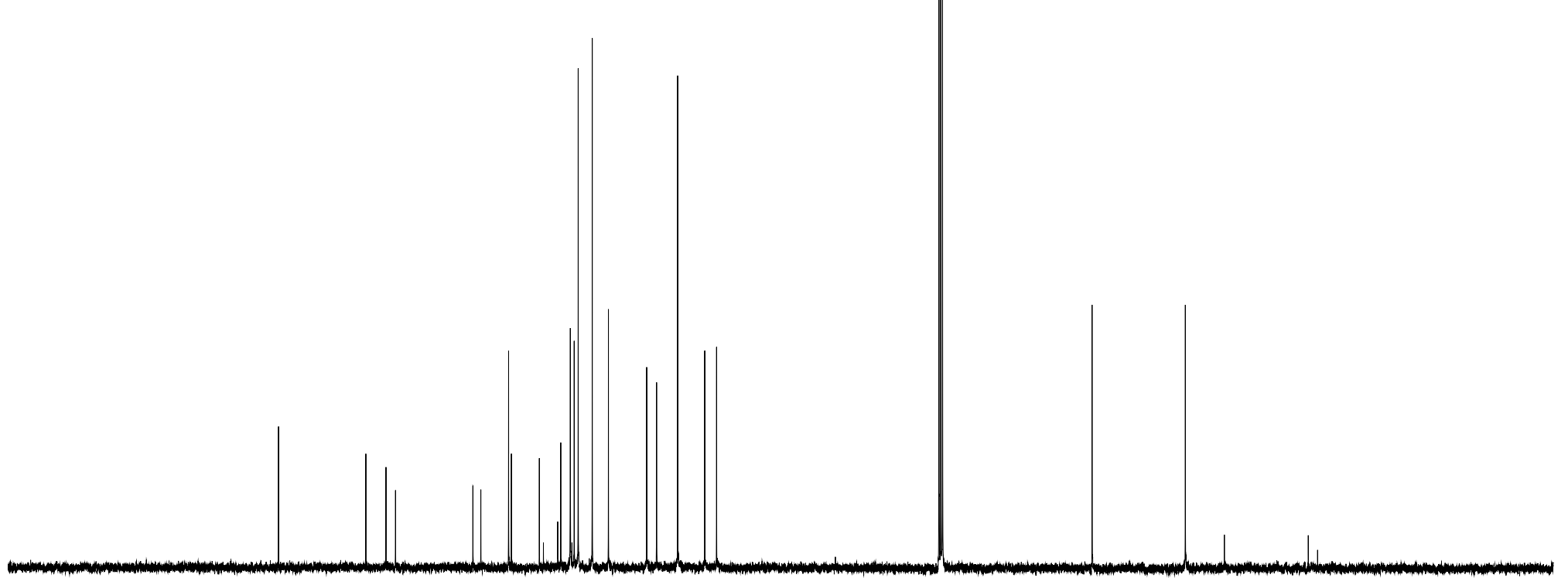

200

180

160

140

120

100

80

60

40

20

ppm 
Department of Chemistry, University of Alberta

\begin{tabular}{lllll}
\multirow{2}{*}{ Agilent Technologies } & Recorded on: v700, Jun $\mathbf{2} 2 \mathbf{2 0 1 6}$ & Sweep Width(Hz): $\mathbf{8 3 8 9 . 2 6}$ & Acquisiton Time(s): $\mathbf{5}$ & Relaxation Delay(s): $\mathbf{0 . 1}$ \\
\cline { 2 - 5 } & Pulse Sequence: PRESAT & Digital Res.(Hz/pt): $\mathbf{0 . 1 3}$ & Hz per mm(Hz/mm): $\mathbf{3 2 . 1 1}$ & Completed Scans $\mathbf{1 6}$
\end{tabular}

Patrick, PM-07-205-B-C2-F7-10-3

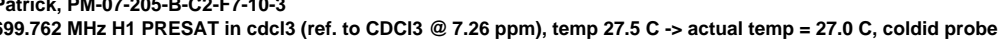

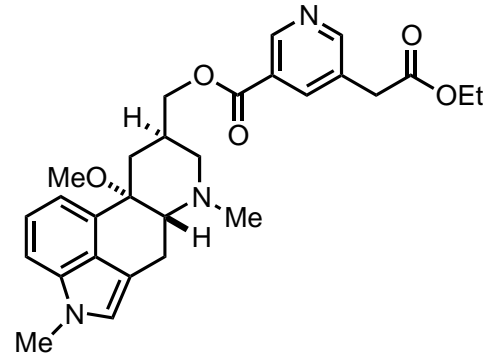

$7 a$

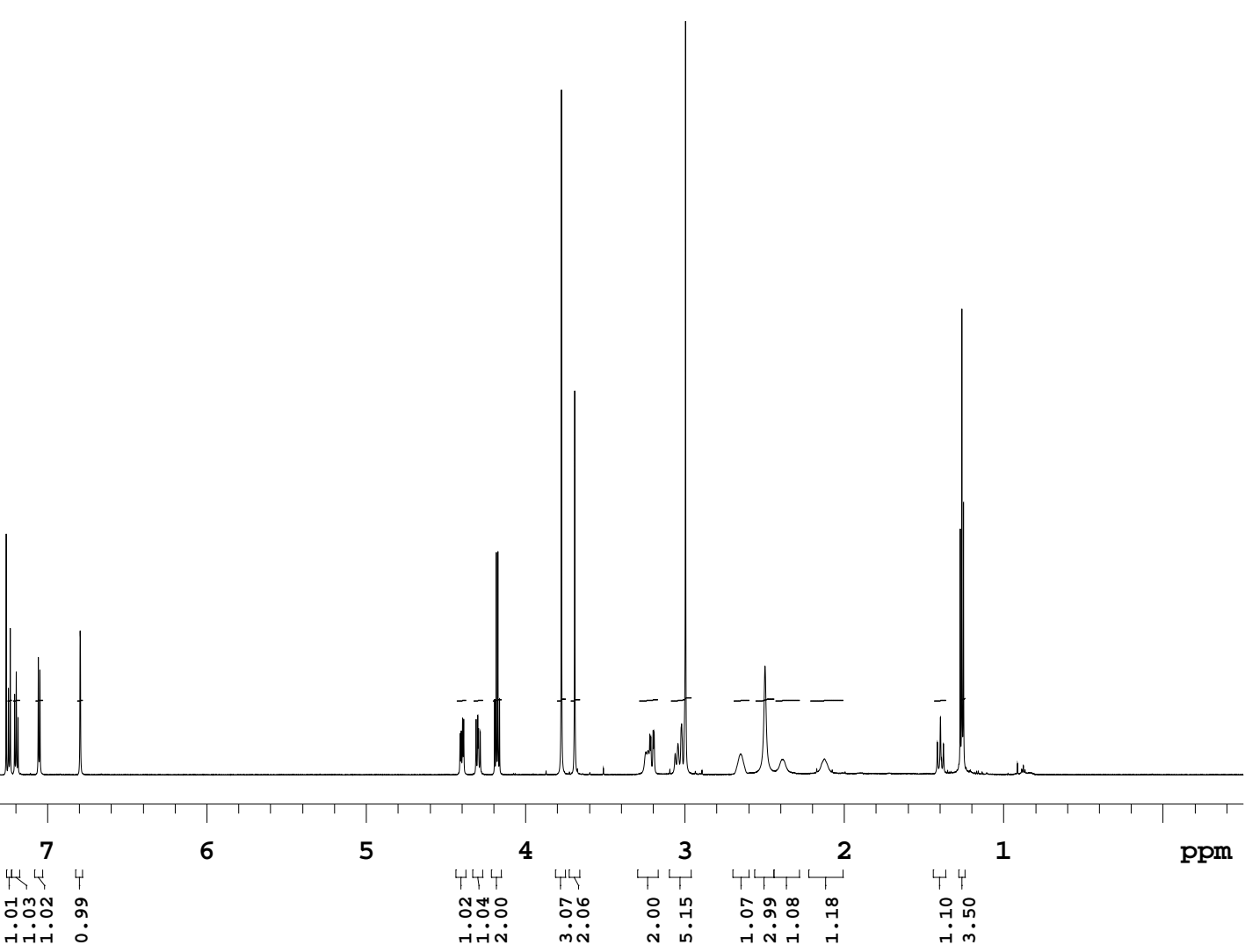

File: /mnt/d600/home13/rilnmr//mmrdata/DATA_FROM_NMRSERVICE/Patrick/2016.06/2016.06.2.v7_PM-07-205-B-C2-F7-10-3_loc31_10.55_H1_1D 
Department of Chemistry, University of Alberta

\begin{tabular}{|c|c|c|c|c|}
\hline Aqilent Technoloqies & $\begin{array}{l}\text { Recorded on: u500, Jun } 22016 \\
\text { Pulse Sequence: s2pul }\end{array}$ & $\begin{array}{l}\text { Sweep Width(Hz): } \quad \mathbf{3 2 8 9 4 . 7} \\
\text { Digital Res.(Hz/pt): } \mathbf{0 . 2 5}\end{array}$ & $\begin{array}{l}\text { Acquisiton Time(s): } \\
\text { Hz per } \mathrm{mm}(\mathrm{Hz} / \mathrm{mm}): \mathbf{1 1 5 . 1}\end{array}$ & $\begin{array}{l}\text { Relaxation Delay(s): } 0.1 \\
\text { Completed Scans } 256\end{array}$ \\
\hline
\end{tabular}

Patrick, PM-07-205-B-F7-10-4

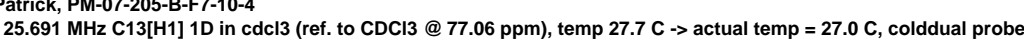

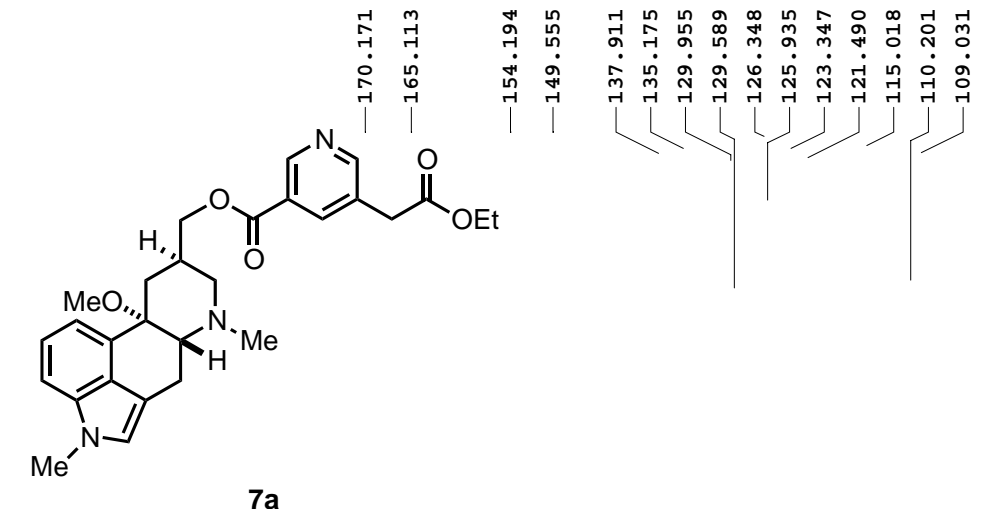

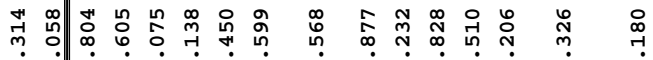

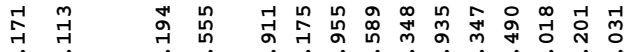

号

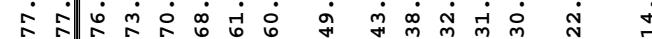

ล

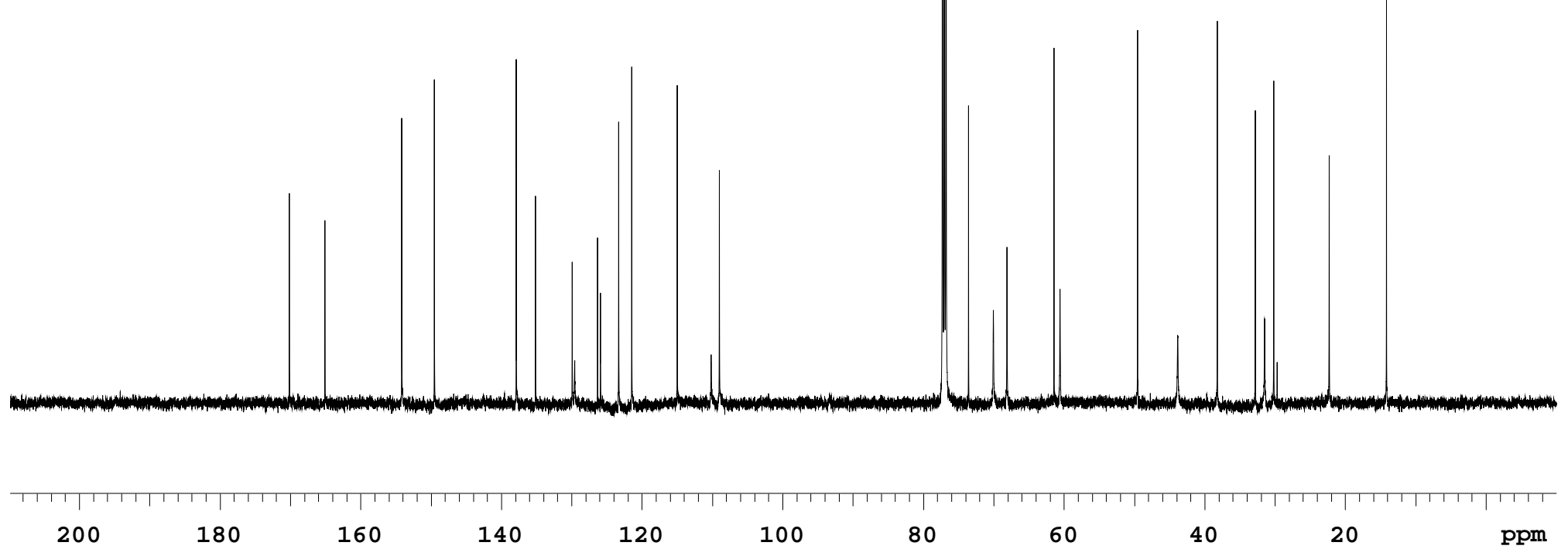


Department of Chemistry, University of Alberta

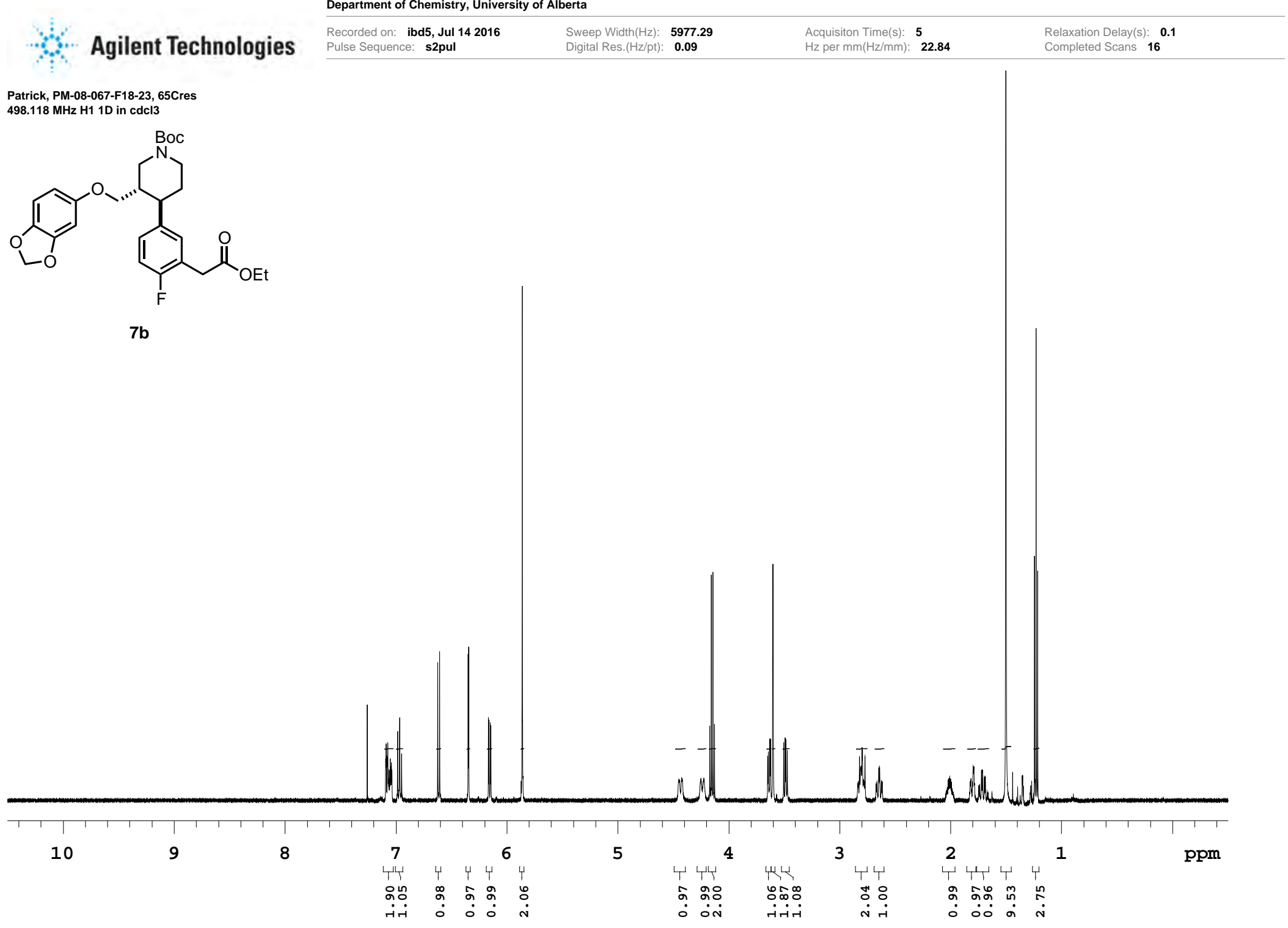

File: /mnt/d600/home13/jilnmr/nmrdata/DATA_FROM_NMRSERVICE/Patrick/2016.07/2016.07.14.15_PM-08-067-F18-23_65C_H1_1D 
Department of Chemistry, University of Alberta

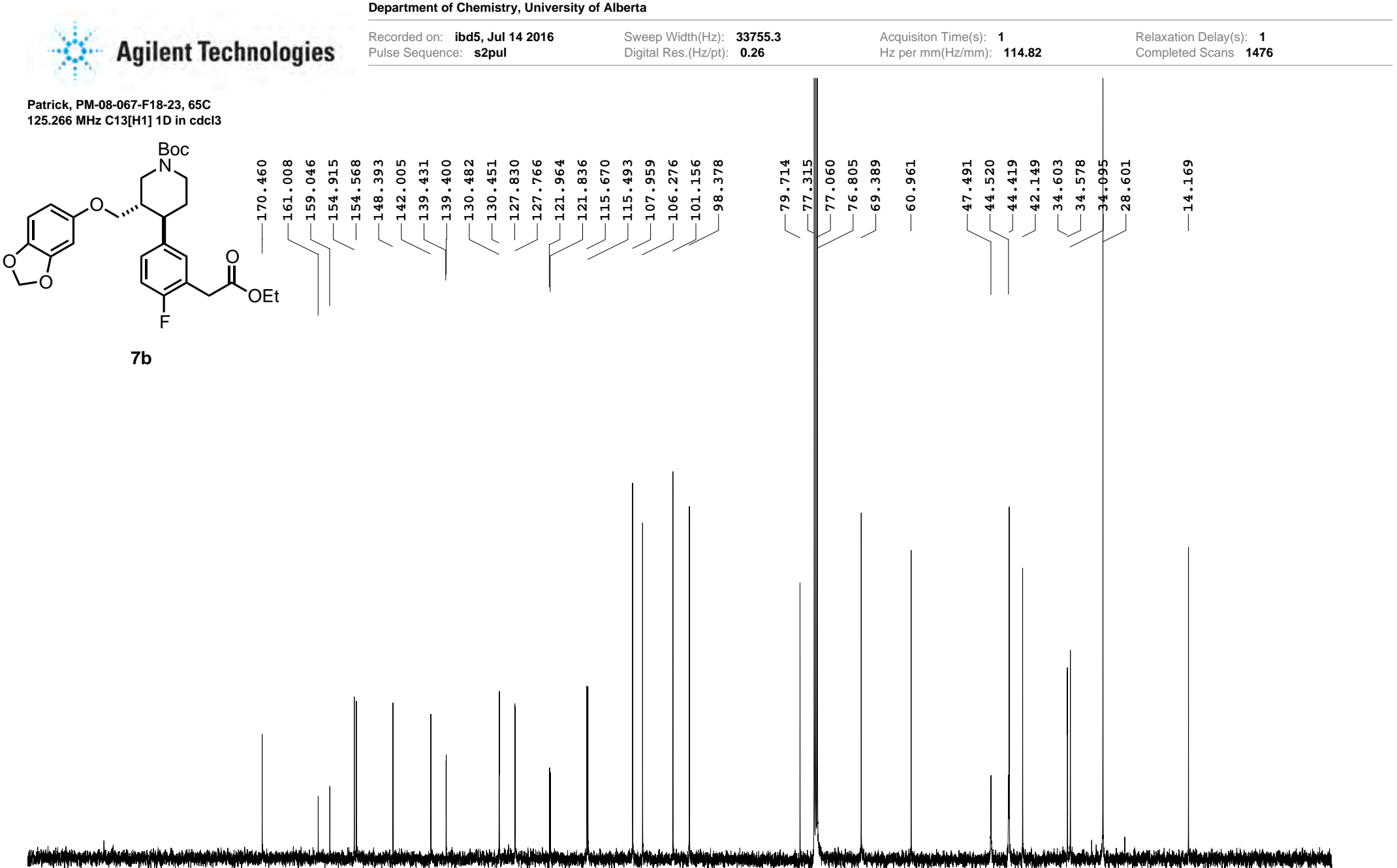

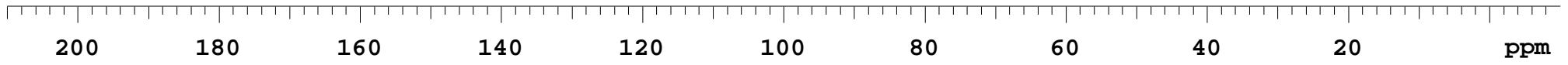


Department of Chemistry, University of Alberta

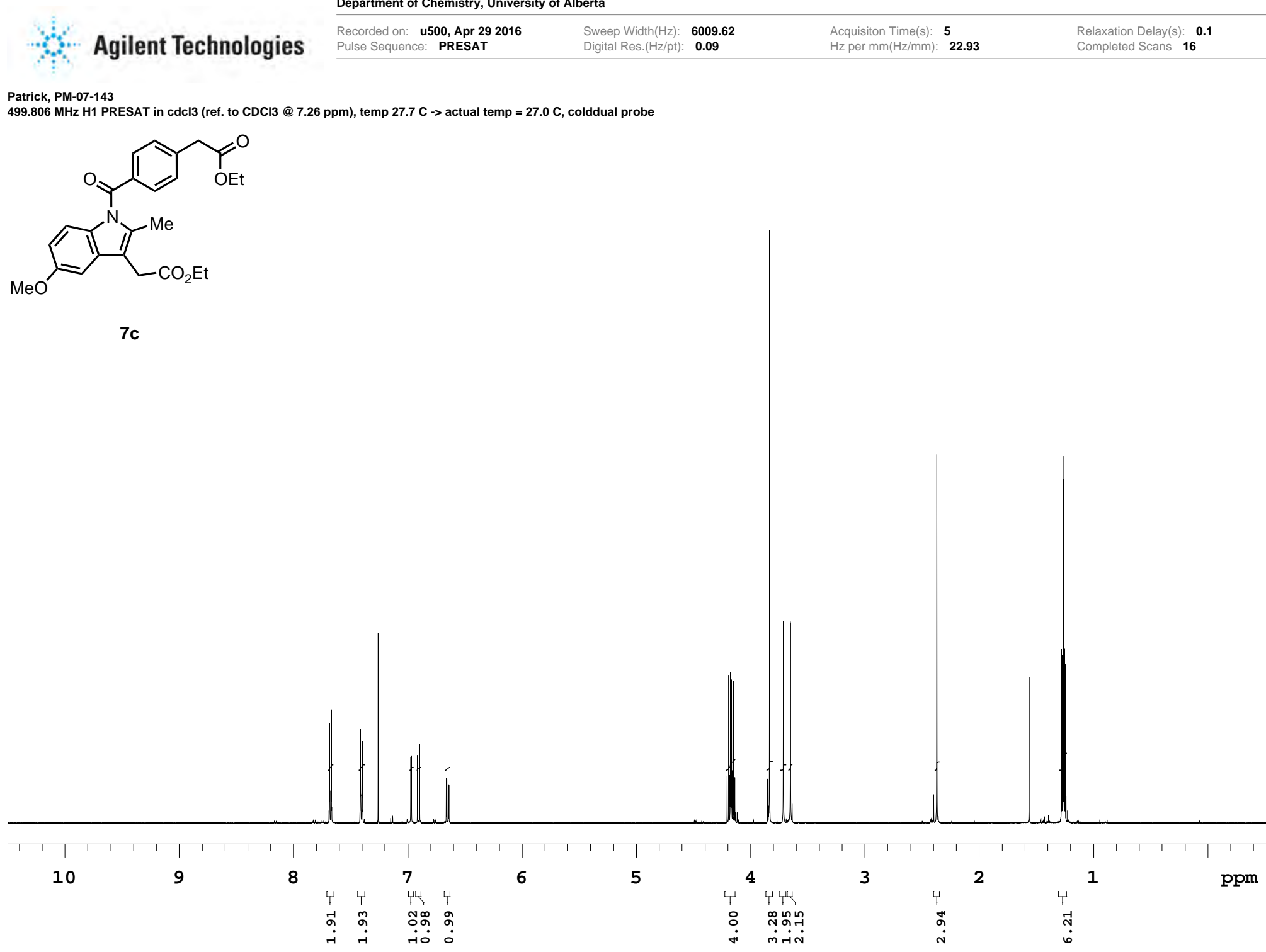

File: /mnt/d600/home13/jilnmr/nmrdata/DATA_FROM_NMRSERVICE/Patrick/2016.04/2016.04.29.u5_PM-07-143_loc5_14.09_H1_1D 
Department of Chemistry, University of Alberta

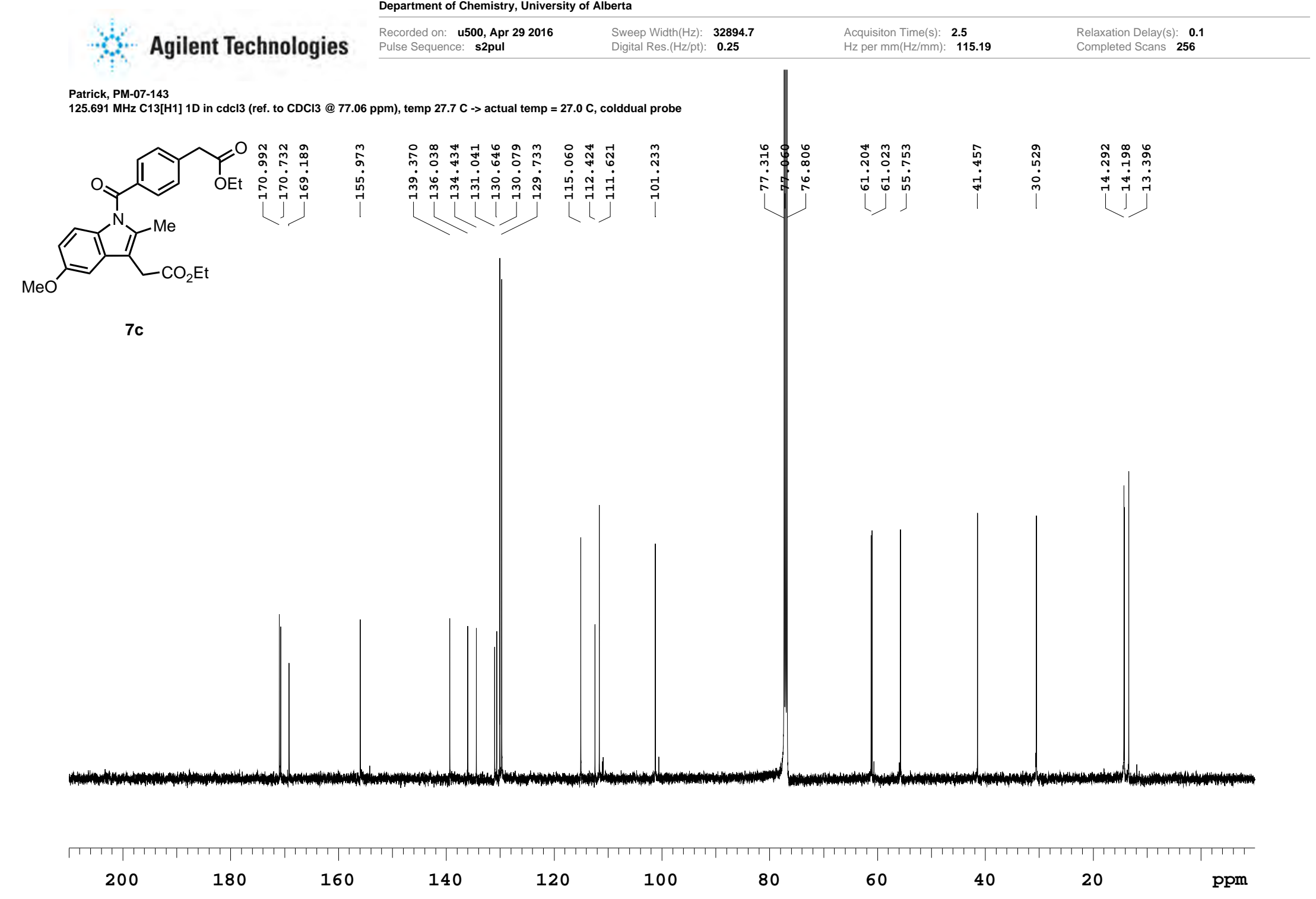

File: /mnt/d600/home13/rilnmr/nmmrdata/DATA_FROM_NMRSERVICE/Patrick/2016.04/2016.04.29.u5_PM-07-143_loc5_14.11_C13_1D 
Department of Chemistry, University of Alberta

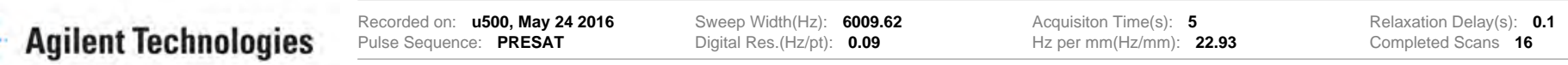

Patrick, PM-07-193-F20-27
499.806 MHz H1 PRESAT in cdcl3 (ref. to CDC13 @ 7.26 ppm), temp 27.7 C $>$ actual temp $=27.0$ C, colddual probe

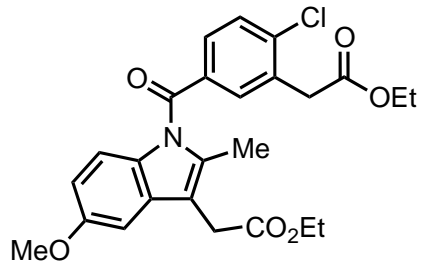

$7 d$

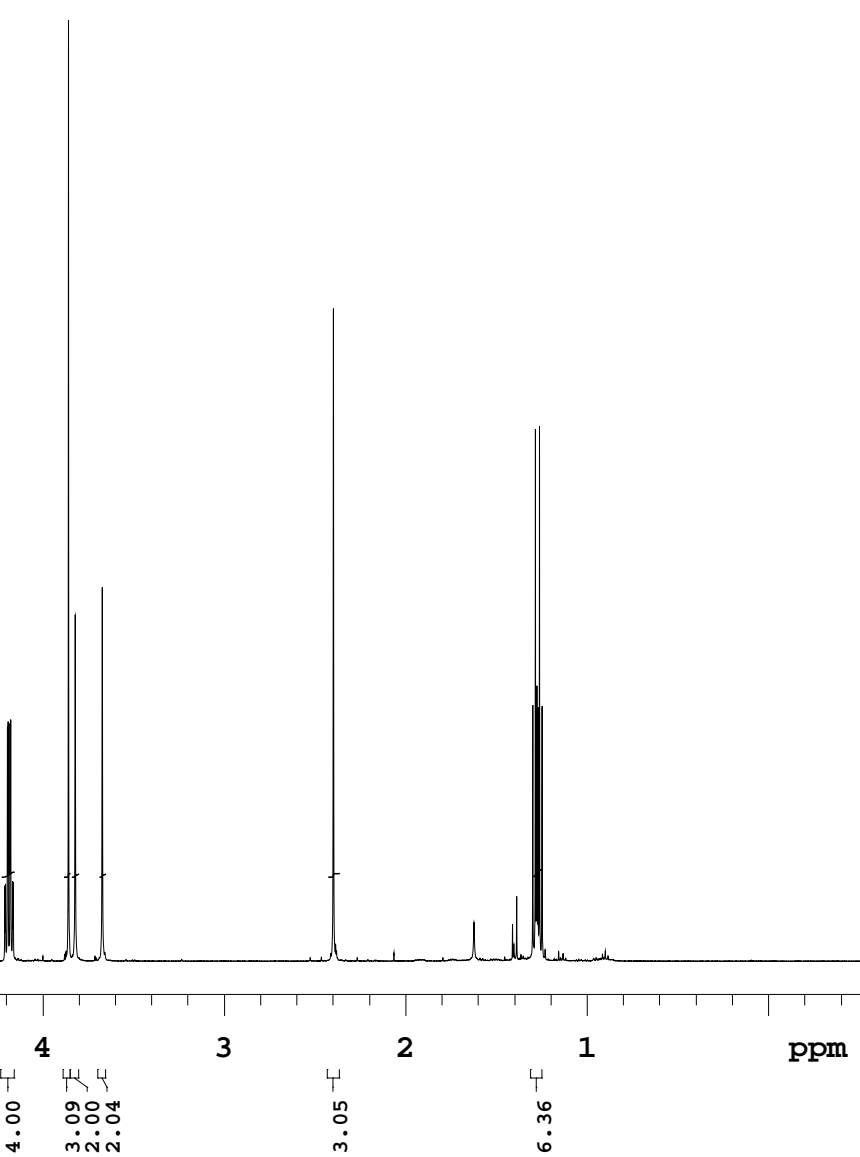

File: /mnt/d600/home13/rilnmr//mmrdata/DATA_FROM_NMRSERVICE/Patrick/2016.05/2016.05.24_u5_PM-07-193-F20-27_loc1_00.09_H1_1D 


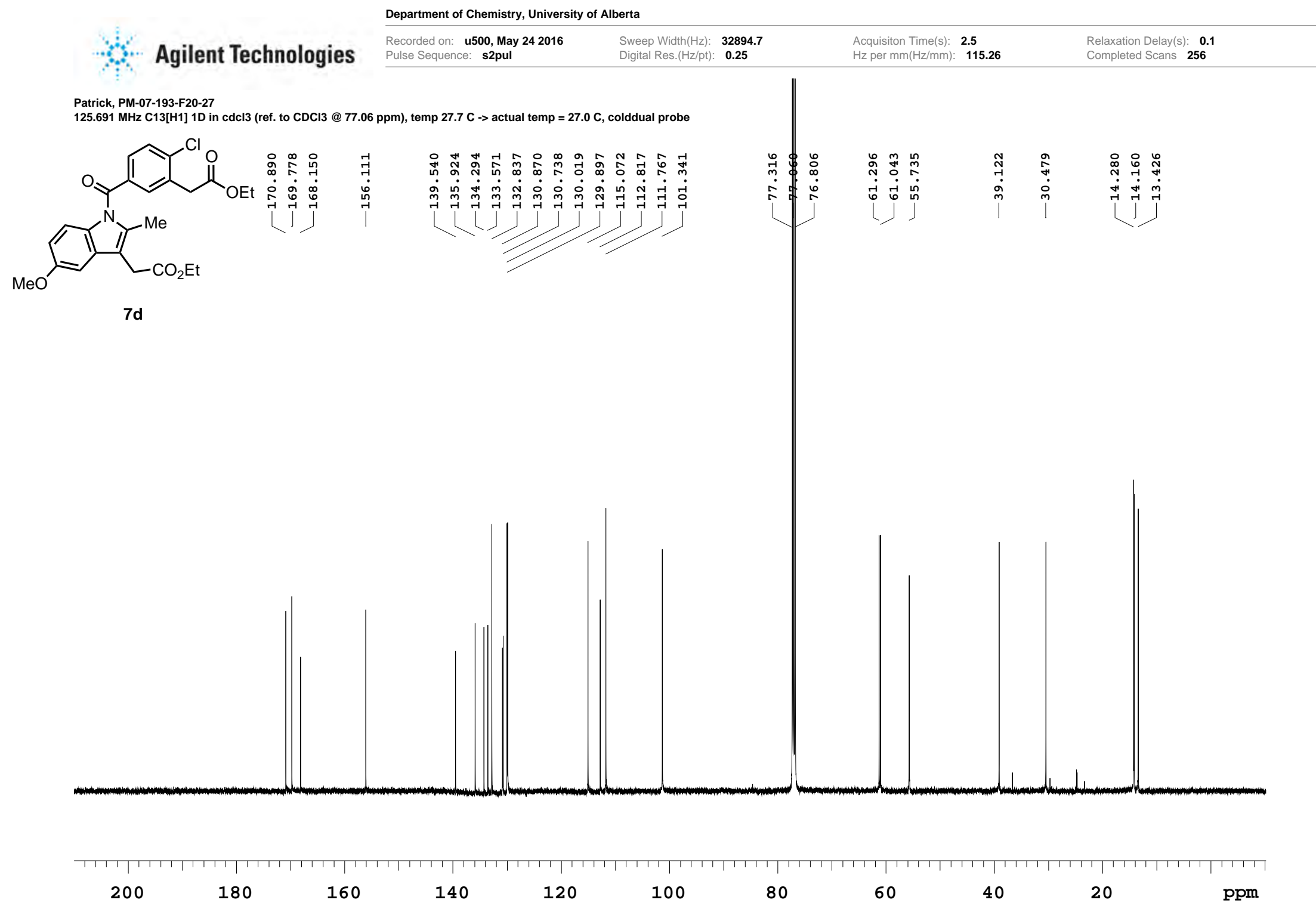

File: /mnt/d600/home13/rijnmr/hmmrdata/DATA_FROM_NMRSERVICE/Patrick/2016.05/2016.05.24.u5_PM-07-193-F20-27_loc1_00.11_C13_1D 